LA-14190

Approved for public release;

distribution is unlimited.

\title{
Data Analysis, Pre-Ignition Assessment, and Post-Ignition Modeling of the Large-Scale Annular Cookoff Experiments
}

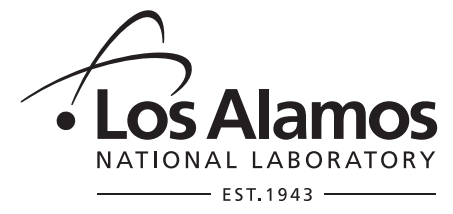

The World's Greatest Science Protecting America 
This report was funded by the US Department of Energy with additional financial support from the Surety Office.

Edited by Susan Rhyne, Butler Service Group, for Group IM-1.

Los Alamos National Laboratory, an affirmative action/ equal opportunity employer, is operated by the University of California for the United States Department of Energy under contract W-7405-ENG-36.

This report was prepared as an account of work sponsored by an agency of the United States Government. Neither the Regents of the University of California, the United States Government nor any agency thereof, nor any of their employees make any warranty, express or implied, or assume any legal liability or responsibility for the accuracy, completeness, or usefulness of any information, apparatus, product, or process disclosed, or represent that its use would not infringe privately owned rights. Reference herein to any specific commercial product, process, or service by trade name, trademark, manufacturer, or otherwise does not necessarily constitute or imply its endorsement, recommendation, or favoring by the Regents of the University of California, the United States Government, or any agency thereof. The views and opinions of authors expressed herein do not necessarily state or reflect those of the Regents of the University of California, the United States Government, or any agency thereof. Los Alamos National Laboratory strongly supports academic freedom and a researcher's right to publish; as an institution, however, the Laboratory does not endorse the viewpoint of a publication or guarantee its technical correctness. 
LA-14190

Issued: September 2005

Data Analysis, Pre-Ignition Assessment, and Post-Ignition Modeling of the

Large-Scale Annular Cookoff Experiment

Guillermo Terrones

Francisco J. Souto

Robert F. Shea

Michael W. Burkett

Edward S. Idar 


\section{Contents}

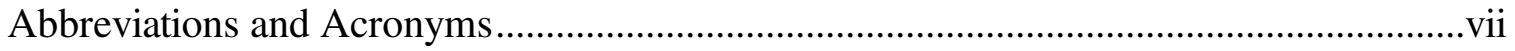

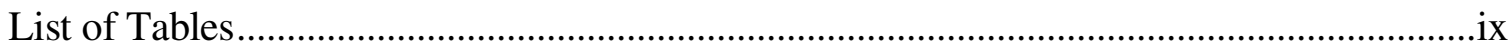

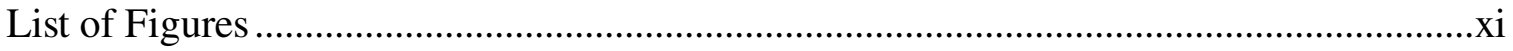

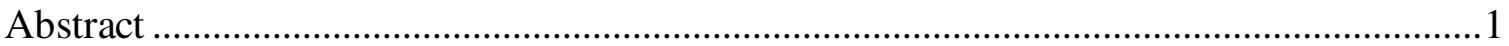

1 Introduction, Background, and Objective ............................................................. 3

2 Description of the Large-Scale Annular Cookoff Experiments ................................... 7

3 Analysis of the Large-Scale Annular Cookoff Experimental Results......................... 19

4 Pre-Ignition Hypotheses and Modeling of the Large-Scale Annular

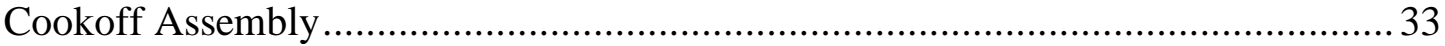

5 Baseline Simulations: Detonation of the Pristine Plastic-Bonded Explosive-9501 .... 57

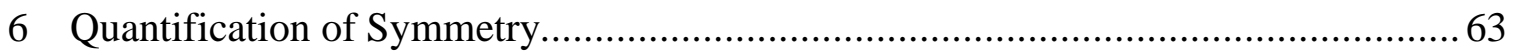

7 Modified Equation of State for Post-Ignition Numerical Simulations........................ 69

8 Inner-Liner Collapse: Numerical Simulation Results .............................................. 79

9 Outer-Liner and End-Cap Deformation: Numerical Simulation Results ................. 103

10 Interpretation of the Irregularities in the Field of View in the Collapsing

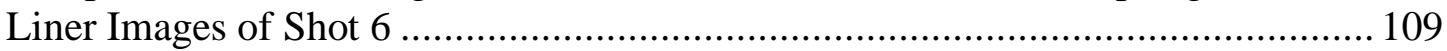

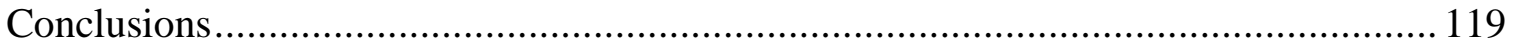

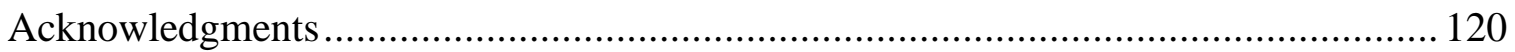

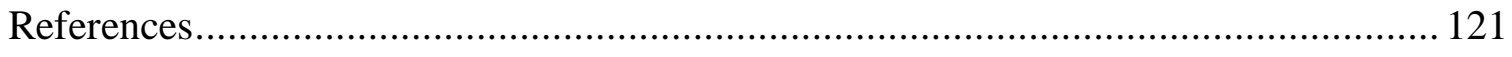

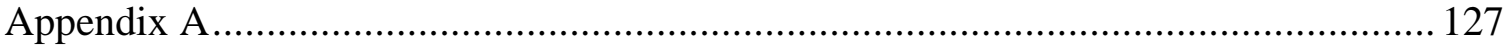

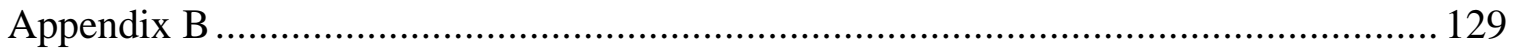

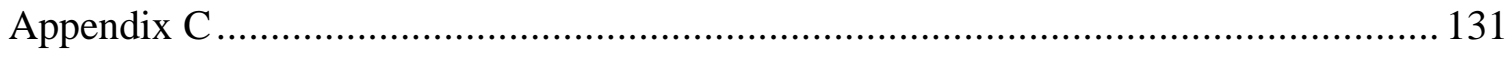




\section{Abbreviations and Acronyms}

BDNPA

BDNPA/F

BDNPF

CJ

C-PCS

D Division

D-5

DX

DX-2

DX-3

EOS

FMP

HE

HMX

JS

JWL

LANL

LLNL

LSAC

MTS

PBX

RW

SITI

SNL

TGA

VISAR

$\mathrm{X}-4$ bis(2,2-dinitropropyl)acetal

dual parts of bis(2,2-dinitropropyl)acetal and bis(2,2-

dinitropropyl)formal

bis(2,2-dinitropropyl)formal

Chapman-Jouguet

Physical Chemistry and Applied Spectroscopy group

Decision Applications

Nuclear Design and Risk Analysis group

Dynamic Experimentation Division

Materials Dynamics group

Hydrodynamics group

equation of state

fastest moving point

high explosive

high-melting explosive

Johnson spall

Jones-Wilkins-Lee

Los Alamos National Laboratory

Lawrence Livermore National Laboratory

large-scale annular cookoff

mechanical threshold stress

plastic-bonded explosive

resistance wire

Sandia instrumented thermal ignition

Sandia National Laboratories

thermogravimetric analysis

velocity interferometer system for any reflector

Primary Design and Assessment 


\section{List of Tables}

Table 2.1. Summary of the LSAC Assembly Tests ......................................................

Table 2.2. Dimensions of the LSAC Assemblies for Sizes A and B ..............................9

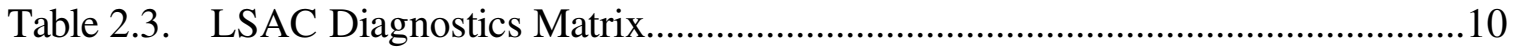

Table 2.4. Optic Fiber Peak Time for Shot 4 ................................................................. 16

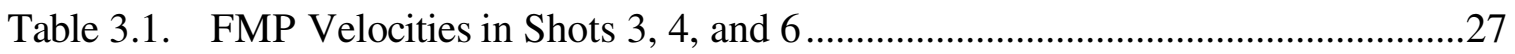

Table 3.2. Time of Arrival at Subsequent Sensors with Respect to the $0^{\circ}$ Sensor ..........29

Table 4.1. Mass Loss of Unconfined PBX-9501 (adapted from Smilowitz

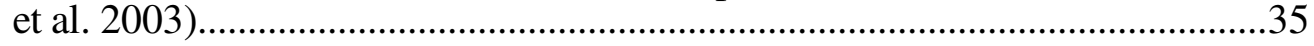

Table 4.2. Comparison between the Permeability Measured by the Sample Provider and the LANL Permeability Apparatus (measurements in $\mathrm{m}^{2}$ )..............................40

Table 7.1. JWL Parameters for PBX-9501 and an Artificially Degraded HE...................75

Table 9.1. Comparison between Simulations and Shot 6 Arrival Time...........................104

Table 9.2. Shot 6 Outer-Liner Reaction Speed from Accelerometer Data.......................105 


\section{List of Figures}

Fig. 2.1a. Model cutaway view of the size A copper LSAC assembly. ...........................

Fig. 2.1b. LSAC experimental assembly (brass casing) showing the heating tape and thermocouple transducers.

Fig. 2.2. LSAC assembly in the vacuum chamber. ......................................................

Fig. 2.3. Temperature evolution during the slow-heating phase of Shot 6. ...................11

Fig. 2.4. High-speed photographs during the fast phase of Shot 6 . .............................. 12

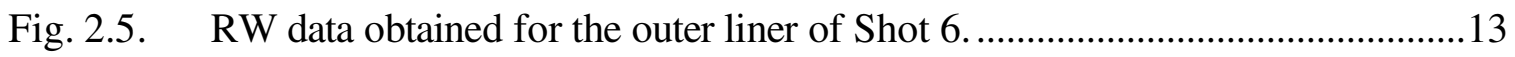

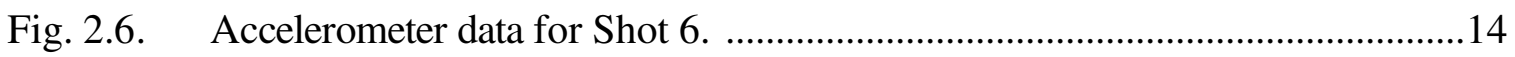

Fig. 2.7. VISAR data for an end cap in Shot 6. ........................................................ 14

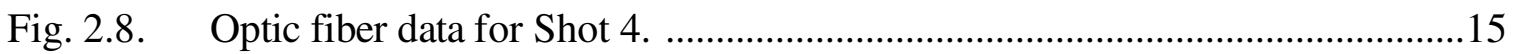

Fig. 2.9. Vacuum chamber pressure for Shot 6. ........................................................17

Fig. 3.1. Time to ignition for the LSAC shots. ..........................................................19

Fig. 3.2. Angular location (in degrees) of the peak temperature sensor vs. angular location (in degrees) of thermal runaway for five LSAC tests. .......................21

Fig. 3.3. Location of the FMP velocity vector at $30 \mu \mathrm{s}$ in Shot 6. ................................22

Fig. 3.4. Normalized radial displacement of the FMP, taken from the photographs of Shot 6. ............................................................................22

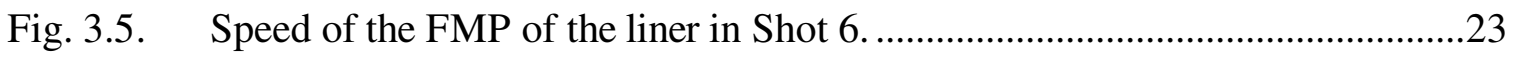

Fig. 3.6. Sequence of photographs taken of the collapsing inner liner in Shot 3. .........24

Fig. 3.7. Comparison between the estimated velocities of the FMPs in Shots 3

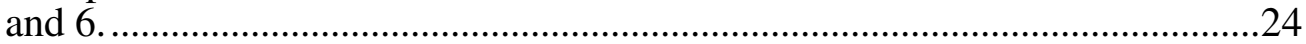

Fig. 3.8. Shot 8 after only a small portion of the HE ignited. .......................................25

Fig. 3.9. Inferred velocity of the "streak tip" at $165^{\circ}$ in Shot 3 .................................26

Fig. 3.10. Effect on the image after adjusting the brightness and the contrast in the frame at $50 \mathrm{~ms}$ in Shot 3.

Fig. 3.11. Location of the FMP velocity vectors at $70 \mu$ s in Shot 4 (grid thickness has been increased for clarity)

Fig. 3.12. Estimated velocities of the FMPs at $180^{\circ}$ and $345^{\circ}$ for Shot 4 ......................28

Fig. 3.13. Arc length as a function of time from the optic fiber data in Shot 4...............29

Fig. 3.14. Burn rate estimated from the optic fiber data in Shot 4 ...................................30

Fig. 3.15. Contours of normalized output from the optical fiber data in Shot 4 ................31

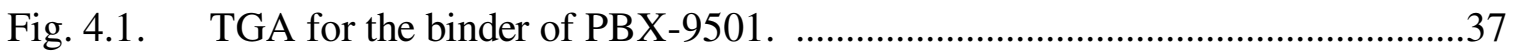

Fig. 4.2. Schematic depiction of a simple gas permeameter apparatus. ...........................38

Fig. 4.3. Normalized pressure as a function of time for a single-phase fluid flowing through an isothermal and chemically inert porous sample (normal Darcy conditions) 
Fig. 4.4. Schematic depiction of the permeameter used by Asay et al. (2004)...............41

Fig. 4.5. Pressure in the nitrogen tank, as a function of time.

Fig. 4.6. Accumulation of molten binder around the periphery of the low-pressure end of a cooked sample of PBX-9501 after nitrogen gas was fed through it from the other end (Run 12).

Fig. 4.7. Comparison of the pressure histories during different measurements of the permeability of cooked PBX-9501.

Fig. 4.8. Tank pressure as a function of sample temperature (showing that these quantities are not correlated).

Fig. 4.9. Flow of molten binder through the small annular region between the PBX9501 and an aluminum cylinder.

Fig. 4.10. Extrusion of a liquid through a porous medium by a gas.

Fig. 4.11. Dimensionless pressure versus time for a model in which liquid is flushed out of a porous sample by a gas.

Fig. 4.12. Dimensionless pressure versus time for a model during and after a hypothetical flushing of a liquid out of a porous sample (by a gas). .50

Fig. 4.13. Comparison of averaged thermocouple measurements for the inner, middle, and outer explosive regions for LSAC Shots 5 and 6 (copper liners).

Fig. 4.14. Time to ignition data within the cookoff temperature range.

Fig. 4.15. Comparison of averaged (azimuthally at a fixed radial location) thermocouple measurements for the inner, middle, and outer explosive regions for LSAC Shots 3 and 6.

Fig. 5.1. Base-case configurations simulated with pristine PBX-9501.........................57

Fig. 5.2. At $25 \mathrm{~ms}$, the velocity magnitude is shown for a one-point (left) and two-point (right) detonation of the LSAC assembly with pristine PBX-9501.

Fig. 5.3. At $30 \mathrm{~ms}$, the velocity magnitude is shown for one-point (left) and two-point (right) detonation of the LSAC assembly with pristine PBX-9501.

Fig. 5.4. Plastic strain-rate mapping on the interior of the assembly (color scale is in units of $\mathrm{ms}^{-1}$ ).

Fig. 6.1. Reference pin locations within the midplane of the assembly.........................63

Fig. 6.2. Shot 6 photographs of the inner liner collapse.

Fig. 6.3. Shot 6 photographs of the inner liner collapse, obscured by gaseous products.

Fig. 6.4. Digitized contours from Shot 6 framing sequence up to $70 \mathrm{~ms}$. .66

Fig. 6.5. Comparison of the relative time of arrival to pin locations versus angle curves between Shot 6 (red curves) and the one-point detonation numerical simulation with pristine PBX-9501 (blue curves)..........................................................66

Fig. 6.6. Time of arrival to pin locations versus angle curves for Shot 3. ...................67

Fig. 7.1. Expansion adiabats and their respective Rayleigh lines for the detonation products of PBX-9501 and degraded HE. 
Fig. 7.2. Expansion adiabat and Rayleigh line for detonation of PBX-9501 with the expansion adiabat for deflagration of PBX-9501. ..........................................76

Fig. 7.3. Cross section of a four-layered LSAC assembly HE model. ...........................77

Fig. 7.4. Inner-liner collapse sequence for the configuration depicted in Fig. 7.3..........78

Fig. 8.1. Pin curves for a simulated configuration (blue) initiated at a point near the outer liner with $\mathrm{D}=1 \mathrm{~km} / \mathrm{s}$ and $2 \%$ of $E_{P B X-9501}$. Shot 6 pin curves shown in red. 80

Fig. 8.2. Pin curves for a simulated configuration (blue) with $\mathrm{D}=1 \mathrm{~km} / \mathrm{s}$ and $50 \%$ of $E_{P B X-9501}$. The initiation point is located near the outer liner. Shot 6 pin curves are shown in red....

Fig. 8.3. Pin curves for a simulated configuration (blue) with $\mathrm{D}=1 \mathrm{~km} / \mathrm{s}$ and $100 \%$ of $E_{P B X-9501}$. The initiation point is located near the inner liner. Shot 6 pin curves are shown in red.

Fig. 8.4. Pin curves for a simulated configuration (blue) with $\mathrm{D}=1 \mathrm{~km} / \mathrm{s}$ and $100 \%$ of $E_{P B X-9501}$. The initiation point is located at the midpoint. Shot 6 pin curves are shown in red.

Fig. 8.5. Pin curves for a simulated configuration (blue) with $\mathrm{D}=1 \mathrm{~km} / \mathrm{s}$ and $100 \%$ of $E_{P B X-9501}$. The initiation point is located at the outer liner. Shot 6 pin curves are shown in red.

Fig. 8.6. Pin curves for a simulated configuration (blue) with $\mathrm{D}=4.4 \mathrm{~km} / \mathrm{s}$ and $46 \%$ of $E_{P B X-9501}$. The initiation point is located at the outer liner. Shot 6 pin curves are shown in red.

Fig. 8.7. Pin curves for a simulated configuration (blue) initiated at a point near the outer liner with $\mathrm{D}=1.4 \mathrm{~km} / \mathrm{s}$ and $100 \%$ of $E_{P B X-9501}$. Shot 6 pin curves are shown in red......

Fig. 8.8. Pin curves for a simulated configuration (blue) initiated at a point near the outer liner with $\mathrm{D}=2 \mathrm{~km} / \mathrm{s}$ and $100 \%$ of $E_{P B X-9501}$. Shot 6 pin curves are shown in red.

Fig. 8.9. Pin curves for a simulated configuration (blue) with $\mathrm{D}=1 \mathrm{~km} / \mathrm{s}$ and $100 \%$ of $E_{P B X-9501}$. Initiation points coincide with the pressure-front locus from a one-detonation point at the outer liner.

Fig. 8.10. Pin curves for a simulated configuration (blue) with $\mathrm{D}=1 \mathrm{~km} / \mathrm{s}$ and $100 \%$ of $E_{P B X-9501}$. Initiation points are on the locus of a small ellipsoid closer to the outer liner.

Fig. 8.11. Pin curves for a simulated configuration (blue) with $\mathrm{D}=1 \mathrm{~km} / \mathrm{s}$ and $100 \%$ of $E_{P B X-9501}$. Initiation points are on the locus of an ellipsoid that covers a sector larger than $90^{\circ}$.....

Fig. 8.12. Pin curves for a simulated configuration (blue) with $\mathrm{D}=2 \mathrm{~km} / \mathrm{s}$ and $100 \%$ of $E_{P B X-9501}$. Initiation points are on the locus of an ellipsoid that covers a sector larger than $90^{\circ}$. 
Fig. 8.13. Pin curves for a simulated configuration (blue) with $\mathrm{D}=1$ and $2 \mathrm{~km} / \mathrm{s}$ (inside and outside, respectively, of a crescent-shaped region that covers a sector larger than $270^{\circ}$ ) and $100 \%$ of $E_{P B X-9501}$. Initiation point is at midpoint.

Fig. 8.14. Pin curves for a simulated configuration (blue) with $\mathrm{D}=1$ and $2 \mathrm{~km} / \mathrm{s}$ (inside and outside, respectively, of a crescent-shaped region) and $100 \%$ of $E_{P B X-9501}$. Initiation points are around a stretched ellipsoid that covers a sector larger than $90^{\circ}$.

Fig. 8.15. Pin curves for a simulated configuration (blue). One-half of the HE has $\mathrm{D}=1 \mathrm{~km} / \mathrm{s}$, and the other half has $2 \mathrm{~km} / \mathrm{s}$; both halves have $100 \%$ of $E_{P B X-9501}$. Initiation point is at the outer liner.

Fig. 8.16. Pin curves for a simulated configuration (blue). One-half of the HE has $\mathrm{D}=1 \mathrm{~km} / \mathrm{s}$, and the other half has $9 \mathrm{~km} / \mathrm{s}$; both halves have $100 \%$ of $E_{P B X-9501}$. Initiation point is at the outer liner.

Fig. 8.17. Pin curves for a simulated configuration (blue). One-half of the HE has $\mathrm{D}=1 \mathrm{~km} / \mathrm{s}$, and the other half has $2 \mathrm{~km} / \mathrm{s}$; both halves have $100 \%$ of $E_{P B X-9501}$. Initiation points coincide with the pressure front locus from a one- initiation point located near the outer liner.

Fig. 8.18. Pin curves for a simulated configuration (blue). One-quarter of the HE has $\mathrm{D}=1 \mathrm{~km} / \mathrm{s}$, and the rest has $2 \mathrm{~km} / \mathrm{s}$; both halves have $100 \%$ of $E_{P B X-9501}$. Initiation point is located at the outer liner.

Fig. 8.19. Pin curves for a simulated configuration (blue). One-half of the HE has $\mathrm{D}=1 \mathrm{~km} / \mathrm{s}$, one-quarter has $2 \mathrm{~km} / \mathrm{s}$, and the rest has $9 \mathrm{~km} / \mathrm{s}$; all are with $100 \%$ of $E_{P B X-9501}$. Initiation point is located at the outer liner.

Fig. 8.20. Pin curves for a simulated configuration (blue). One-half of the HE has $\mathrm{D}=1 \mathrm{~km} / \mathrm{s}$, and the other half has $2 \mathrm{~km} / \mathrm{s}$; both halves have $100 \%$ of $E_{P B X-9501}$. Initiation points are on the locus of a small ellipsoid closer to the outer liner. .93

Fig. 8.21a. Azimuthal pressure distribution in the inner liner at three different times before complete collapse. One-half of the $\mathrm{HE}$ has $\mathrm{D}=1 \mathrm{~km} / \mathrm{s}$ and the other half has 2 $\mathrm{km} / \mathrm{s}$; both halves have $100 \%$ of $E_{P B X-9501}$. Initiation points are on the locus of a small ellipsoid close to the outer liner.

Fig. 8.21b. Azimuthal pressure distribution in the inner liner at three different times near complete collapse. One-half of the HE has $\mathrm{D}=1 \mathrm{~km} / \mathrm{s}$, and the other half has 2 $\mathrm{km} / \mathrm{s}$; both halves have $100 \%$ of $E_{P B X-9501}$. Initiation points are on the locus of a small ellipsoid close to the outer liner.

Fig. 8.22a. Azimuthal compression distribution in the inner liner at three different times before complete collapse. One-half of the HE has D = $1 \mathrm{~km} / \mathrm{s}$ and the other half has $2 \mathrm{~km} / \mathrm{s}$; both halves have $100 \%$ of $E_{P B X-9501}$. Initiation points are on the locus of a small ellipsoid close to the outer liner.

Fig. 8.22b. Azimuthal compression distribution in the inner liner at three different times near complete collapse. One-half of the HE has D = $1 \mathrm{~km} / \mathrm{s}$, and the other has $2 \mathrm{~km} / \mathrm{s}$; both halves have $100 \%$ of $E_{P B X-9501}$. Initiation points are on the locus of a small ellipsoid closer to the outer liner. 
Fig. 8.23a. Azimuthal pressure distribution in the inner liner at three different times before complete collapse. The entire HE is pristine PBX-9501 (D = $9 \mathrm{~km} / \mathrm{s})$, and the detonation point is located near the outer liner.

Fig. 8.23b. Azimuthal pressure distribution in the inner liner at three different times near complete collapse. The entire HE is pristine PBX-9501 (D = $9 \mathrm{~km} / \mathrm{s})$, and the detonation point is located near the outer liner.

Fig. 8.24a. Azimuthal compression distribution in the inner liner at three different times before complete collapse. The entire HE is pristine PBX-9501 ( $D=9 \mathrm{~km} / \mathrm{s})$, and the detonation point is located near the outer liner.

Fig. 8.24b. Azimuthal compression distribution in the inner liner at three different times near complete collapse. The entire HE is pristine PBX-9501 (D=9 km/s), and the detonation point is located near the outer liner.

Fig. 8.25. Comparison between the first four photographs of the inner-liner collapse contours in Shot 6 and those from our simulations.

Fig. 8.26a. Comparison between simulations (the detonation of pristine PBX-9501 and deflagration of cooked PBX-9501) and Shot 6 data for the inner-liner FMP.

Fig. 8.26b. Comparison between the velocities of the inner-liner FMP as interpolated from Shot 6 data and from simulations for the deflagration of PBX-9501....

Fig. 9.1. PAGOSA simulations for the pressure behavior at the accelerometer locations in Shot 6.

Fig. 9.2. Comparison between the RW data from Shot 6 and PAGOSA simulations for degraded PBX-9501.

Fig. 9.3. Plastic strain in the copper casing, calculated by PAGOSA 106

Fig. 9.4. Comparison between the VISAR data from Shot 6 and the PAGOSA calculations for degraded PBX-9501.

Fig. 10.1. Shot 6 framing camera image at $100 \mu \mathrm{s}$.

Fig. 10.2. Pressure as a function of time for a tracer particle located at $r=4.13 \mathrm{~cm}$, $\theta=90^{\circ}$, and $z=0$. One-half of the HE has D $=1 \mathrm{~km} / \mathrm{s}$, and the other half has $2 \mathrm{~km} / \mathrm{s}$; both halves have $100 \%$ of $E_{P B X-9501}$ (initiation points are on the locus of a small ellipsoid close to the outer liner).

Fig. 10.3. Pressure as a function of time for a tracer particle located at $r=4.13 \mathrm{~cm}, \theta=90^{\circ}, z=0$. The entire HE is pristine PBX-9501 $(\mathrm{D}=9 \mathrm{~km} / \mathrm{s})$, and the detonation point is located near the outer liner.

Fig. 10.4. Pressure and porosity profiles, as a function of time, for pristine PBX-9501 between 6 and $14 \mu$ s.

Fig. 10.5. Pressure and porosity profiles, as a function of time, for pristine PBX-9501 between 16 and $21 \mu \mathrm{s}$.

Fig. 10.6. Pressure and porosity profiles, as a function of time, for thermally damaged or degraded PBX-9501 between 40 and $180 \mu$ s. 
Fig. 10.7. Dimensionless most-unstable and cutoff wavelengths, as functions of the normalized pressure ................................................................................. 116 


\title{
Data Analysis, Pre-Ignition Assessment, and Post-Ignition Modeling of the Large-Scale Annular Cookoff Experiments
}

\author{
Guillermo Terrones \\ Francisco J. Souto \\ Robert F. Shea \\ Michael W. Burkett \\ Edward S. Idar \\ Group X-4 Primary Design and Assessment
}

\begin{abstract}
In order to understand the implications that cookoff of plastic-bonded explosive-9501 could have on safety assessments, we analyzed the available data from the large-scale annular cookoff (LSAC) assembly series of experiments. In addition, we examined recent data regarding hypotheses about pre-ignition that may be relevant to post-ignition behavior. Based on the post-ignition data from Shot 6, which had the most complete set of data, we developed an approximate equation of state (EOS) for the gaseous products of deflagration. Implementation of this EOS into the multimaterial hydrodynamics computer program PAGOSA yielded good agreement with the inner-liner collapse sequence for Shot 6 and with other data, such as velocity interferometer system for any reflector and resistance wires. A metric to establish the degree of symmetry based on the concept of time of arrival to pin locations was used to compare numerical simulations with experimental data. Several simulations were performed to elucidate the mode of ignition in the LSAC and to determine the possible compression levels that the metal assembly could have been subjected to during post-ignition.
\end{abstract}




\section{Introduction, Background, and Objective}

Slow, prolonged heating (cookoff) of PBX-9501, a plastic-bonded explosive (PBX), results in thermal runaway, a situation in which the thermally initiated chemical reaction initially propagates as a deflagration front but could potentially transition to a detonation. The cookoff program at Los Alamos National Laboratory (LANL) was established to understand the thermal, physical, and chemical mechanisms controlling the reaction violence occurring in PBX-9501 produced by cookoff.

In 1999, an experimental team was formed from two groups at LANL-the Materials Dynamics group (DX-2), in the Dynamic Experimentation Division (DX), and the Physical Chemistry and Applied Spectroscopy group (C-PCS), in the Chemistry Division. Between 1999 and 2002, the team performed a series of eight tests to investigate the effects of cookoff when PBX-9501 was heated in an axisymmetric, annular configuration. This configuration is known as the large-scale annular cookoff (LSAC) assembly and is described in Chapter 2.

Originally, the experimentalists wanted to explore the "worst-case cookoff scenario" to study reaction violence and inner-liner collapse in the LSAC assembly, which they thought would be accomplished by achieving a perfectly symmetric heating profile within the assembly. In practice, however, even small variations in thermal contact resistances throughout any axisymmetric assembly would preclude azimuthal symmetry of the temperature field. Not surprisingly, the thermocouple records indicated that radial and azimuthal symmetries were not realized during the tests. For example, the data showed unexpected temperature variations (in some cases as high as $30^{\circ} \mathrm{C}$ ) around the circumference of the assembly and nonmonotonic radial temperature distributions. The results of some of these tests can be found in three LANL technical reports: Asay et al. (1999) and Howe et al. (2000a and 2000b). The raw data are available on a CD (Asay et al. 2003). However, detailed accounts of all the tests have not yet been made available (at the time the present report was completed).

In this report, we describe our analysis of the LSAC and other related data and the numerical simulations performed by the Safety Assessment Team of X-4 in support of the cookoff program. Our analyses and computations were aimed at understanding the time scales and energy release rates during the early stages of the LSAC assembly deformation behavior after thermal runaway occurred. Much of our work was concentrated on Shot 6, which had the most complete set of data, including Imacon and Cordin high-speed photographs as well as data from thermocouples, accelerometers, strain-gages, resistance wires (RW), and the velocity interferometer system for any reflector (VISAR). We also conducted analyses and simulations for Shot 3 and Shot 4 but to a lesser extent because only a limited amount of data are available. Chapters 3 and 4 are devoted to data analysis from the LSAC assembly series and recent permeability data (Parker et al. 2003; Parker et al. 2004; Asay et al. 2004).

Cookoff of HE is characterized by two phases, namely, pre-ignition and post-ignition. The pre-ignition phase provides information about the thermodynamic state of the HE and the location and time at which thermal runaway occurs. However, the kinds of analyses and assessments performed at X-4 require knowledge of post-ignition because it 
provides information about the rate of deformation and compression of metal as a function of position and time. While pre-ignition answers the question of when and where thermal runaway will arise, post-ignition establishes the hydrodynamic behavior of ductile metal flow. Even though the location of ignition influences the topology of deformation of a metal assembly, its precise knowledge is not crucial from a numerical modeling perspective because the coordinates of this point (or region) can be easily varied in simulations. However, the peak pressure and the speed of propagation of the deflagration front are essential parameters in determining the dynamic deformation of metals. Therefore, pre-ignition behavior is of secondary importance when it comes to understanding metal motion.

To investigate the time-resolved deformation and dynamic behavior of the LSAC assembly, which was driven by the gases produced by the thermally initiated explosion of PBX-9501, a series of numerical simulations was carried out using the multimaterial hydrodynamics computer program PAGOSA (Kothe et al. 1993). This massively parallel ASC code is used to simulate the three-dimensional dynamic behavior of materials subjected to high-strain rates, like those produced by explosions. Thus, PAGOSA is adequately suited for performing numerical simulations of the post-ignition behavior in the LSAC assembly tests.

We approximated the effects of the deflagration products (resulting from cooked PBX-9501) on the dynamic behavior of the LSAC metal assembly by modifying the parameters in the equation of state (EOS) that describes the expansion isentrope of the detonation products for pristine PBX-9501. Although we used the Jones-Wilkins-Lee (JWL) EOS functional form (Fickett and Davis 2000) in our simulations, different parameter combinations were tried in an attempt to reproduce the experimental observations of the thermal explosions in the LSAC. We modeled the elastoplastic (workhardening) response of the copper casing material to dynamic loading using the modified Steinberg-Cochran-Guinan constitutive equations for the shear modulus and the yield strength (Steinberg et al. 1980; Steinberg and Lund 1989; Steinberg 1996). In our simulations, the explosions were started at relatively small, localized (initiation) regions using the programmed burn model, and a variety of different scenarios were analyzed.

Because the use of the JWL empirical model in several hydrocodes has repeatedly provided excellent agreement with experiments designed to investigate the effect of PBX-9501 detonation products on the dynamic deformation of various metals, we began our computational effort by using the JWL EOS in PAGOSA to simulate the LSAC assembly with pristine PBX-9501. These initial simulations, described in Chapter 5, formed our base case and were compared with the cookoff test data and with simulations using a modified JWL EOS for thermally damaged PBX-9501. A metric to quantify symmetry is introduced in Chapter 6. In our simulations, "cooked" PBX-9501 is defined as having the modifications that we made to the JWL parameters of pristine PBX in order to approximate the thermally damaged $\mathrm{PBX}-9501$ reaction behavior that was observed experimentally in Shot 6. Our approaches to modifying the parameter set of the JWL EOS in order to model deflagration with PAGOSA is documented in Chapter 7.

After the base case was established, we performed a series of simulations with different regions of simulated HE and different JWL parameters so we could identify a configuration that would capture the timing and topology of the inner-liner collapse data. We concentrated on analyzing the collapse of the inner liner in Shot 6, for which a 
sequence of five unambiguous images (taken from $30 \mu$ s to $70 \mu$ s) was recorded. Although four photographic images taken during Shot 3 could also be analyzed, their quality was poor compared with that of the images for Shot 6 . Therefore, because there were less data of lesser quality for Shot 3, there is more uncertainty in its analysis. We discuss our simulations and compare them with the data from Shot 6 in Chapter 8.

Accelerometer and RW data were collected for the outer liner in Shot 6, but no highspeed photographs were taken. Additionally, in Shot 6, there is only a short time interval $(30 \mu \mathrm{s})$ during which VISAR data were recorded at one of the end caps. We used this limited data to interpret the behavior of the outer liner and the end cap and compared it with our simulations. We discuss these results in Chapter 9.

Finally, we tried to determine if some of the dark areas in several photographs of the inner-liner collapse could be metal, as originally reported (Howe et al. 2000b). Some experimentalists have suggested that the dark areas are metal that originated from either spall fracture or a hydrodynamic instability at the inner liner. This issue is addressed in Chapter 10, where we present (1) simulations with PAGOSA to compute the stress field of the inner liner, (2) MESA-2D simulations using the mechanical threshold stress (MTS) strength model (Follansbee and Kochs 1988) and the Johnson spall (JS) model (Johnson 1981; Johnson and Addessio 1988), and (3) an analysis to determine whether or not the pressures produced during cookoff would be high enough for a Rayleigh-Taylor instability to develop at the inner liner. 


\section{Description of the Large-Scale Annular Cookoff Experiments}

As part of the LSAC program, a team from DX-2 and C-PCS conducted a series of experiments to investigate the behavior of the HMX-based explosive PBX-9501 when it is slowly heated (Asay et al. 1999; Howe et al. 2000a). The experiments were done to provide information about the time and location of the ignition region, as well as the violence of the subsequent thermal explosion in the LSAC assembly.

The LSAC assembly consists of two concentric metal cylinders encasing the energetic material PBX-9501. Two configurations, referred to as $A$ and $B$, with different assembly dimensions and explosive-charge masses were instrumented and tested. Figure 2.1a shows a cross section of the copper $A$ assembly for Shot 6 . In this computational model, copper is shown in orange and the HE in yellow. The thicknesses of the outer and inner cylindrical liners are $1.27 \mathrm{~cm}(0.5 \mathrm{in}$.) and $0.64 \mathrm{~cm}(0.25 \mathrm{in}$.$) ,$ respectively. The HE was confined at both ends of the cylinders by end caps. Gaps between the HE and the end caps allowed for thermal expansion and volumetric increase caused by the phase transition of the HMX crystals from the $\beta$ to the $\delta$ allotrope. Figure $2.1 \mathrm{~b}$ shows a typical LSAC assembly with the heating tape surrounding the outer liner between the end caps, as well as a series of thermocouples and other transducers.

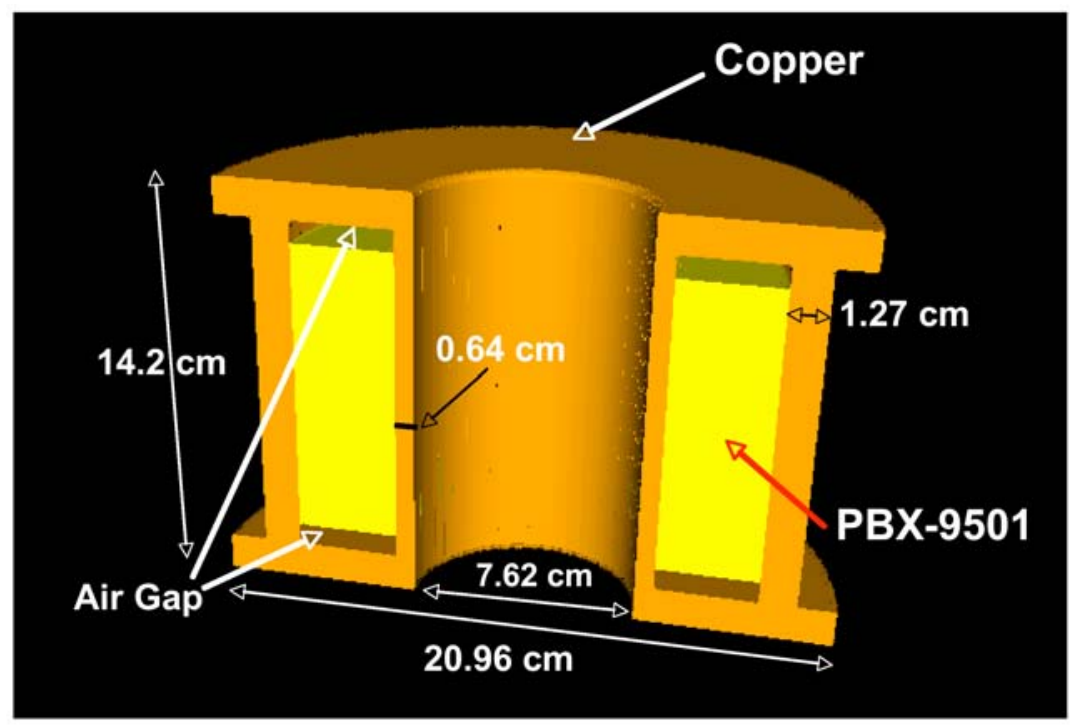

Fig. 2.1a. Model cutaway view of the size A copper LSAC assembly. 


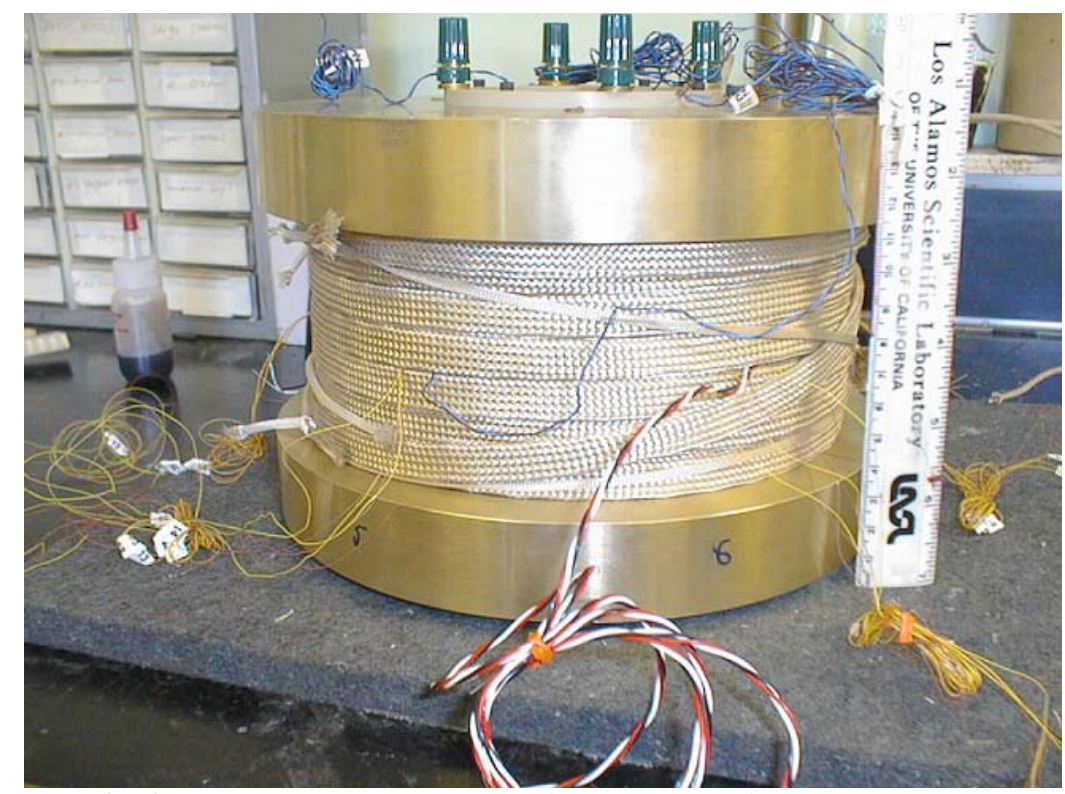

Fig. 2.1b. LSAC experimental assembly (brass casing) showing the heating tape and thermocouple transducers. (Photo provided by DX-2.)

Table 2.1 summarizes the eight experiments conducted from 1999 to 2002 and Table 2.2 lists the dimensions for the $A$ and $B$ LSAC configurations.

Table 2.1. Summary of the LSAC Assembly Tests

\begin{tabular}{|c|l|c|c|}
\hline Shot & Date & Size & Casing Material \\
\hline 1 & 29 April 1999 & A & Copper \\
\hline 2 & 16 June 1999 & A & Brass \\
\hline 3 & 10 September 1999 & A & Brass \\
\hline 4 & 10 February 2000 & A & Brass \\
\hline 5 & 9 October 2002 & A & Copper \\
\hline 6 & 18 October 2002 & A & Copper \\
\hline 7 & 2 November 2002 & B & Copper \\
\hline 8 & 4 December 2002 & B & Copper \\
\hline
\end{tabular}


Table 2.2. Dimensions of the LSAC Assemblies for Sizes A and B

\begin{tabular}{|l|c|c|}
\hline Dimension & Size A (cm) & Size B (cm) \\
\hline PBX-9501 Length & 10.16 & 20.32 \\
\hline PBX-9501 Inner Radius & 4.45 & 10.16 \\
\hline PBX-9501 Outer Radius & 7.94 & 13.65 \\
\hline PBX-9501 Thickness & 3.49 & 3.49 \\
\hline Outer Liner Thickness & 1.27 & 1.27 \\
\hline Inner Liner Thickness & 0.64 & 0.64 \\
\hline \multicolumn{2}{|c|}{ (Data provided by DX-2) }
\end{tabular}

As shown in Fig. 2.2, the entire LSAC assembly was suspended inside a vacuum chamber and was heated at about $2^{\circ} \mathrm{C}$ per minute to approximately $150^{\circ} \mathrm{C}$ with heating tapes wrapped around the walls of the outer cylindrical liner. This temperature was maintained for more than an hour to achieve a relatively uniform temperature distribution in the assembly, as determined by the thermocouple data. Heating the PBX-9501 was then continued to a temperature of about $180^{\circ} \mathrm{C}$ until ignition or thermal runaway. The various types of diagnostic instruments installed and the data obtained for each of the eight LSAC shots are listed in Table 2.3. In addition to those diagnostics, the heat flux to the assembly and the pressure in the vacuum chamber were also recorded for some tests. Notice that the most complete set of data was gathered for Shot 6, where all but the x-ray data were obtained.

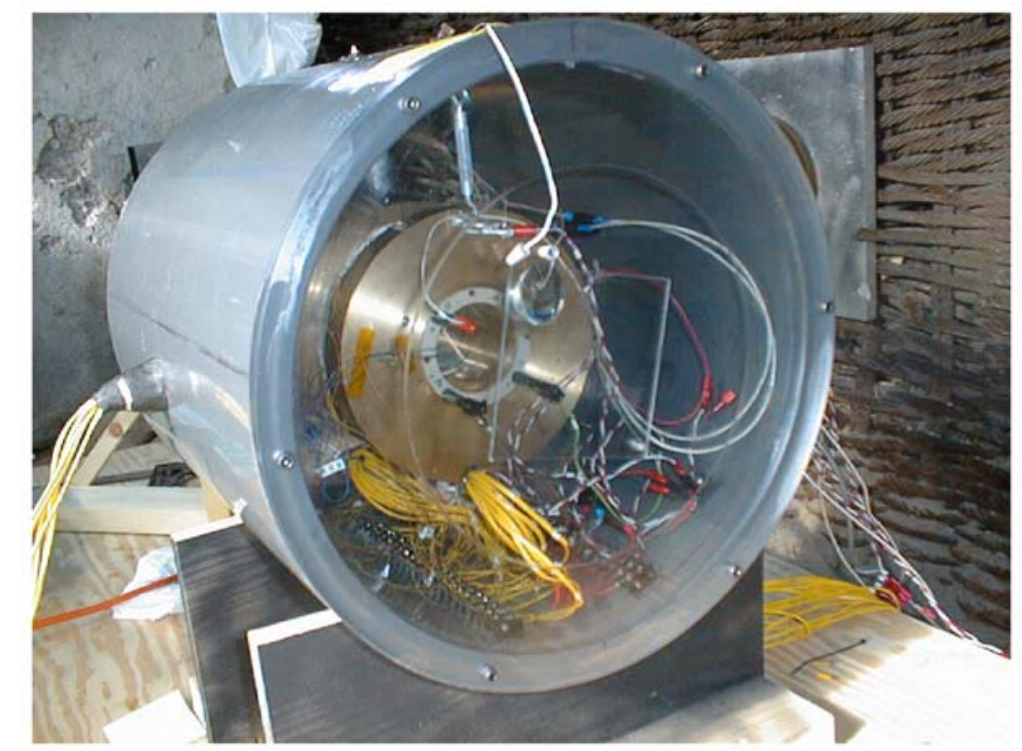

Fig. 2.2. LSAC assembly in the vacuum chamber. (Photo provided by $D X-2$. 
Table 2.3. LSAC Diagnostics Matrix

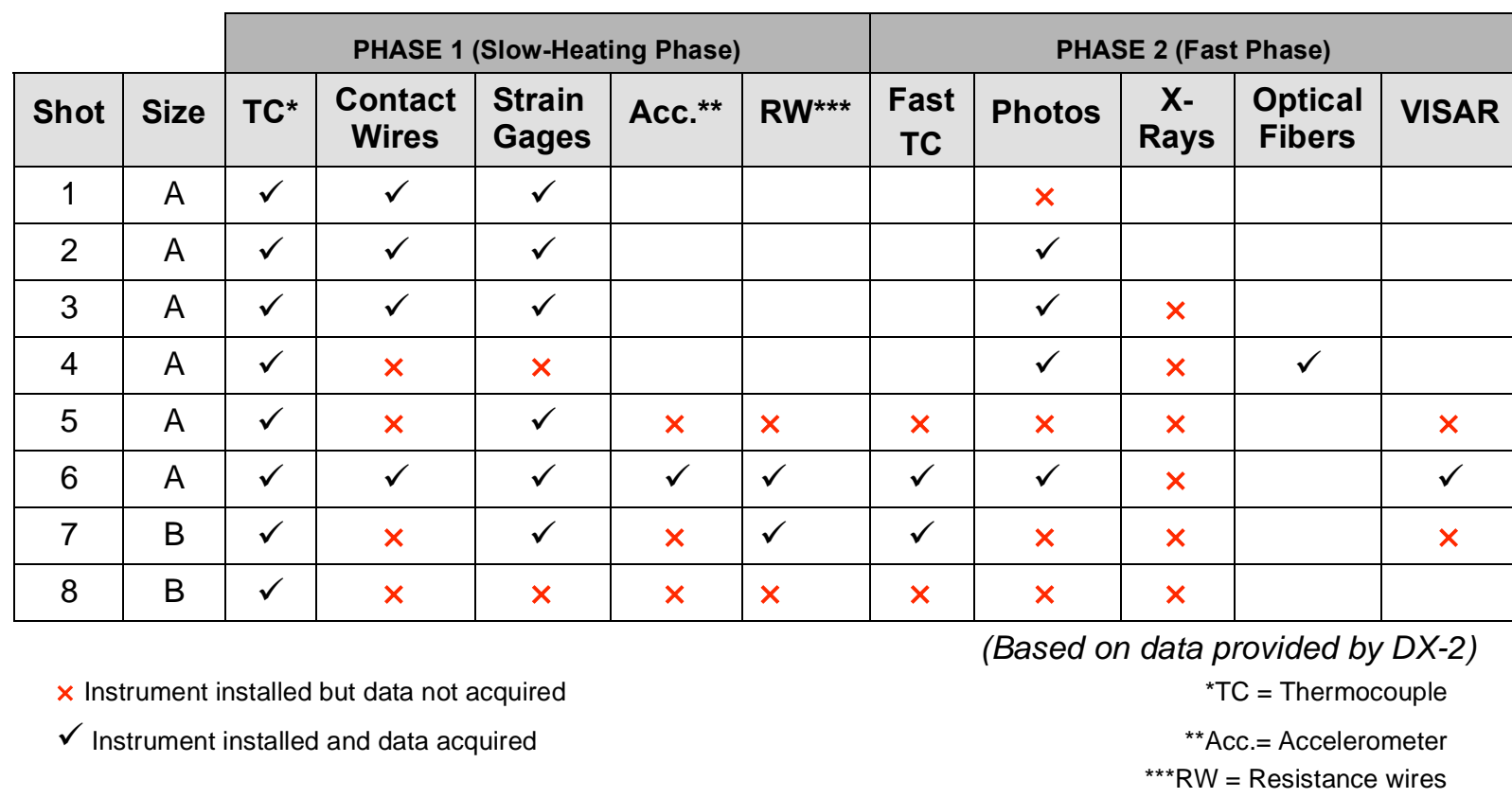

The LSAC experiments are characterized by two phases of data acquisition. The first phase records the slow heating up to the point of thermal runaway and encompasses the thermocouple and strain-gage data as well as, if applicable, the accelerometer and resistance wire data. The second phase begins when one of four contact wires, each located $90^{\circ}$ apart inside the inner cavity of the assembly, makes contact with the inner liner of the casing, which is initially about $1 \mathrm{~mm}$ away (no tolerance for this distance was recorded). This contact between the inner liner and the wire triggers the high-speed photography and, if applicable, $x$-ray, optical-fiber, and VISAR instrumentation.

In general, the diagnostics in the second - or fast—phase failed if the contact wires failed, as indicated in Table 2.3. The exceptions were in Shots 1 and 4. In Shot 1, the decomposition gases generated during the slow-heating process clouded the vacuumchamber window and, therefore, no useful photos were obtained. In Shot 4, apparently a spurious contact-wire signal triggered the high-speed photography and the optical fiber data in such a way that useful data were recorded.

With the exception of Shot 8, which partially ignited, all the LSAC tests resulted in violent explosions. In other words, in all cases except Shot 8 , the assembly was reported to be "completely" destroyed. 


\subsection{Some Large-Scale Annular Cookoff Experimental Results}

\subsubsection{Temperature Behavior}

Figure 2.3 shows the average temperatures measured on Shot 6 by thermocouples for the outer liner, end caps, inner liner, and PBX-9501, which are typical of other shots. In this shot, 18 thermocouples were uniformly distributed along the midplane of the HE. Notice that the $\beta$ to $\delta$ phase transition, starting at approximately $172^{\circ} \mathrm{C}$, is an endothermic reaction and is characterized by the temperature decrease observed to start at 2.9 hours and end at 3.5 hours. In this case, the explosive ignition, or thermal runaway, occurs after approximately 5.7 hours from the start of the experiment at an average temperature of $191^{\circ} \mathrm{C}$ within the $\mathrm{HE}$.

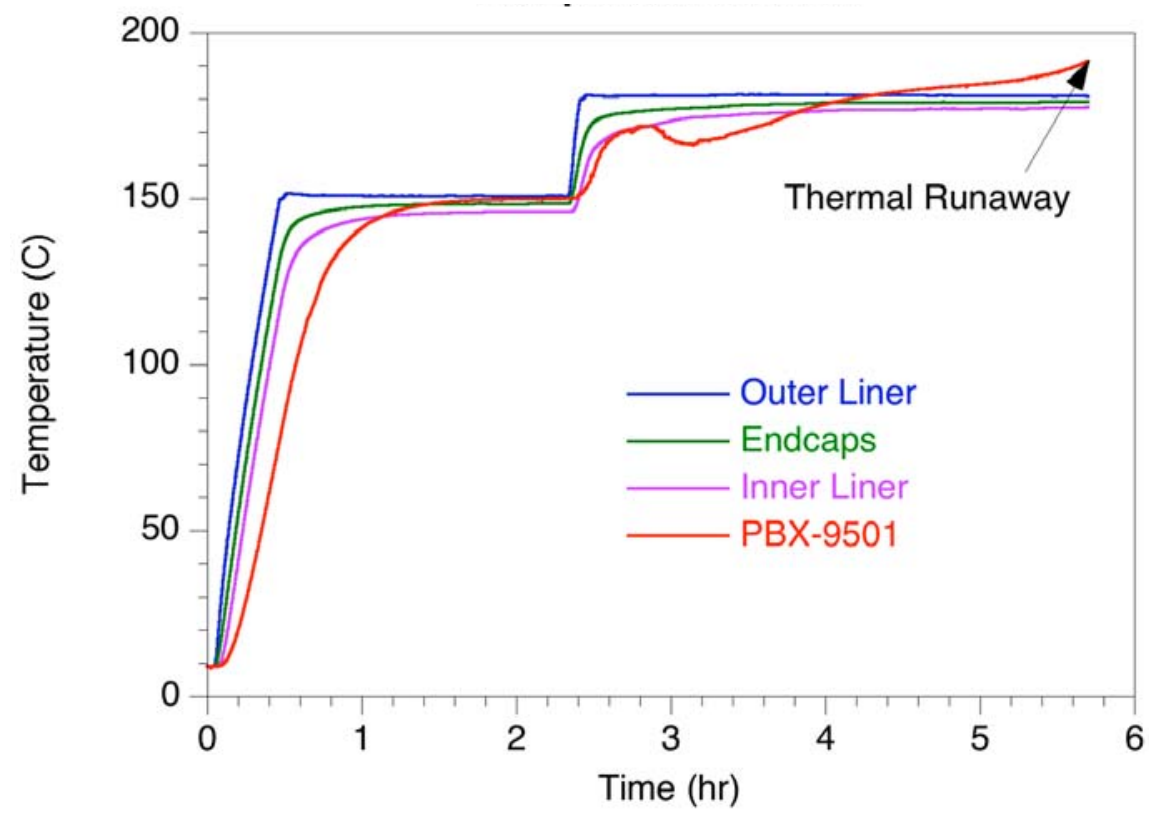

Fig. 2.3. Temperature evolution during the slow-heating phase of Shot 6. (Based on data provided by $D X-2)$

\subsubsection{High-Speed Photography}

Figure 2.4 shows the sequence of high-speed photographs recorded during Shot 6 taken over the first $100 \mu \mathrm{s}$ of the fast phase of the experiment. In this particular test, two types of high-speed framing cameras were used: an Imacon brand camera that was programmed to take photographs at $30,50,70$, and $90 \mu$ s and a Cordin 220 brand camera that was programmed to take photographs at 40,60,80, and $100 \mu$ s (both sequences of photographs to be taken with respect to the start of the fast phase). Each one of the photographs in Fig. 2.4 shows the inner-liner contour of the LSAC assembly overlaid on top of the static photograph, that is, a photograph taken before the fast phase began. 


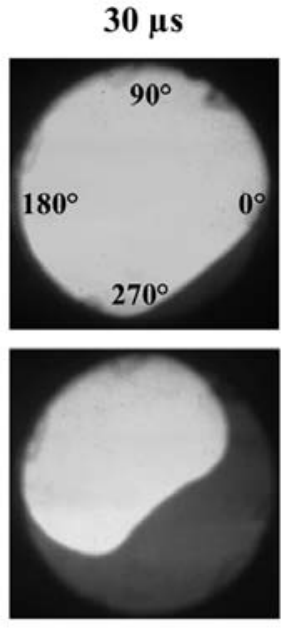

$70 \mu \mathrm{s}$
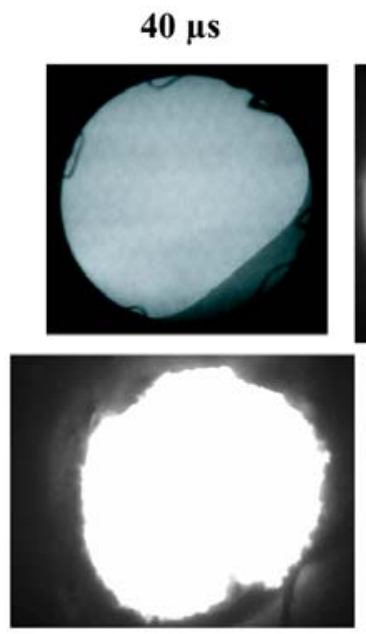

$80 \mu \mathrm{s}$
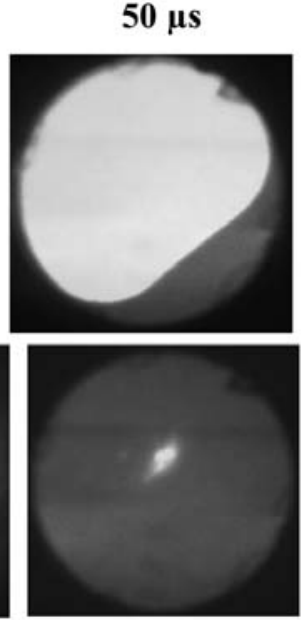

$90 \mu \mathrm{s}$
$60 \mu \mathrm{s}$
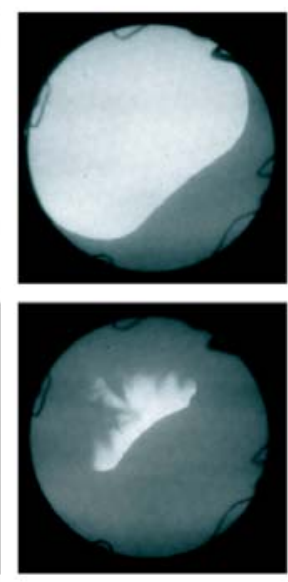

$100 \mu \mathrm{s}$

Fig. 2.4. High-speed photographs during the fast phase of Shot 6. (Data provided by $D X-2$.)

These photographs provide valuable information including the angular location of the initial reaction, the inner-wall velocity, and the degree of symmetry of the collapse. For example, the inner-liner wall starts moving at an angular location of approximately $315^{\circ}$. Some other events of interest may also be seen in the high-speed photographs taken during the experiment, for example, the features observed at $90 \mu \mathrm{s}$ and $100 \mu \mathrm{s}$. We believe these features are gases released by confinement failure before the assembly was completely destroyed and that these gases are the reaction products of the deflagration of PBX-9501 after thermal runaway. We emphasize that the framing camera images in Fig. 2.4 are shown in the order in which they were experimentally recorded. There appears to be some evidence that the images at 80 and $100 \mu \mathrm{m}$ are swapped because of an inadvertent swapping of channels in the Cordin 220 camera used in Shot 6 (see Appendix A for further details).

\subsubsection{Resistance Wire Data}

The resistance wire data can be used to deduce the behavior of the outer liner during the transient. Figure 2.5 shows the RW data obtained during the post-ignition phase of Shot 6 . RW 1 was located near one end cap of the assembly, whereas RW 2 was located around the middle region of the outer liner. Notice that in Fig. 2.5, the outer liner (at RW 2) begins to expand approximately $65 \mu \mathrm{s}$ after initiation of the second phase of the experiment and reaches a maximum expansion of about $35 \%$ at $150 \mu \mathrm{s}$. Because of the higher strain measured by RW 2, it appears that the ignition point in this case was located close to the middle region of the assembly. There is also a sharp decrease in the strain between $85 \mu \mathrm{s}$ and $90 \mu \mathrm{s}$, which might indicate confinement failure. 


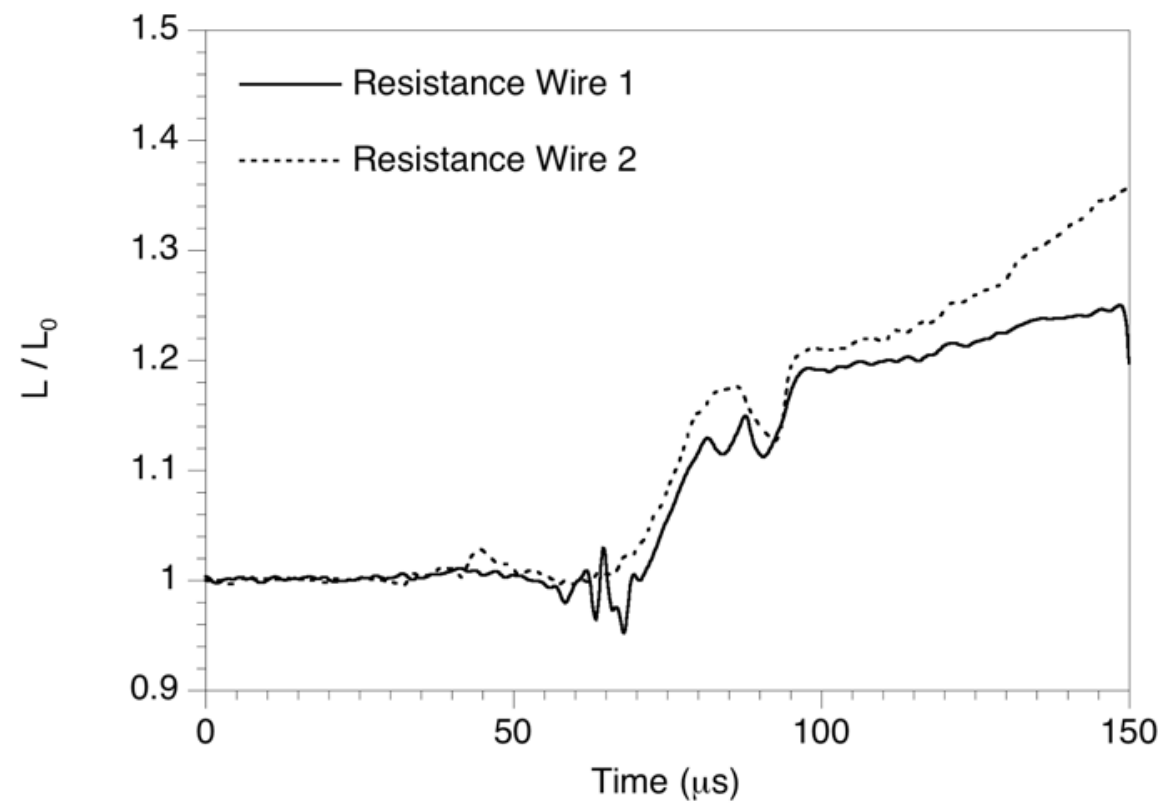

Fig. 2.5. RW data obtained for the outer liner of Shot 6. (Data provided by DX-2.)

\subsubsection{Accelerometer Data}

Data taken with accelerometers during the LSAC experiments can be used to detect the onset of motion in the outer liner. In Shot 6, three accelerometers were installed on the outer liner of the assembly. Accelerometer 1 was located at the middle region of the assembly, approximately $7 \mathrm{~cm}$ from one end and at $150^{\circ}$, with respect to the photographs in Fig. 2.4. Accelerometer 2 was located at approximately $8.5 \mathrm{~cm}$ from the same end and at $210^{\circ}$, and accelerometer 3 was located $5 \mathrm{~cm}$ from the same end and at $75^{\circ}$. For Shot 6 , the accelerometer data in Fig. 2.6 show that the outer liner started to move at approximately $45 \mu \mathrm{s}$ (during the second phase of the experiment). According to the photographs in Fig. 2.4 from Shot 6, the reaction occurs at an angular location of approximately $315^{\circ}$. The accelerometer data in Fig. 2.6 indicate that the signal from the thermal ignition traveled through the outer liner and first reached accelerometer 2, located at an angular location of $210^{\circ}$, then accelerometer 3, located at an angular location of $75^{\circ}$, and finally reached accelerometer 1 , located at an angular location of $150^{\circ}$. This sequence is consistent with an ignition located at about $315^{\circ}$ and propagating around the outer liner. 


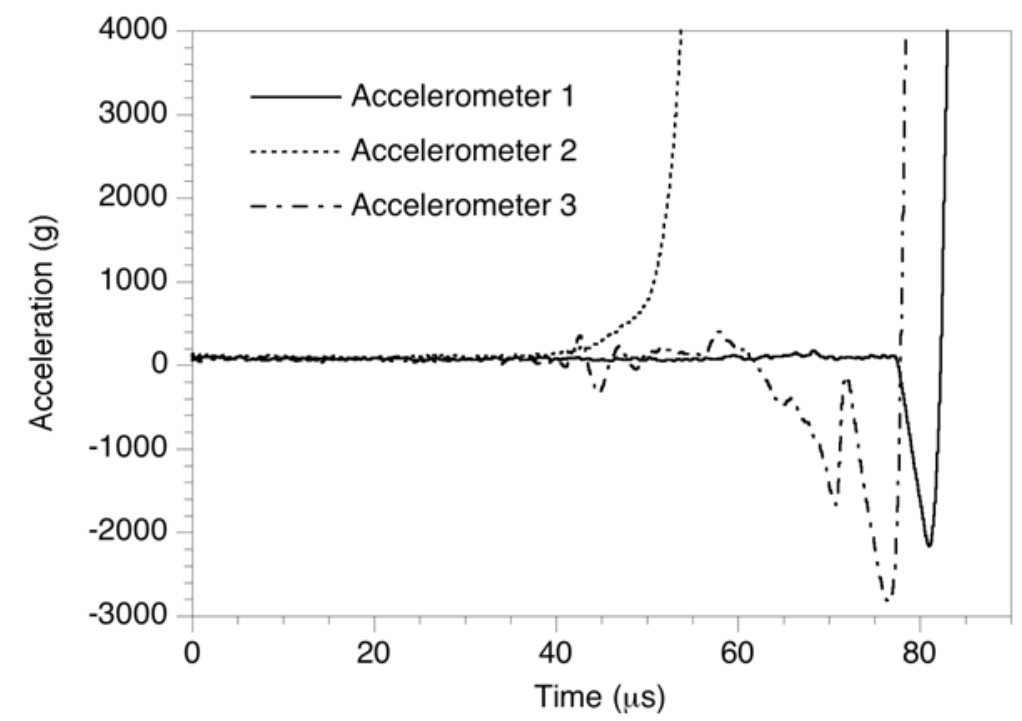

Fig. 2.6. Accelerometer data for Shot 6. (Data provided by $D X-2$.

\subsubsection{Velocity Interferometer System for Any Reflector Data}

The VISAR data provide an estimate of the wall velocity during the LSAC experiments. A VISAR was used in Shot 6 pointing toward one end cap of the assembly at an angular location of approximately $330^{\circ}$, midway between the outer and inner edges of the end cap. Figure 2.7 shows that the end cap of this assembly started moving at approximately $5 \mu$ s into the second phase of the experiment, and the velocity exceeded $100 \mathrm{~m} / \mathrm{s}$ at $30 \mu \mathrm{s}$. In addition, the VISAR record indicates that a shock wave did not arrive at the end cap during the initial $30 \mu$ s of acceleration.

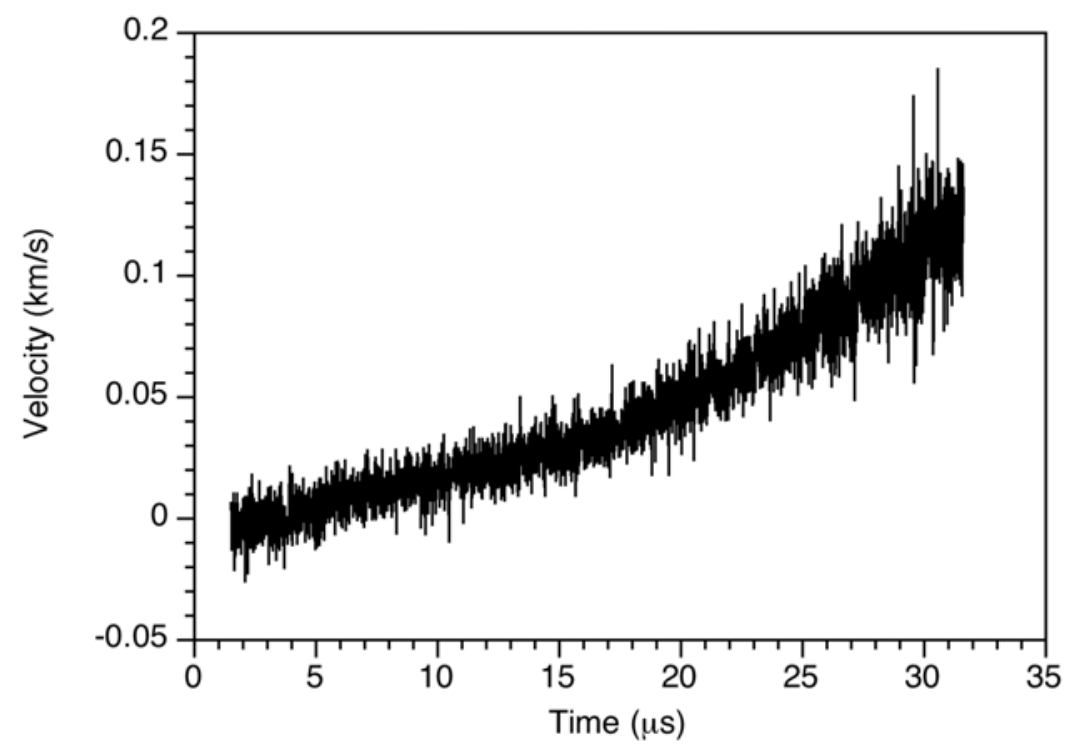

Fig. 2.7. VISAR data for an end cap in Shot 6. (Data provided by DX-3.) 


\subsubsection{Optical Fiber Data}

In order to determine the ignition location, Shot 4 was instrumented with 12 optical fibers (in the midplane), equally spaced along the angular position (from $0^{\circ}$ to $330^{\circ}$ ) at the outer edge of the explosive material. These optical fiber data can also be used to deduce the propagation of the burn within the heated PBX-9501 explosive material. The raw data recorded by the optical fibers in the first quadrant of the explosive during Shot 4 , that is, the optical fibers at $0^{\circ}$ through $90^{\circ}$, are shown in Fig. 2.8. A useful piece of information from the optical-fiber data is the time at which the signal peaks, which can be interpreted as the time of arrival of light, presumably from the burn reaction, to the specific location of the optical fiber. The data in Fig. 2.8 suggest that light from an ignition region first arrived at the optical fiber sensor located at $0^{\circ}$ then propagated sequentially to the sensors at $30^{\circ}, 60^{\circ}$, and $90^{\circ}$. The time of the optical fiber peak output for each sensor, from data similar to those shown in Fig. 2.9, is presented in Table 2.4. Notice that some optical fiber sensors detect the arrival of two or more signals; therefore, the times in Table 2.4 correspond to the highest peak for each sensor. As indicated in Table 2.4, there appear to have been two ignition regions in Shot 4: one at an angular location of approximately $180^{\circ}$ and the other at approximately $0^{\circ}$. As will be discussed in Chapter 3, this conclusion has been confirmed by the analysis of the high-speed photographs for Shot 4 . Shot 4 was the only instance in the entire series of tests where two ignition regions were recorded in the framing camera images.

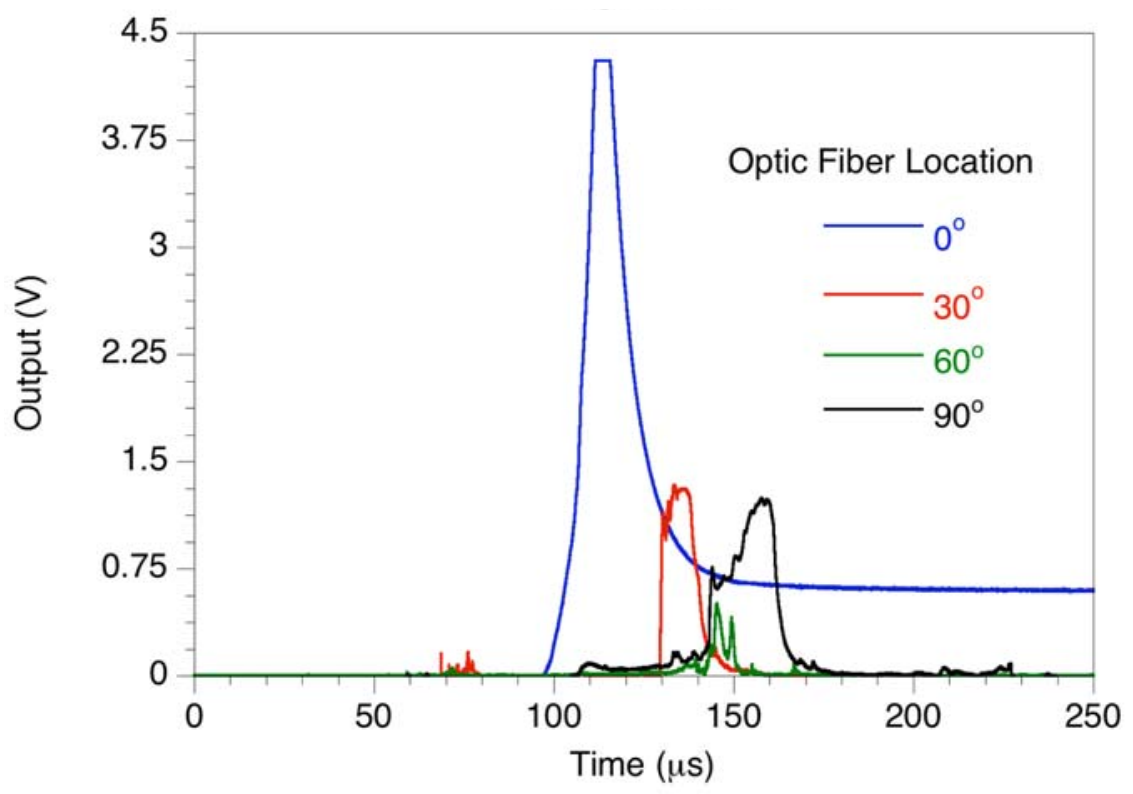

Fig. 2.8. Optical fiber data for Shot 4. (Data provided by $D X-2$.) 
Table 2.4. Optical Fiber Peak Time for Shot 4 (Based on data provided by DX-2)

\begin{tabular}{|c|c|}
\hline $\begin{array}{c}\text { Optical } \\
\text { Fiber } \\
\text { Location }\end{array}$ & $\begin{array}{c}\text { Output- } \\
\text { Peak } \\
\text { Time }(\boldsymbol{\mu s})\end{array}$ \\
\hline $0^{\circ}$ & 111 \\
\hline $30^{\circ}$ & 133 \\
\hline $60^{\circ}$ & 145 \\
\hline $90^{\circ}$ & 158 \\
\hline $120^{\circ}$ & 164 \\
\hline $150^{\circ}$ & 152 \\
\hline $180^{\circ}$ & 74 \\
\hline $210^{\circ}$ & 159 \\
\hline $240^{\circ}$ & 161 \\
\hline $270^{\circ}$ & 153 \\
\hline $300^{\circ}$ & 166 \\
\hline $330^{\circ}$ & 135 \\
\hline
\end{tabular}

\subsubsection{Vacuum Chamber Pressure}

The behavior of the pressure in the vacuum chamber, in which the LSAC assembly was suspended, was also measured during some of the experiments. Figure 2.9 shows the pressure history in the vacuum chamber during Shot 6. In Shot 6, approximately 4.2 hours into the slow-heating phase of the experiment, the pressure in the vacuum chamber begins to increase steadily, while the vacuum pump was operating, up to thermal runaway at about 5.6 hours (notice that the signal is lost before this time is reached). This pressure increase might be caused by the leakage of gases generated from the decomposition of PBX-9501 during the heating process, which would suggest that the LSAC design was not hermetic. After 4 hours, heat is being generated within the $\mathrm{HE}$ faster than it can be dissipated by conduction, resulting in a mean temperature greater than $180^{\circ} \mathrm{C}$ but less than $191^{\circ} \mathrm{C}$ (Fig. 2.3). Within this temperature range, the degree of decomposition for unconfined small size samples of PBX-9501 at 1 atmosphere of pressure is about 5\% (Smilowitz et al. 2003). 


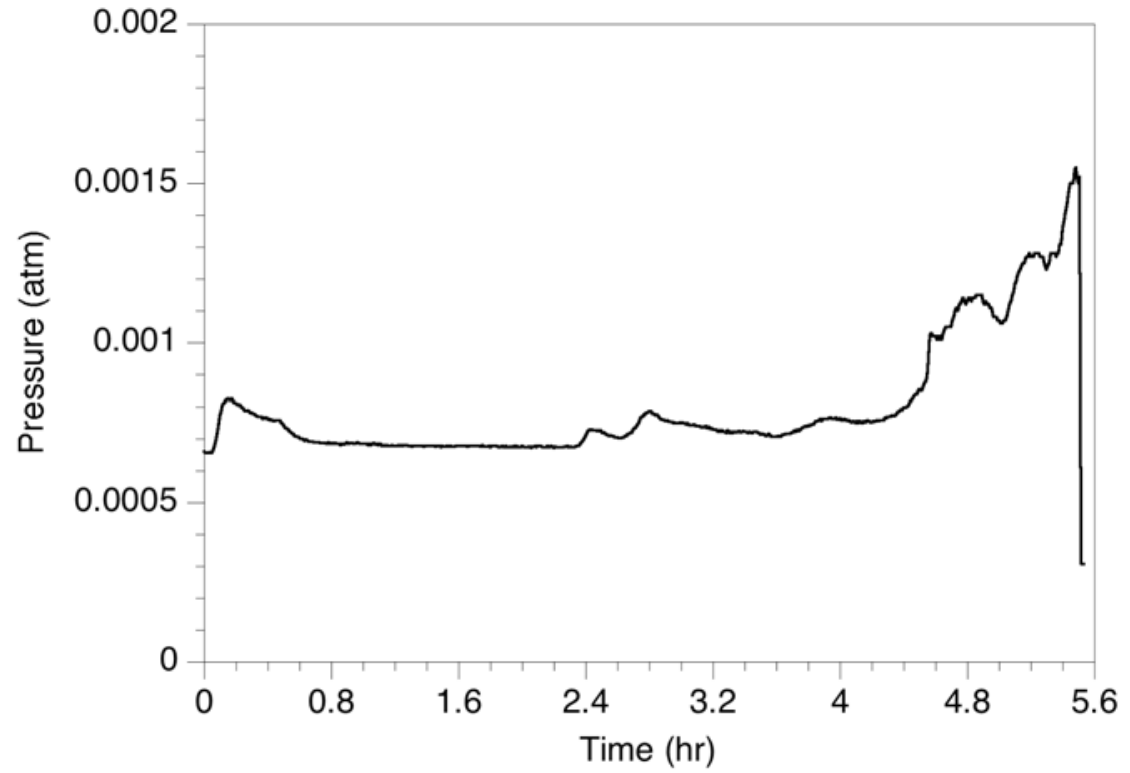

Fig. 2.9. Vacuum chamber pressure for Shot 6. (Data provided by $D X-2$.) 


\section{Analysis of the Large-Scale Annular Cookoff Experimental Results}

The results of the LSAC experiments allow us to draw several interesting conclusions about the behavior of PBX-9501 when it is subjected to slow-heating conditions. During the conceptual design of the LSAC assembly, it was originally thought that symmetric heating of a symmetric assembly would produce a symmetric initiation within the explosive. This would indeed be the case if the conductive heat flux throughout the assembly did not encounter spatial variations in the contact resistance between the HE, the metal liners, and the end caps. However, as was shown by the thermocouple records for all shots, perfectly symmetric heating was not attained, even before the internal heat generation within the HE was significant. We believe it was not realized because of azimuthal and axial variations in the contact resistance, which, in turn, depend on the contact pressure between the metal and the HE. In contrast to symmetric heating, asymmetric heating necessarily gives rise to an asymmetric temperature distribution, where the hottest region will become hotter as a result of the decomposition kinetics of PBX-9501.

This information was also used in the development of a post-ignition model, the results of which will be discussed in detail in Chapters 5 through 10.

\subsection{Ignition Time and Location in the Large-Scale Annular Cookoff Assembly Shots}

For each test, the time to ignition, or thermal runaway, was obtained from the thermocouple data and is shown in Fig. 3.1. Even though we have only a small sample with which to perform a statistical analysis, it is readily apparent that the casing material of the LSAC assembly plays an important role in determining the time to ignition when PBX-9501 is subjected to cookoff conditions.

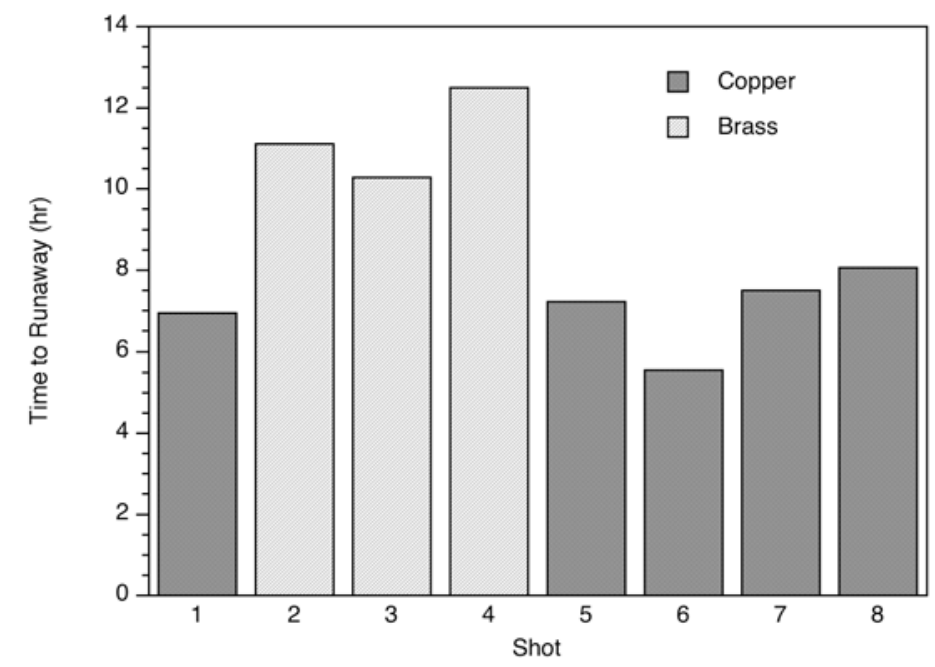

Fig. 3.1. Time to ignition for the LSAC shots. (Based on data provided by DX-2) 
Differences in the time to ignition among the shots cannot be explained by the difference in the thermal properties between copper and brass. Their corresponding thermal diffusivities can account only for discrepancies in thermal behavior on time scales of seconds but not of hours. The size of the assembly does not appear to significantly affect the time to ignition either. But according to the results shown in Fig. 3.1, the average thermal runaway time for the tests having a brass casing is 11.30 hours, whereas the corresponding time for tests with a copper casing is 7.06 hours. On average, it took $60 \%$ more time for thermal runaway to occur in assemblies with brass casings than in those with copper. Because it has been shown experimentally that confinement affects the time to ignition for PBX-9501 under slow heating conditions, (i.e., more confinement or sealing decreases the time to ignition [Kaneshige et al. 2003]), one can speculate that perhaps the assembly of the brass casing was less confined than the corresponding assembly of the copper casing. However, because of the lack of documentation (detailed design drawings, assembly tolerances, etc.) about the LSAC tests, the actual reason for the difference in the time to ignition between brass and copper casing materials may never be known. It would be desirable to conduct a series of smallscale experiments in the near future to address the potential effect of the casing material.

In general, the locations where the thermocouple data give the highest temperatures roughly coincide with the ignition regions. Figure 3.2 shows the angular location of the thermocouple that measured the highest temperature and then compares it with the apparent angular location of the thermal runaway, as inferred by high-speed photography, contact wires, optical fibers, or other available data. For example, when relying on highspeed photographs, the angular location of the ignition region was estimated by locating the largest wall displacement of the inner liner.

Shots 1, 5, and 7 were not included in Fig. 3.2 because no other data were available, except for the thermocouple data from which to infer the location of the ignition region. In Shot 4, there appear to be two initiation regions, as shown in Fig. 3.11, so there is a data point missing for this shot in Fig. 3.2. Also in Shot 4, two thermocouples at the same angular location failed, which coincidentally were located near the second initiation region. As indicated in Fig. 3.2, when it was possible to infer the location of the initiation region, this location was very close to where the thermocouple detected the highest temperature. However, the thermocouples were uniformly distributed every $60^{\circ}$, so the location at which the highest temperature was recorded may have been within $\pm 30^{\circ}$ of where the inferred ignition began. 


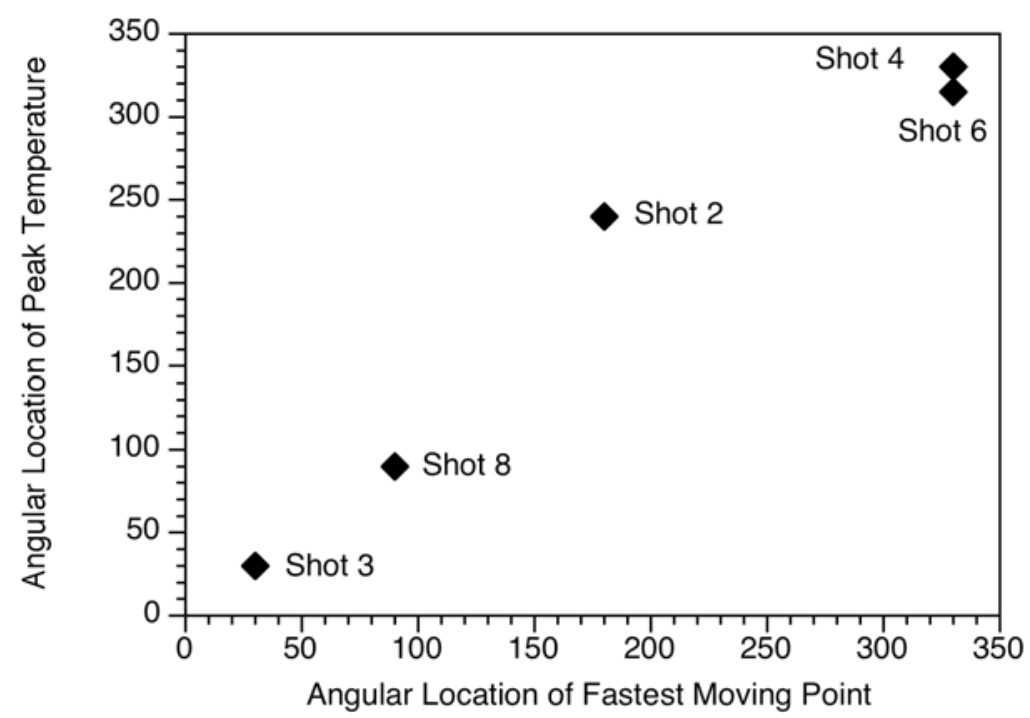

Fig. 3.2. Angular location (in degrees) of the peak temperature sensor vs. angular location (in degrees) of thermal runaway for five LSAC tests. (Based on data provided by $D X-2)$

\subsection{Inner-Liner Fastest Moving Point}

High-speed photographs in which the field of view was not obscured by what we believe were gaseous products were digitized and used to estimate the inner-liner velocity as a function of angular location. In Shot 6, the position of the fastest moving point (FMP) on the inner liner can be traced from $0 \mu$ s up to $70 \mu$ s. Figure 3.3 shows the first photograph of Shot 6 , which was taken $30 \mu$ s after one of the four diagnostic trigger wires was activated by the inner-liner motion. Since the motion of the wall is only observed between $260^{\circ}$ and $360^{\circ}$, this implies that the ignition began somewhere within this sector. Although the axial location of the ignition point or region cannot be determined from this image, it most likely originated at the angular location where the liner had the greatest radial displacement, that is, at the FMP where the velocity vector points radially inward at approximately $315^{\circ}$.

Figure 3.4 shows the normalized radial displacement of the FMP, taken from the photographs of Shot 6 , as a function of time. By taking the time derivative of the fitted curve (dotted line), with respect to time, the velocity of the FMP can be estimated. This velocity, shown in Fig. 3.5, has been extrapolated back in time by drawing a straight line backward from $30 \mu \mathrm{s}$, using the same slope as the velocity curve at that point (shown as a dotted line in the figure). From this extrapolation, we can estimate that burning started about $-40 \mu \mathrm{s}$ from the experimental zero time. We remind the reader that in Fig. 3.5, the time origin is taken to be the instant when the inner liner contacted the contact wire closest to the $315^{\circ}$ region and that this contact wire was initially at a radial distance of approximately $1 \mathrm{~mm}$ from the inner liner. 


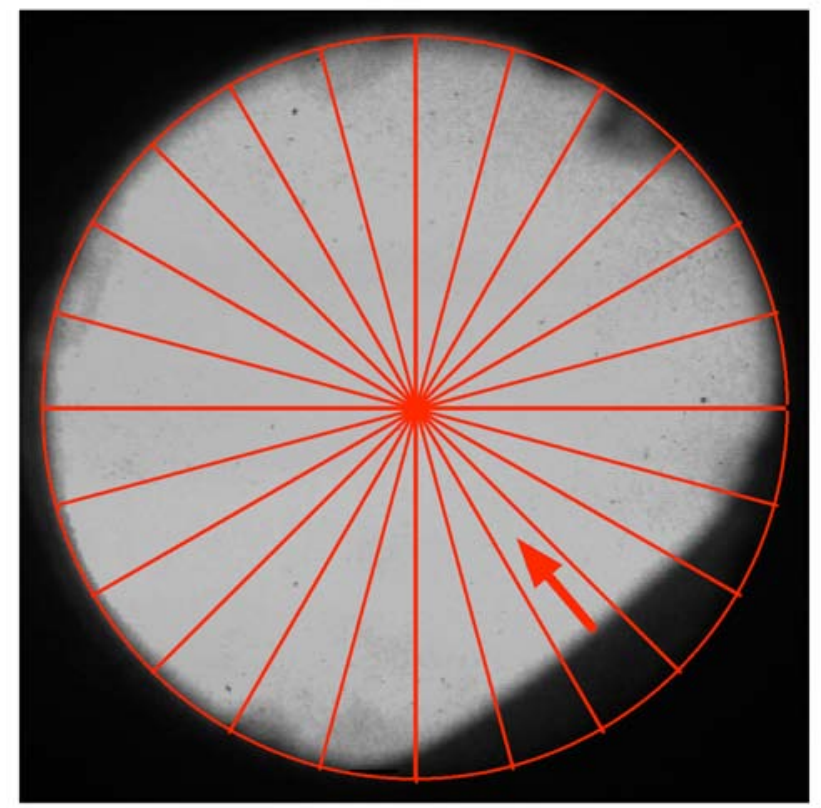

Fig. 3.3. Location of the FMP velocity vector at $30 \mu \mathrm{s}$ in Shot 6. (Based on photo provided by $D X-2)$

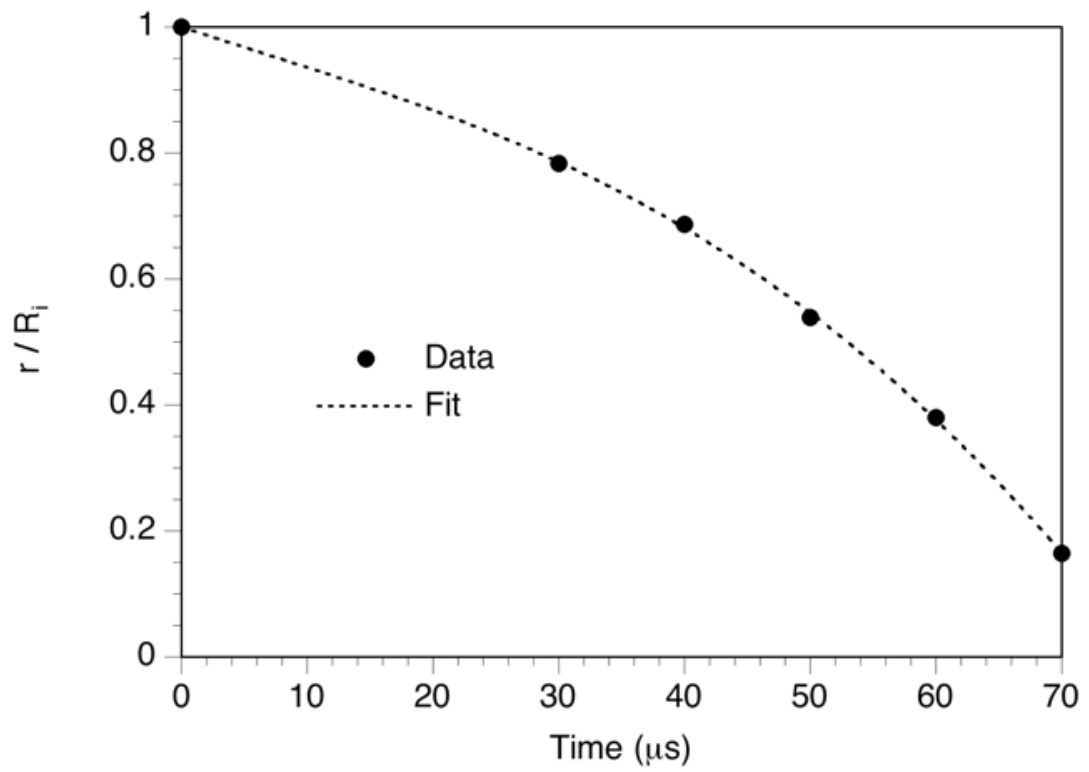

Fig. 3.4. Normalized radial displacement of the FMP, taken from the photographs of Shot 6. (Based on photos provided by DX-2) 


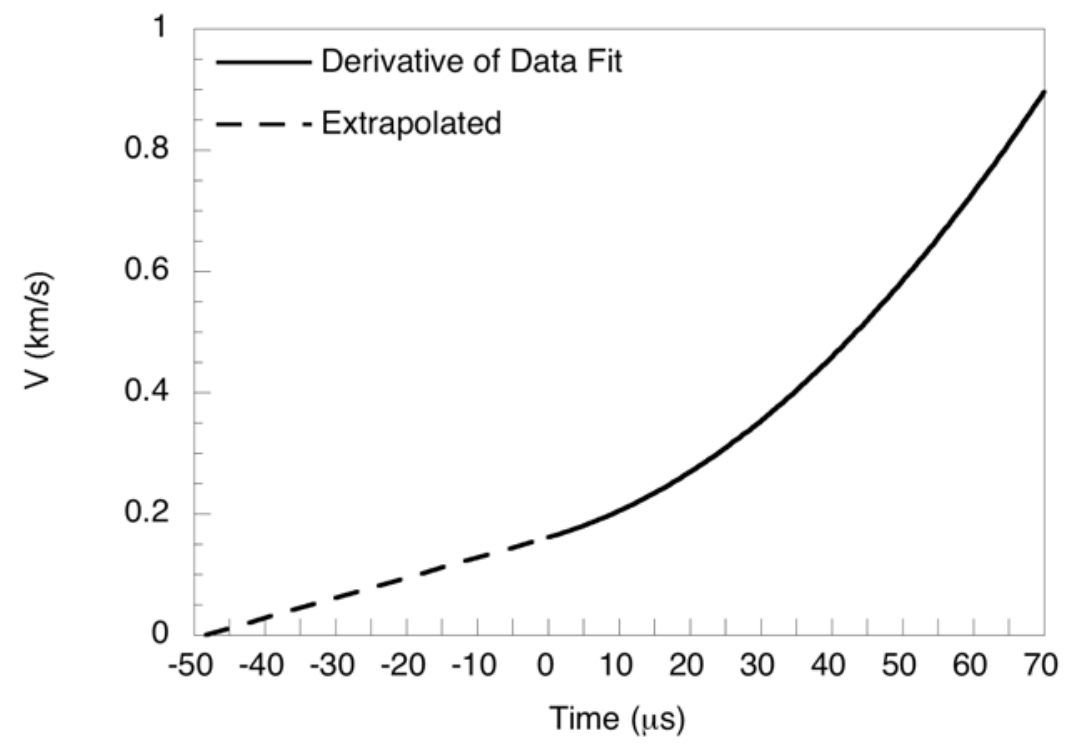

Fig. 3.5. Speed of the FMP of the liner in Shot 6.

The FMP is accelerated by gaseous products released by the chemical reaction after thermal runaway. Its speed is $0.36 \mathrm{~km} / \mathrm{s}$ at $30 \mu \mathrm{s}$ and $0.9 \mathrm{~km} / \mathrm{s}$ at $70 \mu \mathrm{s}$; at $30 \mu \mathrm{s}$, the FMP has traveled approximately $0.82 \mathrm{~cm}$.

In addition to the analyses described for Shot 6, we performed data analyses and simulations on Shots 3 and 4. Because less data were available for these shots, our analyses were not as extensive as those for Shot 6. Figure 3.6 shows the sequence of photographs that reveal the collapsing inner liner of Shot 3. At $10 \mu \mathrm{s}, 15 \mu \mathrm{s}, 20 \mu \mathrm{s}$, and $25 \mu \mathrm{s}$, it is easy to distinguish a clear-cut boundary that delineated the inner liner. At 30 $\mu s$, the metal liner can be equally well discerned except for a small sharp streak and a bulge. From these photographs, we estimated the velocity of the FMP (azimuthally located at about $173^{\circ}$ ) and in Fig. 3.7 compare it with that of Shot 6 (Fig. 3.5). At $30 \mu \mathrm{s}$, the continuity in the estimated velocities (following the FMP at a clear-cut boundary) between these two shots suggests that the metal liner was accelerated at similar levels in both cases. Our interpretation of what occurred beyond $30 \mu \mathrm{s}$ in Shot 3 is that the field of view was obscured by gaseous streaks from the deflagration products, which prevented us from tracking the inner liner unambiguously. These gases appeared after the end caps failed (i.e., failed to contain the gaseous products at high pressure). 


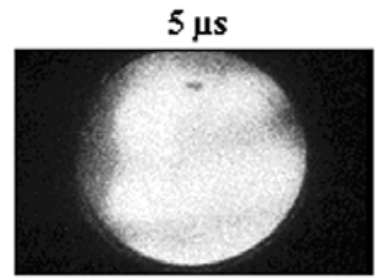

$20 \mu s$

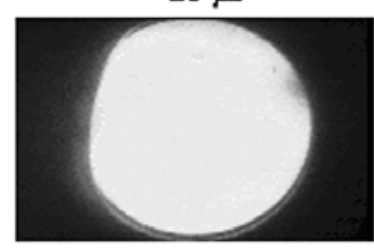

$35 \mu s$

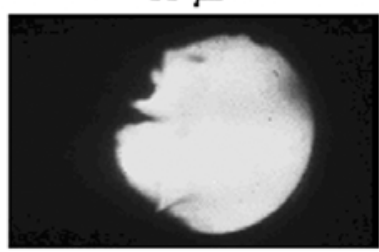

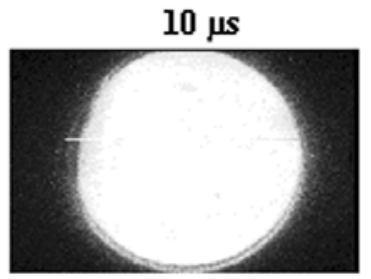

$25 \mu s$

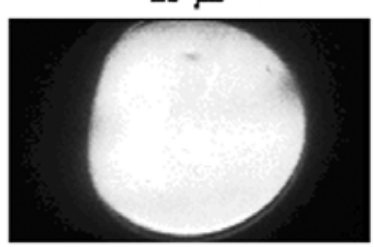

$40 \mu s$

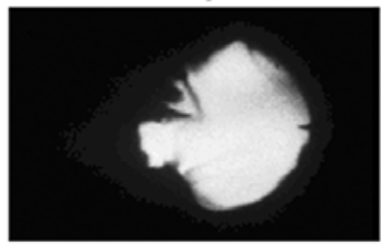

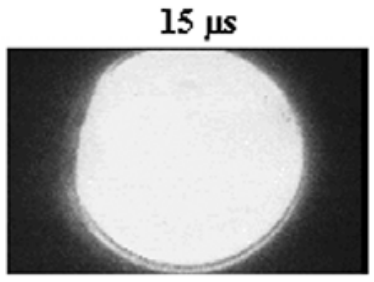

$30 \mu s$

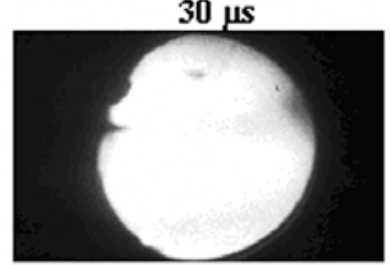

$50 \mu s$

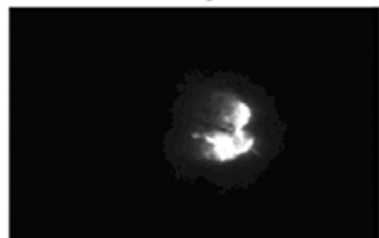

Fig.3.6. Sequence of photographs taken of the collapsing inner liner in Shot 3. (Data provided by $D X-2$.

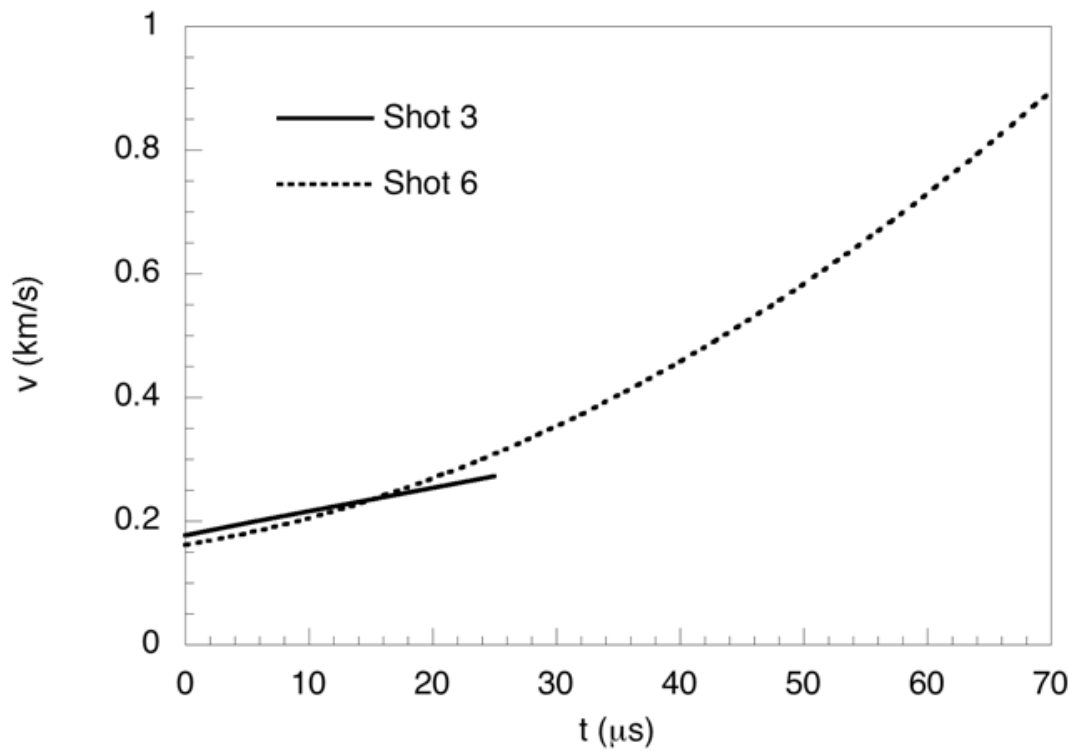

Fig. 3.7. Comparison between the estimated velocities of the FMPs in Shots 3 and 6. 
In Shot 3, the end caps probably failed at about $30 \mu$ s (Fig. 3.6), whereas in Shot 6, they probably failed after $70 \mu \mathrm{s}$ (Fig. 2.4). This interpretation differs from that offered by Asay et al. (1999), who argue that the streaks observed in the photos from $30 \mu$ s through $50 \mu$ s are metal and not gases. As demonstrated in Shot 8, the confinement provided by the end caps in the LSAC design was relatively weak. It took the reaction of only a small amount of explosive (believed to be about $15 \mathrm{~g}$ ) to remove both end caps, as can be seen in Fig. 3.8. Failure of the casing confinement is further suggested in Shot 6 by examining the RW data shown in Fig. 2.5. In this case, the strain increases from approximately $50 \mu \mathrm{s}$ to $80 \mu \mathrm{s}$, then decreases from $85 \mu \mathrm{s}$ to $100 \mu \mathrm{s}$, and then increases again up to 150 $\mu$ s. The sudden decrease in the strain at the outer liner, measured by the RWs between 80 $\mu \mathrm{s}$ and $100 \mu \mathrm{s}$, may be due to elastic unloading caused by the rapid release of gaseous products from the deflagration.

In Shot 3, if one assumed that what we believe is a gas streak (at $165^{\circ}$ in the $30 \mu \mathrm{s}$ photograph) was indeed metal, then the inferred velocity of the "streak tip" up to $50 \mu \mathrm{s}$ would have two velocity extrema, as shown in Fig. 3.9. Notice that these streaks, also shown in Fig. 3.6, are surrounded by shade gradients; this is particularly pronounced in the frame taken at $50 \mu \mathrm{s}$. However, it is possible to render an image with a different contour by adjusting the brightness and contrast in some of these images. Notice how the duck-shaped, bright region in the center of the image on the left (Fig. 3.10) can be increased (as shown on the right), resulting in a shade gradient that is different from that on the left.

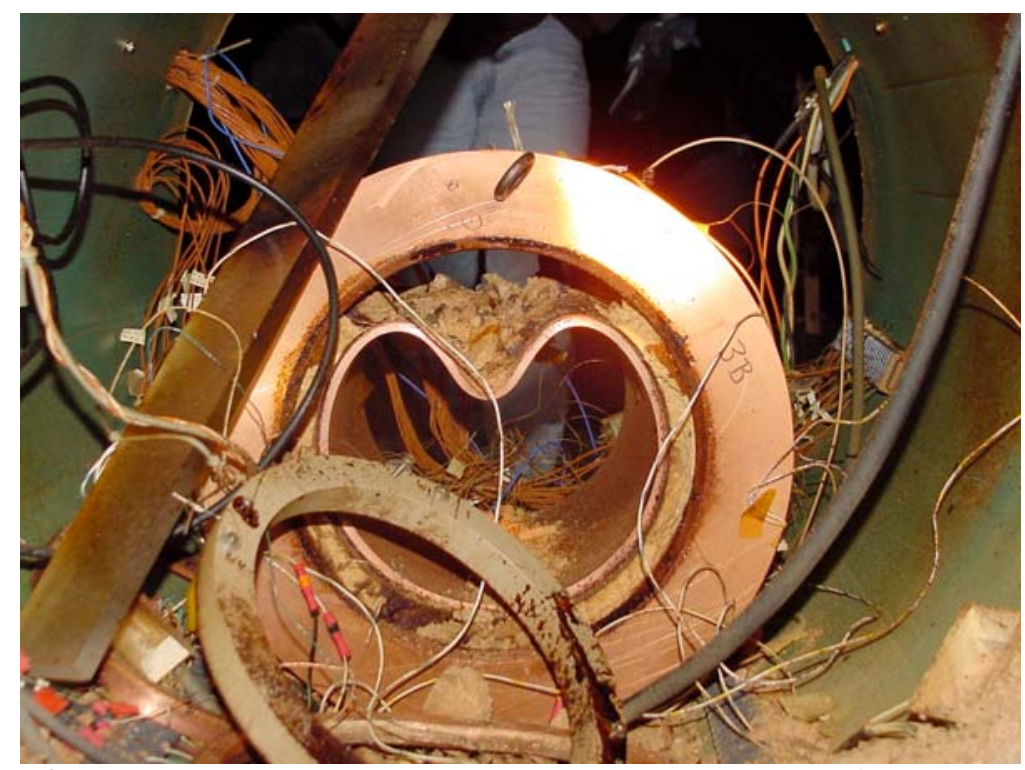

Fig. 3.8. Shot 8 after only a small portion of the HE ignited. (Photo provided by $D X-2$. 


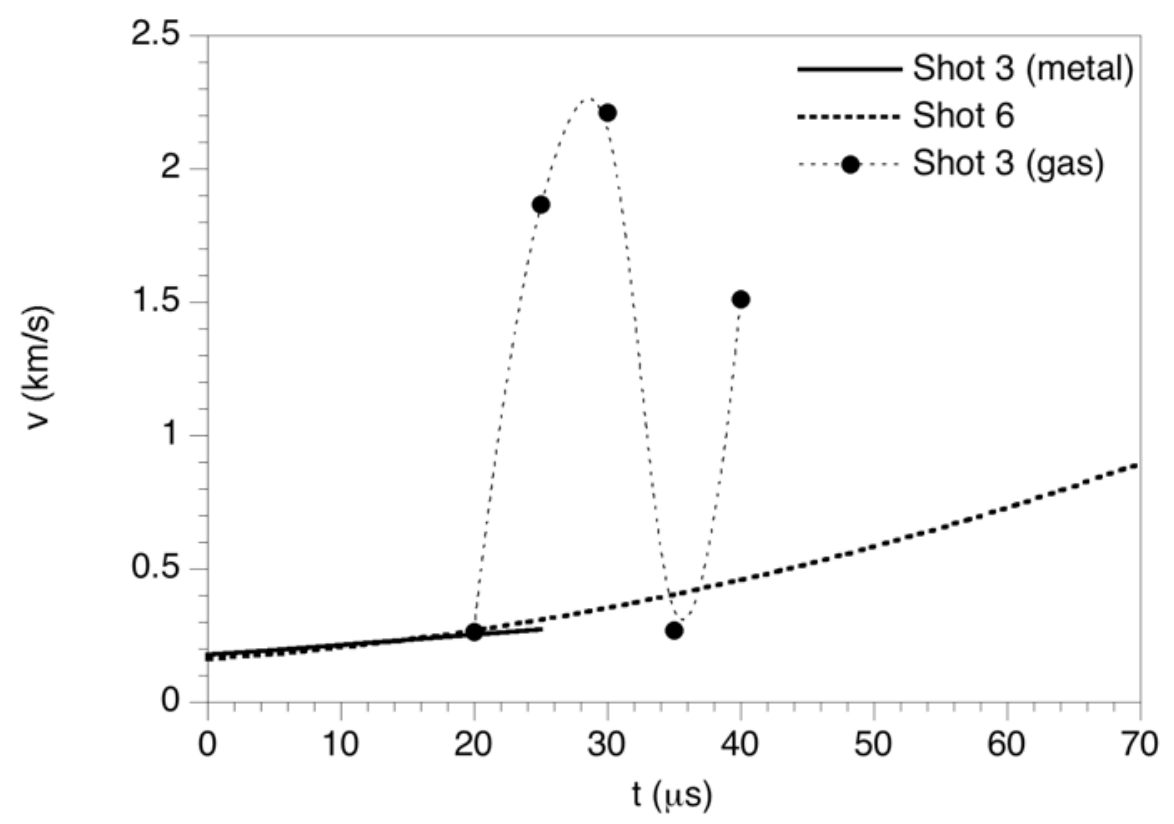

Fig. 3.9. Inferred velocity of the "streak tip" at $165^{\circ}$ in Shot 3 .
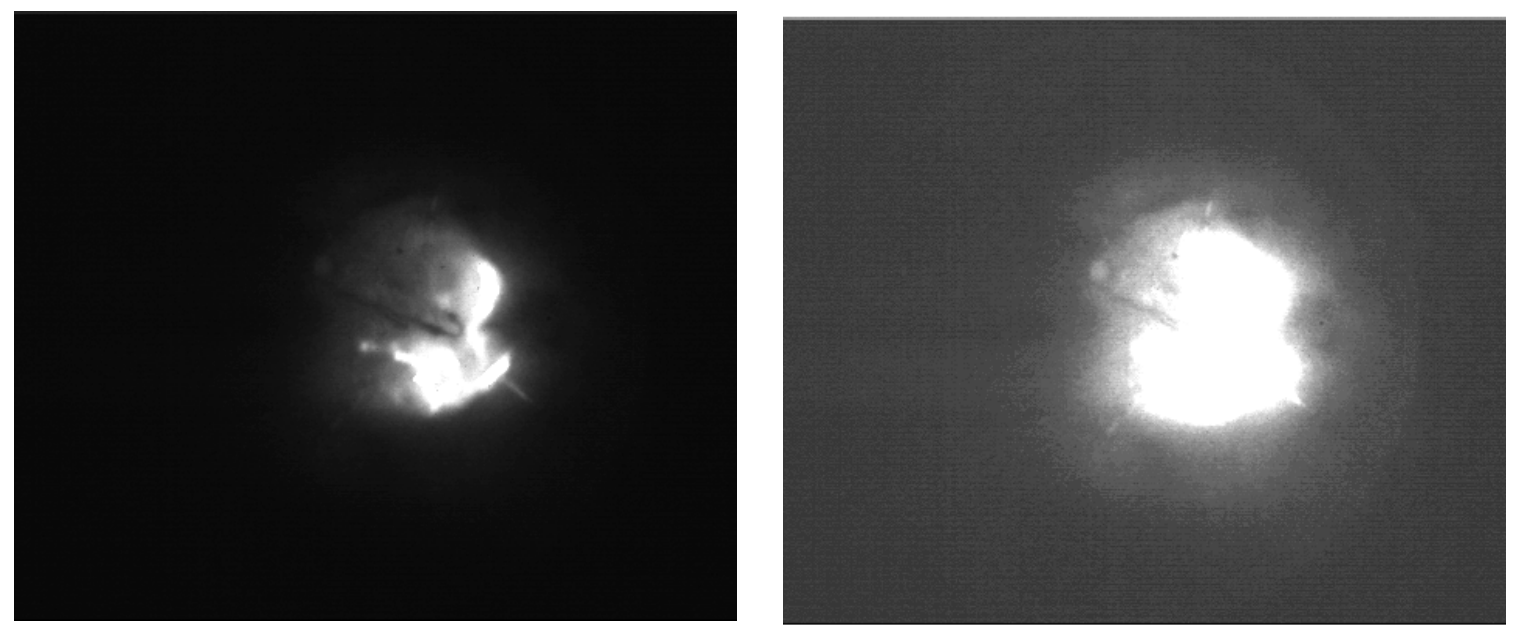

Fig. 3.10. Effect on the image after adjusting the brightness and the contrast in the frame at $50 \mu \mathrm{s}$ in Shot 3. (Based on data provided by $D X-2$ )

The shade gradient near the duck-shaped contour was effectively shifted outward, although no change was detected in the shade gradient farther from the center of the image. This kind of uncertainty would be removed if x-ray images were available (x-ray diagnostics were implemented in all of the shots but did not produce useful images for analysis). From the photographs in Fig. 3.6, it was concluded in Howe et al. (2000b) that "These frames show a remarkably high degree of symmetry in the collapse process." This interpretation stemmed from the assumption that everything appearing dark in the photograph was part of the metal liner. Based on a metric for symmetry, we show in Chapter 6 that the evidence collected from the first five photographs of Shot 6 in Fig. 2.4 
strongly indicates that the collapse of the inner liner was inherently asymmetric (similar results are obtained for the first $25 \mu$ s in Shot 3).

A striking feature unique to Shot 4 was the simultaneous ignition of two nearly diametrically opposed sites (one at $180^{\circ}$ and the other at $345^{\circ}$ ). This feature, although not apparent at a first glance, can be readily observed when a polar grid is overlaid onto the photographs. Figure 3.11 shows the last unambiguous frame available for Shot 4 at $70 \mu \mathrm{s}$. The inner liner on the right has advanced a distance slightly larger than that on the left. Based on the photographs at $50 \mu \mathrm{s}, 60 \mu \mathrm{s}$, and $70 \mu \mathrm{s}$, the estimated velocities of the FMPs at $180^{\circ}$ and $345^{\circ}$ during this short time interval $(20 \mu \mathrm{s})$ are shown in Fig. 3.12. Notice that while the FMPs at $345^{\circ}$ are moving slightly faster than at $180^{\circ}$, their traversed distances are roughly the same. The traversed distance of the FMP in Shot 6 at $30 \mu \mathrm{s}$ (Fig. 3.3) was $0.84 \mathrm{~cm}$ (22\% of the original radius of the inner liner $\left.R_{i}=3.81 \mathrm{~cm}\right)$; in Shot 4 at $70 \mu \mathrm{s}$, it was $0.6 \mathrm{~cm}\left(16 \%\right.$ of $\left.R_{i}\right)$. In Shot 6, the FMP was moving at about $0.4 \mathrm{~km} / \mathrm{s}$, and in Shot 4, the average velocity of the FMPs was about $0.6 \mathrm{~km} / \mathrm{s}$. Therefore, during the early stages of collapse, more work per unit time per unit volume was released by the gaseous products of deflagration onto the inner liner in Shot 4 than in Shot 6. This might indicate a higher degree of reaction violence in Shot 4 when compared with Shots 3 and 6. Table 3.1 summarizes the estimated FMP velocities in Shots 3, 4, and 6 for a liner displacement in the neighborhood of $0.6 \mathrm{~cm}$.

Table 3.1. FMP Velocities in Shots 3, 4, and 6

\begin{tabular}{|c|c|c|}
\hline Shot & Liner Displacement $(\mathrm{cm})$ & Velocity $(\mathrm{km} / \mathrm{s})$ \\
\hline 3 & 0.6 & 0.3 \\
\hline 4 & 0.61 [left] 0.58 [right] & 0.8 [left] $\quad 0.5$ [right] \\
\hline 6 & 0.6 & 0.3 \\
\hline
\end{tabular}




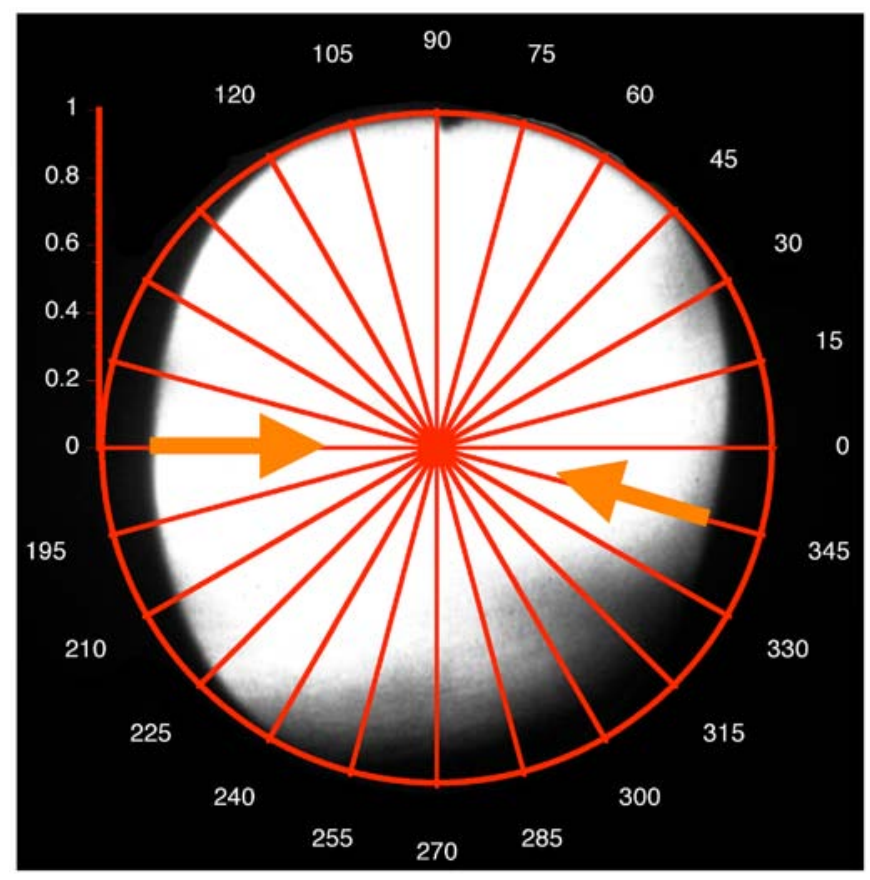

Fig. 3.11. Location of the FMP velocity vectors at $70 \mu \mathrm{s}$ in Shot 4 (grid thickness has been increased for clarity). (Based on data provided by $D X-2$ )

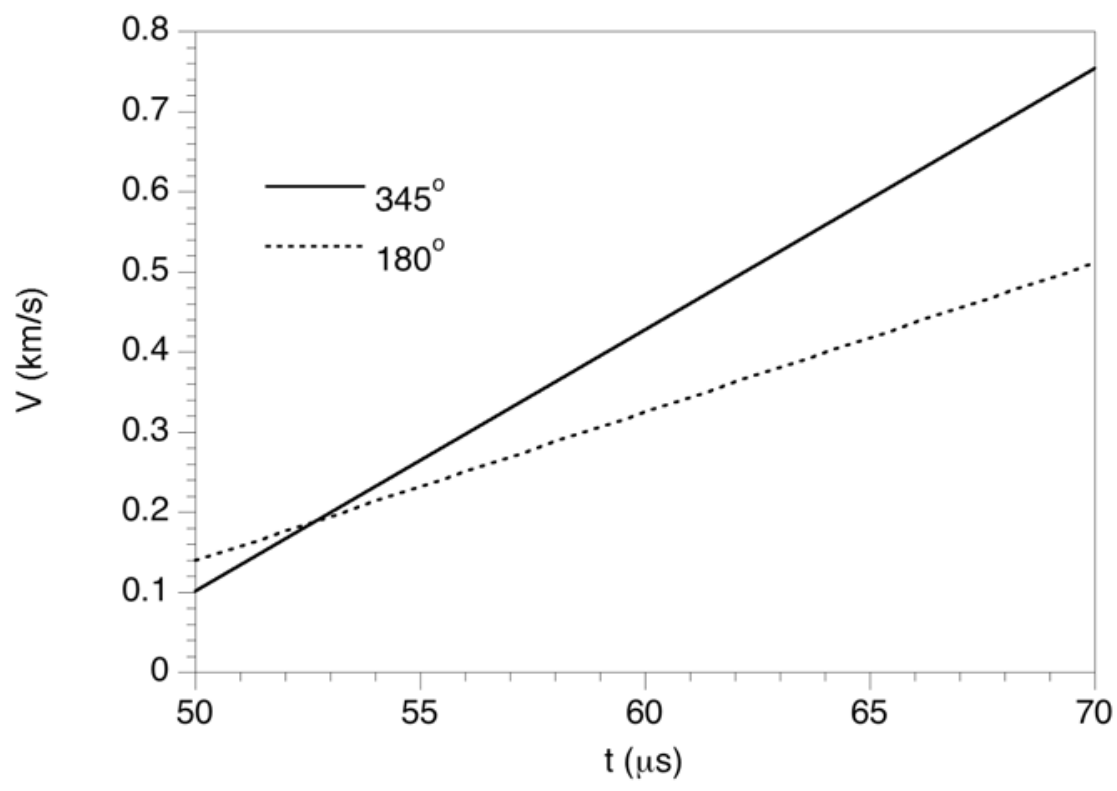

Fig. 3.12. Estimated velocities of the FMPs at $180^{\circ}$ and $345^{\circ}$ for Shot 4 . 


\subsection{Burn-Rate Estimate from Optical Fiber Data in Shot 4}

For Shot 4, a characteristic velocity of the reaction of cooked PBX-9501 was estimated from the optical fiber data in Fig. 2.8 and Table 2.4. These data were sorted according to the relative time (the time of arrival to a sensor with respect to the arrival time to the sensor at $0^{\circ}$ ) and the arc length (the outer radius, $7.94 \mathrm{~cm}$, of the explosive times the angular location of the sensor), as shown in Table 3.2 and Fig. 3.13.

Table 3.2. Time of Arrival at Subsequent Sensors with Respect to the $0^{\circ}$ Sensor

\begin{tabular}{|c|c|c|c|}
\hline $\begin{array}{c}\text { Relative } \\
\text { Time }(\boldsymbol{\mu s})\end{array}$ & \multicolumn{2}{|c|}{ Angular Location (radians) } & $\begin{array}{c}\text { Arc Length } \\
\text { (cm) }\end{array}$ \\
\hline 0 & $0^{\circ}$ & 0.0 & 0 \\
\hline 22 & $30^{\circ}$ & 0.52 & 4.16 \\
\hline 34 & $60^{\circ}$ & 1.05 & 8.31 \\
\hline 47 & $90^{\circ}$ & 1.57 & 12.47 \\
\hline 53 & $120^{\circ}$ & 2.09 & 16.63 \\
\hline
\end{tabular}

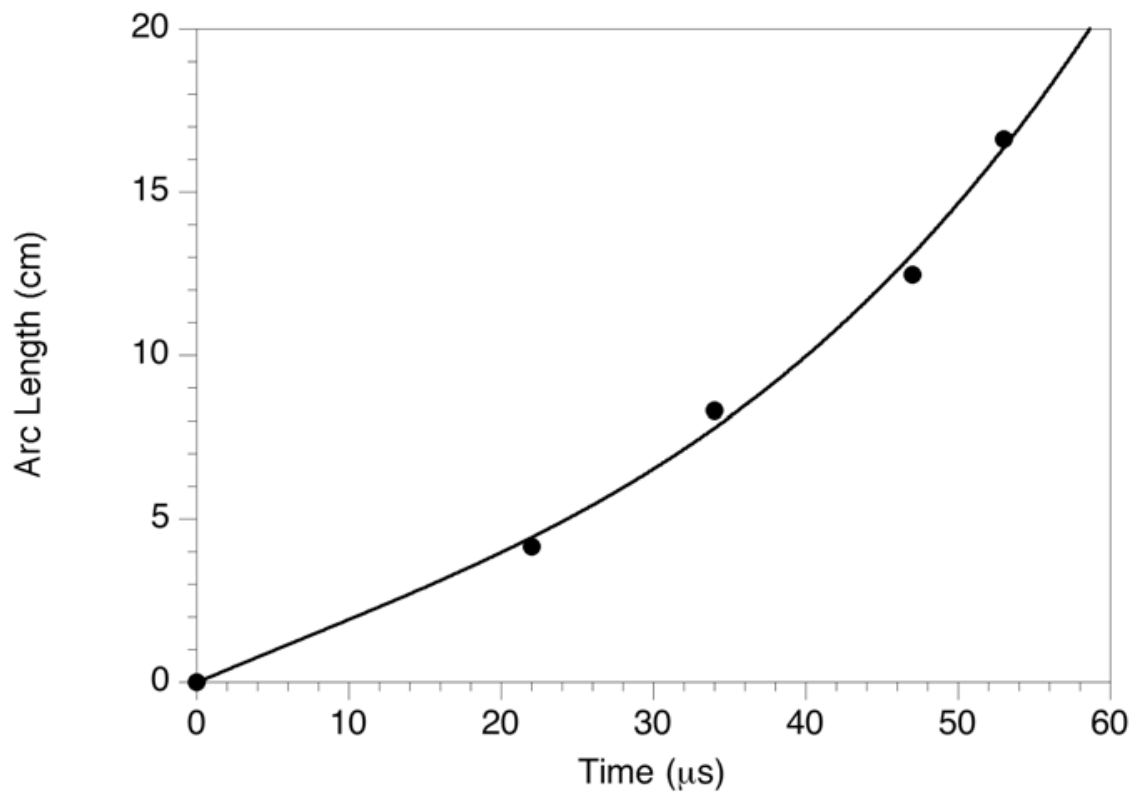

Fig. 3.13. Arc length as a function of time from the optical fiber data in Shot 4 .

Figure 3.14 shows the estimated burn rate inferred from the optical fiber data in Shot 4 , assuming that the wave front propagates circumferentially through the explosive. Note that there may be other possibilities for the propagation of the wave front, which may result in lower burn rates, but there is not enough information in the available experimental data to confirm or reject the assumption of circumferential wave-front propagation. In Figure 3.14, the burn rate at $50 \mu \mathrm{s}$ is approximately $5.4 \mathrm{~km} / \mathrm{s}$. This is an 
overly high value for a deflagrating front speed and a low value for a detonation, which is $8.8 \mathrm{~km} / \mathrm{s}$ for pristine PBX-9501. At $70 \mu \mathrm{s}$, when the liner wall on the left of Shot 4 (see Fig 3.11) advanced a distance of $0.61 \mathrm{~cm}$, it had a velocity of $0.76 \mathrm{~km} / \mathrm{s}$. This velocity is more than twice as fast as that for the FMPs on the liners in Shots 3 and 6 (see Table 3.1).

Figure 3.15 shows the contours of the normalized data from the optical fibers where the brightest regions correspond to peak values. Notice that at about $130 \mu$ s there are two distinct regions of high luminosity near $0^{\circ}\left(360^{\circ}\right)$ and around $180^{\circ}$, which shows that there were two propagating fronts.

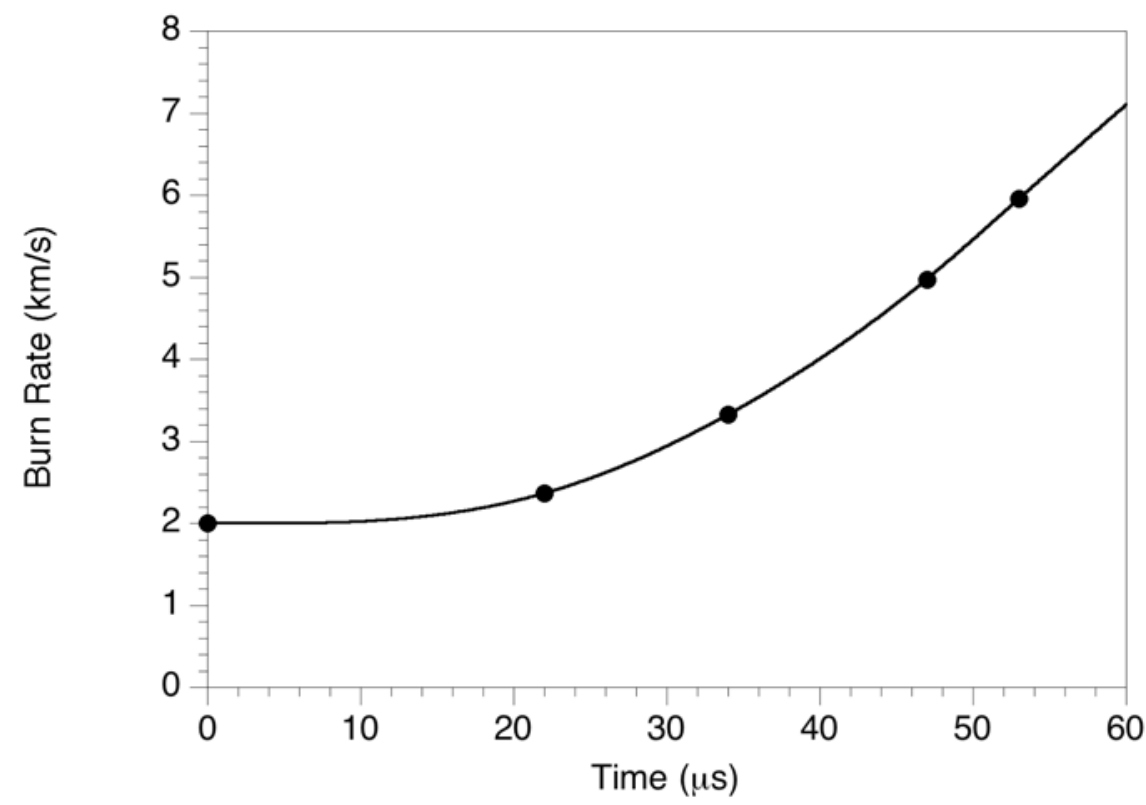

Fig. 3.14. Burn rate estimated from the optical fiber data in Shot 4. 


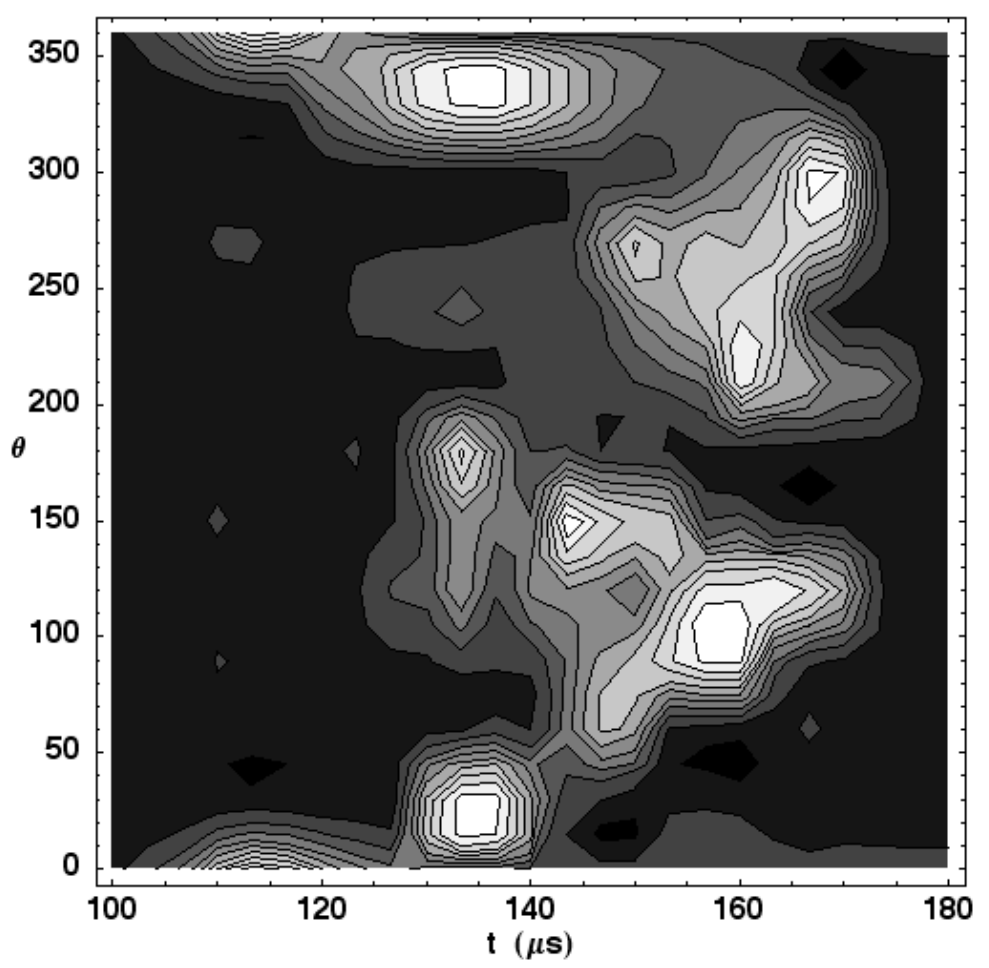

Fig. 3.15. Contours of normalized output from the optical fiber data in Shot 4 . 


\section{Pre-Ignition Hypotheses and Modeling of the Large-Scale Annular Cookoff Assembly}

Investigators at LLNL, SNL, and LANL have been working to develop models to simulate the thermal, chemical, and mechanical states that occur within PBX-9501 when it is subjected to cookoff conditions while it is confined by a metallic assembly. However, our present knowledge about the chemical kinetics of thermal decomposition in PBX-9501 requires that we make several simplifying assumptions about the thermophysical and thermochemical properties of the intermediate constituents and reduce the number of kinetic steps. Despite the complexity of thermal decomposition of energetic materials, firm steps have in fact been taken toward understanding the kinetics, and several simplified schemes have been proposed and applied to specific problems with some success (McGuire and Tarver 1981; Brill and Karpowicz 1982; Thynell et al. 1996; Henson et al. 1999; Dickson et al. 2000; Henson et al. 2002; Smilowitz et al. 2002 a-b, Behrens 2002; Tarver and Tran 2004). Recently, there has also been considerable effort to experimentally quantify the behavior of PBX-9501 when subjected to a wide range of thermal and shear-rate conditions (Tappan et al. 2002; Thompson et al. 2002; Kaneshige et al. 2002; Asay et al. 2003; Kaneshige et al. 2003; Smilowitz et al. 2003; Kaneshige et al. 2004; Maienschein et al. 2000; Maienschein et al. 2004; Explosives Reference Guide 2005 and references therein).

A practical cookoff post-ignition modeling capability that can be implemented in hydrodynamics codes requires an empirical equation of state for the gaseous products of deflagration. Detailed knowledge of the thermodynamic state at thermal runaway provides information about when and where ignition will occur. In establishing the thermodynamic state of the metal that is being propelled by the deflagration products, pre-ignition is not as important as the peak pressure reached after thermal runaway and the speed of the deflagration front. Even though a pre-ignition modeling capability is not a prerequisite for post-ignition modeling, in this chapter we examine recent data regarding hypotheses about pre-ignition that are claimed by some investigators to be relevant to post-ignition behavior.

The way in which heat is transferred throughout the $\mathrm{HE}$ will determine the time and location of ignition. Two approaches have been followed to model the transport of heat in pre-ignition simulations, namely, conductive heat transfer and conductive/convective heat transfer based on the hypothesis that the HE behaves as a homogenous porous medium obeying Darcy's law for a single gaseous phase. During the last four decades, the former approach, and its variants, have been used to model thermal runaway problems in general, which includes energetic materials. It is only very recently that the latter approach has been applied to model the pre-ignition phase of cookoff for PBX-9501.

A benchmark often used to validate pre-ignition models is the prediction of the timeto-ignition. At this time, it is not possible to have a truly predictive capability because there are phenomena that are not well understood and parameters that have not been measured. Therefore, simulations with existing models are used to "tune" or calibrate model parameters to match experimental results in the hopes that they might be applicable to real situations. This leads to a posteriori "predictions" where the experiment is performed first and the simulations follow. However, when the models are not robust 
enough, simulations with a set of parameters that can predict one experiment are unable to predict another with the same degree of accuracy. Therefore, a successful "prediction," by means of numerical simulations based on a given set of mathematical models, of a certain class of experiments does not imply that a predictive capability has been developed. Modelers who have followed either of the approaches mentioned above have been successful at "predicting" time-to-ignition. Parameter tuning can lead to agreement between numerical simulations and experiments that is within less than a fraction of $1 \%$, which is certainly less than the experimental variability. That the agreement with experiments can be astonishing in some simulations is not surprising, because there is a multitude of adjustable parameters, some of which are not known and thus can be varied arbitrarily.

If two different approaches can lead to the same answer, which one best describes reality? We cannot conclusively answer this question at the present time. However, existing data point out that there are difficulties with (1) the assertion that Darcy flow (flow through porous media) is a key heat transfer mechanism in the LSAC configuration during pre-ignition, (2) interpretation of the permeability measurement experiments, and (3) pre-ignition modeling approach in general. In current model development, the rheological and chemical behaviors of the binder and their effects on cookoff have been ignored or neglected. Within the range of temperatures applicable to cookoff conditions, Kaneshige et al. (2003) found evidence that the binder in PBX-9501 affects its kinetics. In spite of this, the arguments, hypotheses, and models based on gas convection within a porous matrix of HMX (as in PBX-9501) have overlooked the presence of the binder during pre-ignition. In the Darcy flow model developed by the Nuclear Design and Risk Analysis (D-5) group (Zerkle and Luck 2003), it is assumed that through the interstitial space within PBX-9501 gases can permeate unimpeded as if they were in a "dry" granular medium. However, at room temperature, PBX-9501 is an impermeable composite where the binder occupies about $8 \%$ of the total volume; during cookoff, the binder begins to melt at $80^{\circ} \mathrm{C}$. Therefore, at cookoff temperatures above $180^{\circ} \mathrm{C}$, one has a liquid within a densely packed array of HMX crystals and not a free permeating gas. Furthermore, in recent permeability experiments, we believe convective transport of liquid binder at $185^{\circ} \mathrm{C}$ has been recorded but not recognized as such.

In the following sections, we present arguments showing that models based on treating PBX-9501 as a gas-saturated porous medium during the pre-ignition stage, when temperatures are around $180^{\circ} \mathrm{C}$, are not supported by available experimental evidence.

\subsection{Gas Generation during Pre-Ignition}

Smilowitz et al. (2003) measured the mass-fraction decomposition rate of unconfined PBX-9501 at several temperatures; Table 4.1 summarizes these results. At $209^{\circ} \mathrm{C}$, their data indicate that less than $7 \%$ of the explosive decomposed in approximately 2.3 hours. The SNL confined cookoff hot-cell experiments (Renlund et al. 1998) and the instrumented thermal ignition (SITI) experiments at lower temperatures (Kaneshige et al. 2003) show lower levels of decomposition. SNL's hot-cell experiments indicate that when a confined PBX-9501 sample is heated to $190^{\circ} \mathrm{C}$ for 1 hour, there is no mass loss, and when the sample is heated to $200^{\circ} \mathrm{C}$, the mass loss is $1 \%$ (Renlund et al. 1998). The

pressures recorded within the SITI confinement are consistent with a small mass fraction 
of explosive being decomposed. These pressure measurements were made at a small expansion gap located between the HE and the aluminum casing. It appears that in the SITI experiments, gas evolves mostly from the HE surface because the time scales associated with other transport mechanisms of gas migrating from deep within the sample (Darcy bulk permeation flow or diffusion) are larger. Direct measurements of the amount of gas generated within the HE in a confined sample of PBX-9501 under cookoff conditions are not available. Experiments performed on small samples (single crystals) of pure HMX crystals have shown the formation of microbubbles at nucleation sites (Behrens 2003). The formation of gases within PBX-9501, where HMX particles are coated with the binder, will be strongly influenced by the physical and chemical interactions between the binder and the HMX. However, details of these interactions remain largely unknown.

Table 4.1. Mass Loss of Unconfined PBX-9501 (Adapted from Smilowitz et al. 2003)

\begin{tabular}{|c|c|c|}
\hline Temperature $\left({ }^{\circ} \mathbf{C}\right)$ & Mass Loss (\%) & Time (hours) \\
\hline 180 & 5 & 24.23 \\
\hline 209 & 7 & 2.30 \\
\hline 231 & 54 & 3.85 \\
\hline 246 & 98 & 2.03 \\
\hline
\end{tabular}

In summary, there has been no indication or experimental proof of complete binder "gasification" that can lead to free gas permeation through the HE matrix at temperatures below $200^{\circ} \mathrm{C}$. As evidenced in micrographs, the molten binder resolidifies when a sample of PBX-9501 that has been heated to $180^{\circ} \mathrm{C}$ is cooled back down to room temperature (Berghout et al. 2002); thus, the interstitial space remains mostly filled with liquefied binder rather than gas.

\subsection{Binder Behavior during Pre-Ignition}

PBX-9501 contains 5\% wt. of binder made up of 2.5\%wt. Estane and 2.5\%wt. BDNPA/F (a plasticizer made up of equal parts of bis(2,2-dinitropropyl)acetal (BDNPA) and bis(2,2-dinitropropyl)formal (BDNPF)). There are many different kinds of Estane (a polyurethane thermoplastic) produced by B. F. Goodrich, and different authors report two kinds that have been used in the production of PBX-9501 (Palmer et al. 1993; Cady et al. 2000; Cooper 1996). For example, in Los Alamos Explosive Property Data (1980), it is reported that Estane 5703 is used in $\mathrm{PBX}-9501$ and that "Above $50^{\circ} \mathrm{C}$, the polyester polyurethane Estane depolymerizes, so its strength decreases with time." In the LLNL Explosives Reference Guide (2004), it is reported that Estane 5702 is used in PBX-9501. The melting temperatures of Estane 5702 and 5703 are $79^{\circ} \mathrm{C}$ and $77^{\circ} \mathrm{C}$, respectively, according to the "Estane Technical Data Sheet" (2001). In general, all thermoplastic polyurethanes are pumped and transported, in processes such as injection molding, over a wide range of temperatures between $177^{\circ} \mathrm{C}$ and $232^{\circ} \mathrm{C}$ (the self-ignition temperature of most Estane systems is about $393^{\circ} \mathrm{C}$; see "Estane Material Safety Data Sheet" 2001). 
BDNPA/F forms a eutectic that facilitates its use at low temperatures (Provatas 2000). The melting point of BDNPA/F is about $15^{\circ} \mathrm{C}$ (Rivera 1973). Therefore, even though the rheological behavior for the combination of Estane and BDNPA/F as a function of temperature (between $100^{\circ} \mathrm{C}$ and $200^{\circ} \mathrm{C}$ ) is not yet known, the binder will be in liquid form during cookoff conditions. At $204^{\circ} \mathrm{C}$, the apparent viscosity of pure Estane is reported to be 92 Pa-s ("Estane Technical Data Sheet" 2001). However, in the binder, Estane is diluted with $50 \%$ of BDNPA/F, which has a much lower viscosity than Estane and thus the viscosity of the polymer blend is expected to decrease. We are not aware of a rheological characterization of the binder as a function of temperature. The binder occupies $7.2 \%$ of the volume of PBX-9501 at room temperature. This volume will change during cookoff because of the HMX phase transition and the thermal expansion of the binder itself. Nonetheless, there will be liquid binder occupying the interstitial space within the HE. Therefore, at some point during cookoff, there will be a liquid phase in the HE, and the assumption of gas flowing through and around a bed of "dry" HMX crystals does not seem realistic.

Furthermore, according to a thermogravimetric analysis (TGA) of heated PBX-9501 binder (Fig. 4.1), the BDNPA/F is the first component to decompose. At about $200^{\circ} \mathrm{C}$, $48 \%$ of BDNPA/F has decomposed, corresponding to a $1.2 \%$ decomposition of the total mass in PBX-9501 (neglecting chemical interactions between the binder and the HMX crystals). However, the Estane remains in liquid form blended with 52\% of BDNPA/F, the former without noticeably decomposing until temperatures exceed $300^{\circ} \mathrm{C}$. Although the TGA conditions are not the same as those in a cookoff environment (where temperatures were maintained around $190^{\circ} \mathrm{C}$ ), the trends of decomposition are expected to be qualitatively similar. While the exothermic reaction proceeds toward runaway, the small amount of hot gaseous products generated by thermal decomposition (possibly distributed within the $\mathrm{HE}$ as bubbles localized at nucleation sites) interact with HMX crystals and a molten binder.

In order for the gases to flow through a porous bed of HMX crystals (as implied in the Darcy-flow hypothesis), the binder (mostly Estane with some fraction of BDNPA/F) would have to be extruded quickly. However, there is no experimental evidence of rapid and complete extrusion of all the binder in samples heated up to $186^{\circ} \mathrm{C}$ (Asay et al. 2004). It would also be unlikely that a pore network could develop between the HMX crystals and provide a pathway through which only decomposition gases would migrate while the molten binder remained motionless. Finally, it is unlikely that the decomposition product gases adsorbed by the binder would be transported efficiently. 


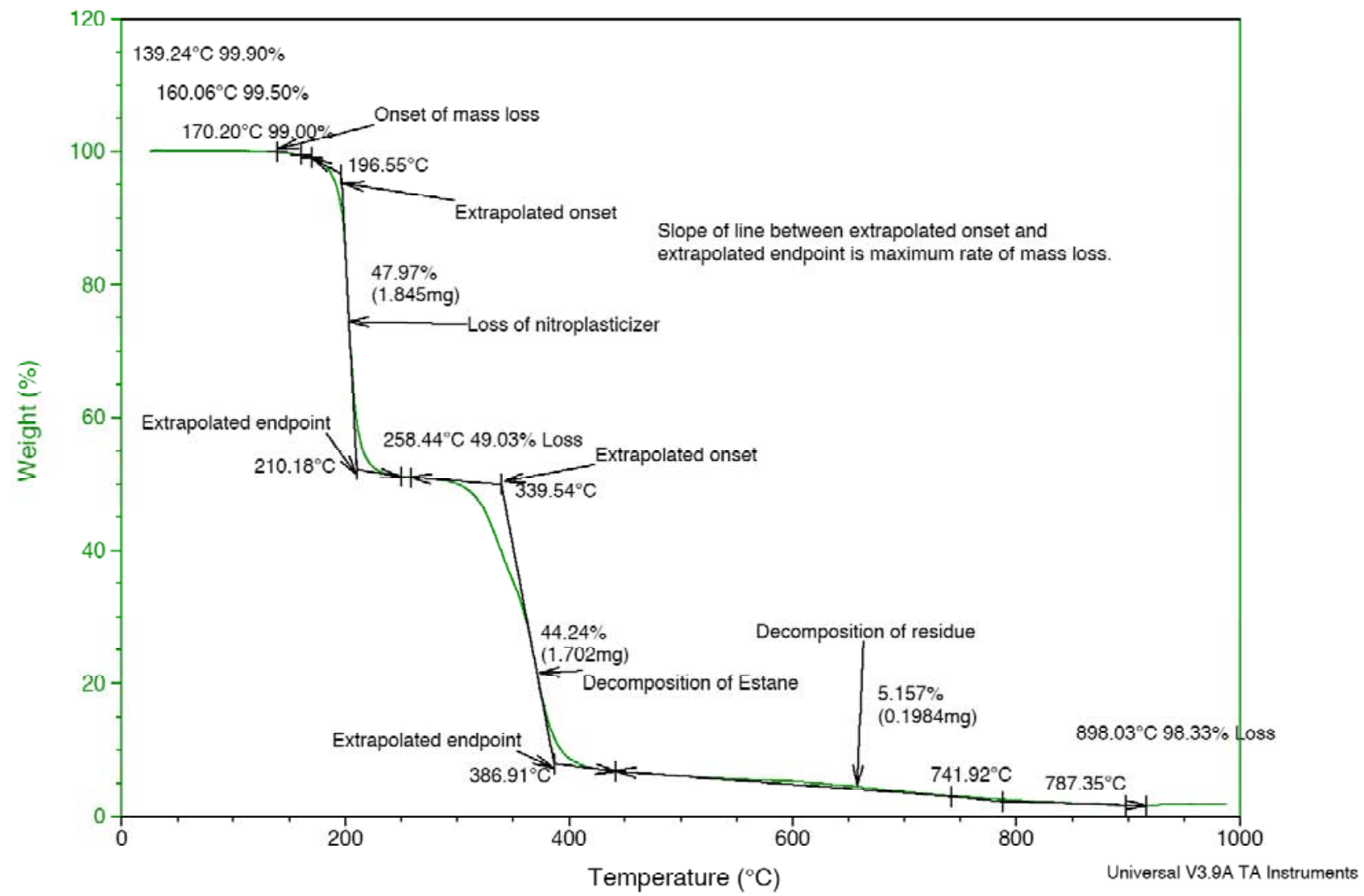

Fig. 4.1. TGA for the binder of PBX-9501. (Data provided by DX-2.)

\subsection{Permeability Measurements}

Experimental measurements of permeability in a porous material are performed with an apparatus called a permeameter. Based on the Darcy-flow model, the permeability of the medium (measured in units of $\mathrm{m}^{2}$ ) is independent of the fluid used in the measurement. There are several designs of this device, their use depending on the nature of the sample and whether the working fluid used in the measurement is a liquid or a gas. A permeameter design for measuring the permeability of a gas through a porous medium is depicted in Fig. 4.2. Gas under high pressure is forced through an open-ended cylinder (such as a pipe) filled with a porous material. The permeability of the sample can be inferred from the decrease of pressure within the original gas container. Typical values for common materials are $10^{-14} \mathrm{~m}^{2}$ for brick, $10^{-10} \mathrm{~m}^{2}$ for sand, and $10^{-9} \mathrm{~m}^{2}$ for a cigarette (Nakayama 1995). 


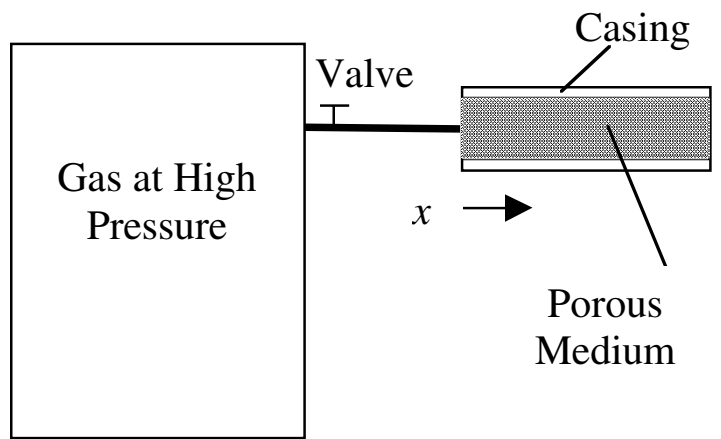

Fig. 4.2. Schematic depiction of a simple gas permeameter apparatus.

Consider a single-phase fluid flowing through an isothermal and chemically inert porous sample (e.g., air flowing through a brick). We refer to these conditions as the "normal Darcy conditions." If these conditions are applied to the permeameter in Fig. 4.2, we can use the ideal gas EOS and a mass balance to show that the rate of change of pressure in the tank is

$$
\frac{d p_{T}(t)}{d t}=-\frac{A_{s}}{V_{T}} u(x, t) p(x, t),
$$

where $p_{T}$ is the pressure within the tank, $V_{T}$ is the tank volume, $A_{s}$ is the cross-sectional area of the porous sample, $u(x, t)$ is the Darcian velocity of the gas flowing through the sample, $p(x, t)$ is the pressure within the sample, $x$ is the spatial coordinate within the sample, and $t$ is time.

For a one-dimensional, quasi-static plug flow in which inertial forces can be neglected, the momentum equation for Darcy flow is

$$
\frac{\partial p(x, t)}{\partial x}=-\frac{\mu}{k} u(x, t),
$$

where $\mu$ is the gas viscosity and $k$ is the permeability of the sample. At the sample ends, the boundary conditions are

$$
\begin{gathered}
p(0, t)=p_{T}(t) \\
p(L, t)=p_{0},
\end{gathered}
$$

where $L$ is the length of the sample and $p_{0}$ is the atmospheric pressure.

An expression for permeability is obtained by combining Eqs. (4.1) and (4.2), integrating with respect to $x$, applying the boundary conditions of Eqs. (4.3) and (4.4), evaluating at $x=0$, and solving for $k$ (see Appendix B for details) we obtain 


$$
k=-\frac{2 \mu V_{T} L}{A_{s}\left(p_{T}^{2}-p_{0}^{2}\right)} \frac{d p_{T}}{d t} .
$$

An equivalent form of Eq. (4.5) was derived by Asay et al. (2002) and used by Asay, et al. (2003), Parker et al. (2003), and Asay et al. (2004) to determine the permeability of cooked PBX-9501. It was tacitly assumed in the above derivation that $k$ is a constant; thus, there is a unique functional form for $p_{T}(t)$ that satisfies Eq. (4.5). By integrating Eq. (4.5), with respect to $t$, one obtains

$$
p_{T}(t)=p_{0} \tanh \left[\frac{A_{s} p_{0} k}{2 L \mu V_{T}} t+\operatorname{arctanh}\left(\frac{p_{i}}{p_{0}}\right)\right],
$$

where $p_{i}$ is the initial pressure in the vessel before the valve is opened. It can also be shown that Eq. (4.5) can be written as

$$
k=\frac{2 \mu V_{T} L}{A_{s} p_{0} t} \operatorname{arctanh}\left(\frac{p_{i}-p_{T}}{p_{i} p_{T} / p_{0}-p_{0}}\right) .
$$

Figure 4.3 shows the normalized pressure decay calculated with Eq. (4.6) for a cylindrical porous sample $1 \mathrm{~cm}$ in diameter, $3 \mathrm{~cm}$ long, with a vessel volume of $142.1 \mathrm{~cm}^{3}$. For this calculation, the gas is nitrogen at $186^{\circ} \mathrm{C}$; the sample is also at $186^{\circ} \mathrm{C}$ and has a permeability of $2.3 \times 10^{-16} \mathrm{~m}^{2}$. Notice that $p_{T}(t)$ is necessarily a monotonically decreasing function of time with no inflection points. By taking the second derivative of $p_{T}(t)$, with respect to time, and evaluating at $t=0$, we see that if normal Darcy conditions prevail, $p_{T}(t)$ is always concave upwards at $t=0$ :

$$
\left.\frac{d^{2} p_{T}(t)}{d t^{2}}\right|_{t=0}=\frac{p_{i}}{2}\left(\frac{A_{s} p_{i} k}{L \mu V_{T}}\right)^{2}\left[1-\left(\frac{p_{0}}{p_{i}}\right)^{2}\right] \geq 0
$$

exist. 


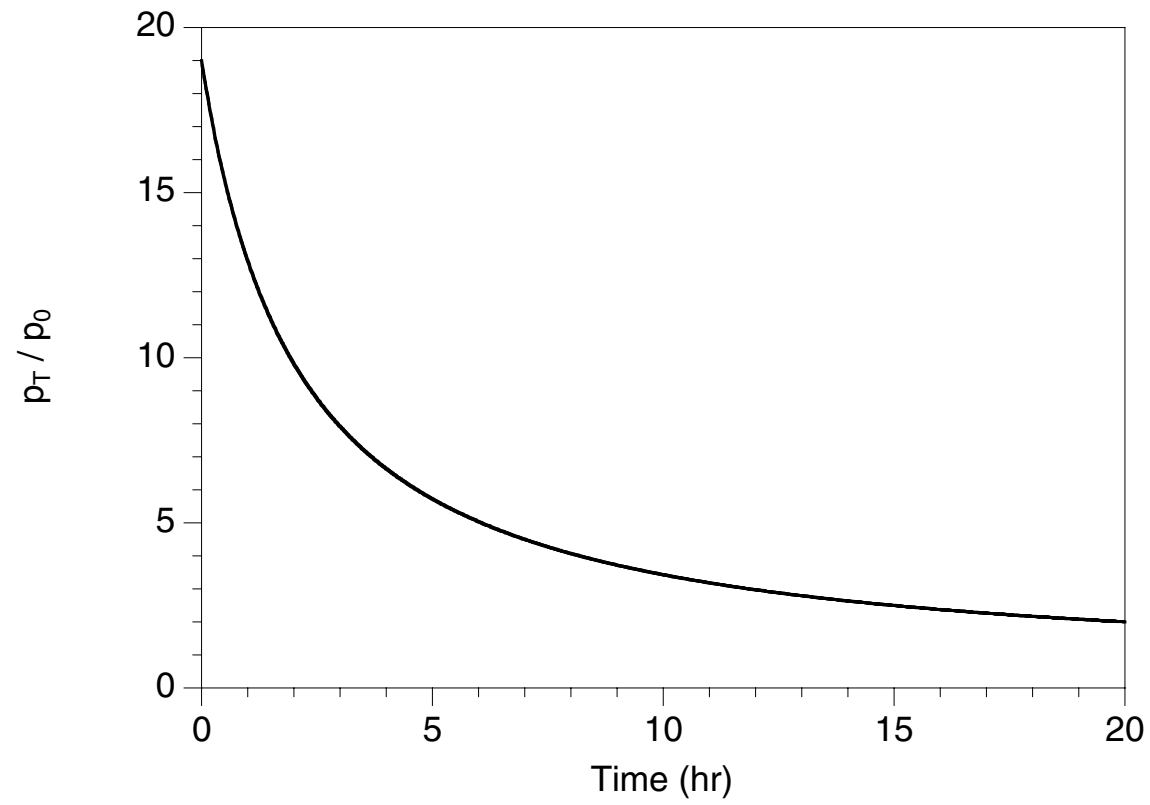

Fig. 4.3. Normalized pressure as a function of time for a single-phase fluid flowing through an isothermal and chemically inert porous sample (normal Darcy conditions).

Although it is simple to operate and use for acquiring data, the gas permeameter design of Fig. 4.2 is not very accurate because it relies on a single measurement, namely, the inlet pressure. The gas flow rate in the sample is inferred from the derivative of the pressure with respect to time. Another drawback of this apparatus is its inability to monitor or measure data at the low-pressure end of the sample. Data from the lowpressure end are important in assessing the possibility of leakage or drainage of the binder. As reported by Asay et al. (2002), the permeability apparatus was susceptible to significant errors. The summary of the results used to calibrate the permeability apparatus is presented in Table 4.2 where the discrepancy is given in terms of the ratio between the reference sample permeability and the measured permeability.

Table 4.2. Comparison between the Permeability Measured by the Sample Provider and the LANL Permeability Apparatus (measurements in $\mathrm{m}^{2}$ )

(Adapted from Asay et al. 2002)

\begin{tabular}{|l|l|l|l|}
\hline Sample & $\begin{array}{l}\text { Sample } \\
\text { Permeability } \\
\text { (as quoted in } \\
\text { Asay et al. 2002) }\end{array}$ & $\begin{array}{l}\text { Permeability } \\
\text { Measured by et al. (2002) } \\
\text { Asay }\end{array}$ & $\begin{array}{l}\text { Measurement } \\
\text { off by a factor } \\
\text { of: }\end{array}$ \\
\hline LA001 & $6.12 \times 10^{-15}$ & $2.09 \times 10^{-15}$ & 2.9 \\
\hline LA002 & $1.01 \times 10^{-14}$ & $4 \times 10^{-15}$ & 2.5 \\
\hline LA003 & $9.53 \times 10^{-16}$ & $2.1 \times 10^{-16}$ & 4.5 \\
\hline LA004 & $1.93 \times 10^{-15}$ & $5.15 \times 10^{-16}$ & 3.7 \\
\hline LA005 & $1.68 \times 10^{-16}$ & $2.1 \times 10^{-17}$ & 8.0 \\
\hline
\end{tabular}




\subsection{Permeability Experiments on Cooked Plastic-Bonded Explosive-9501}

Asay et al. (2004) conducted a series of experiments to measure the permeability of $\mathrm{PBX}-9501$ at $186^{\circ} \mathrm{C}$. They used a variation of the permeameter shown in Fig. 4.2 and heated the HE sample (Fig. 4.4). In one of these experiments, a vessel containing nitrogen gas, at room temperature and an initial pressure of about 20 atm, was connected to a heated sample $\left(186^{\circ} \mathrm{C}\right)$ of potted $\mathrm{HE}$, and the decrease of pressure within the tank was measured. To estimate permeability, they used Eq. (4.5) (Asay et al. 2004). We emphasize that the results reported in their Fig. 2 were based on an equation that is strictly valid for a single-phase fluid flowing through an isothermal and chemically inert sample of a porous material with constant permeability. However, the configuration in Fig. 4.4 is fundamentally different from that treated in Section 4.3 because it departs from normal Darcy conditions in four respects, as we explain below.

First, at $186^{\circ} \mathrm{C}$ the binder is mostly in liquid form, so it fills the interstitial space within the HE. Therefore, heated PBX-9501 is a mixture of molten binder, damaged $\delta$ phase HMX crystals (surrounded by molten binder), and decomposition gases (mostly from the thermal decomposition of BDNPA/F), possibly distributed in the form of bubbles. There could also be gases generated (on the exterior) at the interface between the $\mathrm{HE}$ and the potting material.

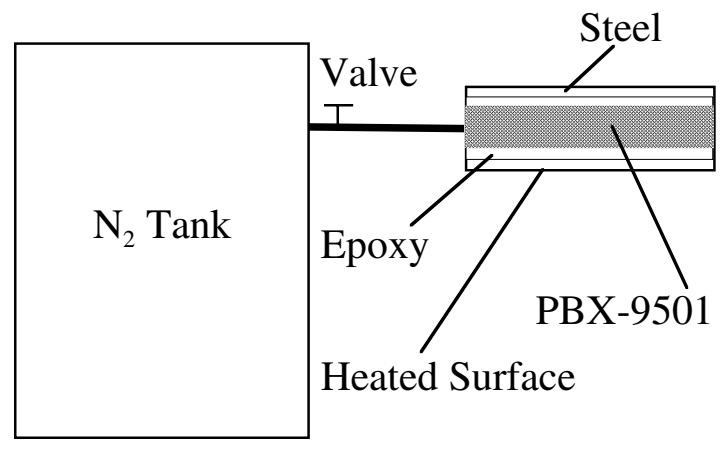

Fig. 4.4. Schematic depiction of the permeameter used by Asay et al. (2004).

Second, during the first three hours of the experiment reported in their paper, the pressure data strongly departs from the behavior predicted by the equation on which they relied to report their results, i.e., Eq. (4.5). For normal Darcy conditions, the pressure is necessarily a monotonically decreasing function of time (see Eq. (4.6) and (4.7)). Figure 4.5 shows their data (Asay et al. 2004) in which the initial decay has downward concavity followed by an inflection point three hours into the experiment. Initially, the nitrogen gas (at $20 \mathrm{~atm}$ ) encounters a medium saturated with a polymeric liquid (molten binder) and, possibly, decomposition gases in the form of bubbles. Therefore, it appears that within the matrix of HMX crystals, there is a nonhomogeneous, two-phase fluid (non-Newtonian fluid and decomposition gases) in contact at one end with a third fluid (the nitrogen gas) from the pressurized vessel. The origin of the concavity change in the pressure history was not discussed in Asay et al. (2004). 
Third, liquid binder drained from the low-pressure end of the cooked PBX-9501 sample at its periphery, as shown in Fig. 4.6. (The picture was taken after the sample had cooled to room temperature.) Notice that the extruded binder accumulated between the epoxy and the sample and that no binder flowed through the center of the sample. This seems to indicate that a preferential path for the drainage of the molten binder was formed at the periphery. In these experiments, the formation of peripheral fissures through which fluid can flow under the action of the pressurized nitrogen can be explained in several ways. For instance, fissures could have formed from a combination of the high pressure (produced by the hydraulic effect of the liquid binder being extruded radially during the $\beta$ to $\delta$ phase transition, in which the HMX crystals expand), the thermal expansion of the binder, and the formation of decomposition gases at the epoxyHE interface. Because the size and characteristics of these fissures would be unique and nonrepeatable, this might explain the lack of qualitative and quantitative repeatability in the data (Fig. 4.7).

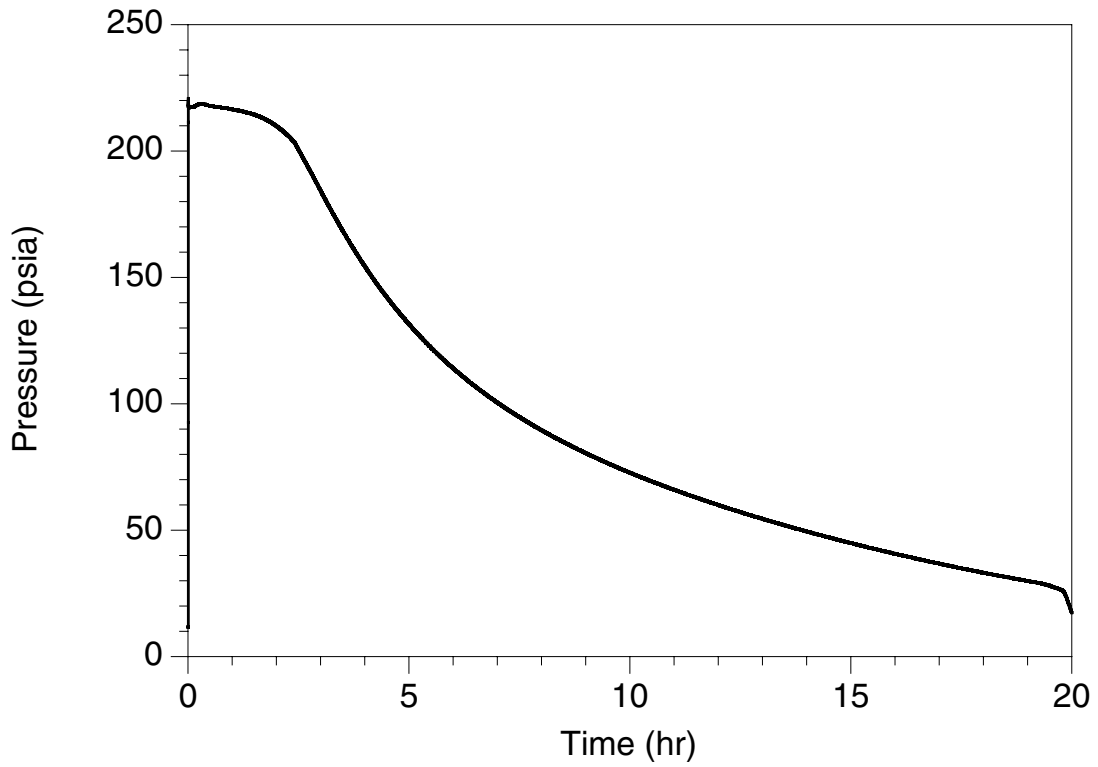

Fig. 4.5. Pressure in the nitrogen tank, as a function of time. (Data provided by $D X-2$. 


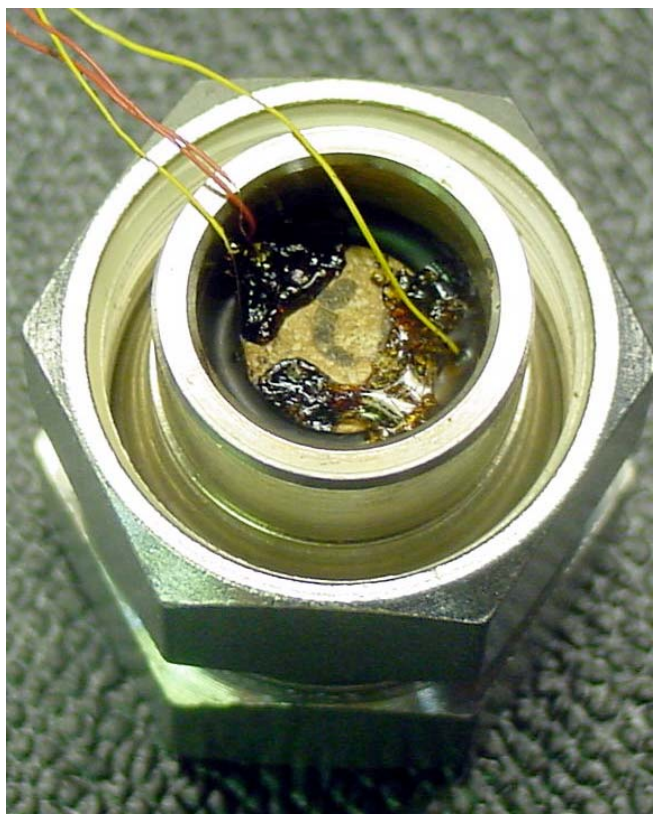

Fig. 4.6. Accumulation of molten binder around the periphery of the low-pressure end of a cooked sample of PBX-9501 after nitrogen gas was fed through it from the other end (Run 12).

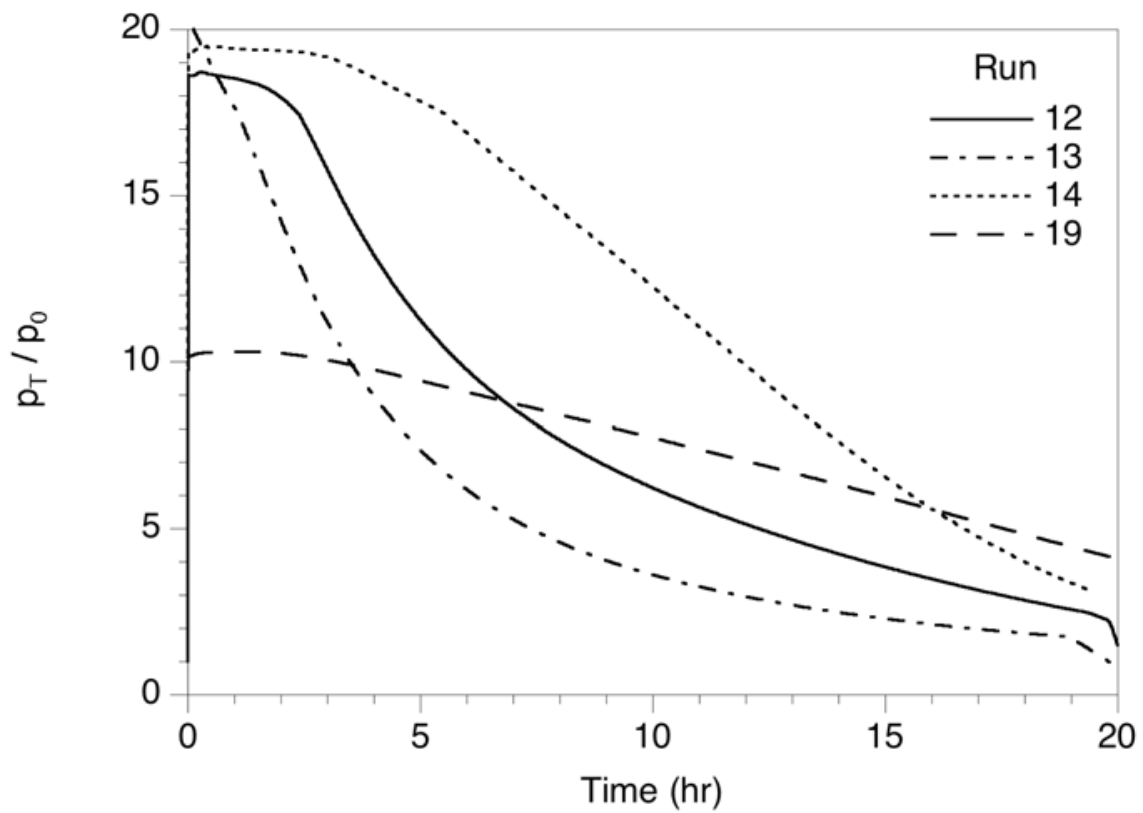

Fig 4.7. Comparison of the pressure histories during different measurements of the permeability of cooked PBX-9501. (Based on data provided by DX-2)

Notice that initially none of the curves in Fig. 4.7 follow the nearly exponential decay shown in Fig. 4.3. Even in Run 13, which seems to follow the decay in Fig. 4.3, there is a 
change in slope after 1 hour. Despite the observable lack of repeatability exhibited by the pressure curves in Fig. 4.7, it has been argued that the tank pressure data are actually repeatable if their temperature histories are taken into account. However, as shown in Fig. 4.8 , the pressure drop in the tank is independent of the sample temperature (the pressure scale in Fig. 4.8 has been reversed for clarity). This is, of course, contrary to what one would surmise by assuming that the pressure drop in the tank was caused by a permeability increase in the sample resulting from a temperature change in the sample. Notice that the most significant pressure decrease (i.e., the earliest decrease) occurs in Run 13 for the "lowest" temperature $\left(184^{\circ} \mathrm{C}\right)$, which is also contrary to what one would expect if the temperature of the sample caused the pressure decrease.

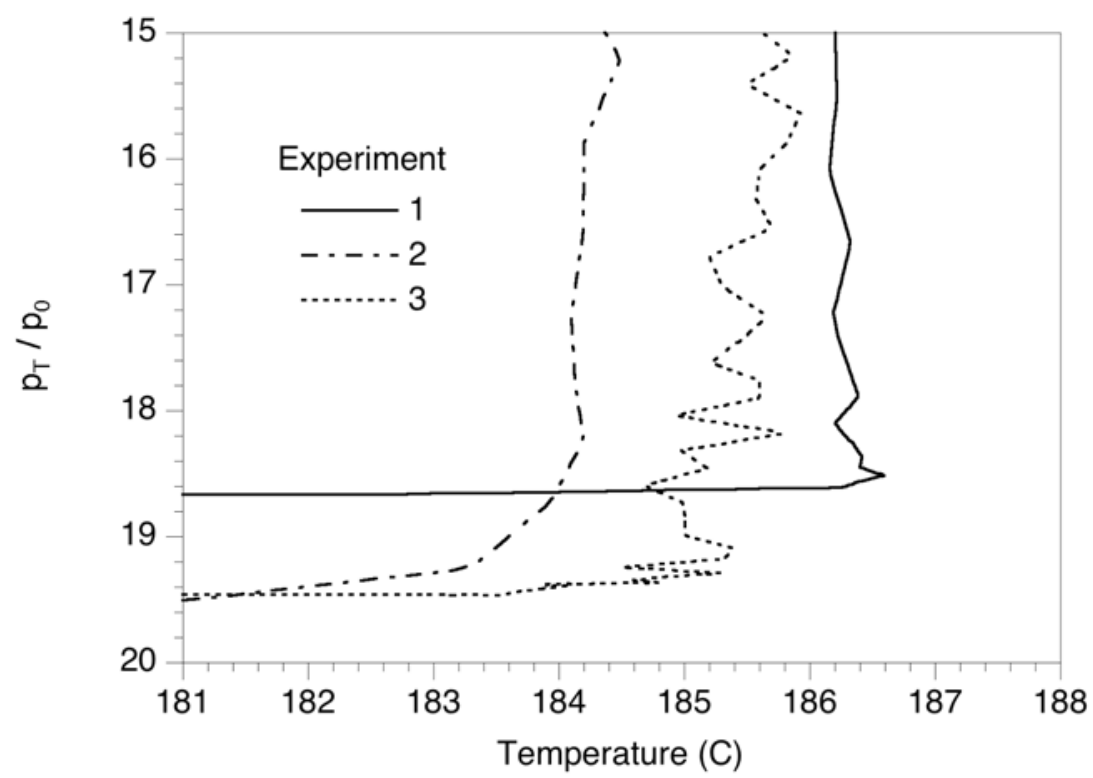

Fig. 4.8. Tank pressure as a function of sample temperature (showing that these quantities are not correlated). (Based on data provided by $D X-2$ )

Fourth, since there is no evidence of a connected network of cracks within cooked PBX-9501, Darcy flow is not valid for the majority of the bulk volume of the sample. A close look at the polarized-light micrographs of thermally damaged PBX-9501, provided in a report by Parker et al. (2003,) does not reveal a porous structure within or outside of the HMX particles. Instead, there appear to be loosely connected cracks that do not bisect HMX particles but rather run along their perimeters. In the confined samples, crack networks appear to develop perpendicular to the direction of the applied pressure gradient and do not provide a direct path from the front to the back of the specimen (Parker et al. 2003). Therefore, the Darcy-flow theory for decomposition gases is not applicable. Furthermore, the epoxy used to stabilize the explosive cannot be differentiated from the cracks or binder in the images, so the regions that have been identified as cracks may be filled with epoxy and/or binder. We are assuming these images would not change significantly if they were recorded at the experiment temperature since the specimen was confined during the thermal transient. 
Using their permeameter, Asay et al. (2004) claimed to have measured the permeability of cooked PBX-9501 by forcing nitrogen gas through the sample. However, the available experimental data suggest that the reported "permeability" was not that of the bulk cooked PBX-9501 to decomposition gases but was instead the "apparent" permeability of the peripheral fissures to nitrogen. There are two phases (liquid binder and gas) flowing axially through this region whose bulk motion could be mistaken as Darcian flow of a single phase through the entire cross section of the sample, since the average flow rate of the nitrogen gas is also directly proportional to the pressure gradient and inversely proportional to the apparent viscosity of the mixture. If a truly porous material is permeable to any fluid (liquid or gas), why did the binder not flow uniformly through the entire cross section of each sample tested? One explanation for this question is that the heated samples were as impermeable as the unheated ones, and heating might have contributed to the formation of fissures through which binder and gases (mostly nitrogen) flowed.

\subsection{Two-Phase Flow through Porous Media}

Before a porous media transport theory for decomposition gases of PBX-9501 can be adopted for simulating pre-ignition, it is essential to determine the rate of binder flow as a function of temperature and to quantify the extent of gas generation and flow caused by thermal decomposition alone. Whether or not there is flow within the HE during preignition that will affect the time-to-ignition, it must first be recognized that when PBX9501 is heated above the thermal decomposition temperature of BDNPA/F, it becomes a multiphase system. Surface tension effects and the nature of the boundaries become important when two immiscible fluids are present in a porous medium. Furthermore, the presence of gas in an initially liquid-saturated, multiphase, porous medium does not necessarily imply that there could be gas flow even in the presence of a pressure gradient (Fetter 1999). A fundamental measurement in two-phase (gas-liquid) flow through porous media experiments is the threshold gas fraction $\phi_{g \text { th }}$ required to establish flow of the gas phase in a porous medium initially saturated with a liquid. The converse is also true; in a porous medium in which the interstitial space is initially filled with gas, there is a threshold liquid binder fraction $\phi_{b t h}$ above which liquid can be transported. Is the conversion of all the BDNPA/F in the binder into gas enough to exceed $\phi_{g \text { th }}$ without having to externally supply a third gaseous component which does not exist in the actual system? If, in fact, it can be established that the gas saturation in the HE is such that $\phi_{g}>\phi_{g t h}$ during the pre-ignition phase, then the relative permeabilities of both binder $k_{r b}$ and gas $k_{r g}$ must be experimentally determined as a function of their volume fractions $\left(\phi_{b}\right.$ and $\phi_{g}$ ) within the $\mathrm{HE}$ and the intrinsic permeability $k_{I}$ as well. These measurements are necessary to specify the flow of gas and liquid in a two-phase system in which two different flow-rate equations are required (Fetter 1999):

$$
u_{b}=-\frac{k_{r b}\left(\phi_{b}\right) k_{I}}{\mu_{b}} \nabla p_{b}, \text { and } \quad u_{g}=-\frac{k_{r g}\left(\phi_{g}\right) k_{I}}{\mu_{g}} \nabla p_{g}
$$


where $u_{i}$ is the velocity of the $i$-th species, $p_{i}$ is pressure, and $\mu_{i}$ is viscosity. To model two-phase flow in a porous medium, five parameters must be experimentally determined:

$\phi_{g t h}, \phi_{b t h}, k_{r g}, k_{r b}$, and $k_{I}$. A better understanding of the actual physical state of cooked PBX-9501 will aid in designing future experiments in which the apparatus is tailored to measure the relevant parameters for model development.

\subsection{Evidence of Binder Melt and Extrusion in Cooked Plastic-Bonded Explosive- 9501}

Tappan et al. (2002) at SNL performed a series of experiments to characterize the mechanical behavior of cooked PBX-9501. Figure 4.9 (from Run 50 of the SNL Hot Cell series) shows the accumulation of binder around the periphery of the upper part of the sample (this picture was taken after the sample cooled to room temperature). In this test, the HE sample fits tightly into a metal cylinder, and the cylinder was heated to $192^{\circ} \mathrm{C}$. The binder closest to the heated cylinder melted and then flowed through the very thin annular space between the HE and the metal (similar to a lubricating flow). Since it appears that a thin annular layer was depleted of some of its binder, this small region could become more permeable to decomposition gases than the rest of the sample. The occurrence of this flow suggests that in the permeability experiments, the molten binder that was extruded out of the HE sample (by the increased volume of the HMX crystals in the $\beta$ to $\delta$ transition, by the thermal expansion of the binder itself, and possibly by decomposition gases) exerted enough pressure between the elastic epoxy layer and the HE sample to create fissures. Therefore, the accumulation of binder seen in Fig. 4.6 could be explained by the flow of molten binder through those fissures and, possibly, through thin regions in the sample near its periphery.

In LSAC Shot 8, binder was found on the recovered end caps and other metal components, indicating that some type of binder flow or extrusion process had developed during the experiment prior to thermal runaway. A density measurement of the residual cooked explosive in Shot 8 would be useful to quantify mass loss, and micrographs might assist with quantifying crack sizes, orientations, and distributions, as well as HMX particle sizes and binder content. Both the micrographs and density measurement could then be compared with those obtained from the permeability experiments. Identifying the role and behavior of the binder is crucial to understanding the PBX-9501 response to cookoff conditions. 


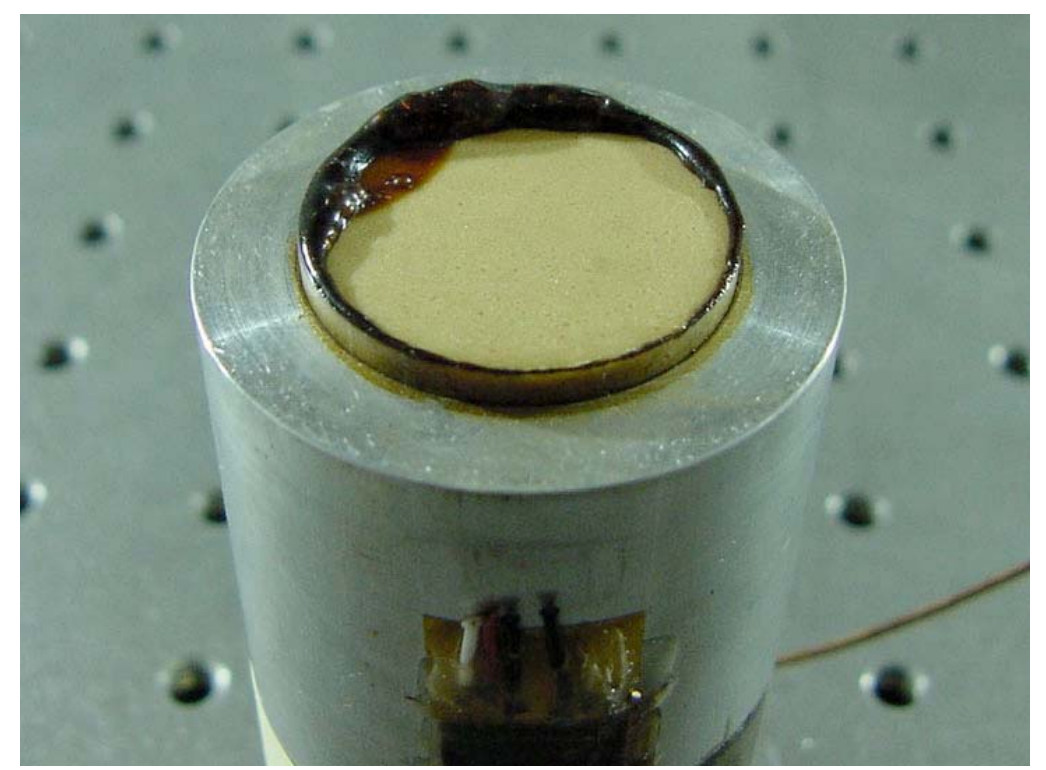

Fig. 4.9. Flow of molten binder through the small annular region between the PBX-9501 and an aluminum cylinder. (Photo provided by SNL.)

\subsection{Effect of Binder Extrusion on the Pressure History}

Because we do not know the effective cross-sectional area of the fissure region (or annular space) between the epoxy boundary or its extent into the HE, the parameters $\phi_{g t h}, \phi_{b t h}, k_{r g}, k_{r b}$, and $k_{I}$, and whether or not there were leaks in the permeability experiments of Asay et al., it would be difficult to develop a robust model that could satisfactorily reproduce all of the data (with a fixed set of transport properties) shown in Fig. 4.7. Some of the uncertainties in characterizing the peripheral fissures and leakage paths that appear to have formed in the samples, together with the fact that their extent cannot be controlled during the experiments, might explain the lack of repeatability in the pressure data shown in this figure. In order to understand what might have occurred in some of the permeability tests, we developed a simplified model to conceptually illustrate that an idealized flow of a liquid phase through a permeable region resembles the earlytime transient behavior in Runs 12 and 14 of Fig. 4.7, namely, the downward concavity of the pressure curve. We emphasize that this is not a two-phase flow-through porousmedia model to simulate drainage but rather a conceptual depiction of a liquid being extruded by a gas phase.

Consider an initially liquid-saturated porous medium region in which one end is connected to a high-pressure gas vessel that drives the flow of the liquid, as shown in Fig. 4.10. We assume there are no direct leakage paths through which gas can escape. Introducing the effect of gas leakage into the model would simply reduce the tank pressure without changing the behavior we want to illustrate. The flow is unidirectional, the velocity profile is uniform (plug flow), and the gas-liquid interface remains planar throughout. For simplicity, the liquid is assumed to be Newtonian, although in reality, polymeric liquids - because of their high molecular weight-are non-Newtonian (strainrate dependent). Since the viscosity of a polymer melt is at least more than two orders of magnitude larger than that of a gas, the viscosity of the liquid phase will dominate and 
thus determine the flow rate. Using the same nomenclature defined in Section 4.3, the rate of change of the gas/liquid interface is

$$
\frac{d z(t)}{d t}=\frac{k\left[p_{T}(t)-p_{0}\right]}{\mu_{l}[L-z(t)]}
$$

where $z(t)$ is the location of the gas/liquid interface, and $\mu_{l}$ is the viscosity of the liquid.

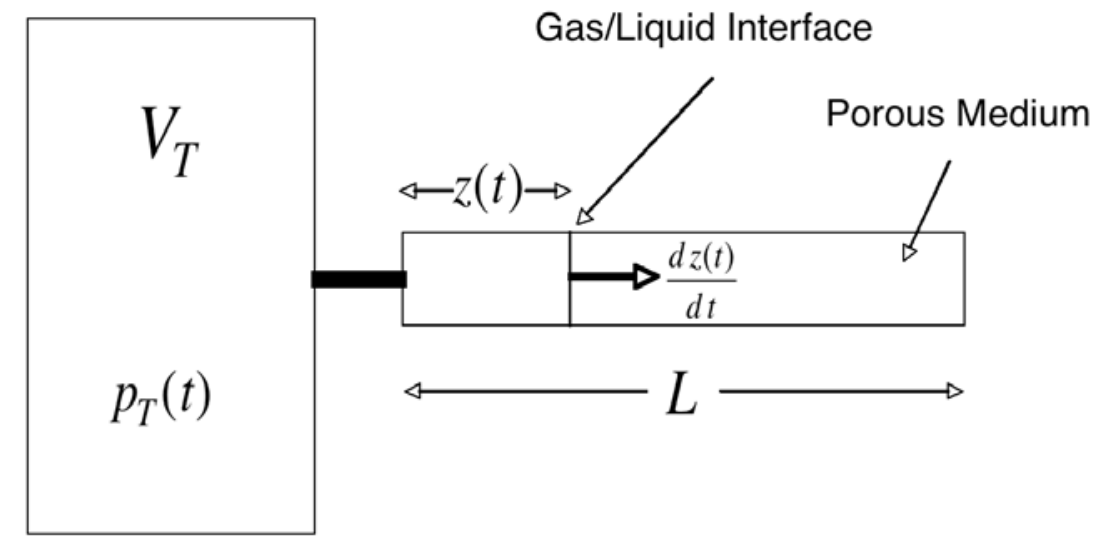

Fig. 4.10. Extrusion of a liquid through a porous medium by a gas insert.

Conservation of mass requires that the rate of change of the gas density $\rho_{g}$ within the vessel must balance the net rate of gas flux entering the porous medium. Because the gas flow rate into the sample is determined by the motion of the gas/fluid interface,

$$
V_{T} \frac{d \rho_{g}(t)}{d t}+\rho_{g}(t) A_{s} \frac{d z(t)}{d t}=0
$$

In this situation, the gas flow into the sample takes place at a much lower rate than it would if the sample were not saturated with a liquid. Therefore, the pressure within the vessel will be essentially the same as the back-pressure driving the gas/liquid interface. If we use the ideal gas law and assume that $V_{T}$ is much greater than the interstitial volume of the sample and the conduit between the tank and the sample, Eq. (4.10) becomes

$$
\frac{d p_{T}(t)}{d t}+\frac{A_{s} k}{V_{T} \mu_{l}} \frac{p_{T}(t)\left(p_{T}(t)-p_{0}\right)}{L-z(t)}=0
$$

The idealized behavior of a liquid being extruded through a porous material by a gas is described by Eqs. (4.9) and (4.11) for the dependent variables $z(t)$ and $p_{T}(t)$. The initial conditions are $z(0)=0$ and $p_{T}(0)=p_{i}$. We will calculate the pressure in 
dimensionless form to illustrate the pressure behavior of the system. By introducing the following dimensionless variables $x=z / L, \quad \tau=k A_{s} p_{i} t /\left(\mu L V_{T}\right), \quad \gamma=A_{s} L / V_{T}$, $P=p_{T} / p_{i}$, and $\varepsilon=p_{0} / p_{i}$, the system of equations becomes

$$
\left\{\begin{array}{c}
\frac{d P(\tau)}{d \tau}+\frac{P(\tau)(P(\tau)-\varepsilon)}{1-x(\tau)}=0 \\
\gamma \frac{d x(\tau)}{d \tau}-\frac{P(\tau)-\varepsilon}{1-x(\tau)}=0
\end{array},\right.
$$

with initial conditions $P(0)=1$ and $x(0)=0$, which is solved numerically.

Figure 4.11 shows the solution of Eqs. (4.12) up to the point where all of the liquid has been flushed from the porous sample by the gas. Notice that the pressure curve has a downward concavity, which is reminiscent to that found in Runs 12 and 14, shown in Fig. 4.7.

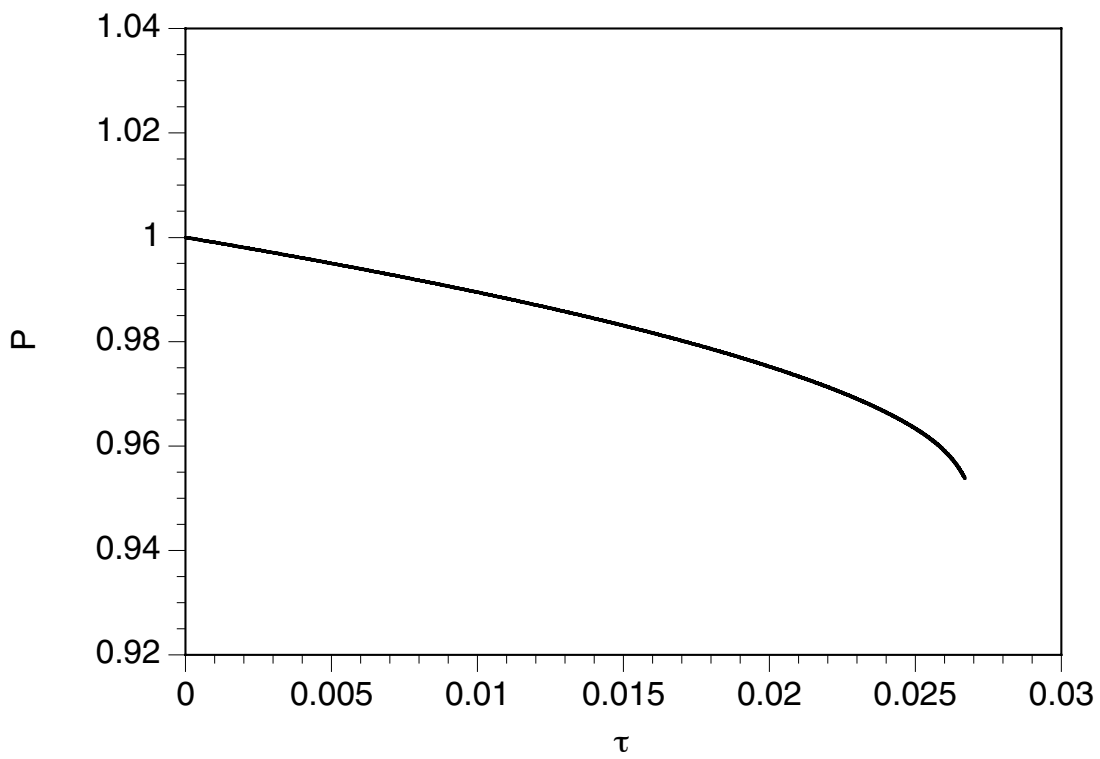

Figure 4.11. Dimensionless pressure versus time for a model in which liquid is flushed out of a porous sample by a gas.

In Fig. 4.12, the solution of Eq. (4.12) has been matched to the dimensionless form of Eq. (4.6). The initial pressure transient and the inflection point near the time when all of the liquid has been purged from the system are consistent with the behavior shown in Fig. 4.5. If gas leaks were included in the model, the pressure would drop further during the extrusion of liquid. In reality, we expect the process to be considerably more complex than this idealized description of extrusion where there could be other phenomena not yet identified. However, this simple model offers some insight into an experimental observation that has not received much attention. 


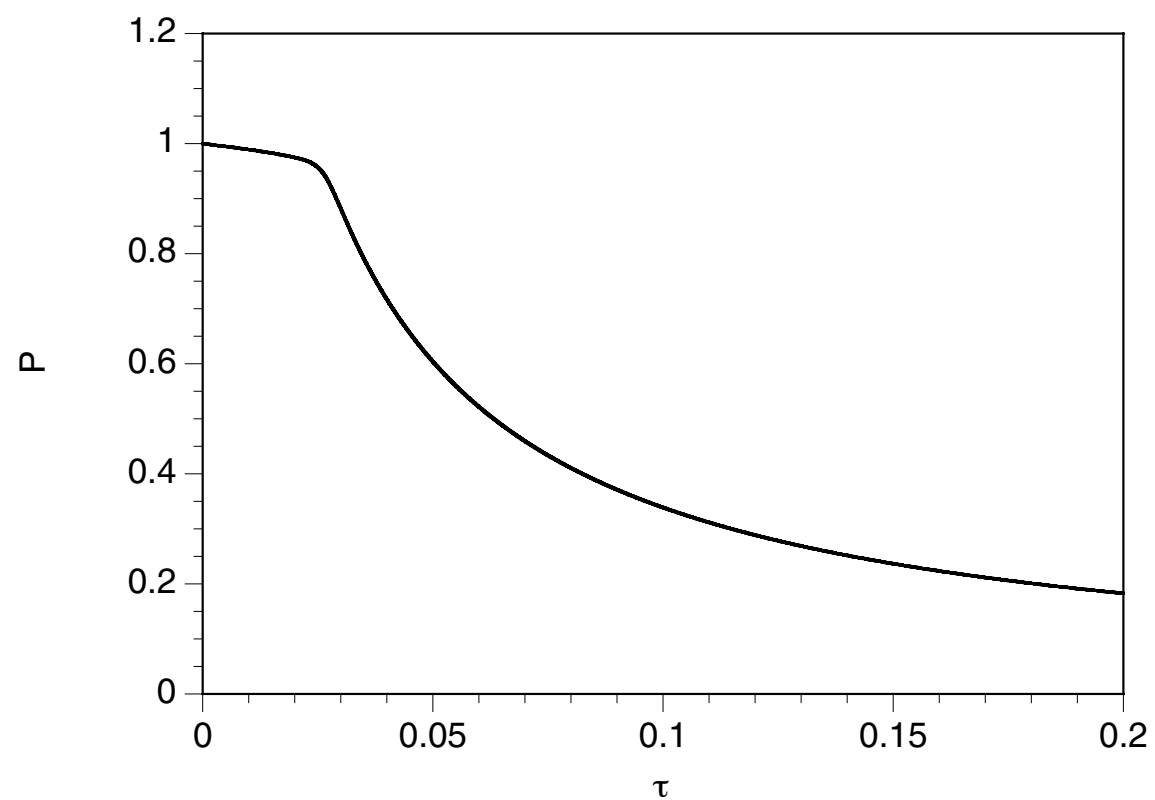

Fig. 4.12. Dimensionless pressure versus time for a model during and after a hypothetical flushing of a liquid out of a porous sample (by a gas).

\subsection{Modeling Based on the Darcy-Flow Hypothesis}

The origin of the idea of pre-ignition modeling based on the Darcy flow hypothesis can be traced to an early interpretation in 2000 of the photographs of the inner-liner collapse in Shot 3. This interpretation, based on the supposition that all of the dark areas in the filed of view of the camera were metal (a discussion of this issue can be found in Section 3.2; see also Figs. 3.6, 3.9, and 3.10), led to the statement, "The combination of the gradually increasing collapse speed and the high degree of symmetry suggest a mechanism where a large fraction of the explosive is ignited before much of it can react" (Howe et al. 2000b). It was hypothesized that in order for PBX-9501 to ignite before reacting, the decomposition gases had to permeate throughout the HE, which was regarded as a porous medium where gas flow would be governed by Darcy's law.

At LANL, D-5 (Nuclear Design and Risk Analysis) from the Decision Applications Division has been conducting pre-ignition simulations on at least one of the shots from the LSAC series. Zerkle and Luck (2003) developed a computer model in which the HE is treated as a porous matrix where the volume-averaged velocity can be described by the Darcy flow equation for a single phase. In their model, they assumed that the gas produced during the thermal decomposition of the HE permeates through a hypothetical matrix of HMX crystals devoid of molten binder. Using the four-step chemical kinetic model developed by Dickson et al. (2000) (see also the Appendix I in Aviles-Ramos 2004), Zerkle and Luck carried out two-dimensional simulations (over the midplane of the assembly) in which the gas flow was governed by Darcy's law and the permeability was calculated from the Blake-Kozeny equation (Bird et al. 1960) for a packed bed of spherical particles of identical size. It is not clear from the paper of Zerkle and Luck 2003 what specific boundary conditions and what characteristic particle size were used in their computations. Their model results were compared with the experimentally recorded time to ignition for Shot 3 . They predicted that the time to ignition occurred at $37659 \mathrm{~s}$ (Zerkle 
2003), which is within $0.05 \%$ of the experimental value of 37676 s (Zerkle 2003). Since this minute departure from the data is less than any of the known individual uncertainties in the experiments, the excellent agreement may in part be attributed to parameter tuning of experimentally undetermined variables. In Dickson's kinetics parameters, which were calibrated for the radial cookoff tests and not for the LSAC, there is an error between the simulated and experimental temperature profile (1\% for fast heating and about $7 \%$ for slow heating) and the time to ignition (about 0.6\%) (see Appendix I in Aviles-Ramos 2004).

There is a chain of experimental uncertainties that must be recognized: namely, the lack of generality of the reaction kinetics; the uncertainty in the thermophysical properties of reactants, intermediates, and products; the lack of measurements on the thermal contact resistances between the cooked HE and the metal assemblies (these have to be estimated in numerical simulations); the uncertainty in quantifying confinement; our lack of knowledge about the microstructural changes within the HE matrix and their effects on the time-to-ignition; and the lack of conclusive evidence that Darcian gas flow actually takes place throughout the HE. In the LSAC experiments, some variables-such as the tolerances between the $\mathrm{HE}$ and the assemblies and the degree of confinementwere not reported and apparently were not controlled. The uncertainties associated with uncontrolled parameters become evident in the measured time-to-ignition for Shots 5 and 6, where they differed by $28 \%$ (Fig. 4.13). These two shots had the same casing material (but probably not the same degree of confinement because apparently the machining tolerances were different for copper and brass) and were subjected to similar thermal profiles. In light of these facts, one cannot expect cookoff simulations to be as good as the experiments.

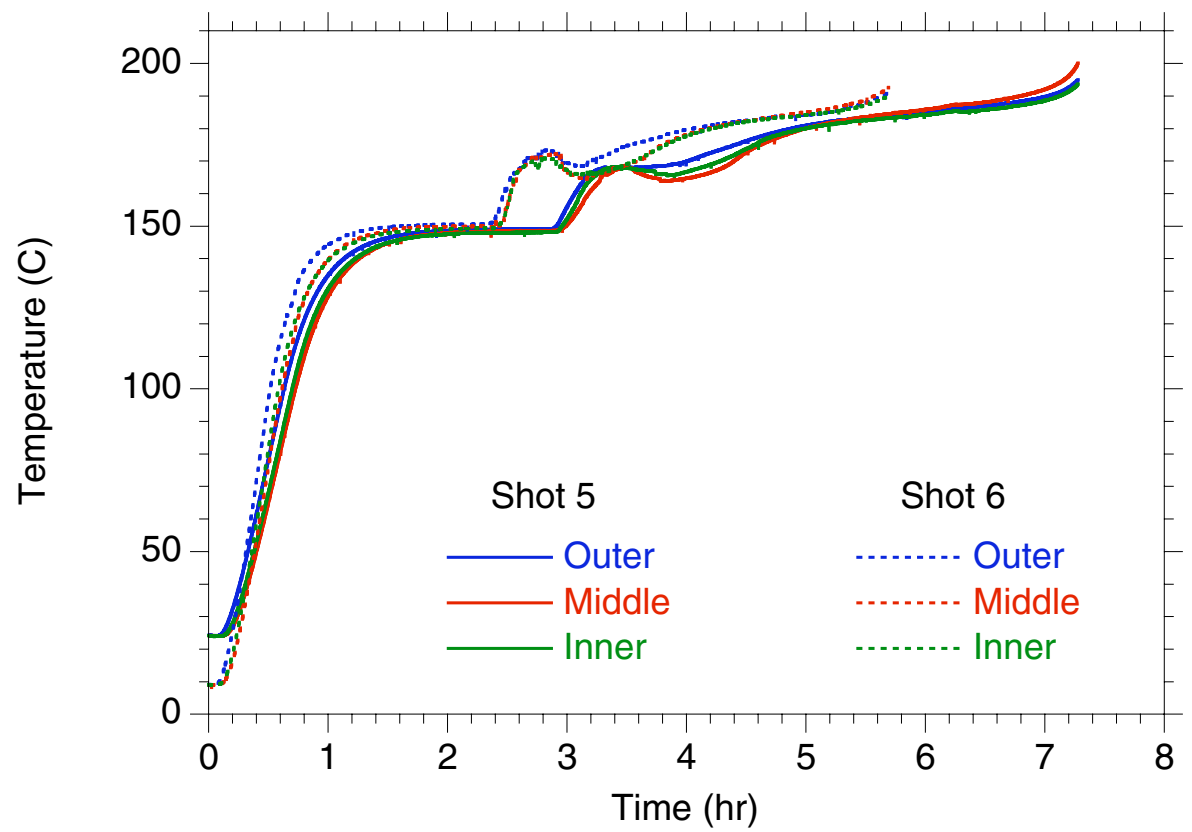

Fig. 4.13. Comparison of averaged thermocouple measurements for the inner, middle, and outer explosive regions for LSAC Shots 5 and 6 (copper liners). (Based on data provided by DX-2) 
Zerkle and Luck (2003) also relied on the location of the ignition point to compare with their simulations. However, there are not enough data points from the experiments to accurately pinpoint the exact location at which the highest temperature actually occurred (there are only three thermocouples in the radial direction, placed every $60^{\circ}$ ). In addition, the response of thermocouples is at best on the order of tens of milliseconds, and before ignition, the temperature field could be very different in the vicinity of the ignition point from the last instant recorded experimentally. There is also an uncertainty in the location of the thermocouples, and with only 18 data points in the annular region of the HE, it is not possible to construct a temperature contour map and obtain the degree of accuracy shown in the numerical simulations. Even if the azimuthal location of the highest temperature coincided, by chance, with that of three fast thermocouples, it would be difficult to fit a nonmonotonic radial temperature profile to accurately determine the maximum of the function and its location (knowing that there is an uncertainty associated with the radial and angular location of the thermocouples).

As shown in the previous sections of this chapter, the permeability experiments have not conclusively proven that there is a flow of decomposition gases which are driven by pressure gradients that originated from temperature gradients. Even if there were localized transport of gas within the HE, one cannot infer that such gas motion is governed by Darcy's law for single-phase fluids. In permeability experiments, the drainage of liquid binder that occurred mostly from the periphery of the sample and was propelled by a pressurized inert gas does not support the hypothesis that PBX-9501 becomes fully permeable when heated to temperatures within the cookoff range. More likely, these experiments indicate that bulk samples heated to $185^{\circ} \mathrm{C}$ remained practically impermeable or even as impermeable as they were at room temperature.

Dickson's kinetic mechanism (without any modifications) has been assessed for the SITI experiments at SNL, where the explosive mass, heating rate, and confinement are different from those in the LSAC experiments. Kaneshige et al. (2002) applied Dickson's kinetics to the SITI experiments and concluded that the model overpredicts "time to ignition significantly over the full temperature range investigated." This result is not surprising because as Dickson et al. (see Appendix I in Aviles-Ramos 2004) showed, a model based on reaction kinetics parameters fitted to a particular heating rate does not generally reproduce the thermal behavior and time to ignition for a different rate. More importantly, some of the fitted kinetics parameters will vary with the degree of confinement, which substantially affects the time-to-ignition. Figure 4.14 shows a comparison of the time to ignition data (spanning the cookoff temperature range from $180^{\circ} \mathrm{C}$ to $200^{\circ} \mathrm{C}$ ) between the SITI and DX-2 experiments (see Appendix I in AvilesRamos 2004). The longer times to ignition in the DX-2 tests indicate that confinement was not as carefully controlled as in the SITI experiments. Compared with the SITI data, the time at point 1 in Fig. 4.14 is off by 36\%, and the time at point 2 is off by a factor of 2.8. The reaction kinetics used by Aviles-Ramos (2004) and Zerkle and Luck (2003) was calibrated to the test represented by point 1 in Fig. 4.14. In this test, the sample was heated along a linear temperature ramp, from room temperature to $190^{\circ} \mathrm{C}$ in 0.56 hour. As shown in Chapters 2 and 3, the LSAC assemblies were heated along a path completely different from the one used to calibrate the reaction kinetics developed by Dickson et al. 


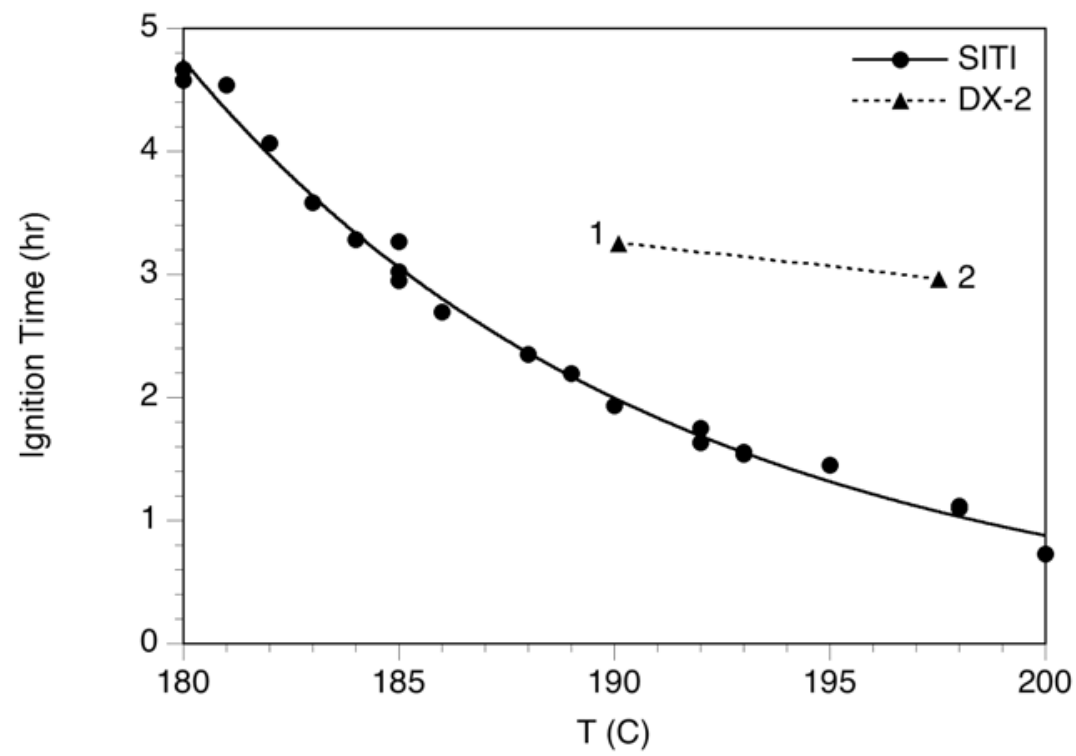

Fig. 4.14. Time to ignition data within the cookoff temperature range. (Based on data provided by SNL and DX-2)

Even if it were assumed that gas was uniformly generated within the HE and the molten binder did not exist, the measured temperature gradients in shots other than Shot 3 would not be high enough to produce high-pressure gradients that could transport the gas through the interstitial space far enough to have a substantial effect on the temperature distribution. Therefore, including Darcian gas transport (through a hypothetical bed of HMX particles that contain no molten binder) in pre-ignition simulations might unnecessarily increase the complexity of the governing equations without significantly increasing the accuracy of predicting the time to ignition.

In some of the LSAC tests, the explosive was more or less uniformly heated (until near thermal runaway), which resulted in small temperature gradients and, correspondingly, only small pressure gradients. Figure 4.15 compares the averaged (azimuthally at a fixed radial location) explosive thermocouple reading in the inner, middle, and outer regions for Shots 3 and 6. A small, averaged temperature gradient was recorded in Shot 6, and a larger gradient was measured in Shot 3. In Shot 6, just before thermal runaway, there were temperature gradients that would produce larger pressure gradients than those produced at earlier times, and very localized gas flow could occur. However, this condition occurred at the end of the thermal transient, and it was concentrated in a small region around the runaway zone; thus, there is little time for the spread of gases to dominate the overall temperature distribution before runaway. In Shot 3 , the pressure gradients may be larger, but it is questionable whether they are sufficient to produce significant gas flow. The issue of whether gas generation can produce pressure gradients within a decomposing mixture of HMX and molten binder certainly requires further investigation. It could be ascertained by conducting a small-scale experiment in which a temperature gradient (representative of the LSAC gradients) is established within a cylindrically confined PBX-9501 sample of sufficient length to establish the condition 
and mobility of the binder and to determine the motion (spatial and temporal) of product gases.

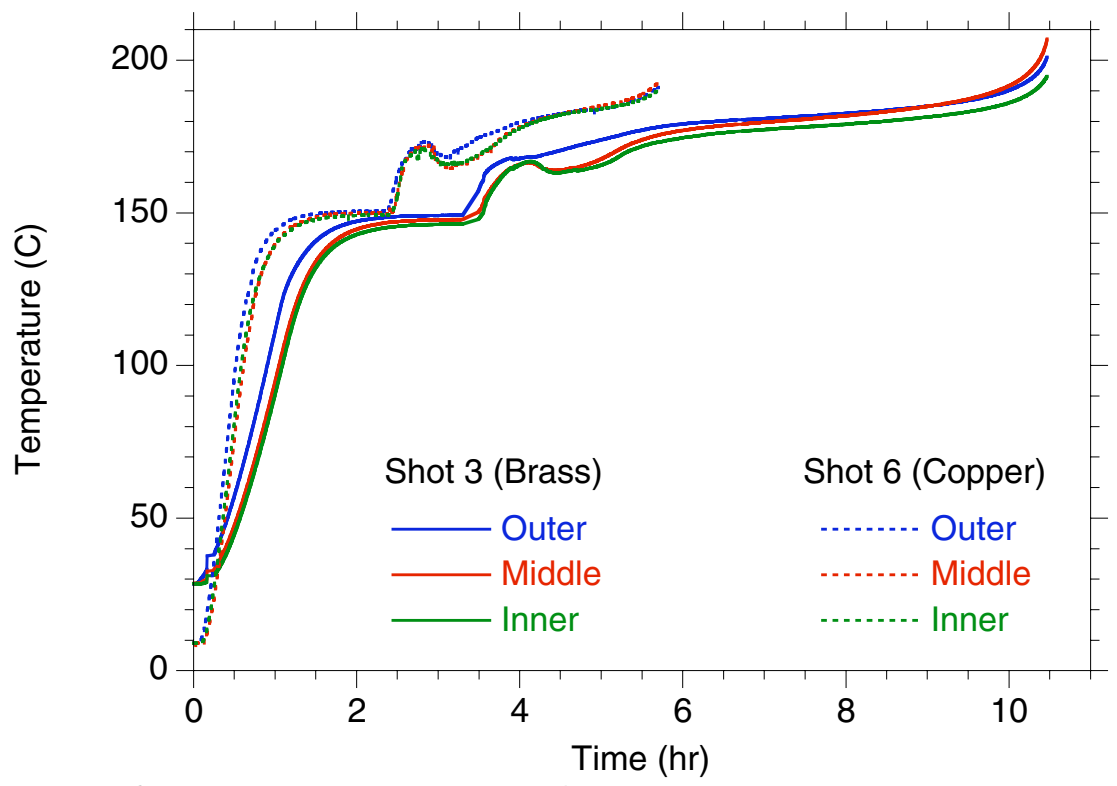

Fig. 4.15. Comparison of averaged (azimuthally at a fixed radial location) thermocouple measurements for the inner, middle, and outer explosive regions for LSAC Shots 3 and 6. (Based on data provided by DX-2)

\subsection{Predictive Capability and Limitations}

Model evaluation and validation are iterative processes whose ultimate goal is the prediction, usually by numerical simulations, of coupled phenomena occurring under limited regimes and constraints. A truly predictive numerical capability must consist of mathematical models capable of consistently being in agreement, within an acceptable margin of error with a variety of experiments. If the problem to be simulated involves a combination of phenomena that are not well understood and for which only crude mathematical representations are available, then the acceptable error margin must be at least as large as the combined uncertainties of the models in predicting each individual phenomenon. In general, simulations of complex phenomena cannot be better or even as good as the experiment. Furthermore, regardless of the agreement, comparing the simulation results with a single experiment is by no means sufficient to establish that the models could adequately describe cookoff phenomena in general.

Ultimately, we are interested in developing a predictive capability for assessing the dynamic loading effects of a thermally driven explosion of PBX-9501 on an adjacent metal assembly. The first step toward achieving this goal is to develop a reaction-kinetics mechanism and a heat-transfer modeling capability that are technically defensible and can predict the time-to-ignition trends that were measured in cookoff experiments. Currently, we have adequate tools for heat transfer analysis, but we do not have a preignition predictive capability yet. We are using the kinetics developed by Dickson et al. (1999, 2000) and by Tarver and Tran (2004) and can predict, a posteriori, the time-toignition in the LSAC geometry by enforcing estimated boundary conditions and/or resorting to modification of the kinetic parameters. 
Currently, available global reaction kinetics models are essentially curve fits that are tailored for certain kinds of geometries and specific heating rate conditions and are not necessarily general or self-consistent. For instance, the scheme proposed by Tarver and Tran (2004) for the thermal decomposition of PBX-9501 assumes that the HMX reaction is independent of the binder kinetics. It appears that the binder plays a role on the ignition of PBX-9501 during cookoff as suggested by Kaneshige et al. (2003) and from recent unpublished experiments by Behrens.

Using the finite element code CALORE, Aviles-Ramos (2004) developed a methodology for the validation of reaction kinetics models. His modeling approach is based on the purely conductive mechanisms of heat transfer within the HE and the metal assembly. Relying on the LSAC thermocouple data from the HE and the assembly to establish the boundary conditions for his simulations, he first optimized three parameters in Dickson's kinetic scheme and then adjusted the thermal conductance to match the times to ignition for Shot 3 and Shots 5 through 8. In order to match the thermocouple readings within the $\mathrm{HE}$, Aviles-Ramos suggests that in some instances, the thermocouple data from the metal assembly may have to be modified before they are used as boundary conditions. To illustrate just how far we are from a truly predictive capability for preignition, Aviles-Ramos had to modify the boundary conditions and the thermal and kinetic parameters in order to match the simulated times-to-ignition to the thermocouple data. However, Aviles-Ramos' simulations also matched the LSAC data without invoking the Darcy flow hypothesis.

It is clear that there are many gaps in the data that must be resolved in order to make accurate predictions. For instance, there are no data for the thermal contact resistance, and this precludes accurate estimates of the thermal behavior. To compensate, preignition modelers have applied arbitrary spatial and temporal thermal-contact resistances to match the data.

Because there are too many unknowns in the LSAC experiments, it is not realistic to expect that existing models can predict times-to-ignition within accuracy of less than $1 \%$. A more reasonable estimate would be one within the experimental margins of error for the measured times-to-ignition, derived from experiments that used similar confinement geometry and similar heating profiles. In the LSAC experiments, the measured time-toignition for configurations that had the same confinement material and were subjected to similar thermal profiles could vary by $\pm 28 \%$ (Fig. 4.13 ). 


\section{Baseline Simulations: Detonation of the Pristine Plastic- Bonded Explosive-9501}

We performed a series of detonation simulations with the LSAC geometry in order to establish a baseline with which we could compare the dynamical deformation of a metal when it is subjected to a thermally initiated explosion. For an LSAC assembly detonated with pristine PBX-9501, two cases were simulated. In the first case, detonation was initiated at a single point located in the midplane of the HE, perpendicular to the axis of symmetry for the assembly, near the outer liner, and at the metal-HE interface (see Fig. 5.1). In the second case, detonation was simultaneously initiated at two points within the $\mathrm{HE}$ in the upper and lower parts of the assembly. The line joining these points was parallel to the axis of symmetry (see Fig. 5.1).

In our computational model, the end caps are fused to the liners so that the casing is in one piece, whereas in the experiments, the end caps and liners were joined as a threaded assembly. In most of the simulations, a computational domain of $0.56 \times 10^{6}$ cubic cells was used, which corresponds to a cell dimension of $0.2 \mathrm{~cm}$ and a quarter of the entire LSAC assembly. In some instances such as in Fig 5.1 where the detonation points are not on the midplane, a half model was required. Convergence of the numerical solution was checked by halving the cell size $(0.1 \mathrm{~cm})$ and increasing the number of cells to $4.5 \times 10^{6}$. The global evolution of the deforming assembly was indistinguishable between simulations using the refined $4.5 \times 10^{6}$ cell and the $0.56 \times 10^{6}$-cell models. However, noticeable differences were found on a point-by-point basis for the pressure, density, and internal energy within the inner liner. These differences are attributed to the smearing of the shock wave across three material cells along the radial direction within the inner liner in the $0.56 \times 10^{6}$-cell model, since at least six cells are needed to capture the shock propagation through a metal layer.

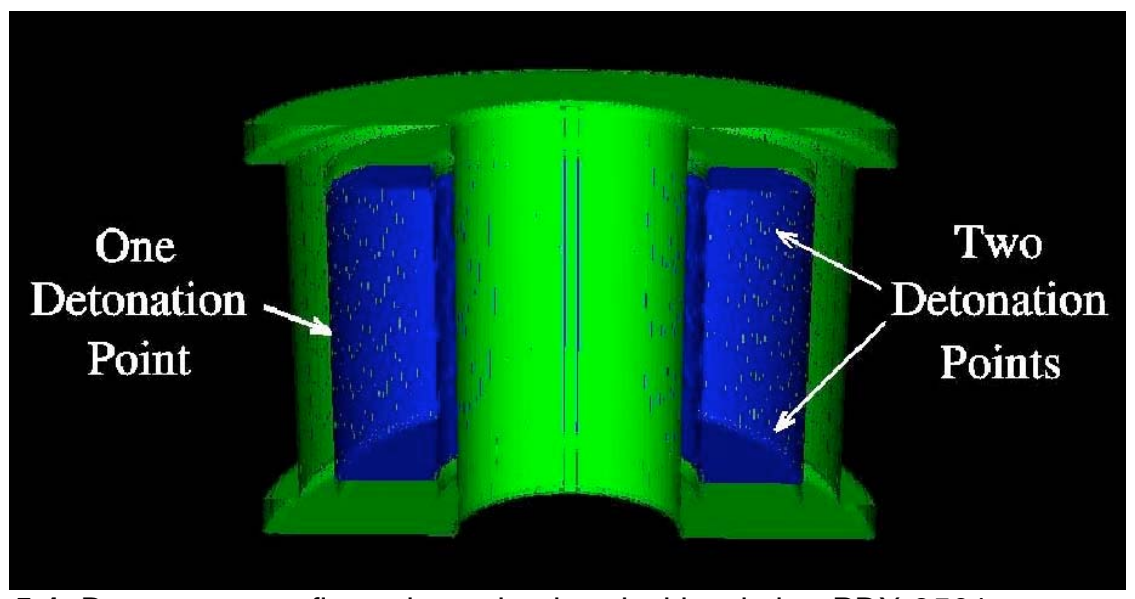

Fig. 5.1. Base-case configurations simulated with pristine PBX-9501.

Casing expansion and inner-liner collapse were qualitatively and quantitatively very similar during the first $25 \mu \mathrm{s}$ in both the one- and two-point detonations. Figure 5.2 shows the velocity magnitude mapped onto the deforming copper assembly at $25 \mu$ s for 
both detonation configurations. The average expansion rate of the outer liner agreed with the velocity obtained from Gurney's model (Cooper 1996). For PBX-9501, the Gurney velocity coefficient is $0.29 \mathrm{~cm} / \mu \mathrm{s}$ (Explosives Reference Guide 2004), and the calculated velocities of the fragments for the LSAC lie somewhere between those computed for the cylinder and sandwich configurations:

$$
\begin{aligned}
& V_{\text {outer }}=0.09-0.12 \mathrm{~cm} / \mu \mathrm{s} \\
& V_{\text {inner }}=0.25-0.27 \mathrm{~cm} / \mu \mathrm{s} .
\end{aligned}
$$

After $30 \mu$ s, the inner liner in the two-point detonation collapsed at a slightly higher average rate than did the inner liner in the one-point detonation case, as shown in Fig. 5.3. In each case, the liner collapse is not azimuthally or axially symmetric, as evidenced by the computed velocity field at the inner liner. Material elements on the inner liner closest to the detonation points move faster than those farther away, as shown in Figs. 5.2 and 5.3. Therefore, in both cases, the geometry of the collapsing inner liner is not perfectly symmetric with respect to the original axis of the LSAC assembly.
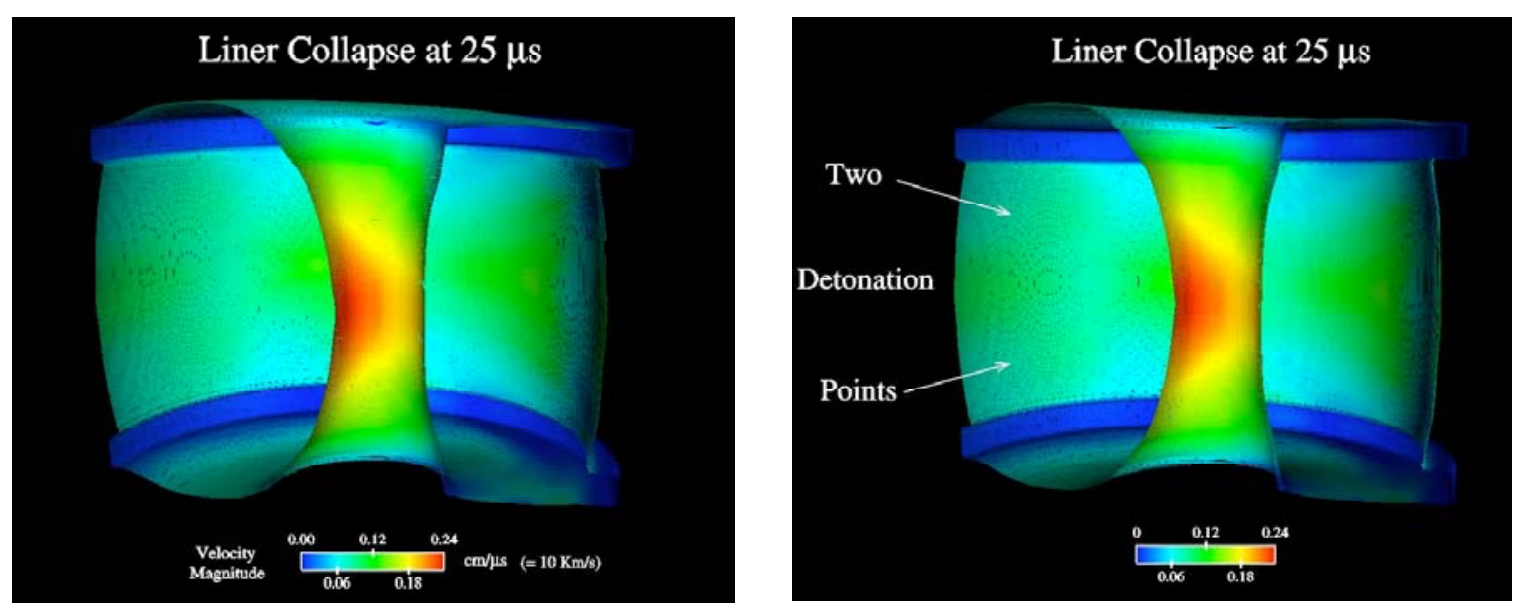

Fig. 5.2. At $25 \mu \mathrm{s}$, the velocity magnitude is shown for a one-point (left) and twopoint (right) detonation of the LSAC assembly with pristine PBX-9501. 

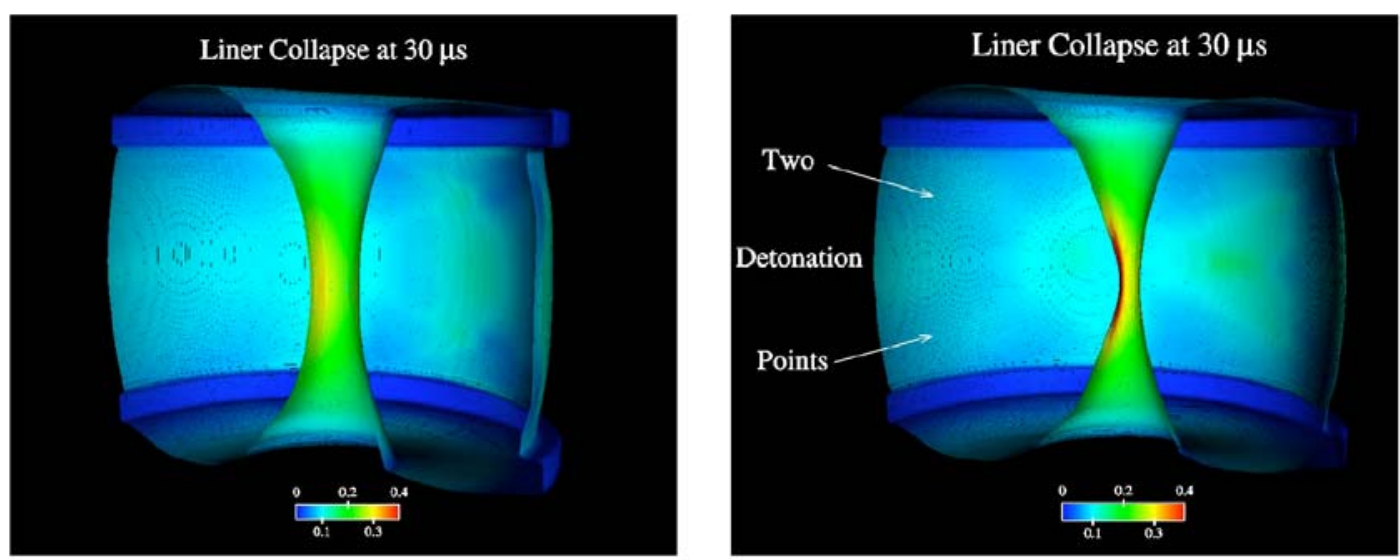

Fig. 5.3. At $30 \mu \mathrm{s}$, the velocity magnitude is shown for one-point (left) and twopoint (right) detonation of the LSAC assembly with pristine PBX-9501.

\subsection{Large-Scale Annular Cookoff Assembly Fracture/Failure}

For the one-point detonation, an assessment of the fracture and, ultimately, the fragmentation process for the outer liner and end caps of the LSAC assembly can be made by analyzing the effective plastic-strain-rate field (see Fig. 5.4). During the first 25 $\mu$ s, the highest strain rates were on the inner part of the assembly's upper and lower end caps. Eight microseconds after detonation, a strain-rate front was formed on the end caps around the junctions at the inner and outer liners and in the vicinity of the detonation point. This wave front bifurcated into two wave fronts that propagated in opposite directions around each end cap. At about $25 \mu \mathrm{s}$, the fronts collided, after which time the highest strain rates occurred on the collapsing inner liner. The location and magnitude of the strain rate indicate that fracture might have taken place initially around the end caps. Currently, PAGOSA does not have a fracture and fragmentation model that can directly predict fragment sizes and distributions. However, fragment sizes could be estimated by applying the Grady-Kipp model (Grady and Kipp 1985a, 1985b), since PAGOSA can compute the strain-rate field. 


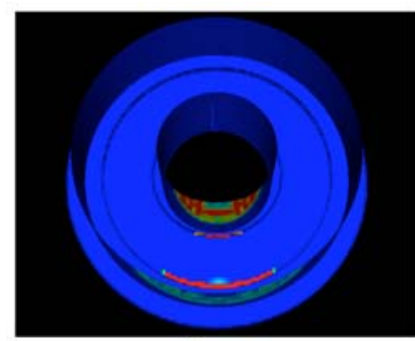

$8 \mu \mathrm{s}$

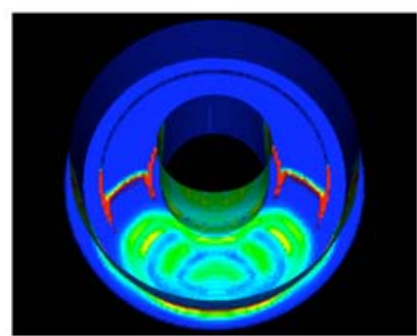

$15 \mu \mathrm{s}$

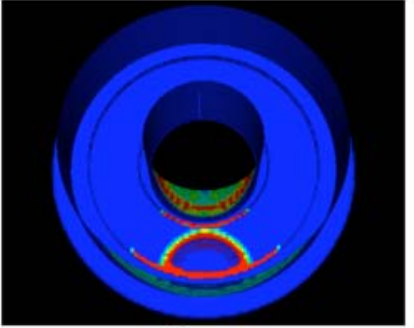

$9 \mu \mathrm{s}$

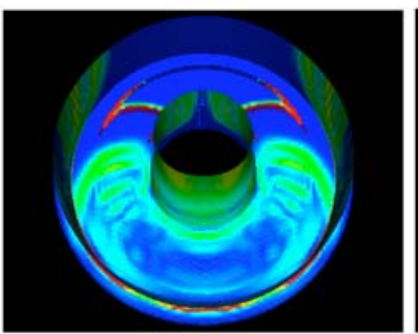

$20 \mu \mathrm{s}$

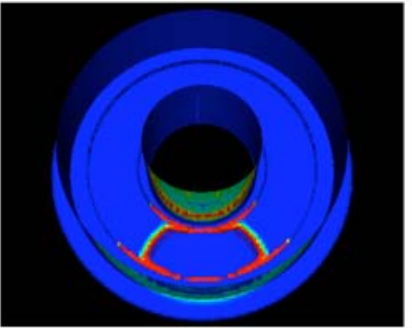

$10 \mu \mathrm{s}$

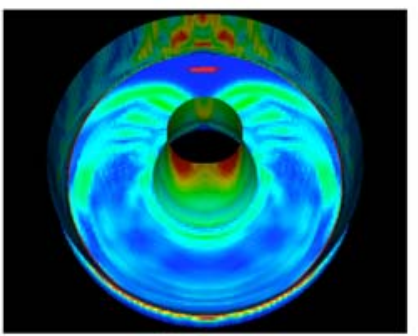

$25 \mu \mathrm{s}$

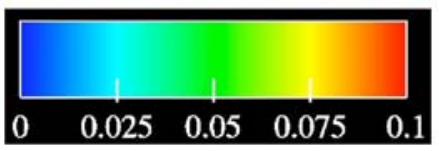

Fig. 5.4. Plastic strain-rate mapping on the interior of the assembly (color scale is in units of $\mu \mathrm{s}^{-1}$ ).

In the actual LSAC assembly, the end caps do not need a very high-pressure buildup to become separated from the liners, as was observed experimentally in Shot 8 . Figure 3.8 shows the extent of the damage suffered by the copper assembly in Shot 8 , where one can clearly see the front-end cap separated from the annular assembly. As can be seen in the photograph, only a small amount of the HE reacted during this shot. Even so, the pressure generated within the assembly was sufficient to remove the end cap in one piece, which indicates weak confinement in the current LSAC assembly design. Thus, one would also expect that the end caps would be ejected prematurely before the entire volume of HE was consumed in the other shots. The end cap failure is from the inability of the threading to sustain the overpressure rather than from a stress concentration around the end cap cylinder junction (Fig. 5.4).

\subsection{Large-Scale Annular Cookoff Assembly Deformation Characteristics of the Inner Liner}

With one-point detonation, perfect symmetry cannot be achieved in practice because the shockwave speeds are finite, although using HEs with high-speed detonation fronts leads to a "more symmetric" collapse than using HEs with lower speeds. However, nearly perfect symmetry could be attained if a large number of detonation points were located around a concentric circle lying in the midplane of the LSAC assembly. The framingcamera images show that during cookoff, there is a single initiation region in all shots 
except Shot 4, where there appeared to be two diametrically opposed initiation regions. Therefore, regardless of the geometrical symmetry of the assembly, initiation did not appear to be confined to any symmetric region within the HE. The data show that at least during the early stages, a circularly symmetric collapse was not observed in any of the shots. Photographic sequences for various shots clearly demonstrate that there is a localized region of the liner that first begins to be accelerated by the expansion of gaseous products. In other words, ignition starts in a relatively small (localized) region of the HE, from which the deflagration front propagates outward. This fact precludes a perfectly symmetric collapse of the inner liner because the metal closest to the initiation region will be subjected to deformation before the metal elements farthest from this region will experience the same level of stress. Therefore, an uneven stress field, which creates an uneven strain-rate field (Fig. 5.4), cannot lead to a symmetric collapse. This will also be true if other regions within the HE ignite at later times. 


\section{Quantification of Symmetry}

Notwithstanding that perfect symmetry is not achievable with one-point detonation, even with pristine PBX-9501, it is not difficult to visualize that different configurations and different kinds of HE (those with lower reaction front velocities, lower peak pressures, different densities, etc.), including those corresponding to cookoff conditions, might lead to "less symmetric" collapses. A natural question that arises when assessing safety is the degree of symmetry that can be achieved by a thermally initiated explosion resulting from cookoff. To establish the degree of symmetry effected by the self-ignition of thermally damaged PBX-9501 (cookoff), we used the one-point detonation simulation with pristine PBX-9501 as our baseline case.

\subsection{A Metric of Symmetry}

There are many different ways of defining a metric to quantify the degree of symmetry. Here, we define a relative geometric measure of symmetry that is based on the time of arrival of the inner liner at selected radial positions, or specific pin-probe locations, as a function of angle. In experiments, pin probes are normally used to determine the arrival times of imploding material. In computations, simulated pins are used to determine when a material interface moves across fixed points in space. Thus, arrival-time versus azimuthal-location curves can be constructed from a set of pins in a given plane. We chose to use a set of 224 pins lying on the midplane, perpendicular to the LSAC axis (see Fig. 6.1), whose polar coordinates $\left(r_{n}, \theta_{m}\right)$ are

$$
\begin{array}{lr}
r_{n} / R_{i}=1-n / 8 & 1 \leq n \leq 7 \\
\theta_{m}=m \pi / 32 & 0 \leq m \leq 32,
\end{array}
$$

where $R_{i}(3.81 \mathrm{~cm})$ is the initial inner radius of the inner liner.

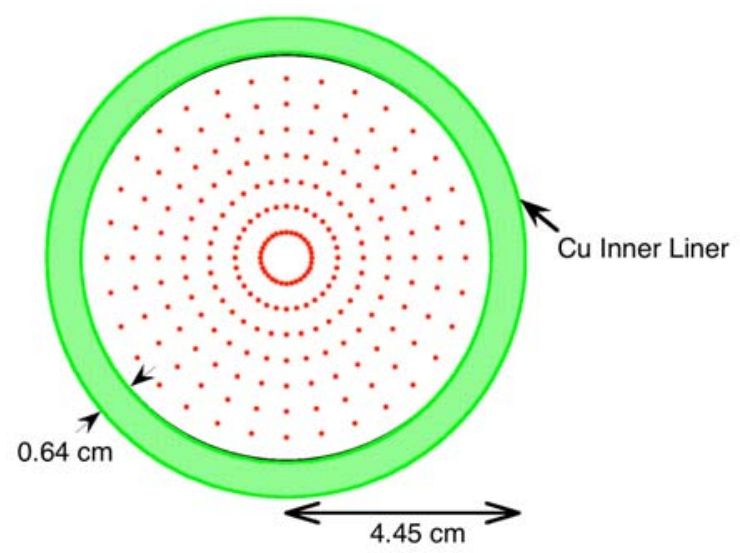

Fig. 6.1. Reference pin locations within the midplane of the assembly.

Our symmetry metric requires that a functional relationship be determined between the time of arrival $t$ at specific pin locations at a fixed radius $r$ and the azimuthal 
location $\theta$; in other words, $t=f(r, \theta)$. Experimentally, it would also be feasible to use 224 pin probes or more to extract detailed arrival-time versus azimuthal-location curves. Since these data were unavailable for all shots, we digitized the contours of the collapsing liner from five unambiguous photographs of Shot 6, which are shown in Fig. 6.2 (and also in Fig. 2.4). These photographs show the spatially integrated view of the collapsing liner along the assembly axis, and therefore, the axial location where the liner is deforming cannot be inferred from these images.

We believe that the photographs taken beyond $70 \mu$ s were obscured by gaseous products (Fig. 6.3) released as a result of end cap failure (Fig. 2.4). However, the experimentalists proposed a different explanation for the irregularly shaped dark areas in the photographs. They have stated that metal, rather than gas products, is what one sees in the field of view. Furthermore, they suggest that the image at $90 \mu \mathrm{s}$ is evidence that the collapse is symmetric. As we discussed in Section 3.2, it is not obvious in Shot 3 whether the obscuring matter was gas, metal, or a combination of the two, without radiographs. However, based on existing photographs (Fig. 6.3) and the distinctive features of the obscuring matter, we think that what is seen at $90 \mu$ s are gaseous products, rather than a metal interface. In addition, the collapse is not symmetric according to the definition of symmetry. Once the collapse begins as asymmetric, it cannot revert to a symmetric one at later times. This is not only physically but also geometrically impossible, as discussed below.

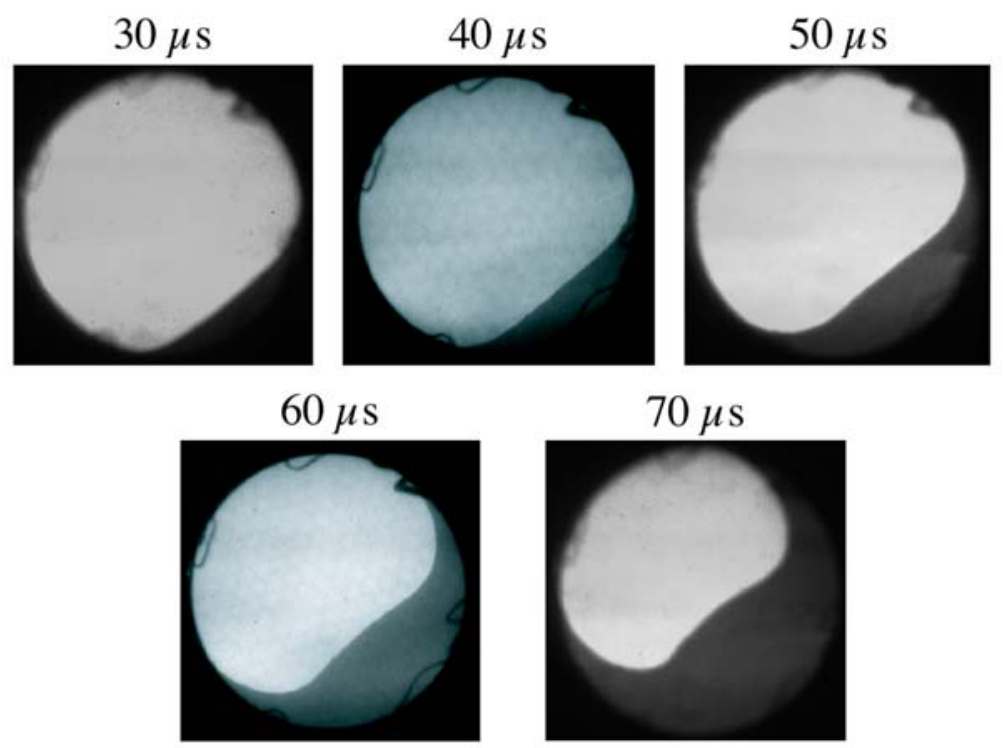

Fig. 6.2. Shot 6 photographs of the inner-liner collapse. 

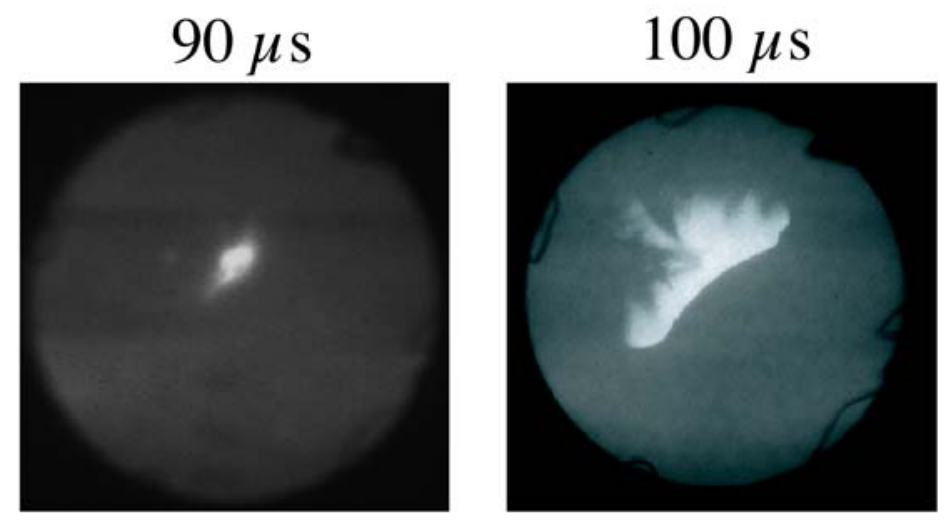

Fig. 6.3. Shot 6 photographs of the inner-liner collapse, obscured by gaseous products.

\subsection{Shot 6 Inner-Liner-Collapse Data Analysis}

Digitization of the collapsing contours of the inner liner in Fig. 6.2 was done by enlarging each frame fourfold and superimposing a polar grid on it (generated by standard plotting software). The polar coordinates of several points on the deformed part of the contours were stored. These data were transformed into Cartesian coordinates to apply smoothing and interpolation routines in order to generate 361 points for each frame. Points in this data set were subsequently transformed into polar coordinates (Fig. 6.4) and used as input to create third-order interpolating functions of the form

$$
r_{k}=g\left(\theta_{l}, t_{k}\right), \quad 1 \leq k \leq 6 \quad 1 \leq l \leq 361 .
$$

To solve numerically for $t$ for a fixed $r$ in the above equation, Newton iteration was used for each $\theta$, resulting in the desired time of arrival to pin locations versus angle curves, which will be referred to as "pin curves." Figure 6.5 shows a comparison between the pin curves obtained from the framing sequence photographed in Shot 6 (red curves) and those from the numerically simulated base case, the one-point detonation with pristine PBX-9501 (blue curves). This comparison is established in terms of relative times of arrival, where time zero is defined as the instant at which the liner first reaches the radial location $r / R_{i}=0.875$. 


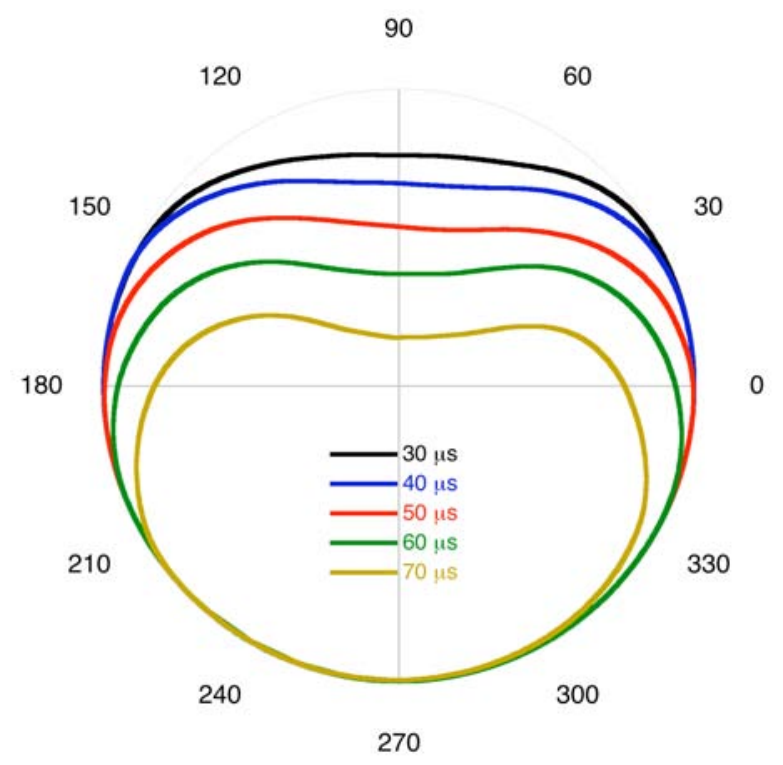

Fig. 6.4. Digitized contours from Shot 6 framing sequence up to $70 \mu \mathrm{s}$.

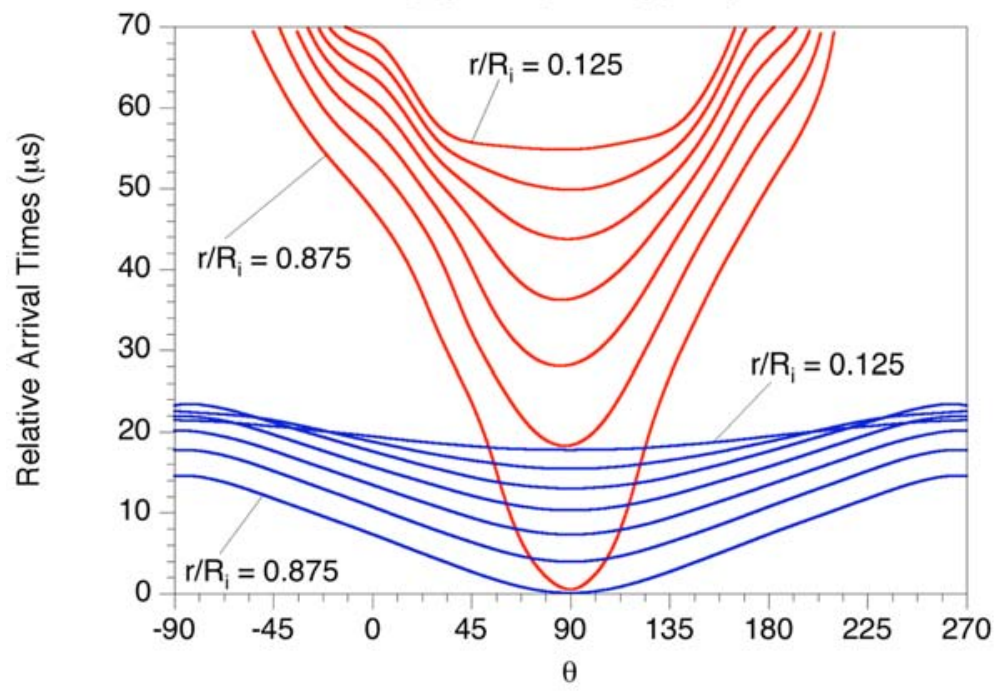

Fig. 6.5. Comparison of the relative time of arrival to pin locations versus angle curves between Shot 6 (red curves) and the one-point detonation numerical simulation with pristine PBX-9501 (blue curves).

Pin curves for a perfectly symmetric collapse would be a series of horizontal straight lines; therefore, any departure from a zero slope in the pin plane is indicative of some asymmetry. Note that in Fig. 6.5, the blue pin curves have, on average, a smaller slope than the pin curves for Shot 6 (red curves). For both families of pin curves, the maximum slope $(d t / d \theta)_{\max }$ remains nearly constant for most radial locations, except for the last one at $r / R_{i}=0.125$. For this curve, 
$(d t / d \theta)_{\max }$ decreases in both the simulation and Shot 6 . Therefore, on the basis of pin curves, the collapsing liner of Shot 6 is less symmetric than that of the one-point detonation base case with pristine HE (i.e., the maximum slope for each red curve is always greater than or equal to the corresponding blue curve). We also digitized some of the images from Shot 3 but the quality of the data was not as good as that for Shot 6 and only the first $25 \mu$ s were unambiguous. Figure 6.6 shows two early pin curves for Shot 3 where the curve irregularity is due to uncertainty in the data. Notice that the collapse of the inner liner in Shot 3 is also asymmetric.

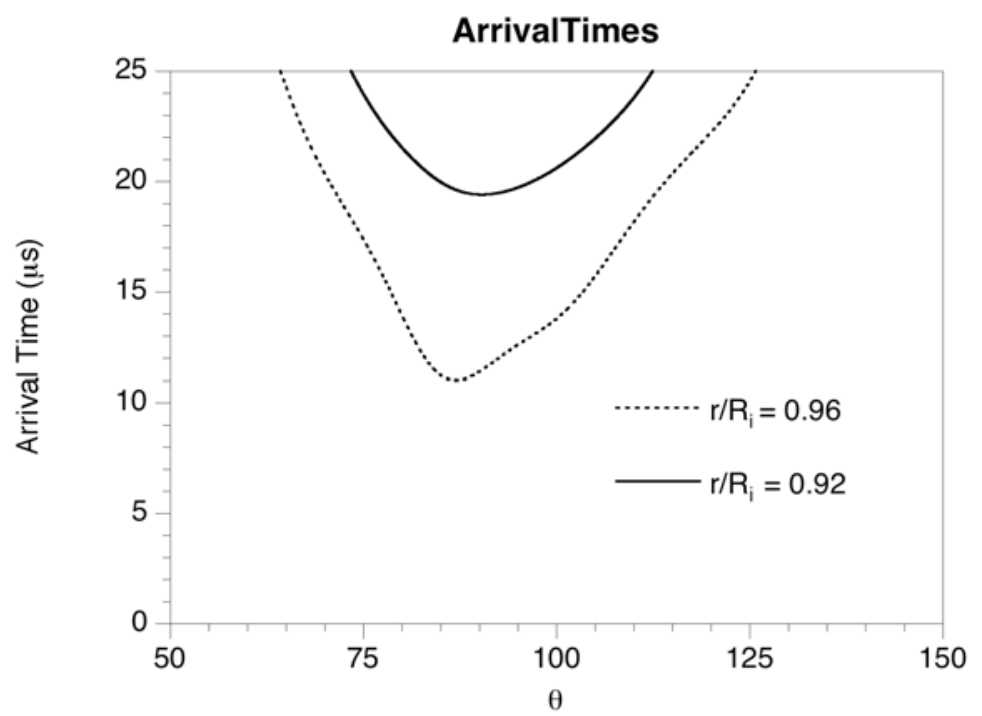

Fig. 6.6. Time of arrival to pin locations versus angle curves for Shot 3. 


\section{Modified Equation of State for Post-Ignition Numerical Simulations}

Because of its simplicity, one of the most commonly used models for simulating energetic materials with hydrocodes is based on the JWL EOS (Fickett and Davis 2000). This is an eight-parameter model that approximates the experimental data from the adiabatic expansion of detonation products, without explicitly including the kinetics of explosion (Ficket and Davis 2000; Explosives Reference Guide 2004). Although the JWL parameters for the detonation of pristine PBX-9501 have been known for some time, the expansion adiabats of the gaseous products produced by thermally damaged PBX-9501 at various heating rates and temperatures have not been characterized to determine whether or not an empirical EOS similar to the JWL could be used to model thermally initiated explosions. If the JWL functional form could describe the adiabatic expansion of deflagrating HE, each adjustable parameter would be at least a function of the thermal history of the cooked HE, and thus, there would be a family of adiabats for different cookoff and confinement conditions.

As a first step in developing a simulation capability for predicting the behavior of cooked HE, we performed a series of 30 simulations with modified versions of the JWL EOS for PBX-9501 and with different HE configurations. In this report, we discuss 20 of these simulations, which we believe best represent our scoping study (see Chapter 8 ). The simulations for Shot 6 were aimed at understanding the time and energy scales and the topology of the inner-liner collapse, as well as quantifying the symmetry through the time of arrival at pin locations versus angle curves. We also performed simulations of Shots 3 and 4 , but since the post-ignition data for these tests were not as detailed as that for Shot 6 , we have not included these results.

From the inner-liner rate of collapse that can be derived from the photographs of Shot 6 (Fig. 6.2), it can be inferred that the reaction front velocity travels, on average, at about $1 \mathrm{~km} / \mathrm{s}$. This low velocity suggests that the reaction propagates as a fast deflagration rather than as a detonation wave. It is important to distinguish, at least qualitatively, the difference between detonation and deflagration before modifying the JWL parameters. Detonations are initiated by shock waves, and therefore the reaction fronts are supersonic. The pressures reached in detonation events vary from tens to hundreds of kilobars. Typical time scales for consumption of the HE are on the order of microseconds $(\sim 10 \mu \mathrm{s})$. By contrast, deflagrations are subsonic events characterized by lower pressures (<10 kbar) than those achieved in detonations of the same energetic material and the $\mathrm{HE}$ is typically consumed within 10 to $1000 \mu \mathrm{s}$ (Explosives Reference Guide 2004). All of the chemical energy stored in a pristine sample of an energetic material can be released by detonation or deflagration. However, 
the rate at which energy is liberated in a detonation is much higher than it is in a deflagration. Thus, modifications to the JWL parameters can be made according to these differing criteria. Under cookoff conditions, before thermal runaway takes place, an HE is thermally decomposed gradually. However, up to this point, the degree of $\mathrm{HE}$ decomposition is relatively small, and most of the chemical energy is still available to perform work on the surroundings. With this in mind, the JWL parameters can be modified according to criteria based on the maximum pressure, available energy, and time scale that are characteristic of the effects produced by the expansion of gaseous products on the surrounding media for a given configuration.

We relied on two approaches to construct consistent sets of JWL parameters that will be described in the next section. In numerical simulations, different parameter combinations will be reflected in the rate of plastic work that the explosion gaseous products exert on the metal liner enclosing an HE. As we will see in Chapter 8, there is a combination of JWL parameters that mimics, with reasonable accuracy (based on the photographic record), the topology of deformation for Shot 6 during the first $70 \mu \mathrm{s}$.

\subsection{Jones-Wilkins-Lee Parameter Modifications}

A brief review of the JWL theory will be necessary to discuss our approaches to modifying the JWL parameters. The EOS used in PAGOSA can be derived from the principal adiabat of detonation products. Following Ficket and Davis (2000), the JWL expansion adiabat in the $p-v$ plane is

$$
p_{a}=A \exp \left(-R_{1} \rho_{\mathrm{o}} v\right)+B \exp \left(-R_{2} \rho_{\mathrm{o}} v\right)+\frac{C}{\left(\rho_{\mathrm{o}} v\right)^{1+\omega}},
$$

where $p_{a}$ is the pressure along the adiabat, $v$ is the specific volume, $\rho_{\mathrm{o}}$ is the density of the unreacted explosive, $A, B$, and $C$ are constants that have units of pressure, and $R_{1}, R_{2}$, and $\omega$ are dimensionless constants.

The constant $\omega$ is the Gruneisen coefficient and is defined by

$$
\omega=\frac{v}{\left(\partial E^{\prime \prime} / \partial p\right)_{v}}
$$

where $E^{\prime \prime}$ is the specific internal energy (energy per unit mass). $E^{\prime \prime}$ can be approximated by a Taylor series expansion in $p$ around the adiabat [Eq. (7.1)]:

$$
E^{\prime \prime}=E^{\prime \prime}\left(p_{a}\right)+\left(\frac{\partial E^{\prime \prime}}{\partial p}\right)_{v}\left(p-p_{a}\right)
$$


Using Eq. (7.2) and resorting to the isentropic relation $\left(d E^{\prime \prime}\left(p_{a}\right) / d v=-p_{a}\right)$, Eq. (7.3) can be rewritten as

$$
E^{\prime \prime}(p, v)=-\int p_{a} d v+\frac{1}{\omega}\left(p-p_{a}\right) v
$$

The functional form of the JWL EOS used in PAGOSA is obtained after carrying out the integration in Eq. (7.4), using Eq. (7.1), and solving for $p$

$$
p=\frac{\omega E^{\prime \prime}}{v}+\left(1-\frac{\omega}{R_{1} \rho_{\mathrm{o}} v}\right) A \exp \left(-R_{1} \rho_{\mathrm{o}} v\right)+\left(1-\frac{\omega}{R_{2} \rho_{\mathrm{o}} v}\right) B \exp \left(-R_{2} \rho_{\mathrm{o}} v\right) .
$$

All of the seven parameters required in Eq. (7.5) $\left(E^{\prime \prime}, \omega, \rho_{\mathrm{o}}, A, B, R_{1}, R_{2}\right)$ can be found for several explosives in the Explosives Reference Guide (2004). The eighth parameter is the detonation velocity $U_{d}$, which appears in the equation for the Rayleigh line

$$
p_{R}=p_{\mathrm{o}}+\left(\frac{1}{\rho_{\mathrm{o}}}-v\right)\left(\rho_{\mathrm{o}} U_{d}\right)^{2}
$$

where $p_{\mathrm{o}}$ is the initial pressure.

At the Chapman-Jouguet (CJ) point, the total specific internal energy in the system $E_{C J}^{\prime \prime}$ is

$$
E_{C J}^{\prime \prime}=E^{\prime \prime}{ }_{\text {chem }}+E_{c}^{\prime \prime},
$$

where $E^{\prime \prime}{ }_{\text {chem }}$ is the specific chemical energy of the HE. $E^{\prime \prime}{ }_{c}$ is the specific compression energy along the Rayleigh line (Cooper 1996; Explosives Reference Guide 2004), which is given by

$$
E_{c}^{\prime \prime}=\frac{1}{2 \rho_{\mathrm{o}}}\left(p_{C J}-p_{\mathrm{o}}\right)\left(1-\rho_{\mathrm{o}} v_{C J}\right)
$$

where $p_{C J}$ is the pressure at the CJ point, which in turn can be written as

$$
p_{C J}=p_{\mathrm{o}}+\rho_{\mathrm{o}} U_{d}^{2}\left(1-\rho_{\mathrm{o}} v_{C J}\right)
$$


A relationship between the constant $C$ in Eq. (7.1) and the other parameters can be found from the identity that results from evaluating the pressure at the CJ point using Eqs. (7.1) and (7.5) [i.e., $\left.p_{a}\left(v=v_{C J}\right)=p\left(v=v_{C J}, E^{\prime \prime}=E^{\prime \prime}{ }_{C J}\right)\right]$.

$C=\omega V_{C J}^{\omega}\left\{E_{\text {chem }}-\frac{A}{R_{1}} \exp \left(-R_{1} V_{C J}\right)-\frac{B}{R_{2}} \exp \left(-R_{2} V_{C J}\right)+\frac{1}{2 \rho_{\mathrm{o}}}\left(p_{C J}-p_{\mathrm{o}}\right)\left(1-V_{C J}\right)\right\}$,

where $E_{\text {chem }}=\rho_{\mathrm{o}} E^{\prime \prime}{ }_{\text {chem }}$ (energy per unit volume, which has units of pressure), and $V_{C J}=\rho_{\mathrm{o}} v_{C J}$ (relative specific volume, which is dimensionless).

To generate a JWL EOS that approximates the deflagration behavior of PBX-9501, we start with the set of eight experimentally determined parameters that characterize the detonation of pristine PBX-9501. Below, we discuss two approaches that can be used to modify these "original" parameters.

\subsubsection{First Approach}

The JWL parameters can be modified by enforcing a preselected reaction-front velocity and density, while at the same time preserving the monotonicity of the original adiabat. This modification will necessarily decrease the available chemical energy, which needs to be calculated. One can ensure that the monotonicity of the adiabat will be the same by retaining the same power law decay in the last term of Eq. (7.1) and keeping the original exponents $R_{1}$ and $R_{2}$. To reduce to a minimum the number of conditions imposed on the modified JWL, we scale the original adiabat, Eq. (7.1), by a factor $\alpha$. At the CJ point, the adiabat and the Rayleigh line intersect, and their slopes are equal; thus, to ensure thermodynamic consistency with simple detonation theory (Ficket and Davis 2000), the following conditions are satisfied:

$$
\begin{gathered}
\left.\alpha p_{a}\right|_{C J}=\left.p_{R}\right|_{C J} \\
\left.\frac{\partial\left(\alpha p_{a}\right)}{\partial v}\right|_{C J}=\left.\frac{\partial p_{R}}{\partial v}\right|_{C J} .
\end{gathered}
$$

We denote the adjustable parameters for the new adiabat with an asterisk. From the system of Eqs. (7.11) and (7.12), we solve for $\alpha$ as follows:

$$
\alpha=\frac{(1+\omega) p_{o}+\rho_{o} U_{d}^{2}\left[1+\omega-(2+\omega) \rho_{o} v_{C J}\right]}{\left(1+\omega-\rho_{o} R_{1} v_{C J}\right) A e^{-\rho_{o} R_{1} v_{C J}}+\left(1+\omega-\rho_{o} R_{2} v_{C J}\right) B e^{-\rho_{o} R_{2} v_{C J}}} .
$$


Thus, for the new adiabat, $A^{*}=\alpha A$ and $B^{*}=\alpha B$. Solving for $C^{*}$ after neglecting $p_{o}$, we obtain

$$
C^{*}=\left(\rho_{o} v_{C J}\right)^{2+\omega} \frac{\left[\left(1-R_{1}-\rho_{o} R_{1} v_{C J}\right) \rho_{o} U_{d}^{2}\right] A^{*} e^{-\rho_{o} R_{1} v_{C J}}+\left[\left(1-R_{2}-\rho_{o} R_{2} v_{C J}\right) \rho_{o} U_{d}^{2}\right] B^{*} e^{-\rho_{o} R_{2} v_{C J}}}{\left[1+\omega-(2+\omega) \rho_{o} v_{C J}\right] \rho_{o} U_{d}^{2}} .
$$

We note that the value of the product $\rho_{o} v_{C J}$ is on the order of 0.75 for all explosives (Explosives Reference Guide 2004). As an application of the preceding equations, consider the following example in which we fix the reaction-front velocity and the density of the unreacted explosive to $6 \mathrm{~km} / \mathrm{s}$ and $1.8 \mathrm{~g} / \mathrm{cm}^{3}$, respectively. Table 7.1 shows the adiabat parameters for pristine $\mathrm{PBX}-9501$ and the second column a modified (degraded) HE. Since we reduced the detonation velocity without imposing any constraint on the maximum amount of work that the expansion of gaseous products can perform, the specific internal energy will change from that of the original HE. This new energy can be calculated by solving for $E_{\text {chem }}$, using Eq. (7.10). Figure 7.1 shows the expansion adiabats and their respective Rayleigh lines for the detonation products of PBX-9501 as well as those corresponding to the degraded HE considered in the example above.

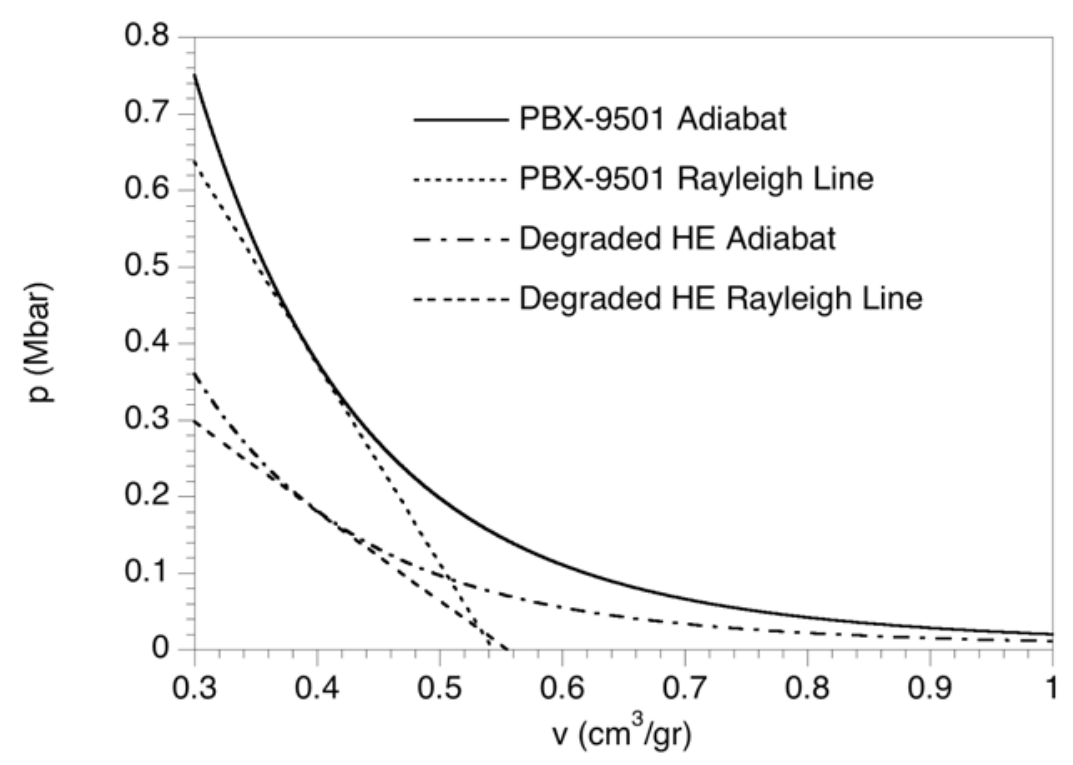

Fig. 7.1. Expansion adiabats and their respective Rayleigh lines for the detonation products of PBX-9501 and degraded HE (see Table 7.1).

\subsubsection{Second Approach}

Our second approach to constructing consistent JWL parameters is based on enforcing the fact that the specific chemical energy is the same for cooked HE as it is for 
pristine PBX-9501, while at the same time, the propagation velocity of the reaction front is decreased. We relied mostly on the second approach, in which the monotonicity of the modified adiabat might differ from that for pristine PBX-9501. By definition, the chemical energy of the HE is equal to the maximum amount of work per unit mass that can be drawn from the expansion adiabat of gaseous products. Thus,

$$
E^{\prime \prime}{ }_{\text {chem }}=\int_{v_{0}}^{\infty} p_{a} d v-E_{c}^{\prime \prime}
$$

where $E^{\prime \prime}{ }_{c}$ is the energy required to reach the CJ point and is given by Eq. (7.8).

From Eq. (7.15), we see that the chemical energy per unit volume, in terms of the relative specific volume, becomes

$$
E_{c h e m}=\frac{A}{R_{1}} \exp \left(-R_{1} V_{C J}\right)+\frac{B}{R_{2}} \exp \left(-R_{2} V_{C J}\right)+\frac{C}{\omega V_{C J}^{\omega}}-\frac{1}{2 \rho_{\mathrm{o}}}\left(p_{C J}-p_{\mathrm{o}}\right)\left(1-V_{C J}\right)
$$

Notice that the above equation could also have been obtained by solving for $E_{\text {chem }}$ from Eq. (7.10). To be consistent with the JWL theory, the pressure reached after the HE is compressed from its initial state $v_{0}$ to $v_{C J}$ must be the same as the pressure evaluated with the expansion adiabat at the $\mathrm{CJ}$ point

$$
\begin{gathered}
\left.p_{a}\right|_{C J}=\left.p_{R}\right|_{C J} \\
\left.\frac{\partial p_{a}}{\partial v}\right|_{C J}=\left.\frac{\partial p_{R}}{\partial v}\right|_{C J} .
\end{gathered}
$$

In other words, at the CJ point, the Rayleigh line intersects and is tangent to the expansion adiabat of the detonation products. For a known detonation velocity and initial density, the conditions at the CJ point can be calculated as follows from experimental correlations (Cooper 1996).

$$
\begin{gathered}
v_{C J}=\frac{0.7125}{\rho_{0}^{0.96}} \\
p_{C J}=\rho_{0} U_{d}^{2}\left(1-0.7125 \rho_{0}^{0.04}\right),
\end{gathered}
$$


where $\rho_{0}$ is in $\mathrm{g} / \mathrm{cm}^{3}$.

Substituting Eqs. (7.19) and (7.20) into Eqs. (7.16) to (7.18) results in a system of three linear equations that are linear in $A, B$, and $C$. Although the solution of this system of equations is straightforward, the resulting expressions are lengthy, so they are not included here.

If the corresponding values of actual PBX-9501 are used for $E^{\prime \prime}, \omega, \rho_{0}, R_{1}, R_{2}$, and a propagation velocity of $1 \mathrm{~km} / \mathrm{s}$ is assumed, the resulting parameters for the new expansion adiabat are shown in Table 7.1. In this instance, the maximum pressure that can be reached by the explosion products is $11 \mathrm{kbar}$. If necessary, the maximum pressure can be set to a predetermined level by adjusting the values of the exponents $R_{1}$ and $R_{2}$, as shown in the last column of Table 7.1, where the maximum pressure was lowered to 8 kbar.

Table 7.1. JWL Parameters for PBX-9501 and an Artificially Degraded HE

\begin{tabular}{|l|l|c|c|c|}
\hline \multicolumn{1}{|c|}{ Parameters } & PBX-9501* & $\begin{array}{c}E_{\text {chem }}<E_{P B X-9501} \\
U_{d}=6 \mathrm{~km} / \mathrm{s}\end{array}$ & $\begin{array}{c}E_{\text {chem }}=E_{P B X-9501} \\
U_{d}=1 \mathrm{~km} / \mathrm{s}\end{array}$ & $\begin{array}{c}E_{\text {chem }}=E_{P B X-9501} \\
U_{d}=1 \mathrm{~km} / \mathrm{s}\end{array}$ \\
\hline$A(\mathrm{Mb})$ & 6.639895 & 3.034535 & 0.150934 & -0.28598 \\
\hline$B(\mathrm{Mb})$ & 0.3766498 & 0.172135 & -0.297886 & -0.17180 \\
\hline$R_{1}$ & 4.40325 & 4.40325 & 4.40325 & 4.6 \\
\hline$R_{2}$ & 1.9 & 1.9 & 1.9 & 1.3 \\
\hline$C(\mathrm{Mb})$ & 0.014926 & 0.006509 & 0.047528 & 0.052765 \\
\hline$\omega$ & 0.38 & 0.38 & 0.38 & 0.38 \\
\hline$\rho_{0}\left(\mathrm{~g} / \mathrm{cm}^{3}\right)$ & 1.84 & 1.80 & 1.84 & 1.84 \\
\hline$U_{d}(\mathrm{~km} / \mathrm{s})$ & 8.79 & 6 & 1 & 1 \\
\hline$E^{\prime \prime}{ }_{\text {chem }}\left(\mathrm{Mb} \mathrm{cm}{ }^{3} / \mathrm{g}\right) \dagger$ & 0.05571 & 0.0327 & 0.05571 & 0.05571 \\
\hline$E_{\text {chem }}(\mathrm{Mb})$ & 0.1025 & 0.0589 & 0.1025 & 0.1025 \\
\hline$P_{\max }(\mathrm{Mb})$ & $0.39^{* *}$ & 0.18 & 0.011 & 0.008 \\
\hline
\end{tabular}

${ }^{*}$ Explosives Reference Guide 2004

$\dagger$ The specified HE chemical energy in PAGOSA is given in units of energy per unit mass $\left(E_{c h e m}^{\prime \prime}\right)$.

${ }^{* *} \mathrm{CJ}$ pressure

Figure 7.2 shows a comparison between the expansion adiabat of the detonation products for PBX-9501 and a deflagration approximant based on the parameters listed in the last column of Table 7.1. Using the second approach described above, we generated several EOSs to approximate deflagration, with front velocities of $1,1.4,2$, and $4 \mathrm{~km} / \mathrm{s}$, and implemented them in PAGOSA. As we discuss in Chapter 8, the JWL that best described the experimental data was modified according to the second approach, with a reaction-front velocity of $1 \mathrm{~km} / \mathrm{s}$. We emphasize that we adopted the JWL EOS for modeling the post-ignition behavior of a thermally initiated explosion for the sake of convenience and ease of use in the present scoping study, since the model was already implemented in PAGOSA. So far, there is no deflagration model for thermally initiated explosions with the same generality that the JWL theory has for the detonation of explosives. 
Our modified JWL is used to approximate a hypothetical deflagration process, and as we will see in Chapter 8, the resulting simulation reproduces the main features observed in the collapse of the inner liner in Shot 6. A more detailed model will have to await further characterization of cooked HE. Meanwhile, we have considered applying a bulkburn reaction model coupled to the Forest fire model that allows for initially subsonic reaction rates of propagation as a function of pressure to simulate post-ignition in cookoff experiments.

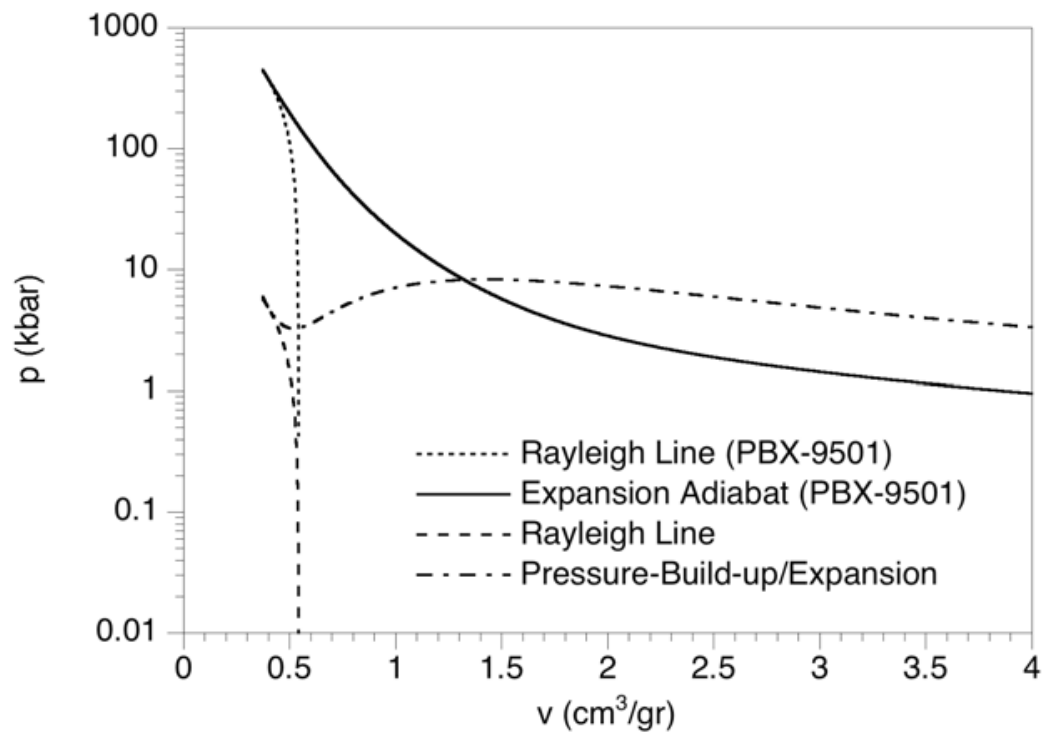

Fig. 7.2. Expansion adiabat and Rayleigh line for detonation of PBX-9501 with the expansion adiabat for deflagration of PBX-9501 (see Table 7.1).

\subsection{Is There a Strong Correlation between Deflagration Speed and Temperature?}

As shown by the thermocouple records for all the shots, the temperature distribution is neither radially nor azimuthally uniform within the HE. With only 18 thermocouples, it is not possible to construct temperature contours in the HE region with a reasonable degree of accuracy. However, pre-ignition simulations can give us an idea of the temperature distribution before thermal runaway (Zerkle and Luck 2003; Aviles-Ramos 2004). It can be argued that nonuniformity in the level of thermal decomposition and in certain other characteristics of the degraded explosive might lead to deflagration zones with different propagation speeds.

To test that hypothesis, we simulated explosions of the LSAC assemblies that had cylindrical layers of $\mathrm{HE}$ whose cross sections resembled the isotherms calculated by other authors (Zerkle and Luck 2003; Aviles-Ramos 2004). These temperature contours are only approximations to what actually might have occurred in the LSAC experiments because there is not enough data to create a detailed temperature map with the degree of accuracy that can be achieved with numerical simulations. Therefore, these approximations could be very different from any that occurred in Shot 6 or any other shots. We used only these approximated temperature contours to qualitatively ascertain whether or not there is a correlation between the temperature distribution and any 
deflagration speed nonuniformity on the collapse of the inner liner. Figure 7.3 is a diagram showing a cross section of the HE with an arrangement consisting of four explosives, each with a different detonation velocity, bounded by three approximated isotherms.

In our computational models, isotherm contours were approximated by "stretching" ellipses around circles, according to the following functional form:

$$
y=\left(a_{1}-x^{2}\right)^{1 / 4}\left[a_{2} \pm a_{3}\left(1-\frac{x^{2}}{a_{4}}\right)^{1 / 2}\right]^{1 / 2}
$$

where the constants $a_{i}$ depend on the location and the radial and angular extents of the isotherm. An isotherm can be extended beyond the first quadrant after transforming its coordinates, obtained from the equation above, into polar form (where they are easily stretched further).

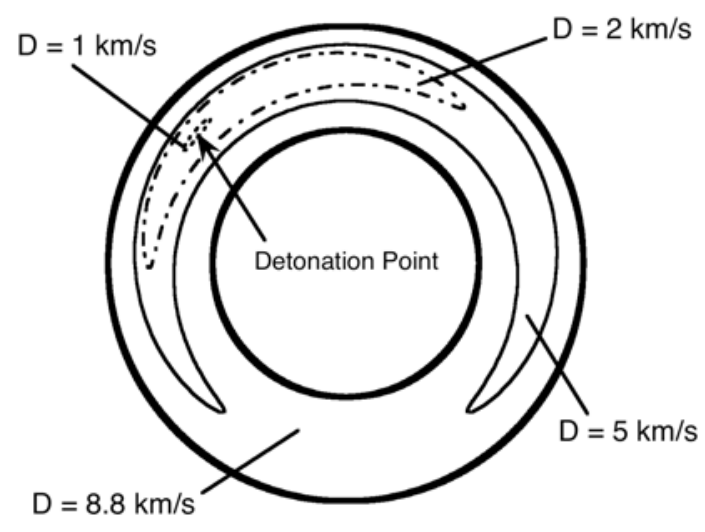

Fig. 7.3. Cross section of a four-layered LSAC assembly HE model.

In later simulations, the configurations we simulated were differentiated by the number and location of initiation points, the geometrical arrangement of $\mathrm{HE}$, and the modified JWL parameters. In all cases, the various regions of HE extend uniformly along the axial direction, and the initiation points are always located on the midplane of the assembly. Thus, a cross section is sufficient to depict the geometrical arrangement of the $\mathrm{HE}$ and detonation points.

Before we started our scoping calculations, we performed several simulations using the configuration shown in Fig. 7.3 to identify the effect of abrupt detonation velocity changes and to determine whether the propagation of the reaction front correlates in any way with the isotherms. Our first choice for the velocities of these preliminary simulations was somewhat arbitrary. Figure 7.4 shows the collapse of the inner liner 
viewed from the top of the LSAC assembly through its axis. Since the inner liner deforms differently in this figure than it does in the framing camera images of Shot 6 (Figs. 6.2 and 6.4), the distribution of propagation velocities in Fig. 7.3 is unlikely to be an accurate model for any of the shots tested. The unambiguous photographs of Shots 3, 4, and 6, where the gas is not in the field of view, do not show the kind of asymmetric collapse displayed in Fig. 7.4. Because we are seeking HE configurations that can reproduce the topology of the inner-liner collapse and the arrival times to specified pin locations, the above configuration was ruled out-it cannot reproduce the contour sequence of Fig. 6.4. In Chapter 8, we will discuss the simulation results for a series of different configurations aimed at approximating the behavior of Shot 6 .
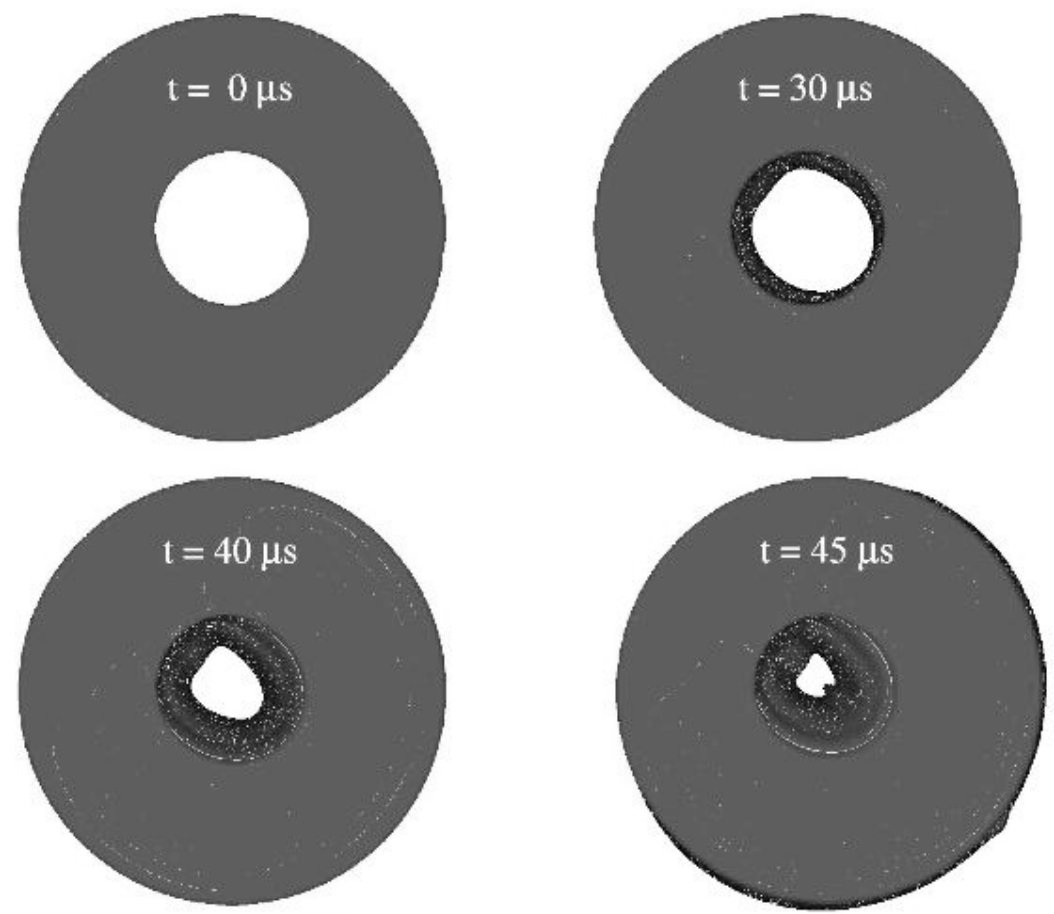

Fig. 7.4. Inner-liner collapse sequence for the configuration depicted in Fig. 7.3. 


\section{Inner-Liner Collapse: Numerical Simulation Results}

In this chapter, we present the salient features of simulations that were performed on the LSAC geometry for Shot 6 to quantify the symmetry of the inner-liner collapse. These cases represent a wide range of configurations for the initiation regions (all of which are confined to the midplane of the assembly) and for degraded HE arrangements whose distribution is independent of the axial coordinate. We have selected the most representative cases to illustrate how varying the reaction front velocity, available chemical energy, and initiation region affects the collapse of the inner liner. Our primary focus is on quantifying the symmetry by computing the functional dependence between the relative time of arrival to pin locations as a function of azimuthal location, referred to as pin curves (see Chapter 6). In each figure, the computed pin curves are compared with those experimentally obtained for Shot 6 . To help pair each case with its corresponding family of pin curves, the cross section of the HE configuration is displayed in each figure. Additionally, we discuss the azimuthal distribution of pressure and compression in the inner liner for two cases: a configuration with an approximate deflagration EOS (see Chapter 7) that mimics the collapse of Shot 6 and the one-point detonation base-case configuration for pristine PBX-9501. The azimuthal nonuniformity of both the pin curves and the pressure distribution around the inner liner conclusively establish, in our view, that the liner collapse in Shot 6 was not symmetric.

\subsection{Pin-Curve Analysis}

Figure 8.1 shows the pin curves for a configuration where initiation took place at a single point located near the outer liner. The entire HE is described by a modified JWL with a reaction front velocity of $1 \mathrm{~km} / \mathrm{s}$ and $2 \%$ of the available chemical energy in

pristine PBX-9501 $\left(E_{P B X-9501}\right)$. Because of the low level of energy that can be released with this set of JWL parameters, the rate of collapse was substantially lower in the simulation than it was in the experiment for Shot 6 . As a result, the pin curves are too far apart for there to be agreement between the two, as shown in Fig. 8.1. However, there is agreement between the computations and experiment on the curvature of the pin curves at $r / R_{i}=0.875$, in an $80^{\circ}$ sector from $50^{\circ}$ to $130^{\circ}$. This means that within a small angular region, the topology of the collapsing liner is initially similar in both. 


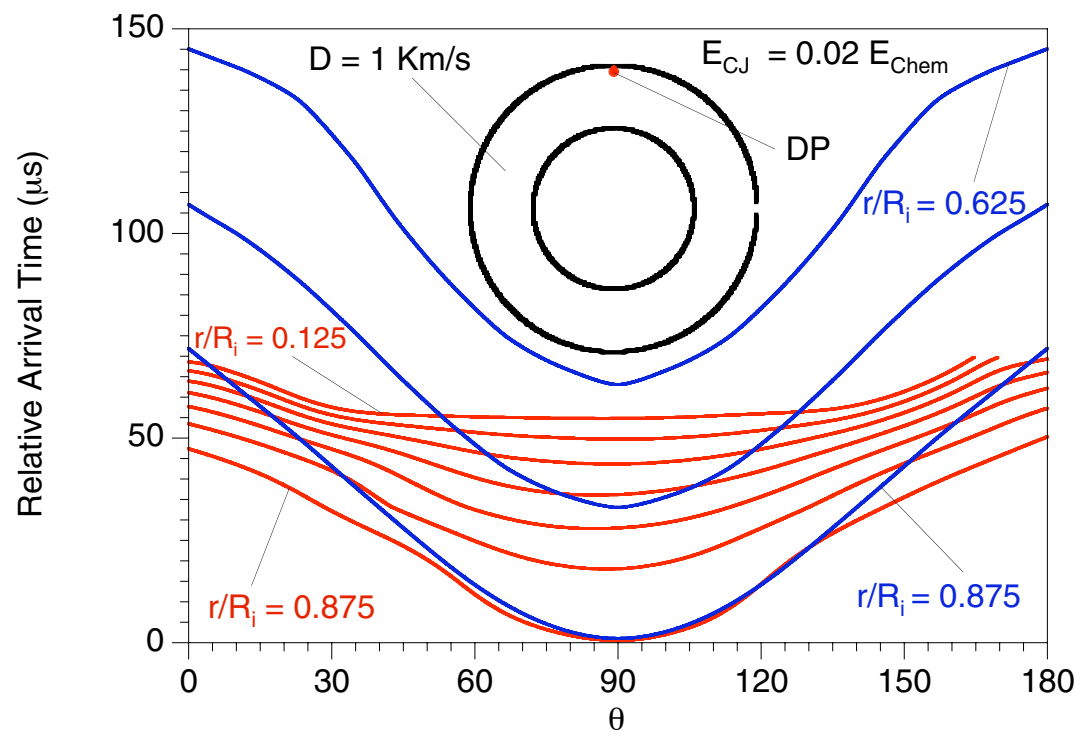

Fig. 8.1. Pin curves for a simulated configuration (blue) initiated at a point near the outer liner with $\mathrm{D}=1 \mathrm{~km} / \mathrm{s}$ and $2 \%$ of $E_{P B X-9501}$. Shot 6 pin curves are shown in red.

For the configuration represented in Fig. 8.2, the available energy has been increased to $50 \%$ of $E_{P B X-9501}$, while the reaction front velocity has remained at $1 \mathrm{~km} / \mathrm{s}$ and the location of the initiation point has not changed (note that the change in scale makes the data appear flatter compared with Fig. 8.1). This increase in energy brought the pin curves slightly closer to the experimental curves as shown in Fig. 8.2. However, the discrepancy in timing and slope is considerable, suggesting that the actual energy released in Shot 6 was probably higher. When the available energy is increased to $100 \%$ of $E_{P B X-9501}$, the timing of the simulated pin curves is comparable to the timing in Shot 6, as shown in Fig. 8.3. In this case, where the initiation point was located near the inner liner and the reaction front velocity was $1 \mathrm{~km} / \mathrm{s}$, the slopes of the pin curves for Shot 6 are steeper during the early times and approach the computed slopes at later times. 


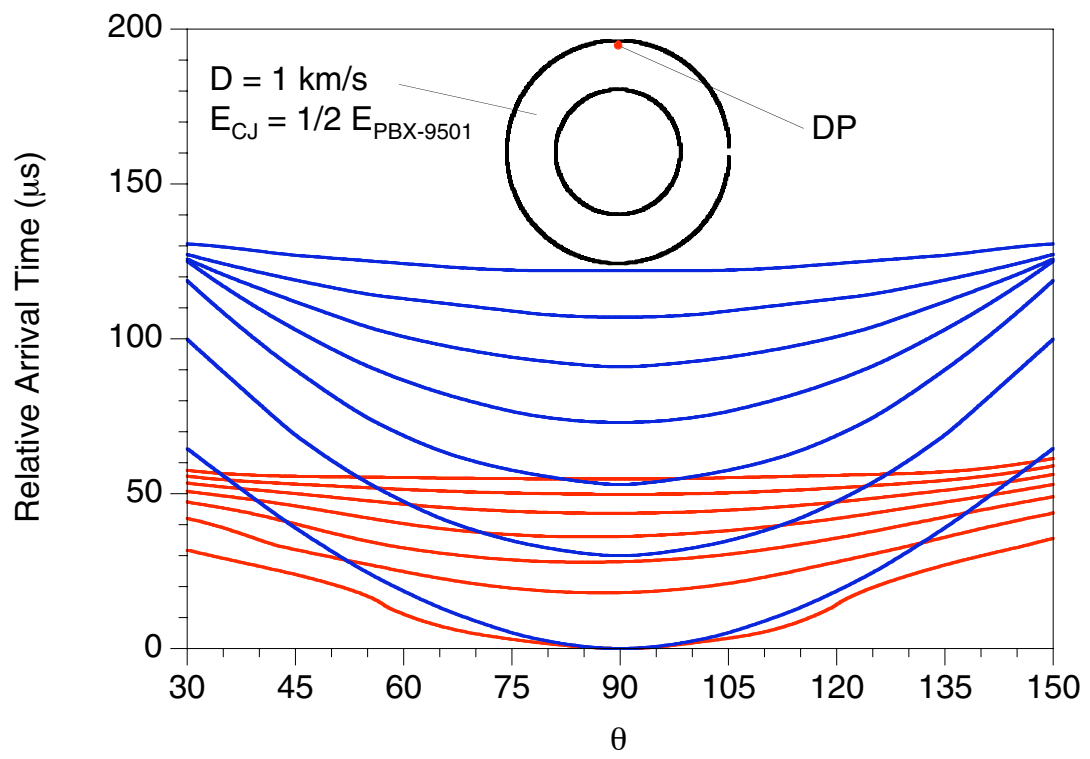

Fig. 8.2. Pin curves for a simulated configuration (blue) with $D=1 \mathrm{~km} / \mathrm{s}$ and $50 \%$ of $E_{P B X-9501}$. The initiation point is located near the outer liner. Shot 6 pin curves are shown in red.

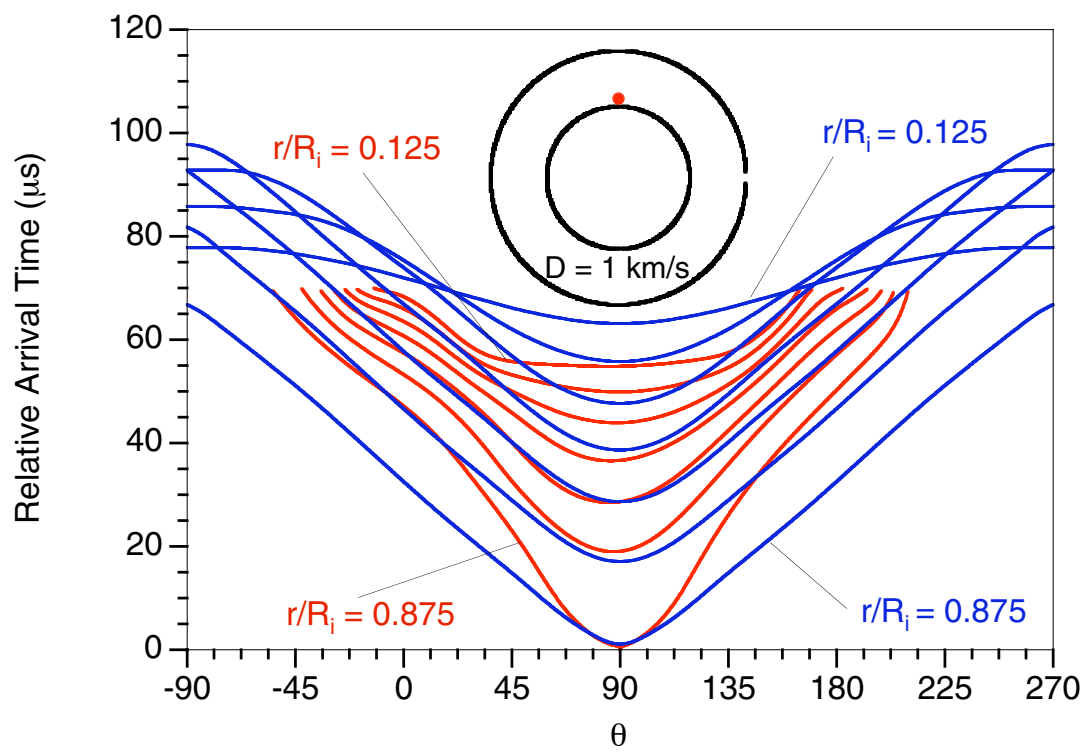

Fig. 8.3. Pin curves for a simulated configuration (blue) with $D=1 \mathrm{~km} / \mathrm{s}$ and $100 \%$ of $E_{P B X-9501}$. The initiation point is located near the inner liner. Shot 6 pin curves are shown in red.

Figure 8.4 shows the pin curves for a configuration in which initiation occurred at a single point midway between the inner and outer liners, about $1.5 \mathrm{~cm}$ from the outer liner. The entire HE is described by a modified JWL with a reaction front velocity of $1 \mathrm{~km} / \mathrm{s}$ and $100 \%$ of $E_{P B X-9501}$. This case is very similar to the one shown in Fig. 8.3 (which 
deviates considerably from the data) except for a slightly faster motion of the inner liner that makes the pin curves shift downward.

The pin curves in Fig. 8.5 correspond to a configuration that is identical to the one shown in Fig. 8.1 except that now $100 \%$ of $E_{P B X-9501}$ is available. Comparison between the first pin curve $\left(r / R_{i}=0.875\right)$ of Shot 6 and that of the simulation show good agreement within the sector that extends from $45^{\circ}$ to $155^{\circ}$. In addition, there is reasonable timing agreement between the pin curves at $r / R_{i}=0.875$ and $r / R_{i}=0.75$. However, beyond the pin curve at $r / R_{i}=0.625$, the remaining curves increasingly depart in time and slope. The cases depicted in Figs. 8.3 through 8.5 illustrate the sensitivity of the pin curves to the radial location of the initiation region. This departure may be explained by the size difference in the radius of curvature of the pressure front before it collides with the inner liner, which strongly depends on the location of the initiation point. When the initiation point is near the outer liner, the radius of curvature of the pressure front has more time to grow radially; thus, by the time it collides against the inner liner, it has developed a flatter front than it would have if the initiation point were closer to the liner. Figure 8.6 shows the pin curves for a configuration in which initiation occurs at a single point near the outer liner and the entire HE is described by a modified JWL with a reaction front velocity of $4.4 \mathrm{~km} / \mathrm{s}$ and $46 \%$ of $E_{P B X-9501}$. The combined effect of reducing the detonation velocity and the available chemical energy of the pristine HE by a factor of two accelerated the metal much faster than what was observed in Shot 6. In this case, the timing of the pin curves is approximately twice that calculated for the pristine configuration shown in Fig. 6.5.

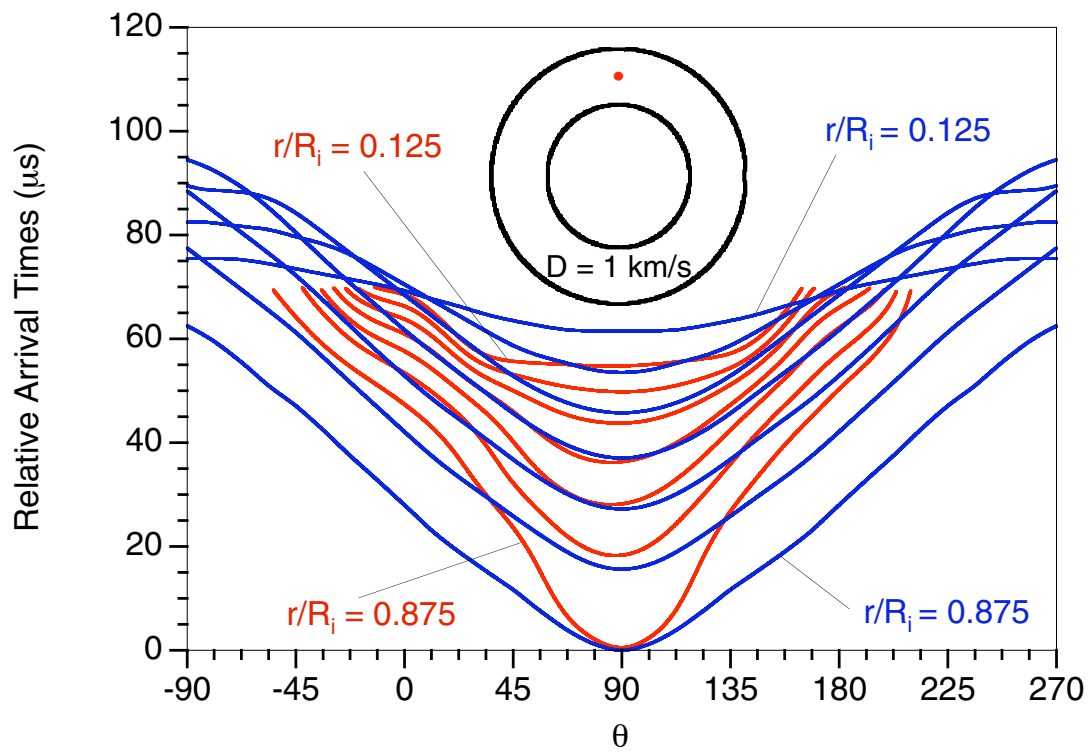

Fig. 8.4. Pin curves for a simulated configuration (blue) with $D=1 \mathrm{~km} / \mathrm{s}$ and $100 \%$ of $E_{P B X-9501}$. The initiation point is located at the midpoint. Shot 6 pin curves are shown in red. 


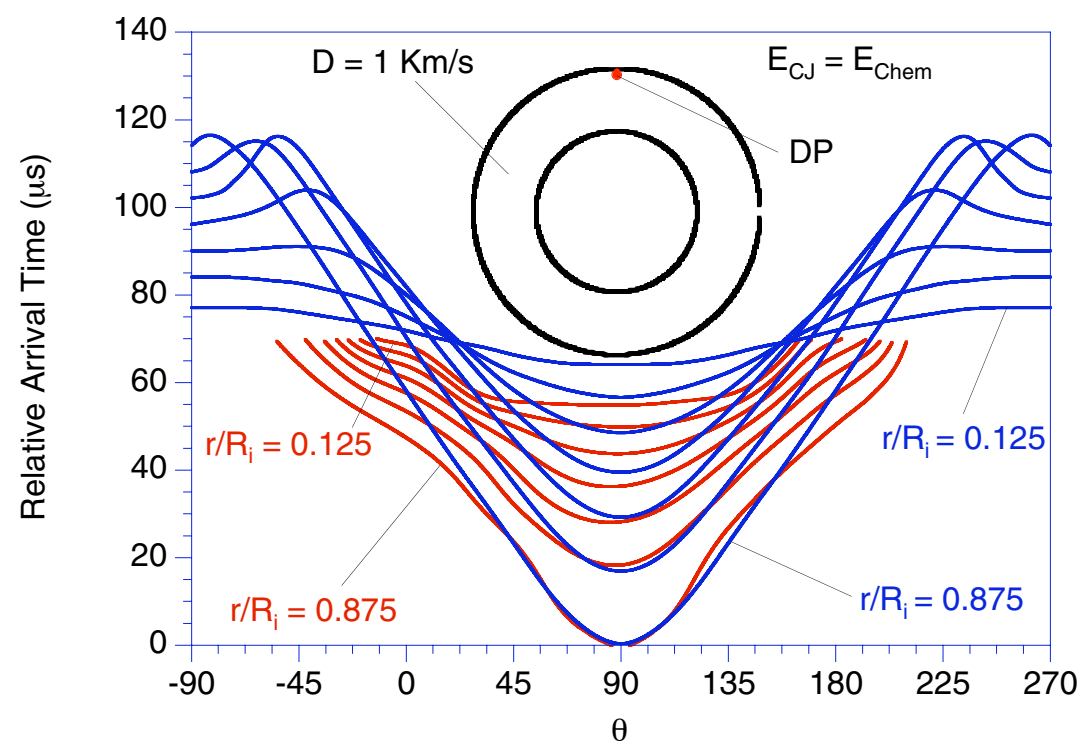

Fig. 8.5. Pin curves for a simulated configuration (blue) with $D=1 \mathrm{~km} / \mathrm{s}$ and $100 \%$ of $E_{P B X-9501}$. The initiation point is located at the outer liner. Shot 6 pin curves are shown in red.

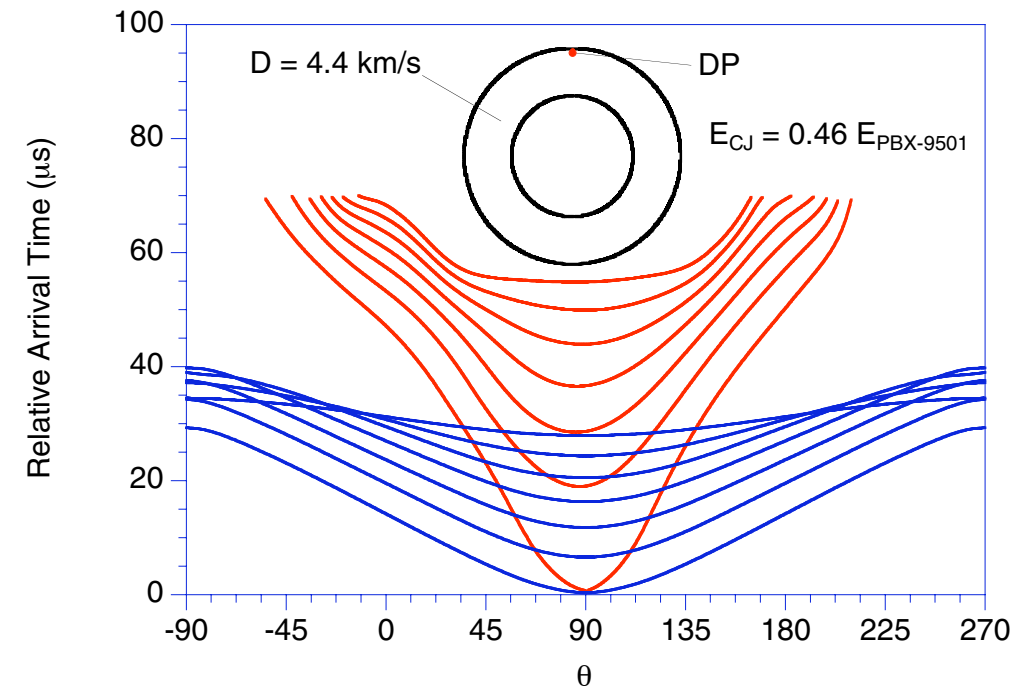

Fig. 8.6. Pin curves for a simulated configuration (blue) with $D=4.4 \mathrm{~km} / \mathrm{s}$ and $46 \%$ of $E_{P B X-9501}$. The initiation point is located at the outer liner. Shot 6 pin curves are shown in red.

Figure 8.7 shows the pin curves corresponding to a configuration in which initiation is at a single point near the outer liner and the entire HE is described by a modified JWL with a reaction front velocity of $1.4 \mathrm{~km} / \mathrm{s}$ and $100 \%$ of $E_{P B X-9501}$. The increase in propagation velocity from $1 \mathrm{~km} / \mathrm{s}$ to $1.4 \mathrm{~km} / \mathrm{s}$ did not improve the reasonably good agreement found for the configuration shown in Fig. 8.5. However, the late-time behavior is closer to that of Shot 6 . The pin curves in Fig. 8.8 correspond to a configuration similar 
to that in Fig. 8.7, except that the propagation velocity was increased to $2 \mathrm{~km} / \mathrm{s}$. In this case, the overall agreement with the data from Shot 6 is poor because of the downward shifting of the computed pin curves.

To calculate the pin curves shown in Fig. 8.9, we first computed the isobars generated by a one-point initiation case with a reaction front velocity of $1 \mathrm{~km} / \mathrm{s}$ and $100 \%$ of $E_{P B X-9501}$. We chose the isochor whose leading edge was located $1.16 \mathrm{~cm}$ from the inner liner and placed 30 initiation points along its locus. In this configuration, the timing of the minima for the first five pin curves is closer to Shot 6 than the case configuration shown in Fig. 8.5.

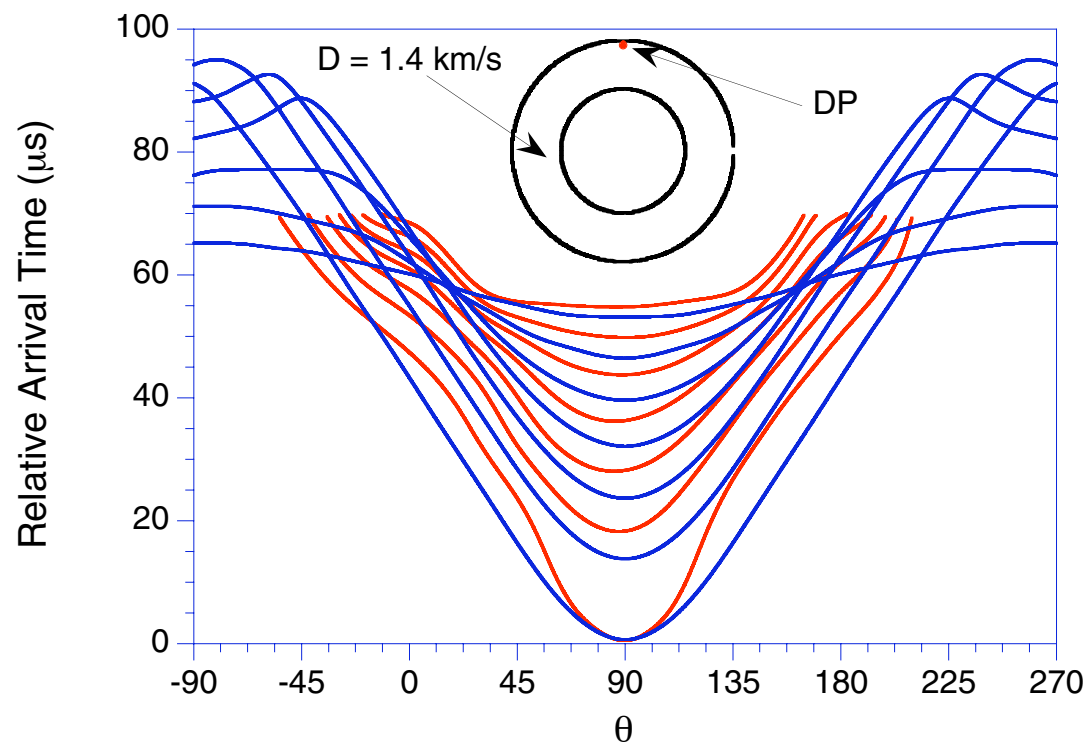

Fig. 8.7. Pin curves for a simulated configuration (blue) initiated at a point near the outer liner with $\mathrm{D}=1.4 \mathrm{~km} / \mathrm{s}$ and $100 \%$ of $E_{P B X-9501}$. Shot 6 pin curves are shown in red. 


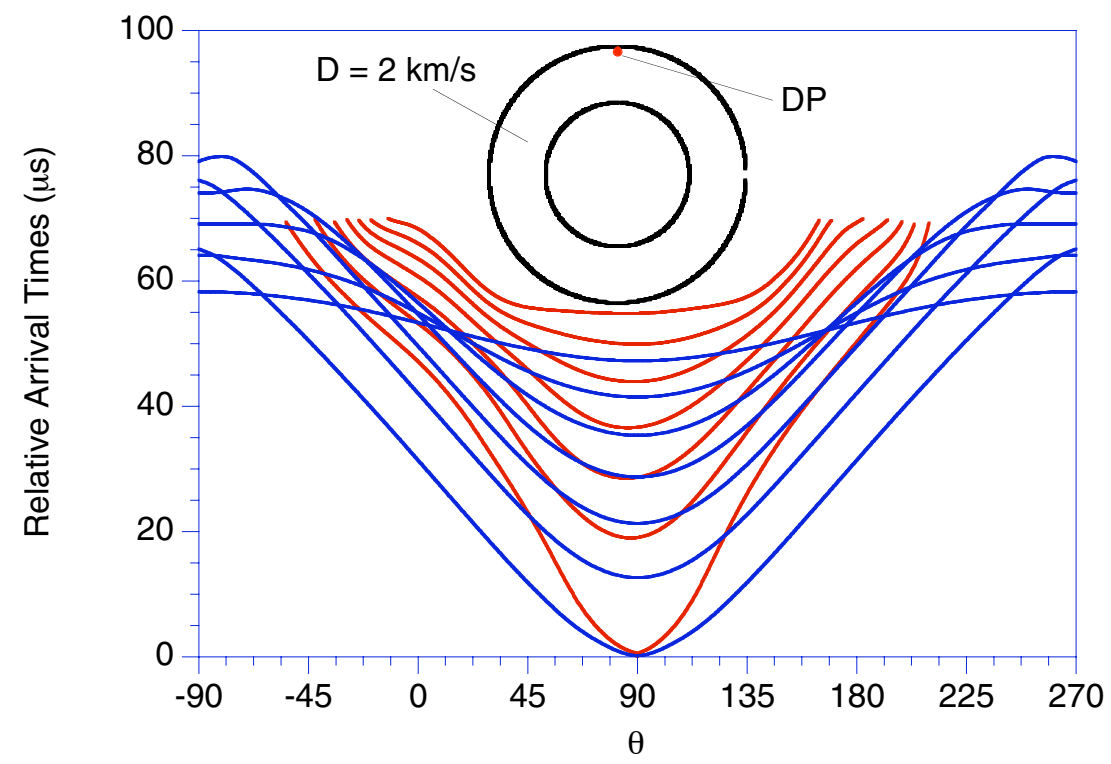

Fig. 8.8. Pin curves for a simulated configuration (blue) initiated at a point near the outer liner with $\mathrm{D}=2 \mathrm{~km} / \mathrm{s}$ and $100 \%$ of $E_{P B X-9501}$. Shot 6 pin curves are shown in red.

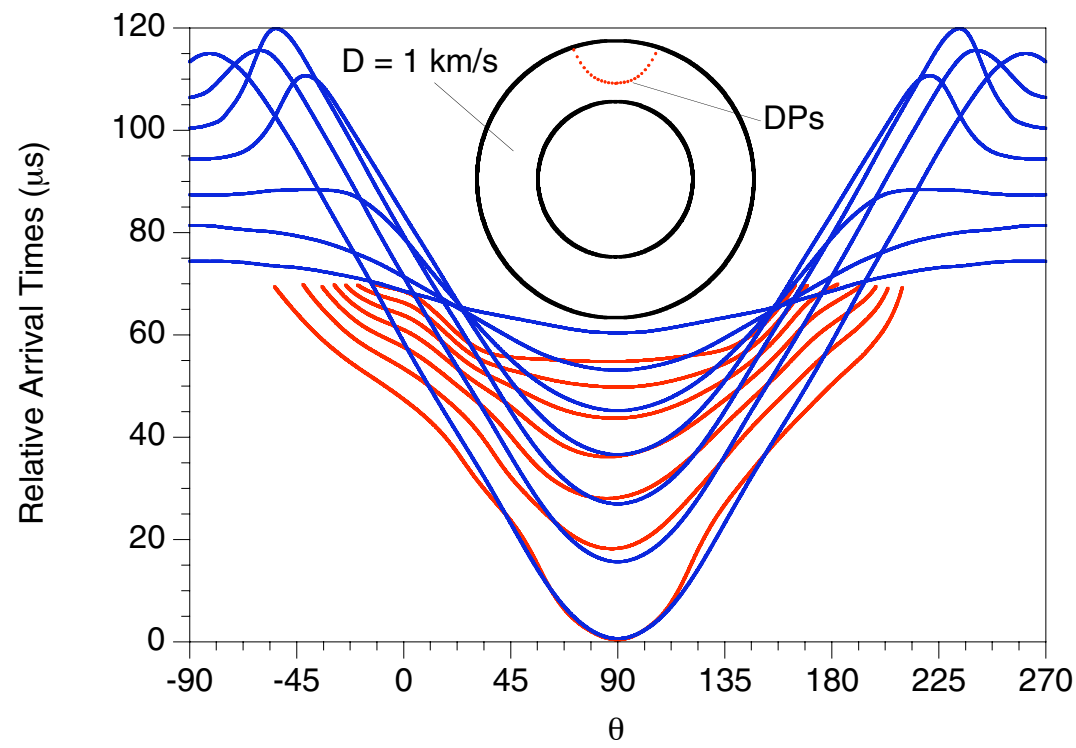

Fig. 8.9. Pin curves for a simulated configuration (blue) with $D=1 \mathrm{~km} / \mathrm{s}$ and $100 \%$ of $E_{P B X-9501}$. Initiation points coincide with the pressure-front locus from a one-detonation point at the outer liner.

These results corroborate, in part, our hypothesis about the radius of curvature of the pressure front discussed above. Thus, it appears that the initiation region was closer to the outer liner than to the inner one; otherwise, the leading edge of the pressure front would not have been sufficiently flat to effect the deformation shown in the photographs of Shot 6 (Fig. 6.2). Here, there is a noticeable improvement in matching the timing when it is 
compared with a one-point initiation near the outer liner, shown in Fig. 8.5. However, neither the late-time behavior nor the change in the slope of the experimental pin curves is captured. In the configuration shown in Fig. 8.10, we have approximated a small bulkburn region by placing 40 detonation points around a small ellipsoidal region located closer to the outer liner. The number and location of these points are rather arbitrary, but the intent was to investigate the effect of initiation size. The propagation velocity is 1 $\mathrm{km} / \mathrm{s}$, and the available energy in the $\mathrm{HE}$ is $100 \%$ of $E_{P B X-9501}$. Notice the slight improvement in matching the timing when it is compared with Fig. 8.9. From the results of these last two cases, we can infer that it is not possible to precisely determine the radial location and the angular extent of the initiation region by relying solely on calculated pincurve data that is based on two-dimensional photographic records.

In Fig. 8.11, we have expanded the initiation region to encircle a wider area, which is reminiscent of a simulated isotherm (Zerkle and Luck 2003; Aviles-Ramos 2004). About 50 initiation points were placed along the contour to approximate a bulk burn. The pin curves are very similar to those of Fig. 8.5, but the agreement with the data is not as good as that for Figs. 8.9 and 8.10. This makes it clear that very similar pin curves can be obtained by using completely different initiation region topologies and locations.

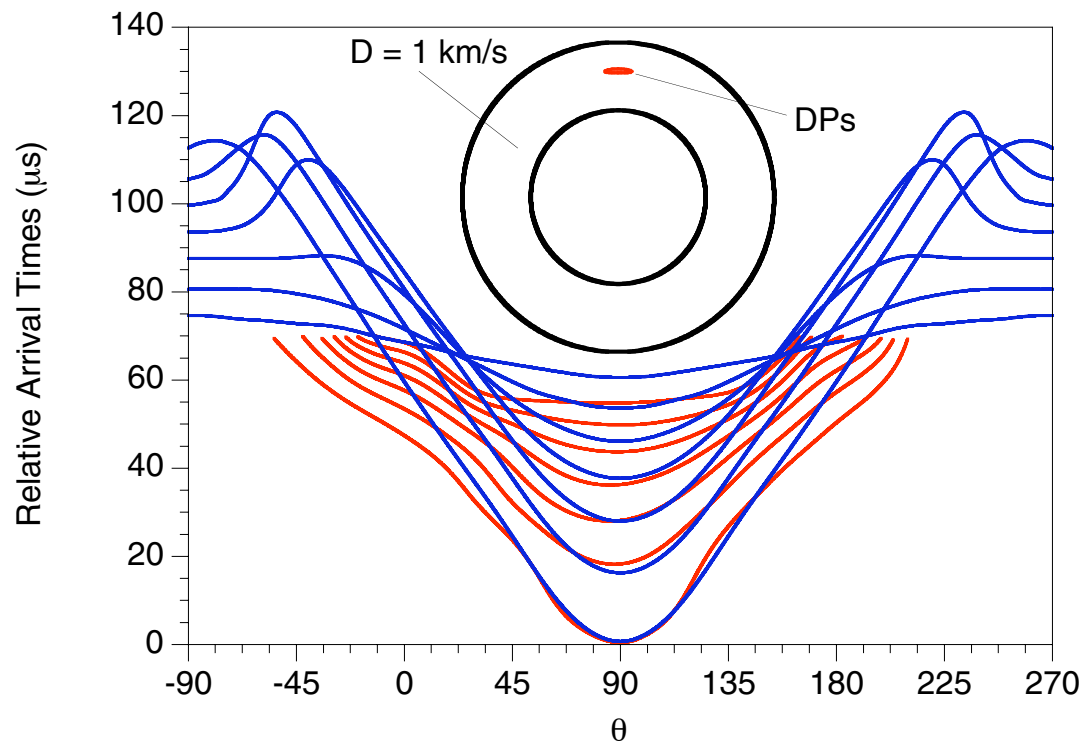

Fig. 8.10. Pin curves for a simulated configuration (blue) with $D=1 \mathrm{~km} / \mathrm{s}$ and $100 \%$ of $E_{P B X-9501}$. Initiation points are on the locus of a small ellipsoid closer to the outer liner. 


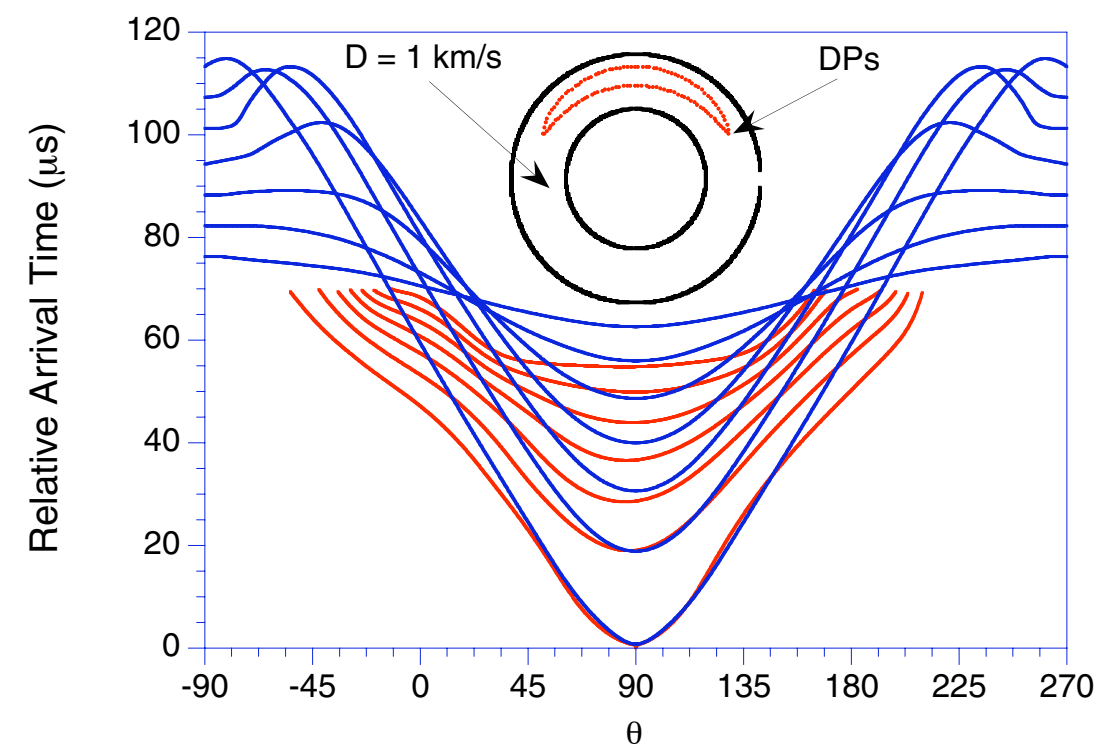

Fig. 8.11. Pin curves for a simulated configuration (blue) with $D=1 \mathrm{~km} / \mathrm{s}$ and $100 \%$ of $E_{P B X-9501}$. Initiation points are on the locus of an ellipsoid that covers a sector larger than $90^{\circ}$.

The case depicted in Fig. 8.12 is identical to the previous one, except the reaction front velocity was increased to $2 \mathrm{~km} / \mathrm{s}$. As shown in previous pin plots, whenever the reacting front velocity is greater than $1 \mathrm{~km} / \mathrm{s}$, the overall agreement declines.

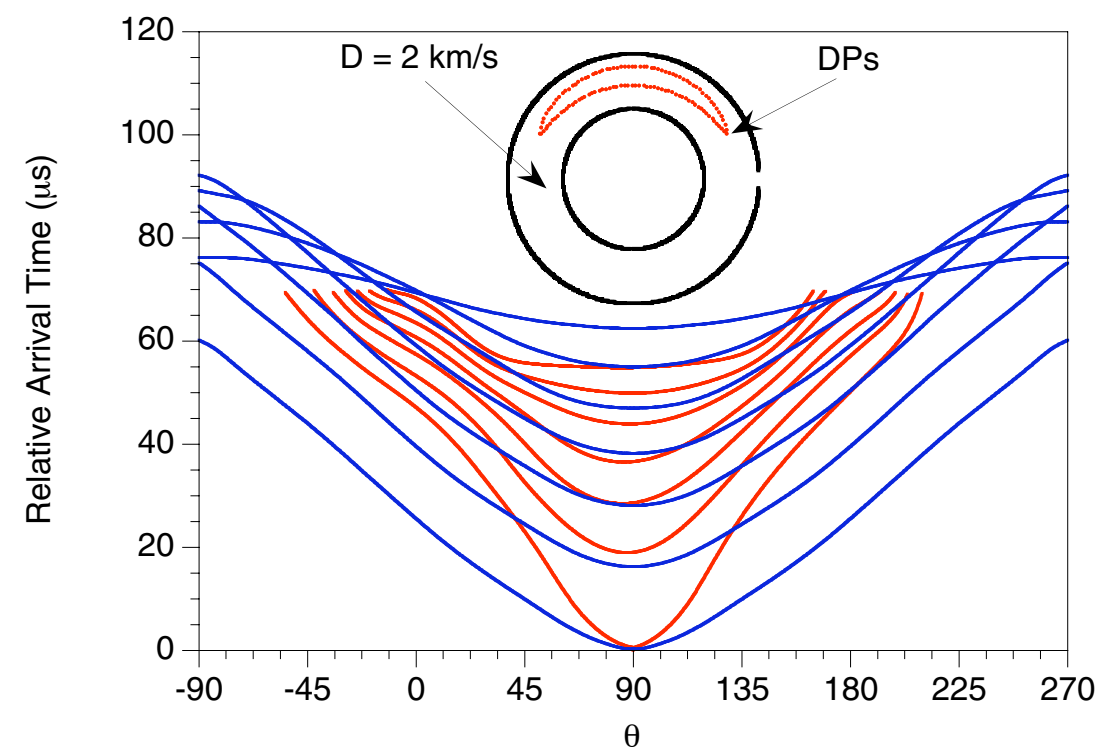

Fig. 8.12. Pin curves for a simulated configuration (blue) with $D=2 \mathrm{~km} / \mathrm{s}$ and $100 \%$ of $E_{P B X-9501}$. Initiation points are on the locus of an ellipsoid that covers a sector larger than $90^{\circ}$.

We have discussed several configurations in which the HE characteristics are identical throughout. In each configuration, we varied the reaction front velocity, the 
available chemical energy upon initiation, and the location and arrangement of the initiation points. It was shown that in some instances, a configuration with a certain reaction front velocity would match the experimental pin curves within a certain angular domain, whereas if a different velocity was used, a match for a different domain would be obtained. This suggests that the propagation velocity of the reactive front through the thermally damaged $\mathrm{HE}$ is nonuniform and thus cannot be accurately represented by a single value, as it can be with pristine HE.

In the following configurations, we have introduced at least two regions within the HE domain that have different propagation velocities. We have also restricted the variation of the reaction front velocity to the plane perpendicular to the assembly axis. In the configuration shown in Fig. 8.13, a crescent-shaped region that covers a sector larger than $270^{\circ}$ has been assigned a velocity of $1 \mathrm{~km} / \mathrm{s}$, and $2 \mathrm{~km} / \mathrm{s}$ has been assigned everywhere else (outside the crescent-shaped cylinder). Both regions are capable of releasing $100 \%$ of $E_{P B X-9501}$, and the initiation point is located in the middle of the crescent. As in other configurations in which a $2 \mathrm{~km} / \mathrm{s}$ reaction front velocity was used (Figs. 8.8 and 8.12), the only agreement was between the slopes of the pin curves for $30^{\circ}>\theta>150^{\circ}$. Essentially, the same behavior is found when a series of initiation points (instead of a single point) are placed inside the crescent-shaped region, around the locus of a stretched ellipsoid that covers a sector larger than $90^{\circ}$, as shown in Fig. 8.14. From these two HE configurations, it can be inferred that there is no significant correlation between temperature distribution and reacting front-propagation velocity after thermal runaway.

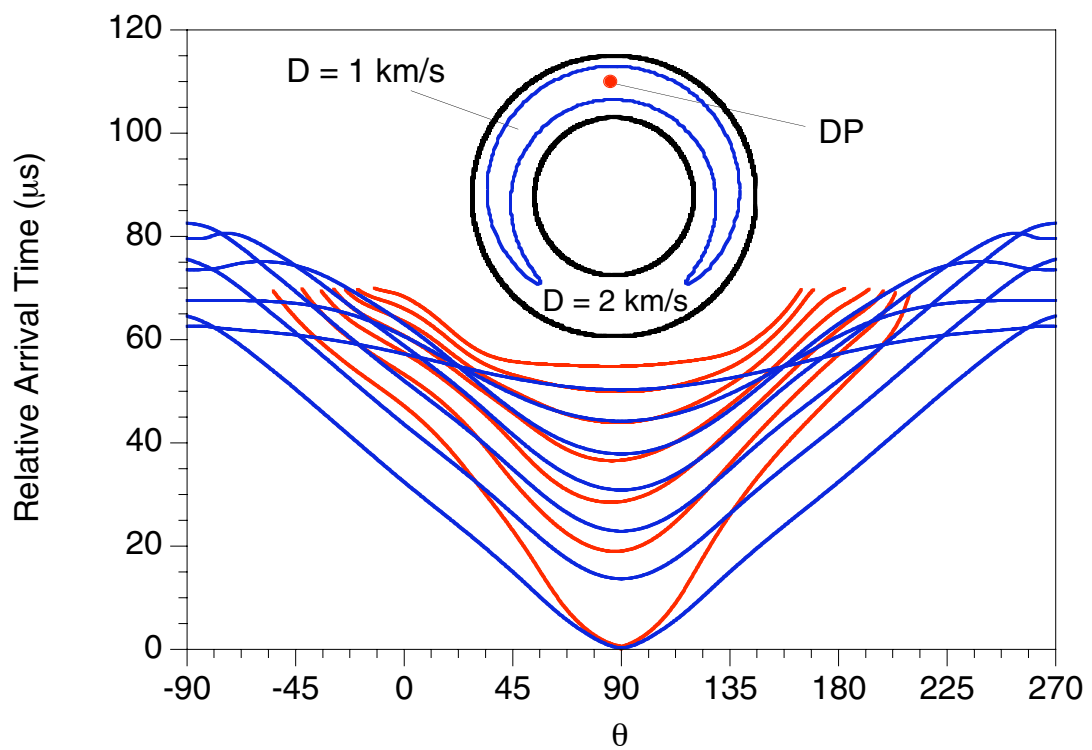

Fig. 8.13. Pin curves for a simulated configuration (blue) with $D=1$ and $2 \mathrm{~km} / \mathrm{s}$ (inside and outside, respectively, of a crescent-shaped region that covers a sector larger than $270^{\circ}$ ) and $100 \%$ of $E_{P B X-9501}$. Initiation point is at midpoint. 


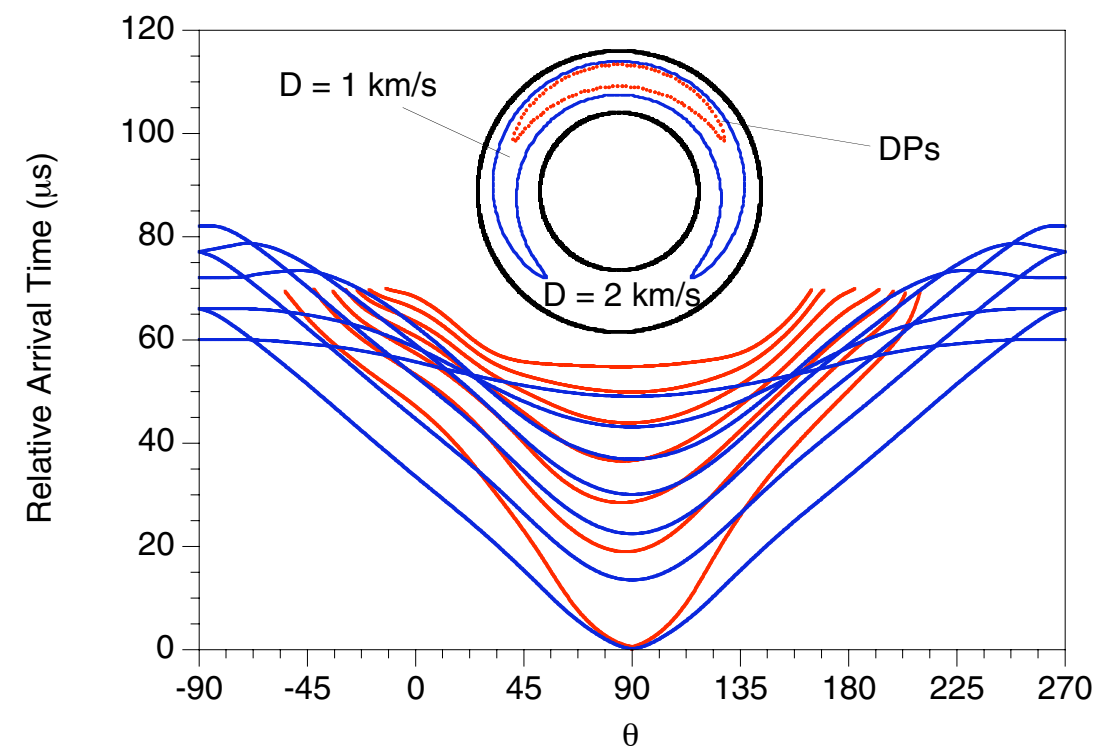

Fig. 8.14. Pin curves for a simulated configuration (blue) with $D=1$ and $2 \mathrm{~km} / \mathrm{s}$ (inside and outside, respectively, of a crescent-shaped region) and $100 \%$ of $E_{P B X-9501}$. Initiation points are around a stretched ellipsoid that covers a sector larger than $90^{\circ}$.

In the configuration shown in Fig. 8.15, the HE region consists of two hemicylinders of degraded PBX-9501, with reaction front velocities of 1 and $2 \mathrm{~km} / \mathrm{s}$, respectively. The JWL parameters were chosen so that both regions of HE would be capable of releasing $100 \%$ of $E_{P B X-9501}$. The burn front is initiated at single point near the outer liner, within the region corresponding to a reaction front velocity of $1 \mathrm{~km} / \mathrm{s}$. The pin curves for this configuration match the experimental curves for Shot 6 much better than the pin curves did for the configurations in Figs. 8.13 and 8.14. Notice that the change in slope is captured on the first pin curve, although the timing diverges beyond the third curve. In Fig. 8.16, we have replaced the lower hemicylinder with pristine PBX-9501. In this configuration, the timing is closer to that of the experimental pin curves, but the curvature diverges drastically beyond the sector $30^{\circ}>\theta>150^{\circ}$. 


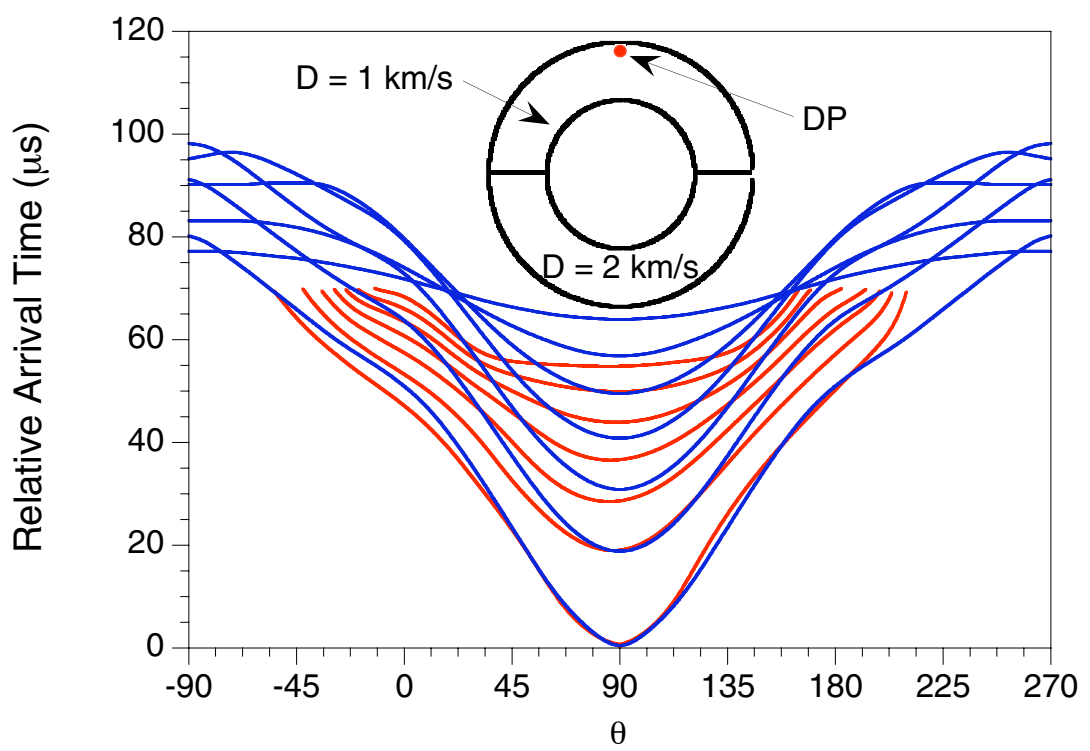

Fig. 8.15. Pin curves for a simulated configuration (blue). One-half of the HE has $\mathrm{D}=1 \mathrm{~km} / \mathrm{s}$, and the other half has $2 \mathrm{~km} / \mathrm{s}$; both halves have $100 \%$ of $E_{P B X-9501}$. Initiation point is at the outer liner.

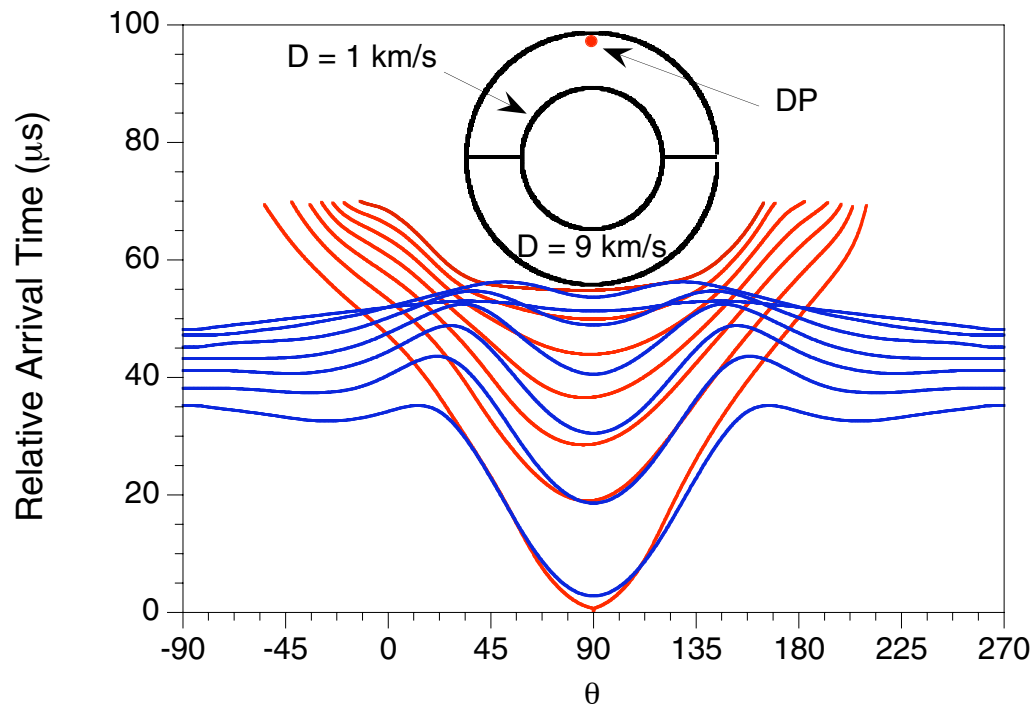

Fig. 8.16. Pin curves for a simulated configuration (blue). One-half of the HE has $\mathrm{D}=1 \mathrm{~km} / \mathrm{s}$, and the other half has $9 \mathrm{~km} / \mathrm{s}$; both halves have $100 \%$ of $E_{P B X-9501}$. Initiation point is at the outer liner.

In Fig. 8.17, we placed 30 initiation points along the isobar whose leading edge was located $1.16 \mathrm{~cm}$ from the inner liner (see also Fig. 8.9). The hemicylinders of HE have the same characteristics as those in Fig. 8.15. If we look at the first pin curves in the sector $30^{\circ}<\theta<150^{\circ}$, we see that this configuration has brought the agreement between experiment and simulation closer than any of the previous configurations have. But Fig. 8.18 shows that reducing the extent of the region having a front propagation velocity of 1 
$\mathrm{km} / \mathrm{s}$ to one-quarter of the HE domain causes the agreement between pin curves to depart significantly from the data. To examine the effect of progressively increasing the detonation velocity from 1 to $9 \mathrm{~km} / \mathrm{s}$, we replaced the lower quarter of the HE with pristine PBX-9501, shown in Fig. 8.19. Only in the central region of the diagram do we see some agreement with the experimental pin curves. These results suggest that on average, the reaction front in Shot 6 propagated at about $1 \mathrm{~km} / \mathrm{s}$ through about half of the $\mathrm{HE}$ domain and did not reach the high velocity of the pristine HE during the first $100 \mu \mathrm{s}$.

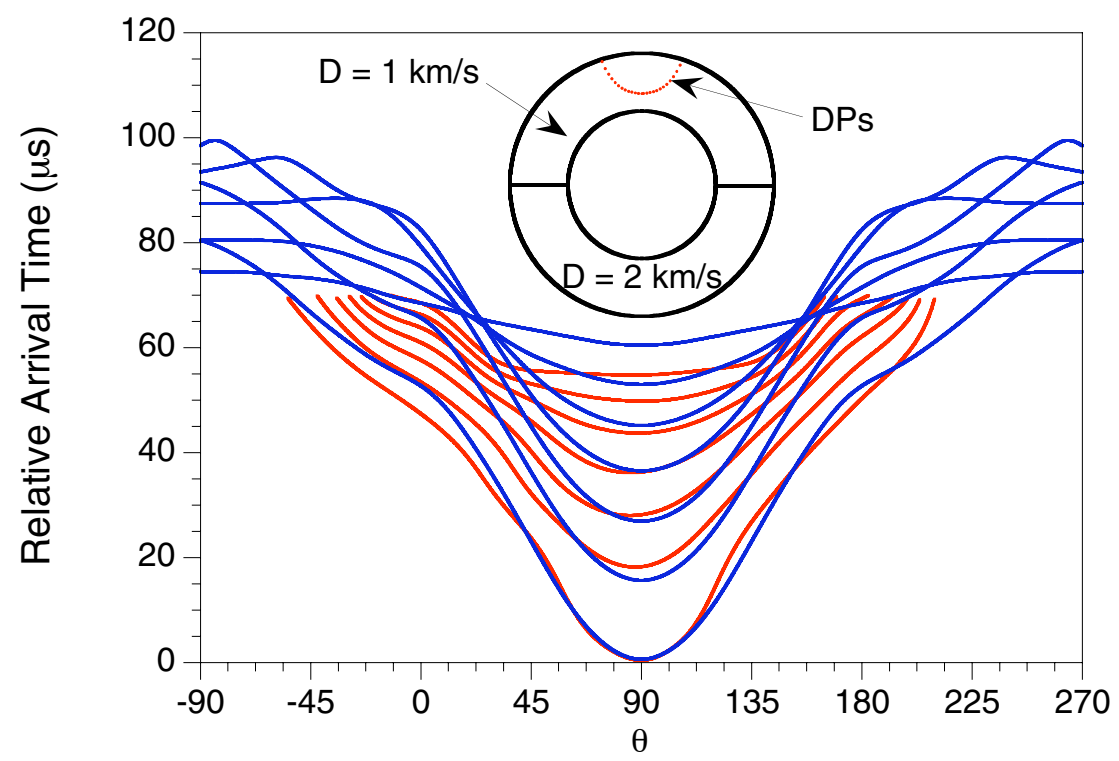

Fig. 8.17. Pin curves for a simulated configuration (blue). One-half of the HE has $\mathrm{D}=1 \mathrm{~km} / \mathrm{s}$, and the other half has $2 \mathrm{~km} / \mathrm{s}$; both halves have $100 \%$ of $E_{P B X-9501}$. Initiation points coincide with the pressure front locus from a one-initiation point located near the outer liner. 


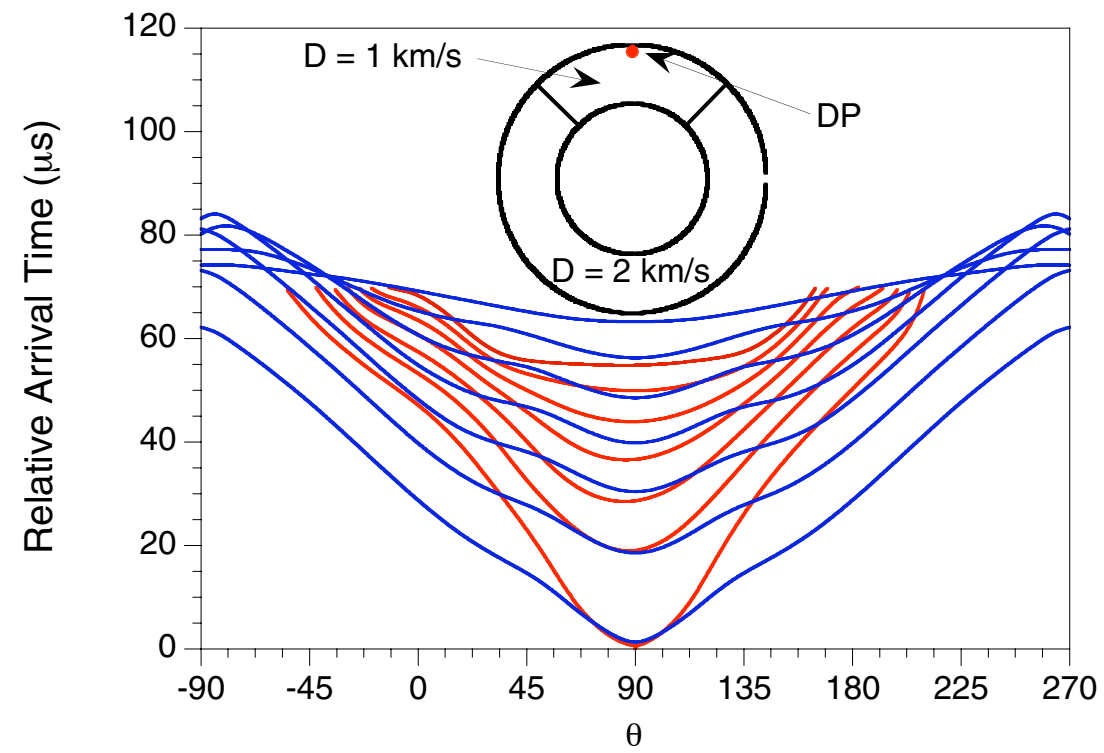

Fig. 8.18. Pin curves for a simulated configuration (blue). One-quarter of the $\mathrm{HE}$ has $\mathrm{D}=1 \mathrm{~km} / \mathrm{s}$, and the rest has $2 \mathrm{~km} / \mathrm{s}$; both halves have $100 \%$ of $E_{P B X-9501}$. Initiation point is located at the outer liner.

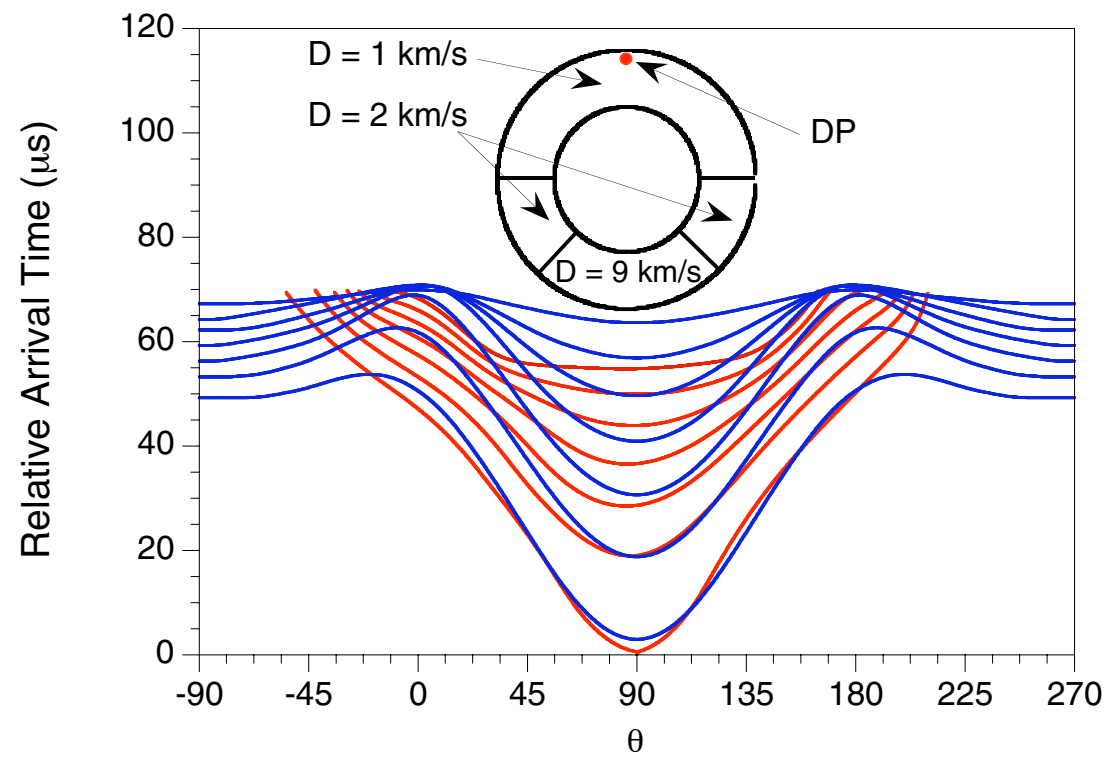

Fig. 8.19. Pin curves for a simulated configuration (blue). One-half of the HE has $D=1 \mathrm{~km} / \mathrm{s}$, one-quarter has $2 \mathrm{~km} / \mathrm{s}$, and the rest has $9 \mathrm{~km} / \mathrm{s}$; all are with $100 \%$ of $E_{P B X-9501}$. Initiation point is located at the outer liner.

In the configuration shown in Fig. 8.20, the HE region consists of two hemicylinders of degraded PBX-9501 with propagation front velocities of 1 and $2 \mathrm{~km} / \mathrm{s}$, respectively. The JWL parameters were chosen so that both regions of HE would be capable of 
releasing $100 \%$ of $E_{P B X-9501}$. Initiation occurs along a small ellipsoid similar to a simulated isotherm within the region corresponding to a detonation velocity of $1 \mathrm{~km} / \mathrm{s}$. Since this configuration, together with those shown in Figs. 8.15 and 8.17, turned out to yield the closest agreement with experimental data, simulations were performed using both the $0.56 \times 10^{6}$ and the $4.5 \times 10^{6}$-cell models (results shown in Fig. 8.20 correspond to the finer grid simulations). A fine grid is necessary to spatially resolve the shock wave interactions in the inner liner (see Section 8.2). Notice that the numerical simulation approximately captures the change in slope for the first pin curve at $\theta \approx 15^{\circ}$, and the relative timing of the first four curves at $\theta=90^{\circ}$ is in excellent agreement with the data. However, the slope change for later times occurs farther away from $\theta=90^{\circ}$ in the simulation than it does in the data. Overall, the configuration shown in Fig. 8.17 is marginally better because the timing of the last pin curve $\left(r / R_{i}=0.125\right)$ is closer to the pin curve for Shot 6. In terms of compression levels, the dynamic response of the inner liner to the HE configurations is essentially the same in Figs. 8.15, 8.17, and 8.20. An important finding that surfaces from the results we computed for these three configurations is that initiation probably started at a small, localized region close to the outer liner.

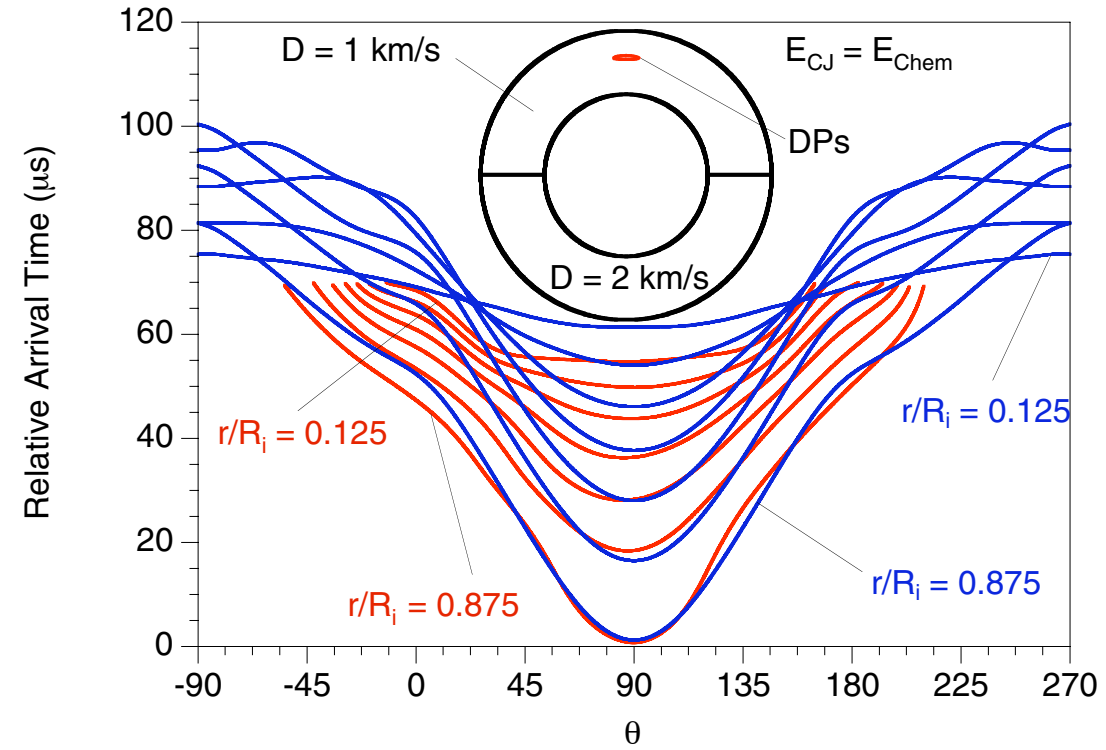

Fig. 8.20. Pin curves for a simulated configuration (blue). One-half of the HE has $\mathrm{D}=1 \mathrm{~km} / \mathrm{s}$, and the other half has $2 \mathrm{~km} / \mathrm{s}$; both halves have $100 \%$ of $E_{P B X-9501}$. Initiation points are on the locus of a small ellipsoid closer to the outer liner.

\subsection{Inner-Liner Pressure and Compression Distributions}

The asymmetry of the collapse, evidenced qualitatively by the topology of the collapsing contours and quantitatively by our relative measure of geometric symmetry (i.e., pin plots), is a manifestation of the nonuniform distribution of the stress tensor around the inner liner at every radial location. This, in turn, leads to a nonuniform 
azimuthal compression, which can be quantified by the fractional compression ratio $\mu$ defined by $\mu(r, \theta, z, t)=\frac{\rho(r, \theta, z, t)}{\rho_{0}}-1$,

where $\rho(r, \theta, z, t)$ is the instantaneous local density of the metal, and $\rho_{0}$ is the initial density.

In what follows, we computed the pressure and the density in the midplane of the inner liner at an initial constant radial location $(r=4.13 \mathrm{~cm}, z=0)$, as a function of angle $\theta$ and time $t$. Models with a grid size of $0.1 \mathrm{~cm}$ were used in these simulations $\left(4.5 \times 10^{6}\right.$ cells) to capture the shock fronts through the inner liner. Initially, this region traces a circle, but as the inner liner collapses, this "material curve" deforms. Computationally, we are following this material region of fixed identity.

Figures 8.21( $\mathrm{a}$ and $\mathrm{b}$ ) show the azimuthal pressure distribution at different times resulting from a small initiation region and two hemicylinders of HE reaction front propagation velocities of 1 and $2 \mathrm{~km} / \mathrm{s}$ (Fig. 8.20). In this configuration, the peak pressure is $67.6 \mathrm{GPa}$ and occurs at about $155 \mu \mathrm{s}$ at $\theta=90^{\circ}$ (the same angular location as the center of the initiation region) as a result of the collision of the opposite sides of the inner liner. Not only is the angular pressure distribution highly nonuniform around the inner liner, but there are also instances in which tension and compression are instantaneously exerted at different angles along a constant radial location. Clearly, the peculiar shape of the collapsing contour is a direct consequence of nonuniformities in the stress tensor field within the inner liner, which can be traced to the dynamic deformation of metal resulting from localized initiation. Our results show that even after the inner liner has completely collapsed into itself, the azimuthal pressure distributions remain asymmetric. Similar asymmetric distributions are observed for the compression ratio. Figures 8.22(a and b) show the compressions at the same times for which the pressures were computed. As expected, the compression curves follow a trend similar to the pressure curves (the same can be said of the internal energy curves, which we also computed but do not show in this report). Thus, it is not difficult to infer that the behavior of other thermodynamic state variables would also vary continuously in time and space. 


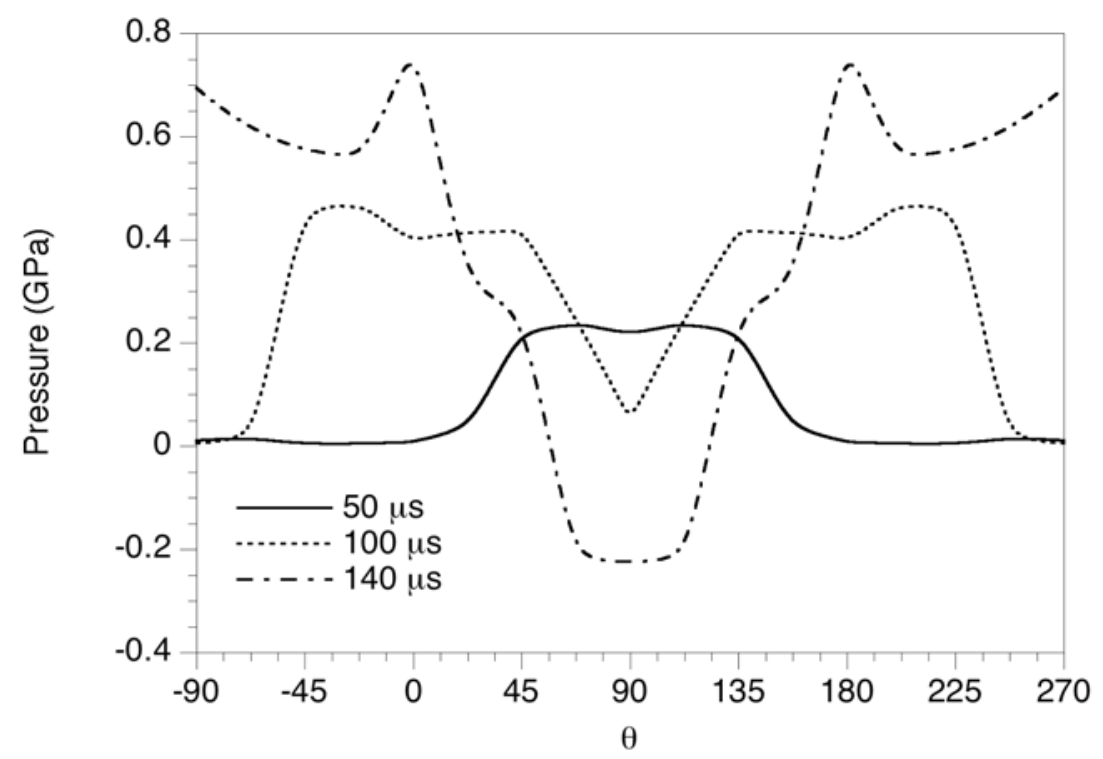

Fig. 8.21a. Azimuthal pressure distribution in the inner liner at three different times before complete collapse. One-half of the HE has $D=1 \mathrm{~km} / \mathrm{s}$ and the other half has $2 \mathrm{~km} / \mathrm{s}$; both halves have $100 \%$ of $E_{P B X-9501}$. Initiation points are on the locus of a small ellipsoid close to the outer liner.

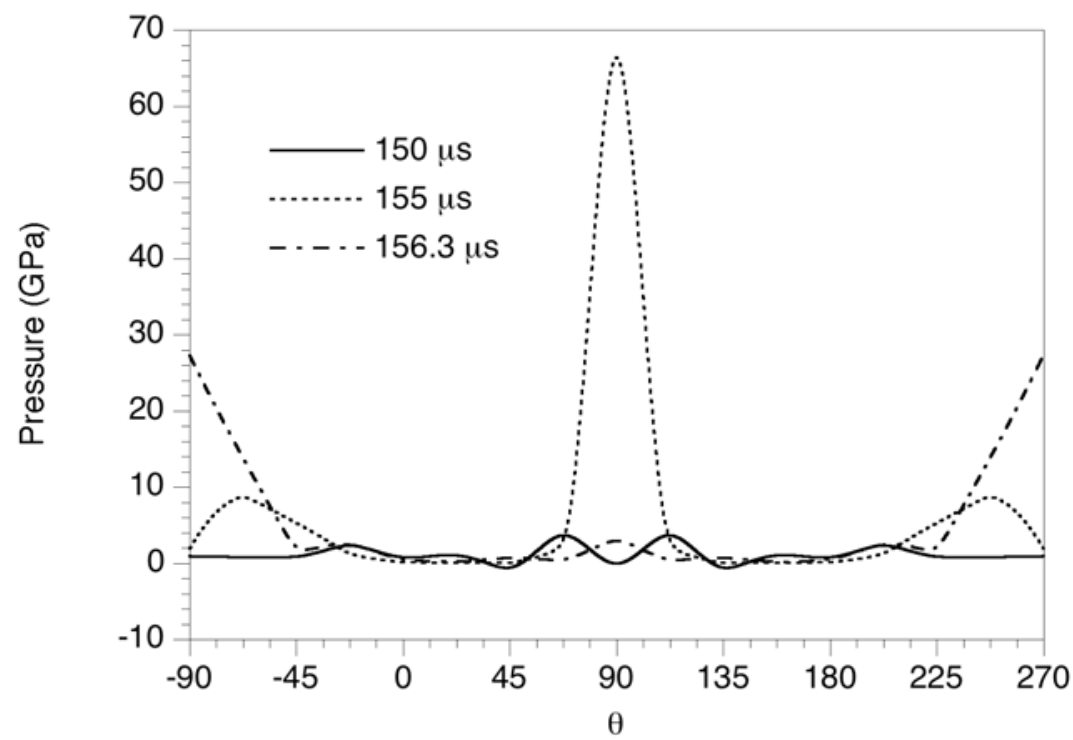

Fig. 8.21b. Azimuthal pressure distribution in the inner liner at three different times near complete collapse. One-half of the HE has $D=1 \mathrm{~km} / \mathrm{s}$, and the other half has $2 \mathrm{~km} / \mathrm{s}$; both halves have $100 \%$ of $E_{P B X-9501}$. Initiation points are on the locus of a small ellipsoid close to the outer liner.

As stated earlier, it is physically impossible for an asymmetric initiation within the $\mathrm{HE}$, as it occurred in Shot 6, to evolve into a symmetric collapse at a later time, regardless of the characteristics of the HE. This can be understood by comparing the 
previous configurations (Figs. 8.21 and 8.22) with simulations with pristine PBX-9501. A striking difference between these two cases is the angular location of the highest achievable pressure. For the degraded HE simulations, the peak pressure is $67.6 \mathrm{GPa}$ and occurs at $\theta=90^{\circ}$, whereas for the pristine $\mathrm{HE}$, the peak pressure is $129.6 \mathrm{GPa}$ and occurs at $\theta=-90^{\circ}$ (see Fig. 8.23 b). In the pristine case, the high CJ pressure detonation fronts originating at $\theta=90^{\circ}$ travel in opposite directions around the assembly and collide, creating a higher pressure at $\theta=-90^{\circ}$ that locally accelerates the liner at a higher velocity than that at $\theta=90^{\circ}$. This effect is not observed in the degraded HE configuration because the maximum pressure is considerably lower than the pressure of the pristine HE. The peak pressures take place when the inner liner has fully collapsed into itself, that is, when opposite ends of the liner at $\theta=90^{\circ}$ and $\theta=-90^{\circ}$ have collided. The collision times for the degraded and pristine HE cases are 155 and $34.7 \mu$ s, respectively.

It is evident from these two simulations that there is no general trend of behavior for pressure, compression, or other thermodynamic variables applicable to an annular geometry when initiation occurs at a localized region (i.e., asymmetric initiation). The azimuthal distribution of these variables is the result of multiple interactions of shock and rarefaction waves ringing through the inner liner, which are strongly dependent on geometry, size, HE characteristics, and initiation location.

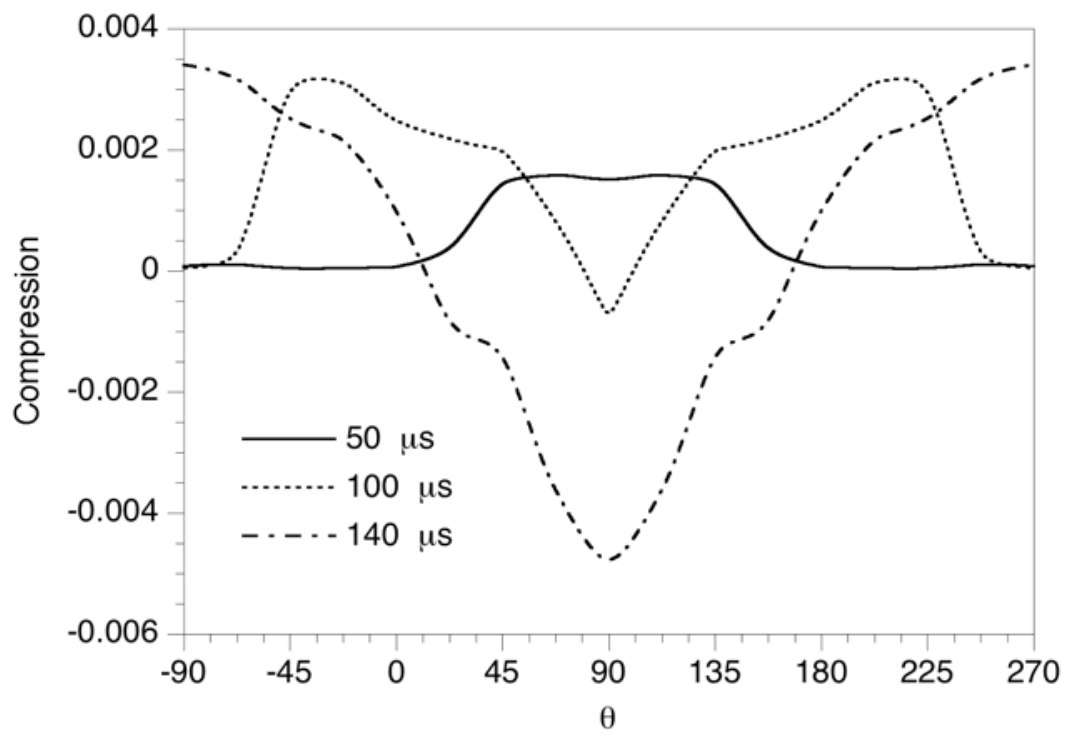

Fig. 8.22a. Azimuthal compression distribution in the inner liner at three different times before complete collapse. One-half of the HE has $D=1 \mathrm{~km} / \mathrm{s}$ and the other half has $2 \mathrm{~km} / \mathrm{s}$; both halves have $100 \%$ of $E_{P B X-9501}$. Initiation points are on the locus of a small ellipsoid close to the outer liner. 


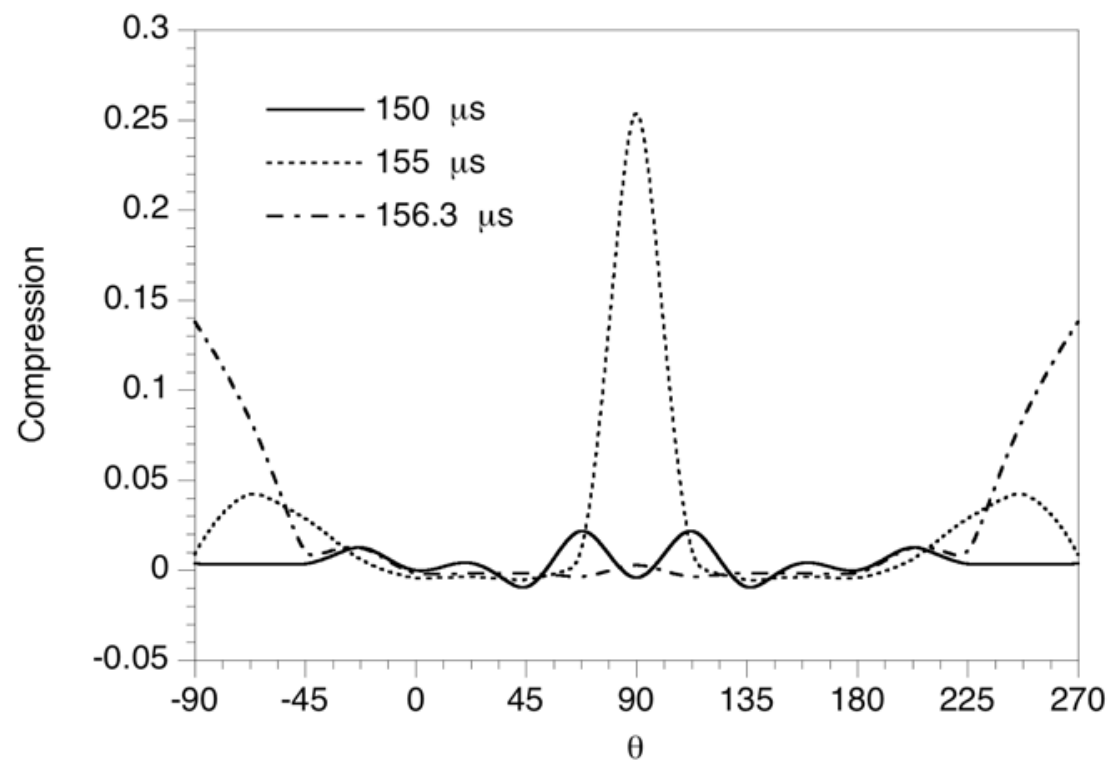

Fig. 8.22b. Azimuthal compression distribution in the inner liner at three different times near complete collapse. One-half of the HE has $D=1 \mathrm{~km} / \mathrm{s}$, and the other has $2 \mathrm{~km} / \mathrm{s}$; both halves have $100 \%$ of $E_{P B X-9501}$. Initiation points are on the locus of a small ellipsoid closer to the outer liner.

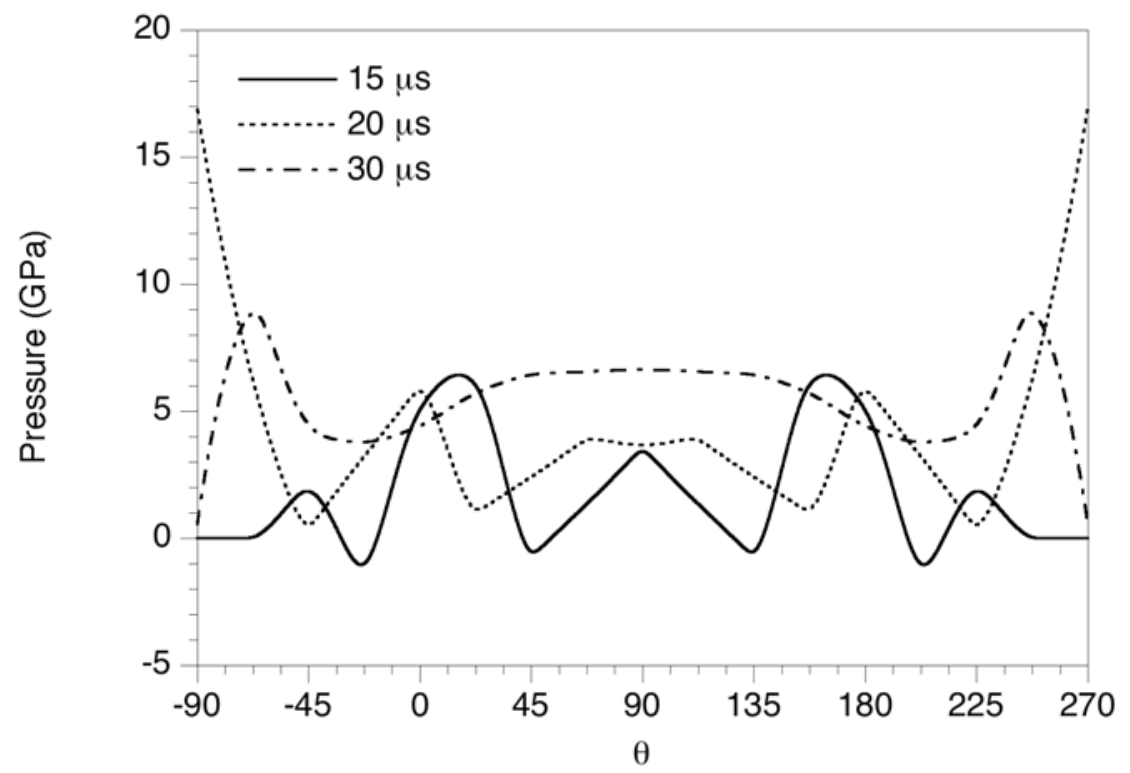

Fig. 8.23a. Azimuthal pressure distribution in the inner liner at three different times before complete collapse. The entire HE is pristine PBX-9501 ( $D=9 \mathrm{~km} / \mathrm{s}$ ), and the detonation point is located near the outer liner. 


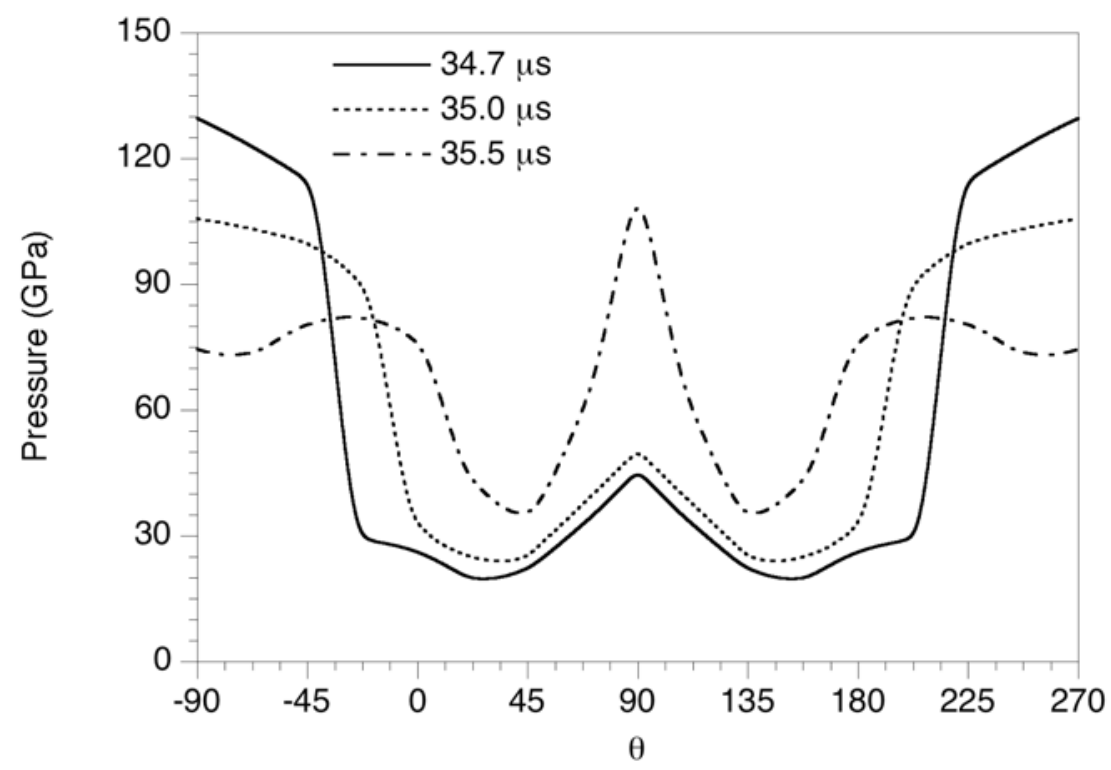

Fig. 8.23b. Azimuthal pressure distribution in the inner liner at three different times near complete collapse. The entire HE is pristine PBX-9501 $(D=9 \mathrm{~km} / \mathrm{s})$, and the detonation point is located near the outer liner.

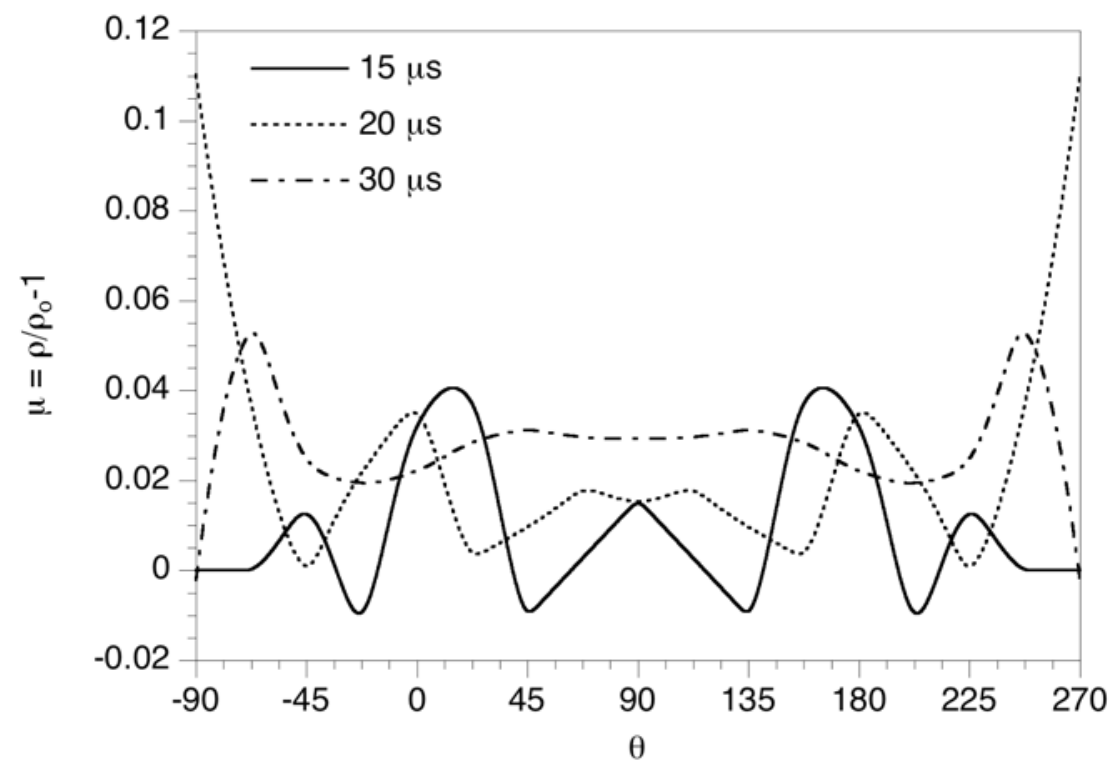

Fig. 8.24a. Azimuthal compression distribution in the inner liner at three different times before complete collapse. The entire HE is pristine PBX-9501 ( $D=9 \mathrm{~km} / \mathrm{s})$, and the detonation point is located near the outer liner. 


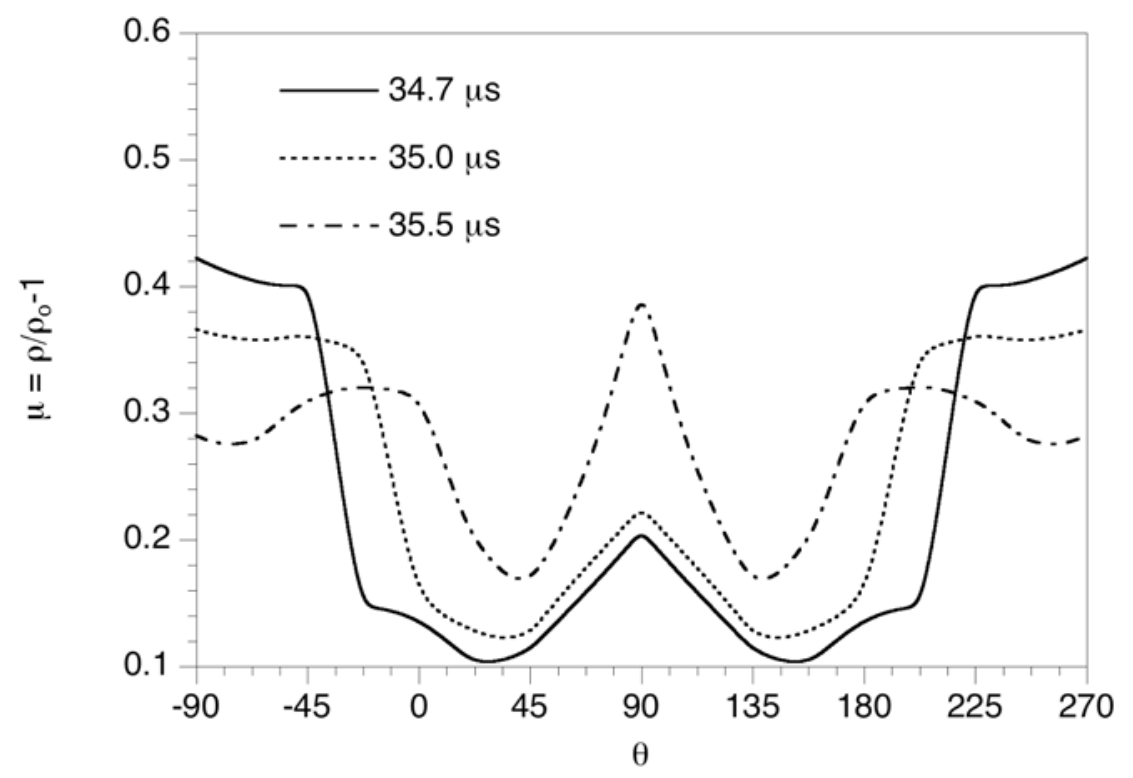

Fig. 8.24b. Azimuthal compression distribution in the inner liner at three different times near complete collapse. The entire HE is pristine PBX-9501 ( $D=9 \mathrm{~km} / \mathrm{s}$ ), and the detonation point is located near the outer liner.

\subsection{Inner-Liner Collapse}

We selected the configuration whose pin curves best agreed with the data and then compared the collapsing inner-liner simulation with the photographs taken in Shot 6 (Fig. 6.2). This configuration consisted of two hemicylinders of degraded PBX-9501 with detonation velocities of 1 and $2 \mathrm{~km} / \mathrm{s}$, respectively, and with JWL parameters that allowed both regions of the $\mathrm{HE}$ to be capable of releasing $100 \%$ of $E_{P B X-9501}$. Initiation points were placed around the locus of a small ellipsoid close to the outer liner, within the region corresponding to a detonation velocity of $1 \mathrm{~km} / \mathrm{s}$ (Fig. 8.20). In the simulated images, the point of view is along the axial direction through the entire model so that one-to-one comparisons with the photographs can be made. Figure 8.25 shows a comparison between the photographs of the inner-liner collapse contours in Shot 6 and the simulations. In these images, time zero corresponds to the first photograph recorded at $30 \mu s$, that is, the instant when one of the trigger wires was first contacted by the imploding liner. In the simulated images, time zero is chosen as the frame whose contour matches the first photograph. We remind the reader that the experimental zero is not the time at which the runaway reaction started. Strain-gage data suggest that the thermal reaction might have started about $40 \mu$ s before the first contact wire was triggered.

Although the simulated images lag behind the actual photographs, the topology of the collapsing liner shows remarkable agreement. It is interesting to note that the peculiar "lima bean" shape of the contour cannot be reproduced using simulated HEs with detonation velocities higher than $2 \mathrm{~km} / \mathrm{s}$. 


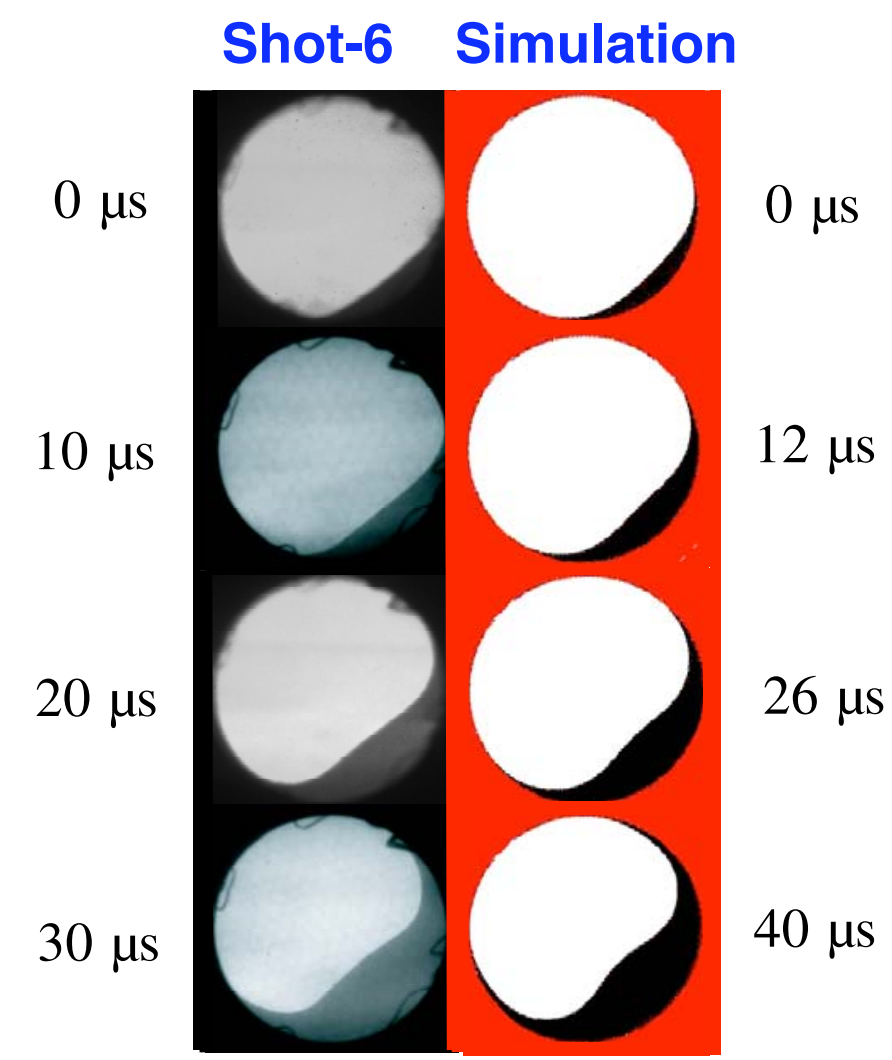

Fig. 8.25. Comparison between the first four photographs of the inner-liner collapse contours in Shot 6 and those from our simulations.

Finally, Fig. 8.26a shows the dimensionless radial displacement of the FMP, as a function of time, obtained from the high-speed photographs for Shot 6 (Fig. 6.2). For comparison, we also show the results from the simulations for the one-point detonation of pristine PBX-9501 (discussed in Section 5) as well as the deflagration of cooked PBX9501, using the approximate deflagration EOS with a reaction velocity of $1 \mathrm{~km} / \mathrm{s}$ (Section 7.1) and one initiation point close to the outer liner, at the midplane of the assembly. The experimental zero time is defined as the time at which the FMP reached a resistance wire located $1 \mathrm{~mm}$ from the inner liner. For pristine PBX-9501, our simulations show that the inner-liner collapse is predicted to occur at about $27 \mu \mathrm{s}$, whereas a slower collapse is observed in Shot 6 and in the calculated configuration for degraded PBX-9501. As noted earlier, simulations with the approximate deflagration EOS tend to underestimate the rate of collapse of the inner liner but capture the trend and the essential features of the deformation topology of the liner. The velocity of the FMP interpolated from the test data for Shot 6 and the simulated velocity calculated using the approximate deflagration EOS are shown in Fig. 8.26b. Simulations of the deflagration of PBX-9501 progressively underestimate the interpolated test results as a function of time. 


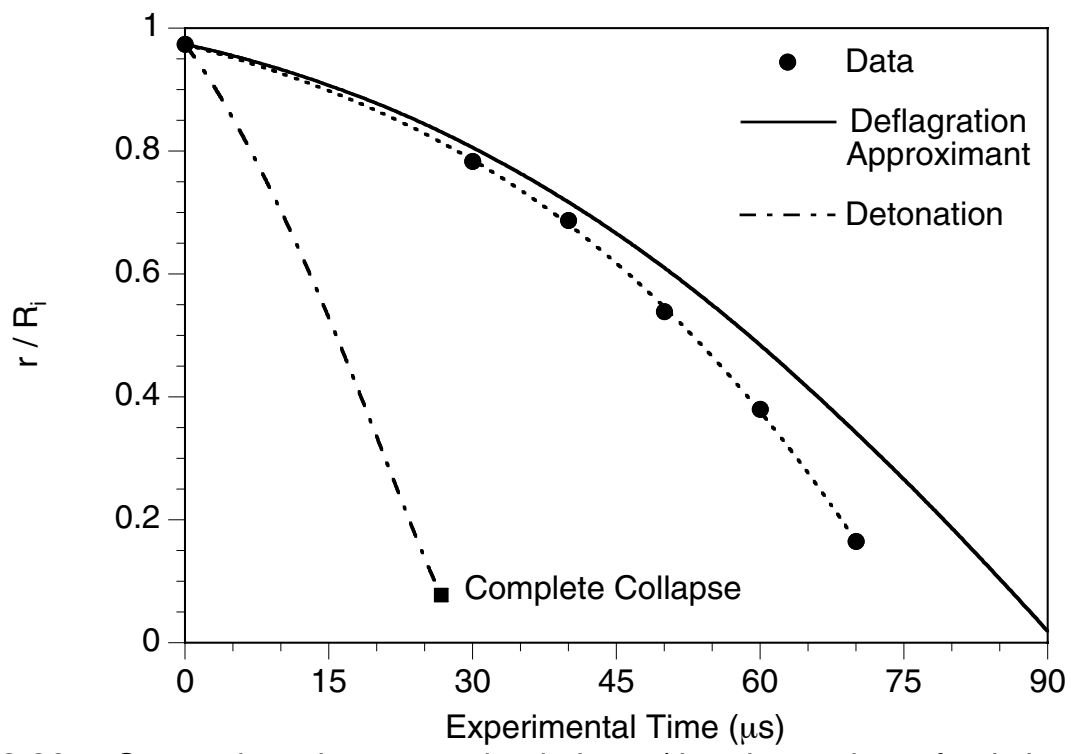

Fig. 8.26a. Comparison between simulations (the detonation of pristine PBX9501 and deflagration of cooked PBX-9501) and Shot 6 data for the inner-liner FMP.

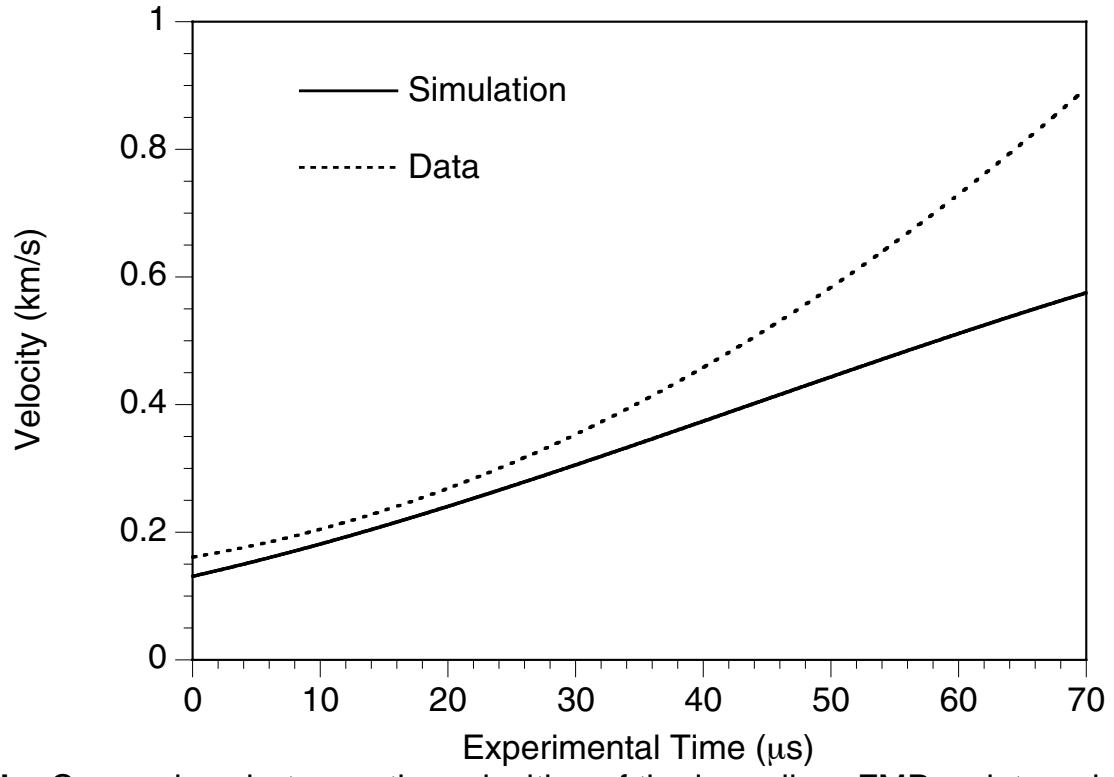

Figure 8.26b. Comparison between the velocities of the inner liner FMP as interpolated from Shot 6 data and from simulations for the deflagration of PBX-9501. 


\section{Outer-Liner and End-Cap Deformation: Numerical Simulation Results}

In addition to the data obtained by high-speed photography, useful data for Shot 6 were obtained from the VISAR (aimed at one end cap), accelerometers (on the outer liner), and RWs (around the outer liner). We compared these data with our PAGOSA simulations for the deflagration of PBX-9501. To develop our approximate deflagration EOS for Shot 6, several simulations were conducted to determine which input parameters provided the best agreement with the experimental results. Initially, we focused on reproducing the inner-liner behavior from the high-speed photographs. As discussed in Chapter 8 , the input parameters we were interested in included the number and location of initiation points, the EOS for the cooked PBX-9501, the resolution of the mesh, and the possibility of having vents that were caused by the failure of the casing confining the energetic material and decomposition gases. From the calculations, we determined that one initiation point located close to the outer edge of the energetic material at the midplane of the assembly resulted in good agreement with the observed behavior in Shot 6 . A mesh size of $0.2 \mathrm{~cm}$, which gave us $5.6 \times 10^{5}$ cells, was selected to model the outer-liner and end-cap behavior (the PAGOSA input file for Shot 6 is included in the Appendix C).

The accelerometer and RW data provide information about the behavior of the outer liner of the LSAC assembly. In Shot 6, these instruments indicate that the outer wall of the casing starts moving approximately $45 \mu$ s after the beginning of the thermal runaway reaction, and the outer wall begins to expand approximately $20 \mu \mathrm{s}$ after this initial movement.

\subsection{Accelerometer Data}

In Fig. 2.7, the accelerometer data from Shot 6 indicate that the signal from the ignition reaction first reaches accelerometer 2 , which is located at an angular location of $210^{\circ}$, and that the signal peaks at approximately $55 \mu \mathrm{s}$. Accelerometer 3 , located at an angular location of $75^{\circ}$, peaks at about $79 \mu \mathrm{s}$, and finally, accelerometer 1 , located at an angular location of $150^{\circ}$, peaks at approximately $84 \mu \mathrm{s}$. The accelerometer data from Shot 6 provide information only about the time of arrival of a signal to the specific location of the accelerometer. In PAGOSA, the signal traveling through the copper outer liner is modeled by calculating the time when the pressure peaks at three Lagrangian tracer particles that are at the specified locations for the accelerometers. The results from the simulations for the pressure behavior at the accelerometer locations are shown in Figure 9.1. 


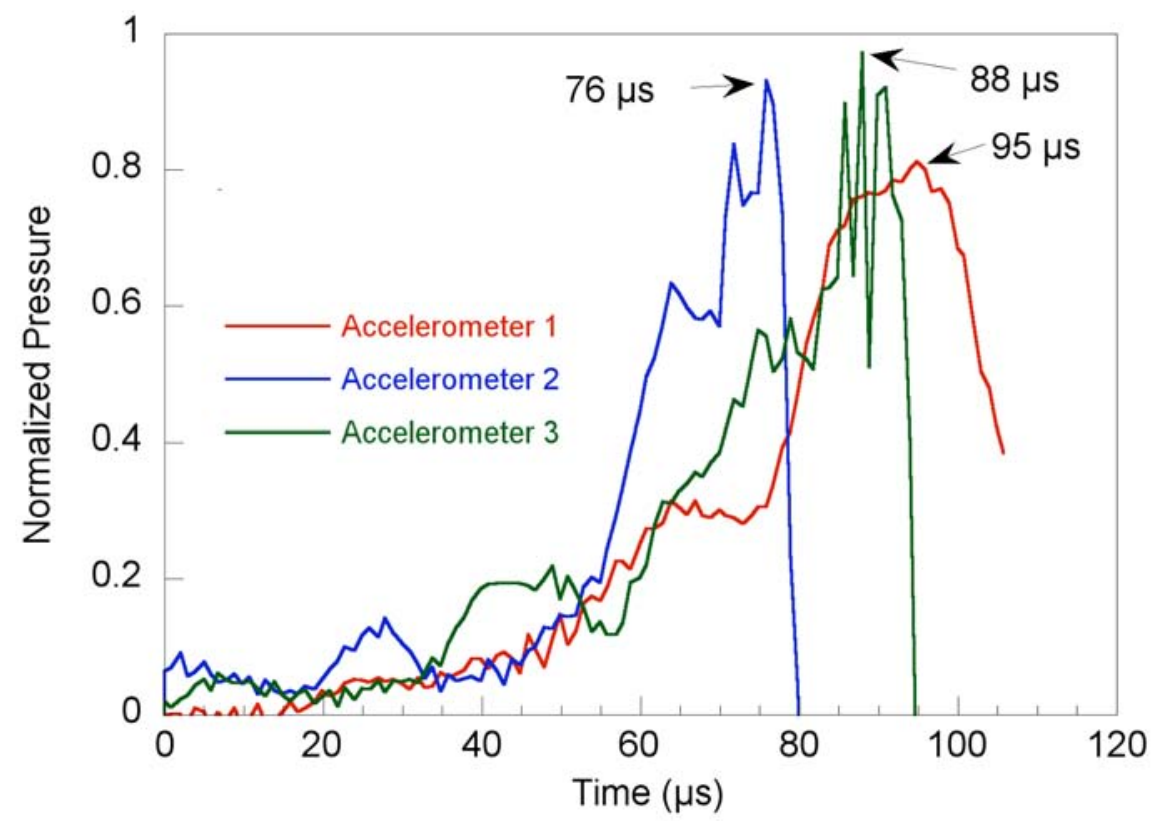

Fig. 9.1. PAGOSA simulations for the pressure behavior at the accelerometer locations in Shot 6.

In Fig. 9.1, the normalized pressure peaks at $76 \mu$ s at accelerometer 2, at $88 \mu$ s at accelerometer 3, and at $95 \mu \mathrm{s}$ at accelerometer 1 . The comparison between the experimental and computational results of the accelerometer behavior in Shot 6 is presented in Table 9.1.

Table 9.1. Comparison between Simulations and Shot 6 Arrival Time

\begin{tabular}{|c|c|c|c|c|}
\hline \multirow{2}{*}{ Accelerometer } & \multirow{2}{*}{$\begin{array}{l}\text { Angular } \\
\text { Location }\end{array}$} & \multicolumn{2}{|c|}{ Arrival Time ( $\mu \mathrm{s})$} & \multirow{2}{*}{$\begin{array}{c}\text { Relative Error } \\
(\%)\end{array}$} \\
\hline & & Test & Code & \\
\hline 1 & $150^{\circ}$ & 84 & 95 & 13 \\
\hline 2 & $210^{\circ}$ & 55 & 76 & 38 \\
\hline 3 & $75^{\circ}$ & 79 & 88 & 11 \\
\hline
\end{tabular}

Simulations underestimate the arrival time detected by the accelerometers by an average relative error of $21 \%$. By considering that the ignition reaction initiated at an angular location of $315^{\circ}$, we can estimate the propagation speed of the reaction along the outer wall of the copper assembly in Shot 6 . The reaction speed estimated from the accelerometer data is presented in Table 9.2. 
Table 9.2. Shot 6 Outer-Liner Reaction Speed from Accelerometer Data

\begin{tabular}{|c|c|c|c|}
\hline \multirow{2}{*}{ Accelerometer } & Arc Length & \multicolumn{2}{|c|}{ Reaction Speed (cm/ $\mathbf{\mu s )}$} \\
\cline { 3 - 4 } & from Ignition Location $\mathbf{( c m )}$ & Test & Code \\
\hline 1 & 26.5 & 0.32 & 0.28 \\
\hline 2 & 16.9 & 0.31 & 0.22 \\
\hline 3 & 19.3 & 0.24 & 0.22 \\
\hline
\end{tabular}

The average reaction speed along the copper outer wall, estimated from the accelerometer test data, is $0.29 \mathrm{~cm} / \mu \mathrm{s}$; the corresponding average reaction speed estimated from the simulations is $0.24 \mathrm{~cm} / \mu \mathrm{s}$, which represents an underestimation of approximately $20 \%$. For comparison purposes, note that the sound speed for copper is $0.38 \mathrm{~cm} / \mu \mathrm{s}$.

\subsection{Resistance Wire Data}

The RW data provide information about the expansion behavior of the outer liner of the LSAC assembly. The comparison between the true strain ${ }^{1}$ calculated from the RW data in Shot 6 and the simulations for the degraded PBX-9501 is shown in Fig. 9.2.

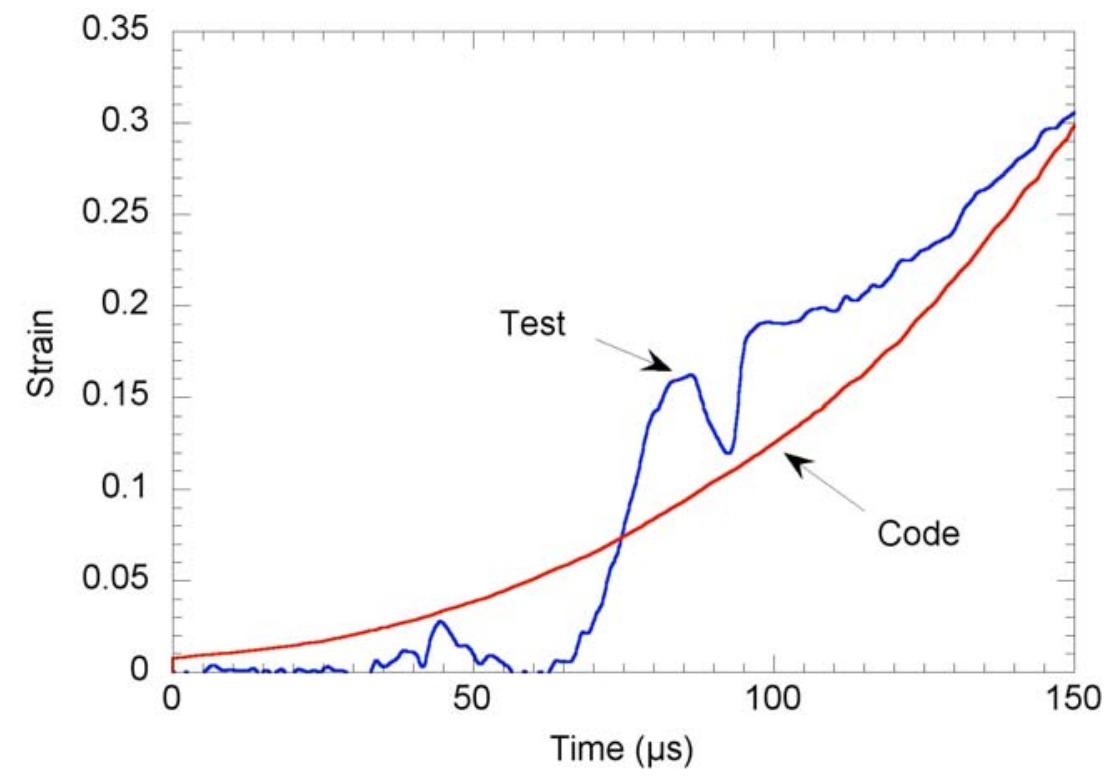

Fig. 9.2. Comparison between the RW data from Shot 6 and PAGOSA simulations for degraded PBX-9501.

The results in Fig. 9.2 correspond to the average of the values for the Lagrangian tracer particles that were located every $30^{\circ}$ around the perimeter of the middle region of the assembly, assuming that the casing remained intact. The strain recorded from the RW in Fig. 9.2 was estimated from RW 2, located around the

\footnotetext{
${ }^{1}$ The true strain $\varepsilon$ is defined as $\varepsilon=\ln \left(l / l_{0}\right)$.
} 
middle region of the outer liner of the assembly. The strain from the test data shows two distinct expansion rates: the first from approximately 60 to $85 \mu$ s and the second from about 100 to $150 \mu$ s. In contrast, the average simulated strain shows a uniform expansion rate. In general, the code calculations underestimate the observed expansion rate. The simulated plastic strain in the copper casing at the midplane region at $150 \mu$ s is shown in Fig. 9.3. Notice the azimuthal nonuniformity of the plastic strain at the midplane of the outer liner. The outer liner experiences the largest plastic strain at an angular location around $315^{\circ}$ where the thermal runaway initiated in Shot 6.

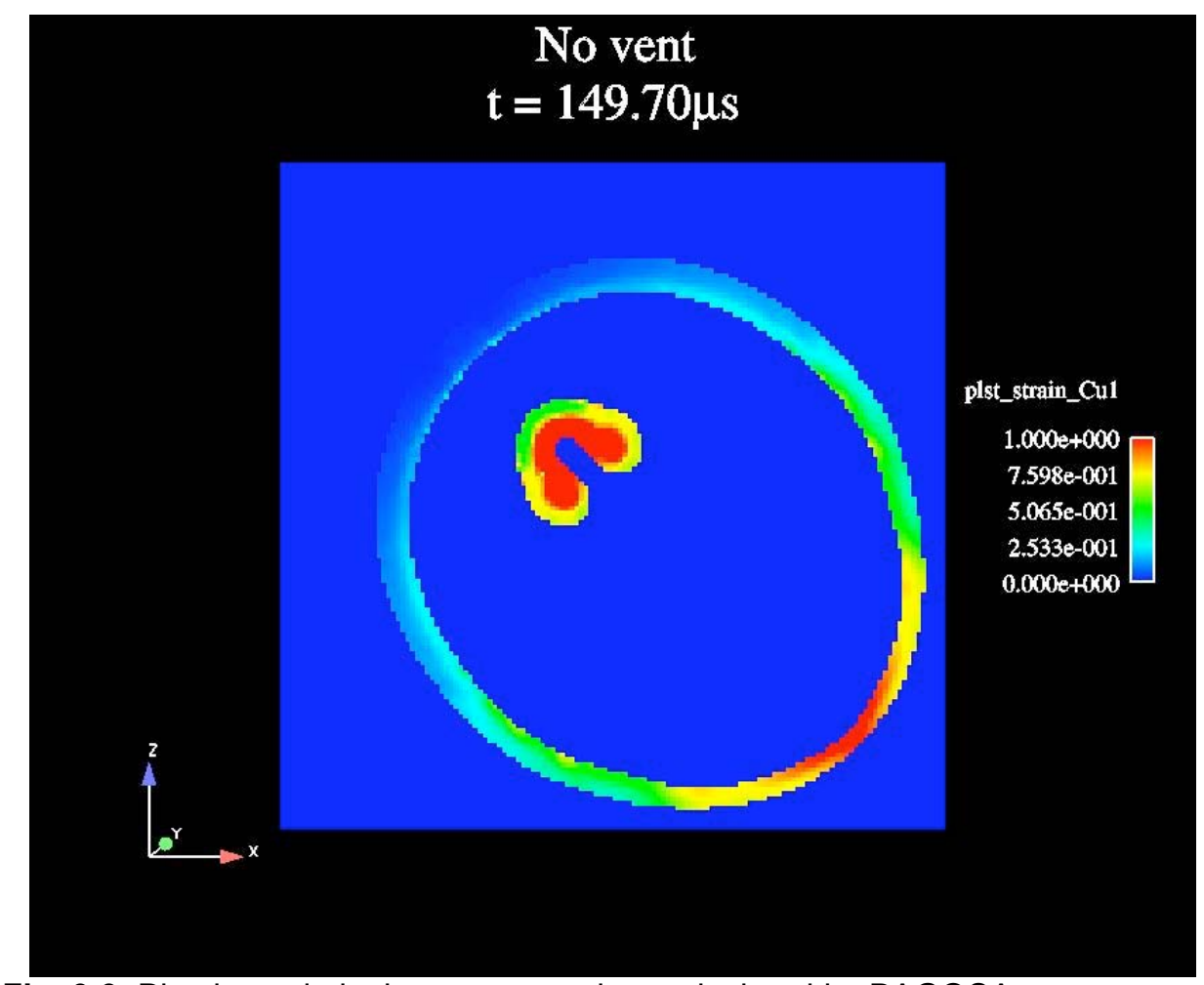

Fig. 9.3. Plastic strain in the copper casing, calculated by PAGOSA.

In contrast, the region around $135^{\circ}$, which is opposite of the location where we believe the thermal runaway initiated, practically experiences almost no plastic strain at $150 \mu$ s when the inner liner collapses.

In Shot 6, notice the sudden drop in the strain measured by the RW between approximately 80 and $90 \mu \mathrm{s}$. This change in strain, and the corresponding inner-liner deceleration that can be seen by comparing the high-speed photos at 70 and $100 \mu \mathrm{s}$, may be explained as follows: During cookoff conditions, the energy required to drive the movement of the assembly casing is caused by the decomposition of the energetic material and further combustion of the decomposition gases. These chemical reactions do not correspond to detonation conditions and progress at a much slower rate. At approximately $85 \mu \mathrm{s}$, we believe that the end caps in Shot 6 separated from the main body of the LSAC assembly, causing the release of decomposition gases and the 
subsequent release of energy otherwise available for casing deformation. This reduction in the available energy prompted the inner-liner deceleration and the reduction in the strain observed in this shot. The models we used in our simulation of Shot 6 cannot account for the reduction of the available energy when the end caps separated and therefore cannot simulate the behavior observed in Shot 6 after $85 \mu$ s.

\subsection{Velocity Interferometer System for Any Reflector Data}

The VISAR data collected during Shot 6 provide information about the end-cap wall velocity. The comparison between the end-cap velocity measured by the VISAR in Shot 6 and the velocity calculations made with PAGOSA for degraded PBX-9501 is shown in Fig. 9.4.

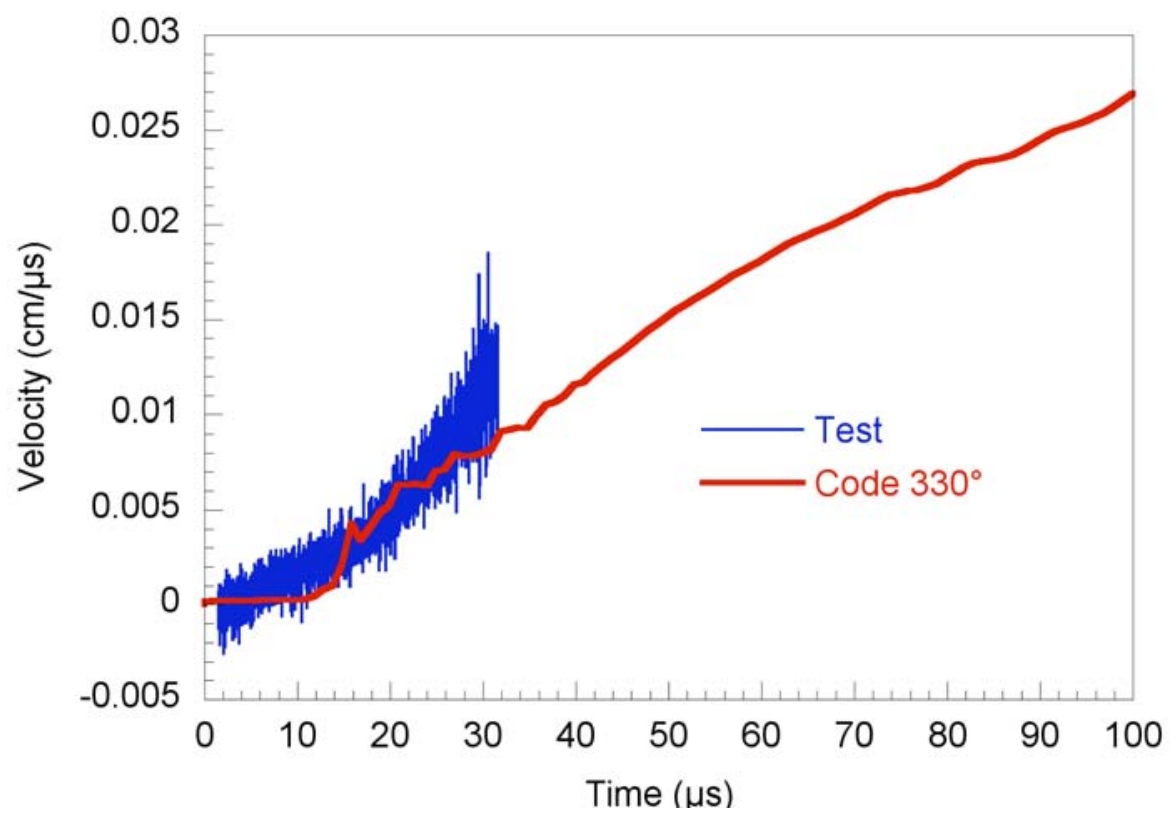

Fig. 9.4. Comparison between the VISAR data from Shot 6 and the PAGOSA calculations for degraded PBX-9501.

The VISAR used in this test was pointing toward an angular location around $330^{\circ}$. The simulation results shown in Fig. 9.4 correspond to a Lagrangian tracer particle on the end cap at an angular location of $330^{\circ}$, that is, $15^{\circ}$ from the location where the reaction started in Shot $6\left(315^{\circ}\right)$. Our simulations for the deflagration of PBX-9501 with a characteristic velocity of $1 \mathrm{~km} / \mathrm{s}$ adequately represent the observed end-cap velocity measured by the VISAR during Shot 6 . At $30 \mu$ s into the second phase of the experiment, both the code calculations and the VISAR data indicate that the end-cap velocity at an angular location of $330^{\circ}$ is approximately $10^{-2} \mathrm{~cm} / \mu \mathrm{s}(0.1 \mathrm{~km} / \mathrm{s})$. However, our simulations indicate that the end-cap velocity would be $0.2 \mathrm{~km} / \mathrm{s}$ at $70 \mu$ s and would exceed $0.3 \mathrm{~km} / \mathrm{s}$ in about $120 \mu \mathrm{s}$. The end-cap velocity at $70 \mu \mathrm{s}$ is expected to be lower than the inner-liner FMP velocity obtained from the high-speed photographs at this time because ignition appeared to have occurred in a region close to the midplane of the 
assembly in Shot 6 and the mass of an end cap is approximately 4 times the mass of the inner liner. 


\section{Interpretation of the Irregularities in the Field of View in the Collapsing Liner Images of Shot 6}

In Chapter 6, we discussed the implausibility of having a symmetric inner-liner collapse in Shot 6. In Chapter 3, we discussed that the early stages (the first $30 \mu \mathrm{s}$ ) of Shots 3 and 6 were very similar in topology and timing and thus it was possible that Shot 3 could have been qualitatively similar to Shot 6 at later times. In Shot 6, for example, some researchers have argued that the obscuring matter seen in all of the images photographed after $80 \mu \mathrm{s}$ is metal (Fig. 10.1), propelled toward the center of the assembly by the collision of the shock waves opposite from where the initiation started. However, the time scales associated with the observed collapse in the photographs indicate that the reaction advanced at subsonic speed, on the order of $1 \mathrm{~km} / \mathrm{s}$. Therefore, in Shot 6 , thermal runaway started as burning or deflagration and not as a detonation.

Researchers have also speculated that metal fragments could have been shed from the inner liner as a result of ductile spalling fracture. Therefore, knowing the tensile stress and density histories to which the inner liner is being subjected is important in determining whether or not metal fragments would be shed during implosion of the liner. The pressure level would also be a determining factor in establishing whether the liner could become unstable and, in turn, lead to the plastic flow of metal. The possibility that spall fracture and Rayleigh-Taylor instability might have occurred during the LSAC assembly tests (particularly in Shot 6) is discussed in this chapter.

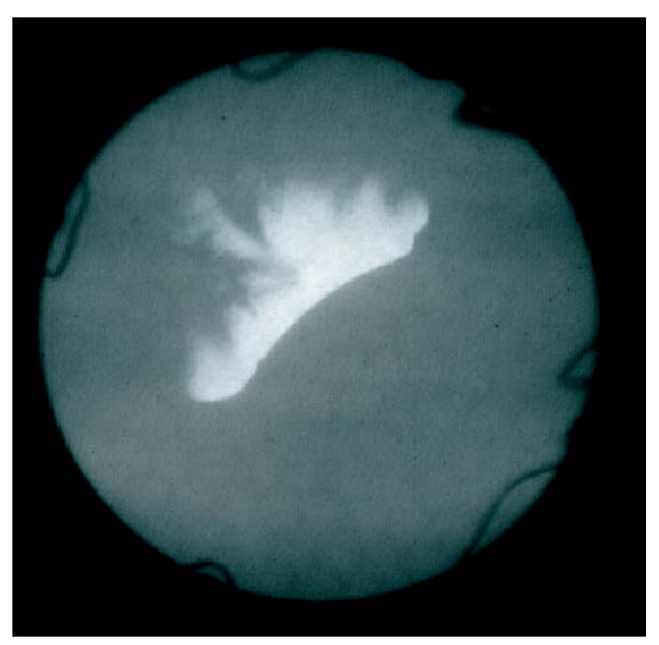

Fig. 10.1. Shot 6 framing camera image at $80 \mu \mathrm{s}$. (Photo provided by $D X-2$.

\subsection{Spalling Fracture}

Spalling occurs within a metal when microscopic voids nucleate under tension, grow, and coalesce, leading to fracture. The formation of voids within the damaged metal is referred to as porosity. For this phenomenon to occur, a threshold tensile stress must be 
exceeded and last about $1 \mu \mathrm{s}$. For cold-drawn copper, this threshold is $1 \mathrm{GPa}$, and for annealed copper, it is $0.5 \mathrm{GPa}$ (Wu et al. 2003). Some investigators believe that spallation of the inner liner could be a source of metal fragments ejected into the field of view shown in the high-speed framing camera images of the LSAC assembly shots.

We performed independent numerical simulations with PAGOSA and MESA-2D (Holian et al. 1989) to determine whether this spall-fracture claim had merit. We used PAGOSA to compute the mean stress around the inner liner in a configuration whose pin curves (see Chapter 8) were consistent with the data for Shot 6. With MESA-2D, we computed the porosity distribution within the inner liner for pristine and cooked PBX9501 (reaction front velocity of $1 \mathrm{~km} / \mathrm{s}$ and $100 \%$ of $E_{P B X-9501}$ ).

\subsubsection{PAGOSA Simulations}

In cooked $\mathrm{HE}$, the pressure in the liner remains low at every instant before the complete collapse, as shown in Fig. 10.2, where the pressure is plotted as a function of time for a tracer particle located at $r=4.13 \mathrm{~cm}, \theta=90^{\circ}$, and $z=0 \mathrm{~cm}$. Figure 10.2 shows that the collapse of the inner liner is characterized by the $68 \mathrm{GPa}$ pressure spike that occurs at $155 \mu \mathrm{s}$. Note that this is the simulation time and does not correspond to the time scale in the high-speed photographs taken of Shot 6 . Before the collapse of the inner liner, the maximum tension achieved has a magnitude of $0.25 \mathrm{GPa}$ and occurs at $148.4 \mu \mathrm{s}$. Our simulations show that no particle within the inner liner was subjected to a tension comparable to the spall strength of copper (cold-drawn or annealed) before $150 \mu$ s. Therefore, the simulated stresses produced by the explosion of a thermally damaged HE that approximates the cooked PBX-9501 for Shot 6 do not reach the minimum critical levels to effect ductile spalling fracture. Because of these computations, we believe that no spalling could have occurred during this shot. A more plausible explanation for the sharp features observed in Figure 10.1 is that they were formed as a result of the explosive dispersal of gases that came into the camera's field of view when the end cap failed due to the pressure generated by the reacting HE.

In the pristine case shown in Fig. 10.3 (at the same material particle location), a completely different time dependence is observed for the pressure. There is an early peak pressure of $27 \mathrm{GPa}$ at about $5 \mu \mathrm{s}$, which is the time it took for the shock front to travel from the initiation point to the middle of the inner liner. This peak is followed, $2 \mu \mathrm{s}$ later, by a tension of $6 \mathrm{GPa}$, which eventually levels off after $20 \mu$ s to a pressure of $3 \mathrm{GPa}$ before monotonically increasing to the peak pressure of $109 \mathrm{GPa}$. Thus, the pressure exerted on the liner remains no greater than $30 \mathrm{GPa}$ before the opposite ends of the inner liner collide. Our simulations show that spalling fracture is physically possible at or near the region around $r=4.13 \mathrm{~cm}, \theta=90^{\circ}$, and $z=0 \mathrm{~cm}$ because the computed tensile stress surpasses the spall strength. As shown in Fig. 10.3, spalling could have occurred in this location at three different times: $6.8 \mu \mathrm{s}, 10 \mu \mathrm{s}$, and $13.6 \mu \mathrm{s}$. 


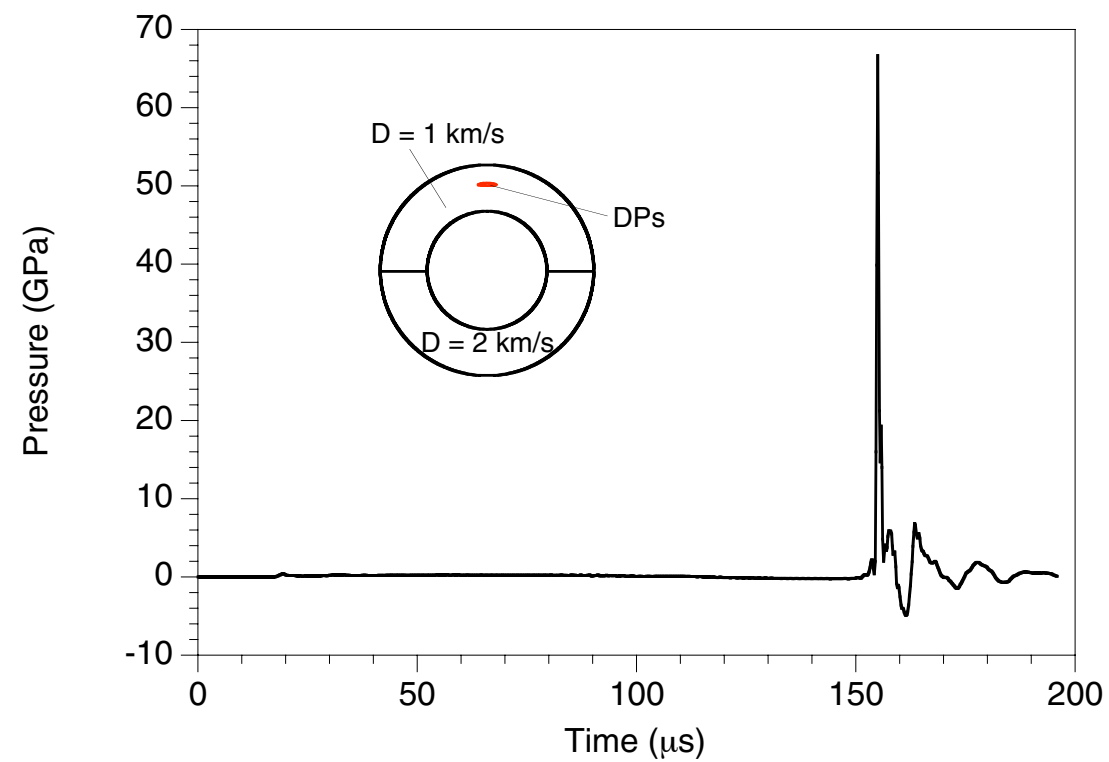

Fig. 10.2. Pressure as a function of time for a tracer particle located at $r=4.13 \mathrm{~cm}, \theta=90^{\circ}$, and $z=0$. One-half of the $\mathrm{HE}$ has $\mathrm{D}=1 \mathrm{~km} / \mathrm{s}$, and the other half has $2 \mathrm{~km} / \mathrm{s}$; both halves have $100 \%$ of $E_{P B X-9501}$ (initiation points are on the locus of a small ellipsoid close to the outer liner).

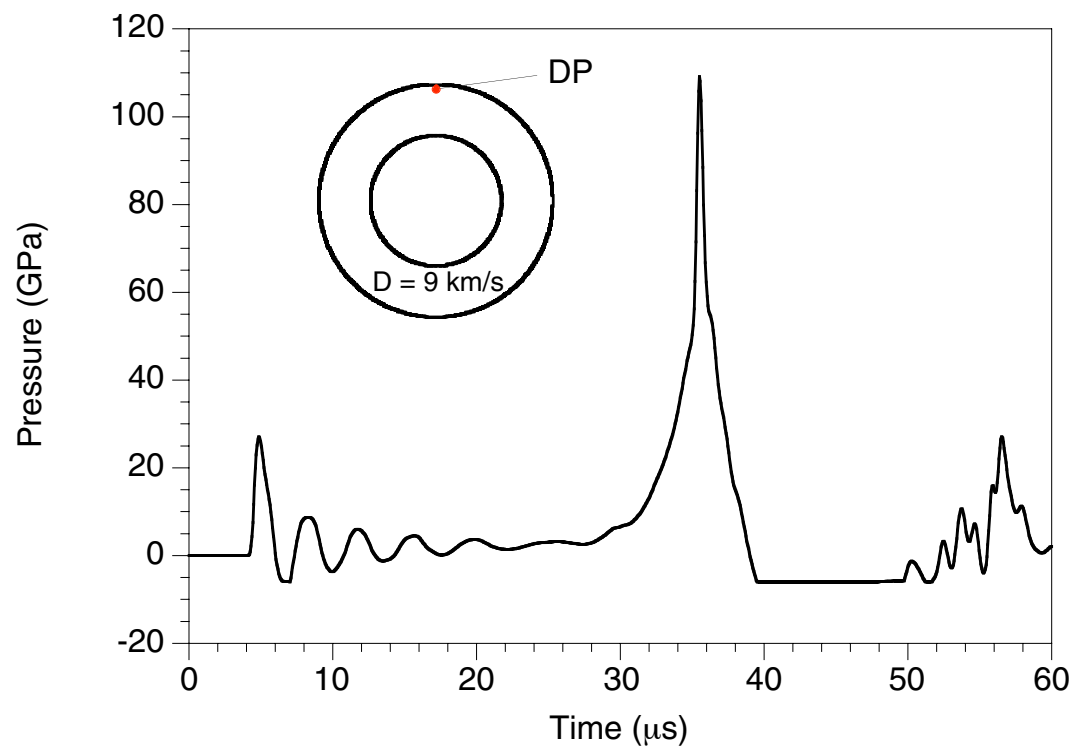

Fig. 10.3. Pressure as a function of time for a tracer particle located at $r=4.13 \mathrm{~cm}, \theta=90^{\circ}, z=0$. The entire HE is pristine PBX-9501 (D $\left.=9 \mathrm{~km} / \mathrm{s}\right)$, and the detonation point is located near the outer liner.

\subsubsection{MESA-2D Simulations}

Two MESA-2D (Holian et al. 1989) Eulerian simulations were also performed to investigate the possibility of spall damage to the inner liner. The simulations assumed a plane-strain approximation zoning that was adequate to resolve the shock and release 
waves traveling in the copper liner, and they incorporated the mechanical threshold stress strength model (Follansbee and Kochs 1988) and the Johnson spall (JS) model (Johnson 1981; Johnson and Addessio 1988). The basic JS model parameter set for copper was used. Although some of the model's parameters are empirically based and have been adjusted to reproduce the flyer-plate impact (uniaxial strain) experimental data for copper, it should be noted that the wave structure for metal loading and/or unloading by explosives (Taylor wave) and for metal loading by impact (as in the flyer-plate experiments) are different and that this model has been validated for metal loading by impact.

The first simulation assumed the pristine parameters for the PBX-9501 detonation velocity and JWL EOS, whereas the second simulation utilized the thermally damaged PBX-9501 reaction rate and the JWL EOS that reproduced the early-time motion of the inner liner observed in LSAC Shot 6. In both simulations, the outer liner was included, and the explosive was assumed to initiate at a single point (close to the outer liner, as observed in LSAC Shot 6). Figures 10.4 through 10.6 show the time-sequenced evolution of the pressure response and damage (porosity) accumulated in the inner liner as the detonation wave/reaction front interacts with the copper inner liner.
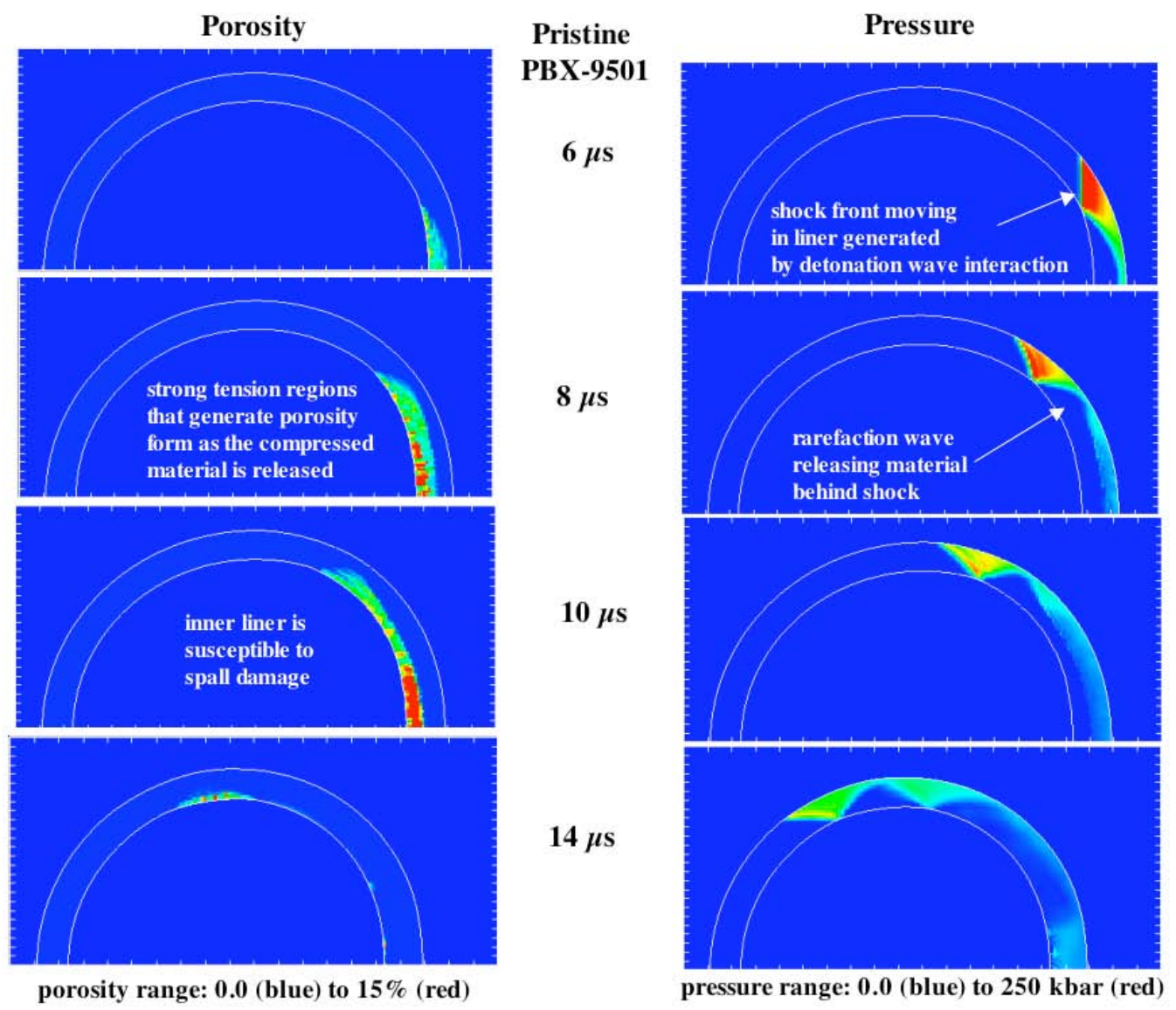

Fig. 10.4. Pressure and porosity profiles, as a function of time, for pristine PBX9501 between 6 and $14 \mu \mathrm{s}$. 
In the pristine simulation, shown in Figs. 10.4 and 10.5, significant porosity (15\%) is rapidly generated on the inner surface of the liner following the release of the shock wave. In this simulation, spall damage is likely and is concentrated around the region where the detonation wave interacts with the liner and on the opposite side where the two detonation waves collide. No analyses were performed to determine if the spall fragments would be ejected into the annulus.
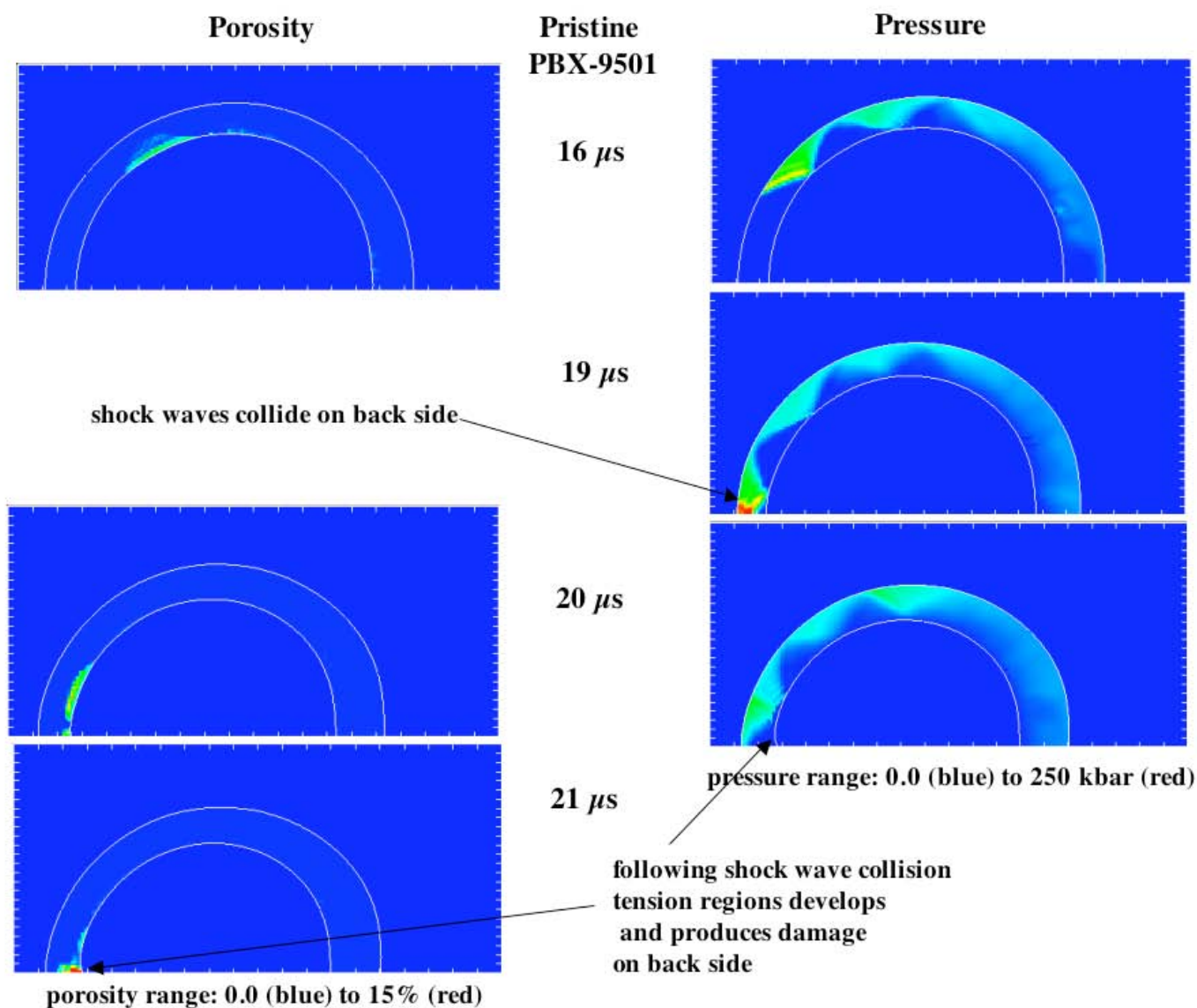

Fig. 10.5. Pressure and porosity profiles, as a function of time, for pristine PBX9501 between 16 and $21 \mu$ s.

In the thermally damaged PBX-9501 simulation, shown in Fig. 10.6, the relatively weak explosive drive (energy-release rate with respect to the pristine case) by deflagration does not produce significant stress gradients or generate enough porosity to cause spall damage in the inner liner at any azimuthal position (the code simulation predicted a maximum porosity of $\sim 1 \%$ ). 


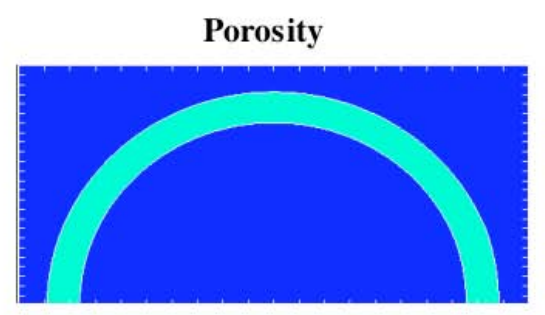

"Degraded"

PBX-9501

$40 \mu \mathrm{s}$
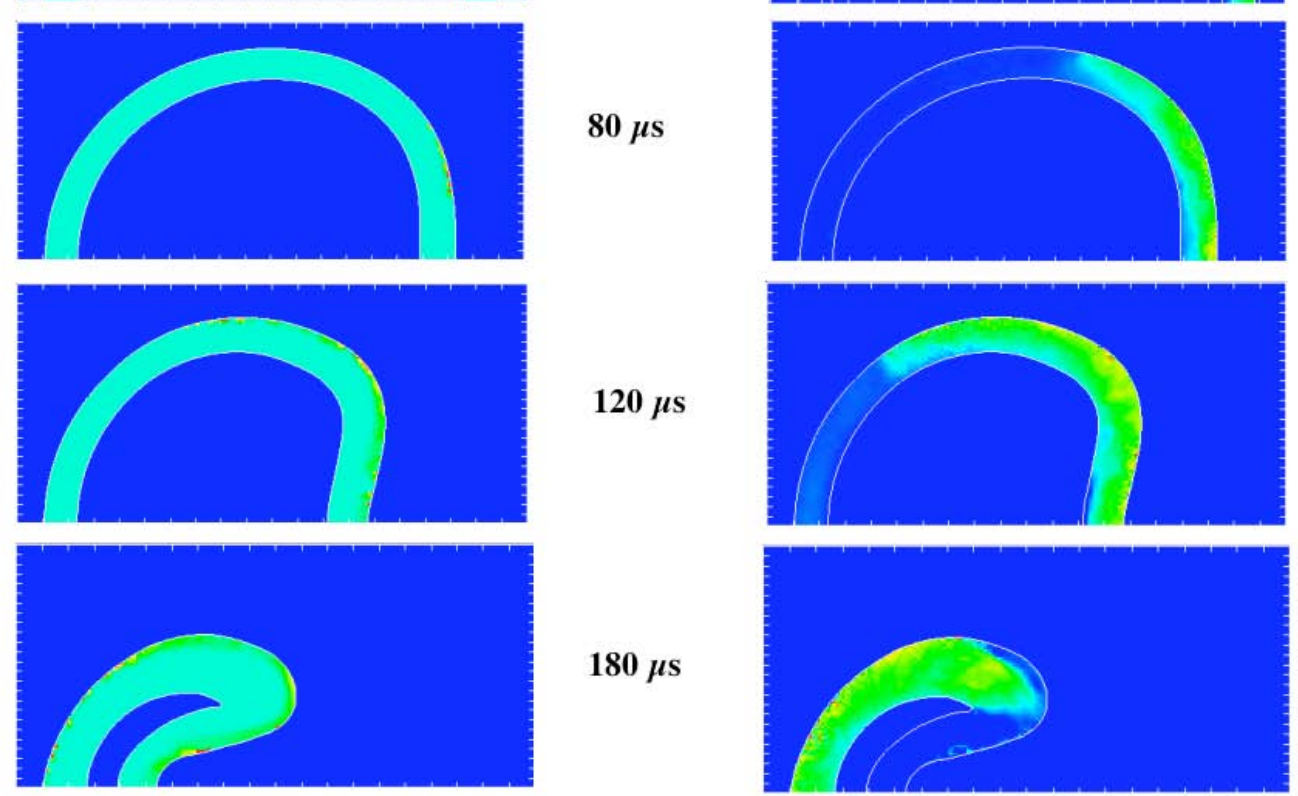

porosity range: $0.3 \%$ (cyan) to $1.0 \%$ (red)

pressure range: 0 (blue) to 1 kbar (red)

Fig. 10.6. Pressure and porosity profiles, as a function of time, for thermally damaged or degraded PBX-9501 between 40 and $180 \mu \mathrm{s}$.

The simulation of the thermally damaged HE suggests that spall fragments would not be produced, and therefore they would not be the material ejected into the center of the assembly, as seen in the camera's field of view during the LSAC experiments.

\subsection{Rayleigh-Taylor Instability}

Another argument proposed by the experimentalists to explain the supposed metal seen in the field of view in Fig. 10.1 was that the inner liner underwent a hydrodynamic instability. When a metal plate is subjected to a sufficiently high acceleration or pressure gradient across its thickness, it may become unstable (Bakhrakh et al. 1997; Plohr and Sharp 1998). This phenomenon is similar to the well-known Rayleigh-Taylor instability in fluid systems (Chandrasekhar 1961). Although the nature of the instability is different in solids than in fluids (Swegle and Robinson 1989), the term "Rayleigh-Taylor" is often used to refer to unstable interfaces in either the liquid or solid state. Bakhrakh et al. (1997) derived the dispersion relation for an elastic semispace and also for a thin elastic layer. In addition, they developed an approximate theory for incompressible elastoplastic plates. Plohr and Sharp (1998) studied the stability of a linearly elastic solid plate by casting the governing equations as an initial boundary value problem. Both of these authors focused their analyses on the cutoff wavelength, which they referred to as the 
"critical" wavelength. However, disturbances whose wavelengths correspond to those at the cutoff are marginally stable, that is, their growth rate is zero. In stability analyses, one seeks the wavelength $\lambda$ of disturbances for which the growth rate is maximum, i.e., the most unstable modes (Terrones 2005). Here, in order to estimate the necessary conditions that will lead to a Rayleigh-Taylor type of instability of the inner liner in Shot 6, we calculate the most unstable disturbance because it is the one that will grow the fastest. This is the disturbance that will be "naturally" amplified in the absence of preexisting imperfections in the metal layer (Terrones 2005). For an elastic metal plate of arbitrary thickness $h$, Plohr and Sharp (1998) derived the following relationship between the wave number of the disturbance $k(=2 \pi / \lambda)$ and its growth rate $\sigma$ :

$$
\left(\frac{p k}{G h}\right)^{2}=\frac{\left(q^{2}+k^{2}\right)^{4}+16 q^{2} k^{6}-8 q k^{3}\left(q^{2}+k^{2}\right)^{2}(\operatorname{coth} k h \operatorname{coth} q h-\operatorname{csch} k h \operatorname{csch} q h)}{\left(q^{2}-k^{2}\right)^{2}},
$$

where

$$
q=\sqrt{k^{2}+\frac{\rho \sigma^{2}}{G}}
$$

and $p$ is the applied pressure at one end of the metal layer, $G$ is the shear modulus, and $\rho$ is the density.

Since the fastest growing mode is a maximum, it satisfies $\partial \sigma(p, G, h, k) / \partial k=0$ (which can be derived from Eq. (10.1) by implicit differentiation). From this and Eq. (10.1), a system of two transcendental equations must be solved for the maximum growth rate and the most unstable wave number. Therefore, for a given applied pressure, the wavelength of the fastest growing mode can be numerically calculated by solving the above system of equations. Figure 10.7 shows the dimensionless most-unstable $\left(\lambda_{m} / h\right)$ and cutoff $\left(\lambda_{c} / h\right)$ wavelengths as functions of the dimensionless pressure $(p / G)$. These curves can be approximated by

$$
\frac{\lambda_{m}}{h} \approx 13.3\left(\frac{G}{p}\right)^{0.64} \text { and } \frac{\lambda_{c}}{h} \approx 6.6\left(\frac{G}{p}\right)^{0.51} \text {. }
$$




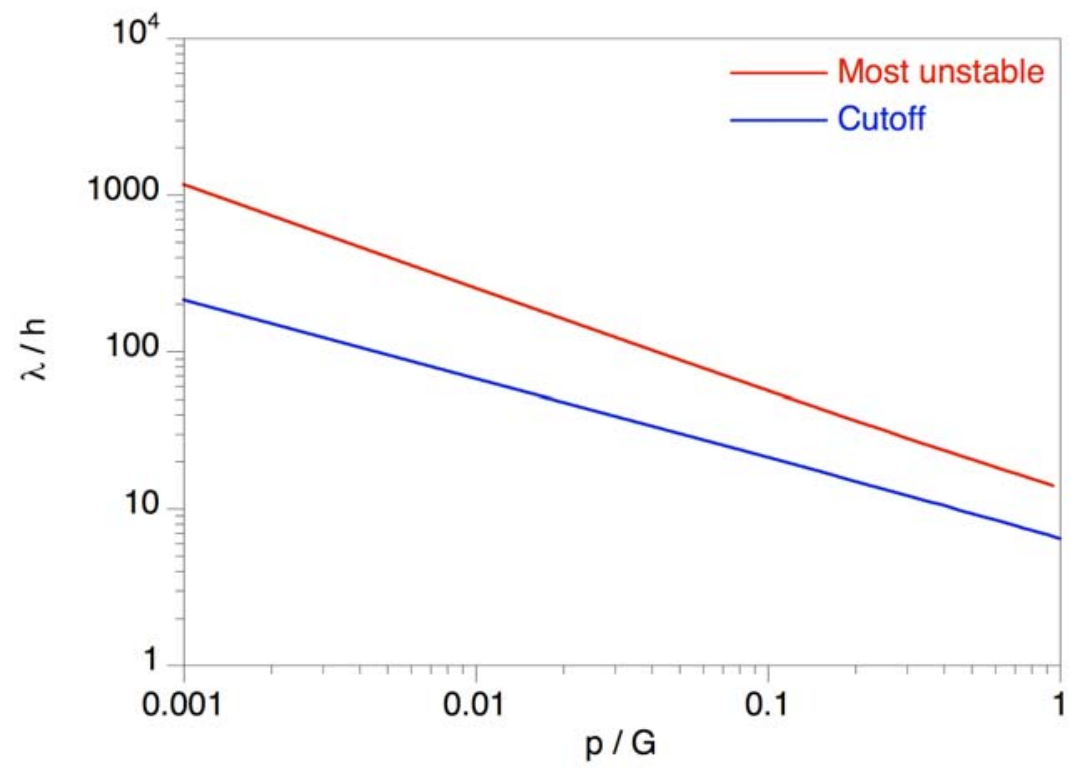

Fig. 10.7. Dimensionless most-unstable and cutoff wavelengths, as functions of the normalized pressure.

From the computed pressure shown in Fig. 10.2, we can see that the maximum pressure to which the metal layer was subjected before collapse was $0.5 \mathrm{GPa}$. For copper, the initial shear modulus is $47.7 \mathrm{GPa}$ (Steinberg 1996); therefore, $p / G \approx 0.01$, and from Eq. (10.2), $\lambda / h \approx 250$. Under these conditions and for a layer thickness of $0.635 \mathrm{~cm}$ (inner-liner thickness), the length scale required to destabilize the system would have to be $1.56 \mathrm{~m}$. Taking into account the decrease in $G$ due to temperature, say at $200^{\circ} \mathrm{C}$, would decrease only the most-unstable wavelength to about $1.5 \mathrm{~m}$. Using the wavelength associated with the marginally stable disturbance (the cutoff wavelength) yields a length scale of $0.43 \mathrm{~m}$, which at $200^{\circ} \mathrm{C}$ reduces to $0.41 \mathrm{~m}$. Clearly, these length scales do not exist for the LSAC assembly of any shot tested. Therefore, the inner liner in Shot 6 cannot become unstable via the Rayleigh-Taylor instability. Similarly, it can be shown that even for the pressure levels attained with pristine PBX-9501, the Rayleigh-Taylor instability is unlikely. In this case, the length scale required to destabilize the system would have to be $11 \mathrm{~cm}$.

Experimentally, to artificially induce a predetermined Rayleigh-Taylor mode to grow in a plate, it is necessary to machine a grooved pattern with a depth $\Delta h$ and wavelength $\lambda(=2 \pi / k)$ on the metal. For a sinusoidal pattern machined on a plate, Lebedev et al. (1996) derived the following equation for the amplitude of the disturbance in terms of the mechanical properties of the metal:

$$
\Delta h=\frac{2 h Y}{p}\left(1-a e^{-k h / \sqrt{3}}\right)\left[\left(1-e^{-k h / \sqrt{3}}\right)^{2}-\left(\frac{p}{2 G k h}\right)\right],
$$

where $Y$ is the yield strength and $a$ is a dimensionless constant $(=0.856)$. 
If we choose a $3-\mathrm{cm}$ wavelength for a copper plate that is $0.635 \mathrm{~cm}$ thick, where $Y=0.64 \mathrm{GPa}$, the required groove depth for Rayleigh-Taylor instability is $0.3 \mathrm{~cm}$. Since the inner and outer liners of every shot had nominally smooth surfaces, it is improbable that imperfections as large as half of the thickness of the liner would be present at the time of the tests. Therefore, in our opinion, no Rayleigh-Taylor instability could have occurred in any of the shots.

Furthermore, since the HE is adjacent to the outer perimeter of the inner liner, if a Rayleigh-Taylor instability were to occur, it would first be visible at the outer diameter. Once the instability was initiated, a pattern of bubbles and spikes would appear. The spikes would be directed radially outward toward the gases produced by the HE, which are accelerating the liner, but the bubbles would grow radially inward. Thus, the bubbles would be directed radially inward toward in the field of view. Instead, what is observed in Fig. 10.1 is a pattern not of bubbles and spikes but of "fuzzy" wedgelike streaks. 


\section{Conclusions}

We analyzed the available data from the large-scale annular cookoff (LSAC) assembly series of experiments to understand the implications that cookoff of PBX-9501 could have on safety assessments. In addition, we examined recent data on the permeability of cooked PBX-9501 before ignition because of its potential relevance to post-ignition behavior. The conclusions of our analyses supported by analytical and numerical tools are listed below.

From the set of unambiguous high-speed photographs, we found that thermal initiation was localized in all cases. At the beginning of the inner liner collapse, a welldefined clear-cut almost flat interface is observed. For instance, the wall deformation observed from the high-speed photographs for Shot 3 at $25 \mu$ s and Shot 6 at $30 \mu$ s are very similar. Both shots exhibit localized displacement with a small curvature interface where the fastest moving point in each case has similar speeds. High-speed photographs show two localized regions in Shot 4, which are almost diametrically opposed to each other and that appear to have ignited within a couple of microseconds. A second hot spot was not detected experimentally because two thermocouples at the same angular location failed, which coincidentally, were located near the second initiation region. Localized initiation precluded perfectly symmetric collapse, i.e., uniform diminution of the inner liner radius.

Based on data from recent permeability experiments, we believe that convective transport of liquid binder at $185^{\circ} \mathrm{C}$ has been recorded but not recognized as such. Models based on treating PBX-9501 as a single-phase gas-saturated homogeneous porous medium (governed by Darcy's law) during the pre-ignition stage, when temperatures are around $180^{\circ} \mathrm{C}$, are not supported by the experimental data supplied to the authors. Numerical simulations of pre-ignition based on conductive heat transfer with appropriately tuned kinetic parameters are in excellent agreement with experiments. Therefore, invoking other uncertain physical mechanisms for pre-ignition modeling is unwarranted.

Our metric for symmetry shows that no shot, for which there is a set of unambiguous high-speed photographs, exhibited perfectly symmetric collapse.

Shot 6 was the only experiment with enough information to develop an approximate equation of state for the gaseous products of deflagration that can be used in a hydrodynamics code to simulate post-ignition due to cookoff during the first $100 \mu \mathrm{s}$. Our analysis shows that the inner-liner collapse behavior in Shot 6 is consistent with a peak pressure of approximately $8 \mathrm{kbar}$, a propagation speed of the deflagration front of $1 \mathrm{~km} / \mathrm{s}$, and a point initiation close to the outer liner. The peak pressure is much higher than the yield stress at the Hugoniot elastic limit and also higher than the ultimate strength for copper. Therefore, this pressure was sufficiently high to destroy the copper assembly without having a detonation.

The stress levels on the inner liner of the assembly produced by a peak pressure of 8 kbar are very low to generate spall fracture or a hydrodynamic instability. Therefore, the obscuring matter with shade gradients that appears on the high-speed photographs is most likely gas resulting from confinement failure at the end caps. 


\section{Acknowledgments}

The authors gratefully acknowledge insightful discussions with Langdon S. Bennett regarding the use of the Jones-Wilkins-Lee equation of state to approximate a deflagration and for his careful review of the manuscript. We also thank Blaine Asay, Peter Dickson, Gary Parker, Laura Smilowitz, and Bryan Henson for providing their data on the Big Shot series and the permeability experiments. Thanks to Larry Luck and David Zerkle of Los Alamos National Laboratory Group D-5, who provided us with several computational results from their pre-ignition model. Finally, we greatly appreciate our interactions with Brian H. Aubert, Temo Aviles-Ramos, and Renida R. Carter. 


\section{References}

Asay, B. W., P. Dickson, B. Henson, L. Smilowitz, L. Tellier, N. Glassmaker, and M. Greenfield, "Large Scale Annular Cookoff Experiment (LSAC)," Joint Army-NavyNASA-Air Force (JANNAF) Propulsion-Systems Hazards Meeting, Cocoa Beach, Florida, October 18-20, 1999, Los Alamos National Laboratory report LA-UR-99 5203 (1999).

Asay, B. W., T. Schaefer, P. Dickson, B. Henson, and L. Smilowitz, "Measurement of Gas Permeability in Thermally Damaged PBX-9501," Los Alamos National Laboratory report LA-UR-02-1755 (2002).

Asay, B. W., P. Dickson, B. Henson, D. Oschwald, G. Parker, L. Perry, J. Romero, and L. Smilowitz. CD of the Data for the LSAC Assembly Tests (which is an IGOR PRO packed experiment file), Los Alamos National Laboratory report LA-UR-03-2549 (2003).

Asay, B. W., G. Parker, P. Dickson, B. Henson, and L. Smilowitz, "Dynamic Measurement of the Permeability of an Explosive Undergoing Thermal Damage," $J$. Energ. Mater. 22 (1), 25-39 (2004).

Aviles-Ramos, C., "Three-Dimensional Finite Element Simulations of the LANL Large Scale Annular Cook-Off Experiments Designated as Shot 5, Shot 6, Shot 7, and Shot 8," Los Alamos National Laboratory report LA-UR-04-2870 (2004).

Bakhrakh, S. M., O. B. Drennov, N. P. Kovalev, A. I. Lebedev, E. E. Meshkov, A. L. Mikhailov, N. V. Nevmerzhitsky, P. N. Nizovtsev, V. A. Rayevsky, G. P. Simonov, V. P. Solovyev, and I. G. Zhidov, "Hydrodynamic Instability in Strong Media," Lawrence Livermore National Laboratory report UCRL-CR-126710 (1997).

Behrens, R., "Thermal Decomposition of HMX: Morphological and Chemical Changes Induced at Slow Decomposition Rates," $12^{\text {th }}$ International Detonation Symposium, San Diego, California, August 11-16, 2002.

Behrens, R., "Thermal Decomposition Studies of Energetic Materials: HMX, ONDNTA, TNAZ, 24DN1, ANTA and DNBT," Sandia National Laboratories report SAND20038327P (2003).

Berghout, H. L., S. F. Son, C. B. Skidmore, D .J. Idar, and B. W. Asay, "Combustion of Damaged PBX 9501 Explosive,” Thermochim. Acta 384, 261-277 (2002).

Bird, R. B., W. E. Stewart, E. N. Lightfoot, Transport Phenomena, (Wiley, New York, 1960).

Brill, T. B. and R. J. Karpowicz, "Solid-Phase Transition Kinetics. The Role of Intermolecular Forces in the Condensed-Phase Decomposition of Octahydro-1,3,5,7Tetranitro-1,3,5,7-Tetrazocine," J. Phys. Chem. 86, 4260-4265 (1982).

Cady, C. M., W. R. Blumenthal, G. T. Gray III, and D. J. Idar, "Mechanical Properties of Plastic-Bonded Explosive Binder Materials as a Function of Train-Rate and Temperature," Los Alamos National Laboratory report LA-UR-00-3132 (2000). 
Chandrasekhar, S., Hydrodynamic and Hydromagnetic Stability, (Oxford University Press, 1961).

Cooper, P. W., Explosive Engineering (Wiley-VCH, New York, 1996).

Dickson, P. M., B. W. Asay, B. F. Henson, C. S. Fugard, and J. Wong, "Measurement of Phase Change and Thermal Decomposition Kinetics Using the Los Alamos Radial Cookoff Test," Los Alamos National Laboratory report LA-UR-99-3272 (1999).

Dickson, P. M., B. W. Asay, B. F. Henson, C. S. Fugard, and J. Wong, "Measurement of Phase Change and Thermal Decomposition Kinetics during Cookoff of PBX-9501," in Shock Compression of Condensed Matter, M. D. Furnish, L. C. Chabildas, and R. S. Hixon, Eds. (American Institute of Physics, Melville, New York, 2000) pp. 837-840.

Estane Material Safety Data Sheet, 2001.

Estane Technical Data Sheet, 2001.

Explosives Reference Guide, October 2004, Lawrence Livermore National Laboratory document UCRL-WEB-207690.

Fetter, C. W. Contaminant Hydrogeology (Prentice Hall, New Jersey, 1999).

Fickett, W. and W. C. Davis, Detonation Theory and Experiment, (Dover Publications, Mineola, New York, 2000).

Follansbee, P. S. and U. F. Kochs, "A Constitutive Description of the Deformation of Copper Based on the Use of the Mechanical Threshold Stress as an Internal State Variable," Acta Metall. 36 (1), 81-93 (1988).

Grady, D. E. and M. E. Kipp, "Mechanisms of Dynamic Fragmentation-Factors Governing Fragment Size," Mechanics of Materials 4 (3-4), 311-320 (1985a).

Grady, D. E. and M. E. Kipp, "Geometric Statistics and Dynamic Fragmentation," J. Appl. Phys. 58 (3), 1210-1222 (1985b).

Henson, B. F., B. W. Asay, R. K. Sander, S. F. Son, J. M. Robinson, and P. M. Dickson, "Dynamic Measurement of the HMX Beta-Delta Phase Transition by Second Harmonic Generation,” Phys. Rev. Lett. 82, 1213 (1999).

Henson, B. F., L. Smilowitz, B. W. Asay, and P. M. Dickson, "The $\beta-\delta$ Phase Transition in the Energetic Nitramine Octahydro-1,3,5,7-Tetranitro-1,3,5,7-Tetrazocine: Thermodynamics," J. Chem. Phys. 117, 3780 (2002).

Holian, K. S., D. A. Mandell, T. F. Adams, F. L. Addessio, J. R. Baumgardner, and S. J. Mosso, "MESA: Computer Code for Armor/Anti-Armor Applications," Supercomputing World Conference, San Francisco, California, October 17-20, 1989.

Howe, P. M., P. M. Dickson, B. F. Henson, B. W. Asay, L. B. Smilowitz, and M. T. Greenfield, "Thermal Response of Explosives: Slow Heat," Los Alamos National Laboratory report LA-UR-002859 (2000a). 
Howe, P. M., P. M. Dickson, B. F. Henson, B. W. Asay, L. B. Smilowitz, M. T. Greenfield, J. Bennett, and J. Gerken, "Thermal Response of Explosives Systems," Los Alamos National Laboratory report LA-UR-002959 (2000b).

Johnson, J. N., "Dynamic Fracture and Spallation in Ductile Solids," J. Appl. Phys. 52 (4), $2812(1981)$.

Johnson, J. N. and F. L. Addessio, “Tensile Plasticity and Ductile Fracture,” J. Appl. Phys. 64 (12), 6699-6712 (1988).

Kaneshige, M. J., A. M. Renlund, R. G. Schmitt, and W. W. Erikson, "Cook-Off Experiments for Model Validation at Sandia National Laboratories," $12^{\text {th }}$ International Detonation Symposium, San Diego, California, August 11-16, 2002.

Kaneshige, M. J., A. M. Renlund, R. G. Schmitt, and W. W. Erikson, "Improvements in Cook-Off Models Using Real-Time in situ Measurement of Temperature and Pressure," JANNAF Propulsion-Systems Hazards Subcommittee Meeting, Colorado Springs, Colorado, December, SAND2003-4419C (2003).

Kaneshige, M.J., A. M. Renlund, and R. G. Schmitt, "Heated Triaxial Experiments on Plastic Bonded Explosives for Constitutive Model Development," Sandia National Laboratories, SAND2004-0018C (2004).

Kothe, D. B., J. R. Baumgardner, J. H. Cerutti, B. J. Daly, K. S. Holian, E. M. Kober, S. J. Mosso, J. W. Painter, R. D. Smith, and M. D. Torrey, "PAGOSA-A Massively Parallel, Multimaterial Hydrodynamics Model for 3-Dimensional High-Speed Flow and High-Rate Material Deformation," $1^{\text {st }}$ High Performance Computing Symposium, pp. 9-14, 1993.

Lebedev, A. I., P. N. Nizovtsev, V. A. Raevskii, and V. P. Solovev, "Rayleigh-Taylor Instability in Firm Substances," Physics Doklady, 41, 328-330 (1996).

Los Alamos Explosive Property Data, T. R. Gibbs and A. Popolato, Eds. (University of California Press, Berkeley, 1980).

Mader, C. L., Numerical Modeling of Explosives and Propellants, (CRC Press, Boca Raton, Florida, 1998).

Maienschein, J. L., J. F. Wardell, and J. E. Reaugh, "Thermal Explosion Violence of HMX-Based Explosives - Effect of Composition, Confinement and Phase Transition Using the Scaled Thermal Explosion Experiment," Lawrence Livermore National Laboratory UCRL-JC-138876, (2000).

Maienschein, J. L., J. F. Wardell, R. K. Weese, B. J. Cunningham, and T. D. Tran, "Understanding and Predicting the Thermal Explosion Violence of HMX-Based and RDX-Based Explosives: Experimental Measurements of Material Properties and Reaction Violence," Lawrence Livermore National Laboratory, UCRL-JC-14461, (2002).

McClelland, M. A., T. D. Tran, B. J. Cunningham, R. K. Weese, and J. L. Maienschein, "Cookoff Response of PBXN-109: Material Characterization and ALE3D Thermal Predictions," Lawrence Livermore National Laboratory Technical Report, UCRL-JC144009 (2001). 
McClelland, M. A., J. L. Maienschein, A. L. Nichols, J. F, Wardell, A. I. Atwood, and P. O. Curran, "ALE3D Model Predications and Materials Characterization for the Cookoff Response of PBXN-109," Lawrence Livermore National Laboratory report UCRL-JC-145756 (2002).

McGuire, R. R. and C. M. Tarver, "Chemical Decomposition Models for the Thermal Explosion of Confined HMX, TATB, RDX, and TNT," Seventh Symposium (International) on Detonation (Naval Surface Weapons Center, Annapolis, MD, 1981, NSWC MP 82-334, p. 56.

Nakayama, A., PC-Aided Numerical Heat Transfer and Convective Flow (CRC Press, Boca Raton, 1995).

Nichols, A. L., A. Anderson, R. Neely, and B. Wallin, "A Model for High Explosive Cookoff," Lawrence Livermore National Laboratory report UCRL-JC-144971 (2002).

Palmer, S. J. P., J. E. Field, and J. M. Huntley, "Deformation, Strengths and Strains to Failure of Polymer Bonded Explosives," Proc. R. Soc. London, Ser. A, 440, 399-419 (1993).

Parker, G. R., B. W Asay, P. Dickson, B. Henson, and L. Smilowitz, "Effect of Thermal Damage on the Permeability of PBX 9501," Los Alamos National Laboratory report LA-UR-03-4718 (2003).

Parker, G. R., P. D. Peterson, B. W. Asay, P. M. Dickson, W. L. Perry, B. F. Henson, L. Smilowitz, and M. R. Oldenborg, "Examination of Morphological Changes that Affect Gas Permeation through Thermally Damaged Explosives," Propellants, Explosives, Pyrotechnics 29, 274-281 (2004).

Plohr, B. J. and D. H. Sharp, "Instability of Accelerated Elastic Metal Plates," Z. Angew. Math. Phys. 49 (5), 786-804 (1998).

Plohr, B., D. Marchesin, P. Bedrikovetsky, and P. Krause, "Modeling hysteresis in porous media flow via relaxation," Computational Geosciences 5, 225-256 (2001)

Provatas, A., "Energetic Polymers and Plasticisers for Explosive Formulations: A Review of Recent Advances," Defence Science and Technology Organisation report DSTOTR-0966, Australia (2000).

Renlund, A., J. C. Miller, W. M. Trott, K. L. Erikson, M. L. Hobbs, R. G. Schmitt, G. W. Wellman, and M. R. Bare, "Characterization of Thermally Degraded Energetic Materials," $11^{\text {th }}$ International Detonation Symposium, Snowmass, Colorado, p. 127, 1998.

Rivera, T., "Study to Determine the Phase Diagram of the BDNPA/F System," Los Alamos National Laboratory report LA-5313 (1973).

Smilowitz, L., B. F. Henson, B. W. Asay, and P. M. Dickson, "The $\beta-\delta$ Phase Transition in the Energetic Nitramine-Octahydro-1,3,5,7-Tetranitro-1,3,5,7-Tetrazocine: Kinetics," J. Chem. Phys. 117 3789, 2002 a. 
Smilowitz, L., B. F. Henson, B. W Asay, and P. M. Dickson, "A Model for the Beta-Delta Phase Transition in PBX-9501", $12^{\text {th }}$ International Detonation Symposium, August 1116, San Diego, California, 2002b.

Smilowitz, L., B. F. Henson, B. W. Asay, P. M. Dickson, D. M. Oschwald, J. J. Romero, and G. Parker, "Morphology Changes during Thermal Decomposition of PBX9501," 13th APS Topical Conference on Shock Compression of Condensed Matter, July 20$25,2003$.

Steinberg, D. J., S. G. Cochran, and M. W. Guinan, "A Constitutive Model for Metals Applicable at High-Strain Rate," J. Appl. Phys. 51, 1498-1504 (1980).

Steinberg, D. J. and C. M. Lund, “A Constitutive Model for Strain Rates from $10^{-4}$ to $10^{6} s^{-1}, "$ J. Appl. Phys. 65, 1528-1533 (1989).

Steinberg, D. J., "Equation of State and Strength Properties of Selected Materials (revised)," Lawrence Livermore National Laboratory report UCRL-MA-106439, change 1 (February 1996).

Swegle, J. W. and A. C. Robinson, "Acceleration Instability in Elastic-Plastic Solids. I. Numerical Simulations of Plate Acceleration,” J. Appl. Phys. 66, 2838 (1989).

Tappan, A. S., A. M. Renlund, J. C. Miller, J. E. Casstevens, and M. S. Oliver, "RealTime Ultrasonic Characterization of Thermally Damaged Energetic Materials Approaching Cookoff," JANNAF Propulsion System Hazards Subcommittee, Destin, Florida, April 8-12, 2002.

Tarver, C. M. and T. D. Tran, "Thermal Decomposition Models for HMX-Based Plastic Bonded Explosives," Combustion and Flame, 137, 50-62 (2004).

Terrones, G., "Fastest-Growing Linear Rayleigh-Taylor Modes at Solid/Fluid and Solid/Solid Interfaces," Phys. Rev. E, 71 (36306), 036306-1-036306-11 (2005).

Thompson, D. G., D. J. Idar, G. T. Gray Ill, W. R. Blumenthal, C. M. Cady, E. L. Roemer, W. J. Wright, and P. D. Peterson "Quasi-Static and Dynamic Mechanical Properties of New and Virtually-aged PBX-9501 Composites as a Function of Temperature and Strain Rate," Los Alamos National Laboratory report LA-UR-02-4988 (2002).

Thynell, S. T., P. E. Gongwer, and T. B. Brill, J. Propul. Power 12:933 (1996).

Wu, X. Y., K. T. Ramesh, and T. W. Wright, "The Effects of Thermal Softening and Heat Conduction on the Dynamic Growth of Voids," Int. J. Solids Struct. 40, 4461-4478 (2003).

Zerkle, D. and L. Luck, "Modeling Cookoff of PBX-9501 with Porous Flow and Contact Resistance,” Los Alamos National Laboratory report LA-UR-03-8077 (2003).

Zerkle, D., "Cookoff Modeling with Porous Flow and Contact Resistance," Thermal Response Integrated Project Team Meeting, Los Alamos National Laboratory, February, $26^{\text {th }} 2003$. 


\section{Appendix A}

X-Sieve: CMU Sieve 2.2

X-Sender: pmd@beasley.lanl.gov

Date: Fri, 3 Jun 2005 14:46:14 -0600

To: "Francisco J. Souto" <fsouto@lanl.gov>

From: Peter Dickson <dickson@lanl.gov>

Subject: Cordin 220

X-PMX-Version: 4.7.0.111621

Francisco,

Details on the camera relating to Shot 6 as follows:

The Cordin 220 is an 8-channel gated MCP/CCD recording unit. Each channel is independently controlled for gain, gate duration, and delay from primary trigger. Gain is set in the software, while gate duration and delay are uploaded to the hardware sequencer. Eight control cables go from the sequencer to the individual MCPs.

After the camera was returned to Cordin for service before the second series of annular cookoff tests, it was returned with the cables for channels 3 and 4 swapped at the sequencer output panel. As a result, delays and gate durations programmed for these two channels were swapped on all image sets. Gains were unaffected. On shot 6 , illumination was from the rear with small-angle convergence, focused on the objective lens position of both cameras, with resulting maximum defocus in the transform plane of both optical systems (i.e., uniform rear illumination in the focal plane). The delays were set as follows:

1. $40 \mu \mathrm{s}$

2. $60 \mu \mathrm{s}$

3. $80 \mu \mathrm{s}$

4. $100 \mu \mathrm{s}$

5. $120 \mu \mathrm{s}$

6. $140 \mu \mathrm{s}$

8. * $160 \mu \mathrm{s}$

[* Please note: This email was transcribed as sent by the originator. The correct number is 7.]

Note that 3 and 4 were at 80 and $100 \mu$ s respectively. In the original images, the two apparent problems were (1) frame 3 was apparently overexposed, and (2), frame 4 appeared to be earlier than the $90 \mu \mathrm{s}$ image from the Imacon. Once 3 and 4 are swapped, the explanation is apparent frame 4 is really at $80 \mu$ s (i.e., it is correctly placed before the $90 \mu \mathrm{s}$ Imacon image) and frame 3 is really at 100 
$\mu s$, and is probably overexposed because it is recording the breakup of the confinement and seeing the direct light output from the violent reaction.

You will recall that our previous explanation, based on Blaine's calculations, was that the $100 \mu \mathrm{s}$ frame 4 was somehow mistimed and that we estimated it really represented the situation at around $76 \mu \mathrm{s}$. We now know that it was actually 80 $\mu \mathrm{s}$, so not a bad estimate. Frame 3 we concluded had been overexposed due to a gate problem, something we had seen a number of times before on that camera. In fact we now conclude that the gate may have functioned properly, but that as the image is really at $100 \mu \mathrm{s}$, the excessive brightness is probably real (note the comparison with frame 5 at $120 \mu \mathrm{s}$ ).

Peter.

$--$

\section{Peter Dickson}

DX-2 High Explosive Physics, MS J585, Los Alamos National Laboratory, Los Alamos NM 87545.

Tel. (505) 665 7830, Fax. (408) 6080314

Page (505) 6647601 or 1047601.page@pager.lanl.gov 


\section{Appendix B}

Substituting $u(x, t)$ from Eq. (4.2) into (4.1), we obtain an equation for $p(x, t)$

$$
p(x, t) \frac{\partial p(x, t)}{\partial x}=\frac{V_{T} \mu}{A_{s}} \frac{d p_{T}(t)}{d t} .
$$

Equation (A.1) is essentially an ordinary differential equation (in space) for $p(x, t)$ whose solution is parametrized by the time variable $t$. Notice that since no derivatives of $p(x, t)$ with respect to $t$ appear in Eq. (A.1), it is not a partial differential equation. Since the pressure in the tank $p_{T}(t)$ is measured, the term $d p_{T}(t) / d t$ is assumed to be known (because it can be derived from these data). At the end of the sample, the pressure is atmospheric; thus,

$$
p(L, t)=p_{0} .
$$

The solution of Eq. (A.1) subjected to the boundary condition (A.2) is

$$
p(x, t)=p_{0} \sqrt{1-\frac{2 V_{T} \mu}{A_{s} k p_{0}^{2}} \frac{d p_{T}(t)}{d t}(L-x)},
$$

which can be verified by substitution into Eq. (A.1). From Eqs. (4.2) and (A.3), the Darcian velocity (area-averaged velocity) of the gas along the length of the sample can be obtained

$$
u(x, t)=-\frac{V_{T}}{A_{s} p_{0}} \frac{d p_{T}(t)}{d t}\left[1-\frac{2 V_{T} \mu}{A_{s} k p_{0}^{2}} \frac{d p_{T}(t)}{d t}(L-x)\right]^{-1 / 2} .
$$

From Eq. (A.3), an expression for the permeability of the medium can be derived by noting that at the sample entrance the pressure is the same as that in the tank, i.e., $p(0, t)=p_{T}(t) ;$ thus,

$$
p_{T}(t)=p_{0} \sqrt{1-\frac{2 V_{T} \mu L}{A_{s} k p_{0}^{2}} \frac{d p_{T}(t)}{d t}} .
$$

Equation (4.5) is obtained by solving (A.5) for $k$.

Assuming the following parameters: $p_{0}=79009.81 \mathrm{~Pa}, V_{T}=142.1 \mathrm{~cm}^{3}, \mu=2.43 \times 10^{-5}$ Pa-s, $d p_{T}(t) / d t=-65 \mathrm{~Pa} / \mathrm{s}, t_{1}=3 \mathrm{hr}, A_{s}=7.1 \mathrm{~cm}^{2}, k=2.3 \times 10^{-16} \mathrm{~m}^{2}$, a snapshot of the pressure and velocity along the length of a porous sample is shown in Fig. (B.1). 


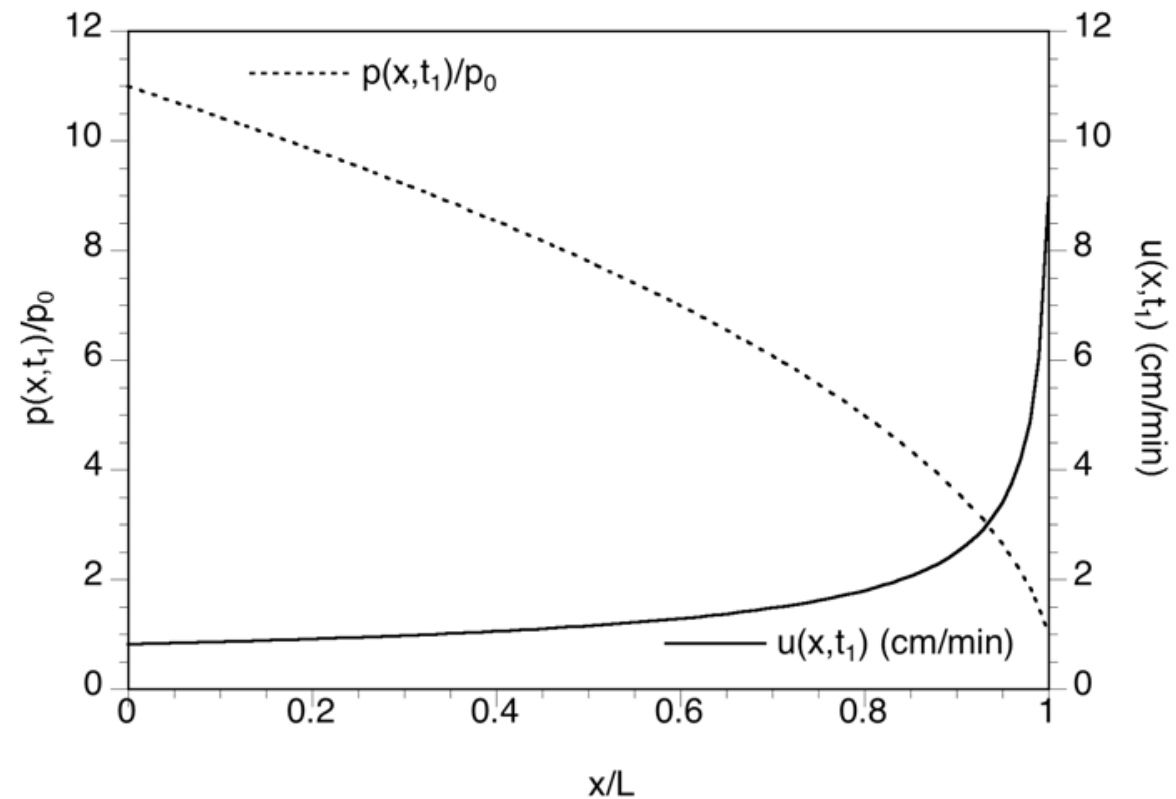

Fig. B.1. A snapshot of the pressure and velocity along the length of a porous sample. 


\section{Appendix C}

LSAC Shot $6.1 \mathrm{~km} / \mathrm{s} \mathrm{HE}, 1$ initiation point, no vent. Initiation point at $315^{\circ}$. One tracer to estimate fastest-moving point position and velocity. One tracer point for VISAR at the end cap at $330^{\circ}$, three tracers for the accelerometers, and five pins $1 \mathrm{~mm}$ from the inner wall. Twelve tracers around the outer case, 30 degrees apart, to simulate resistance wires.

\$mesh

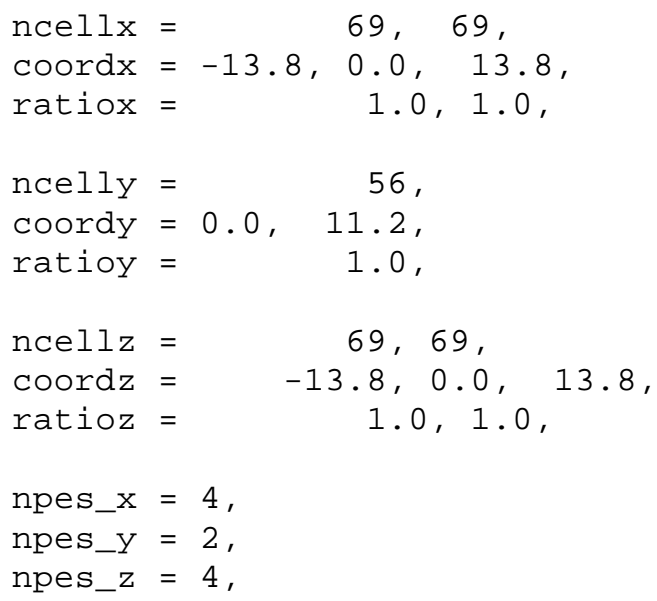




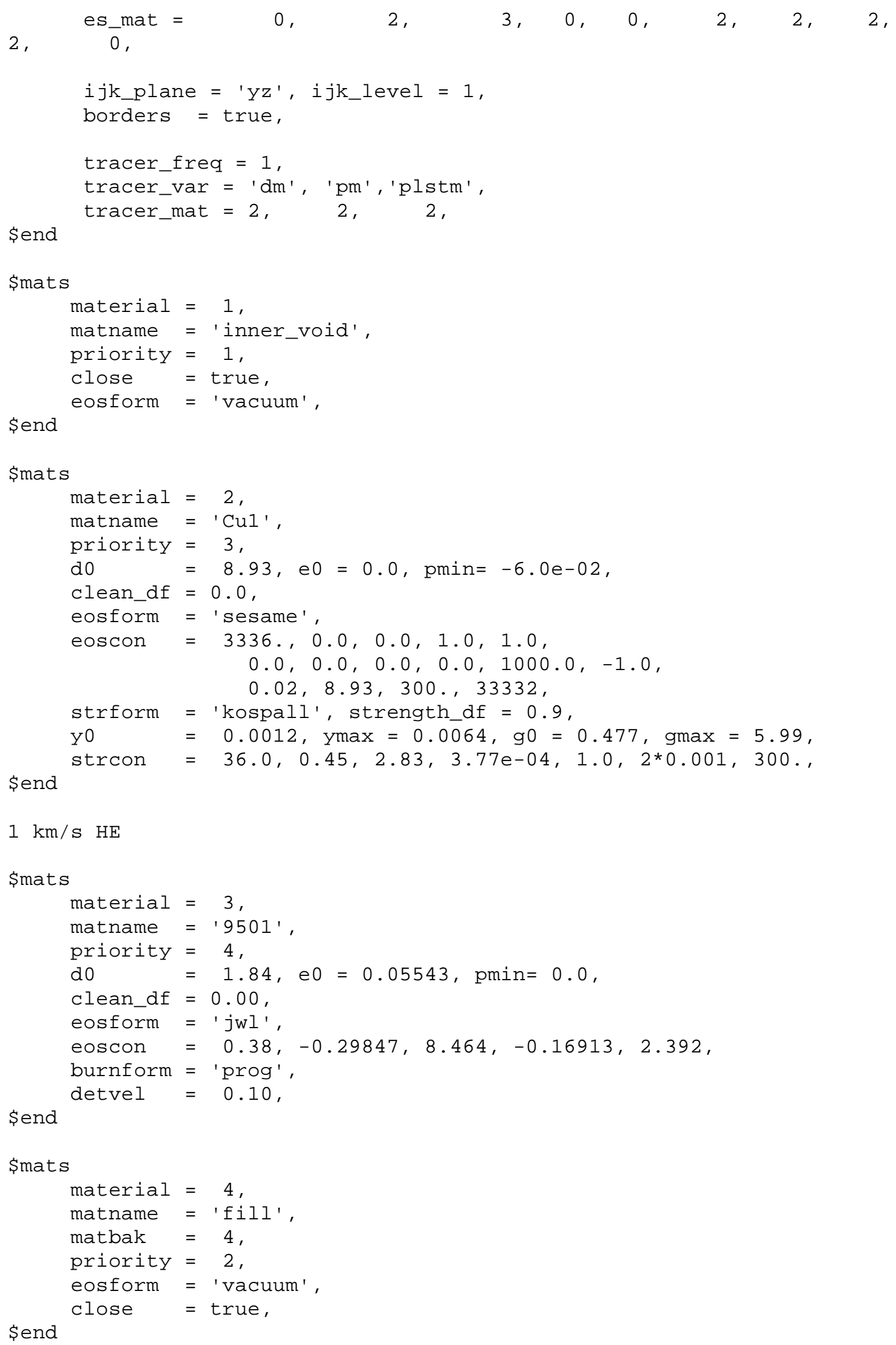




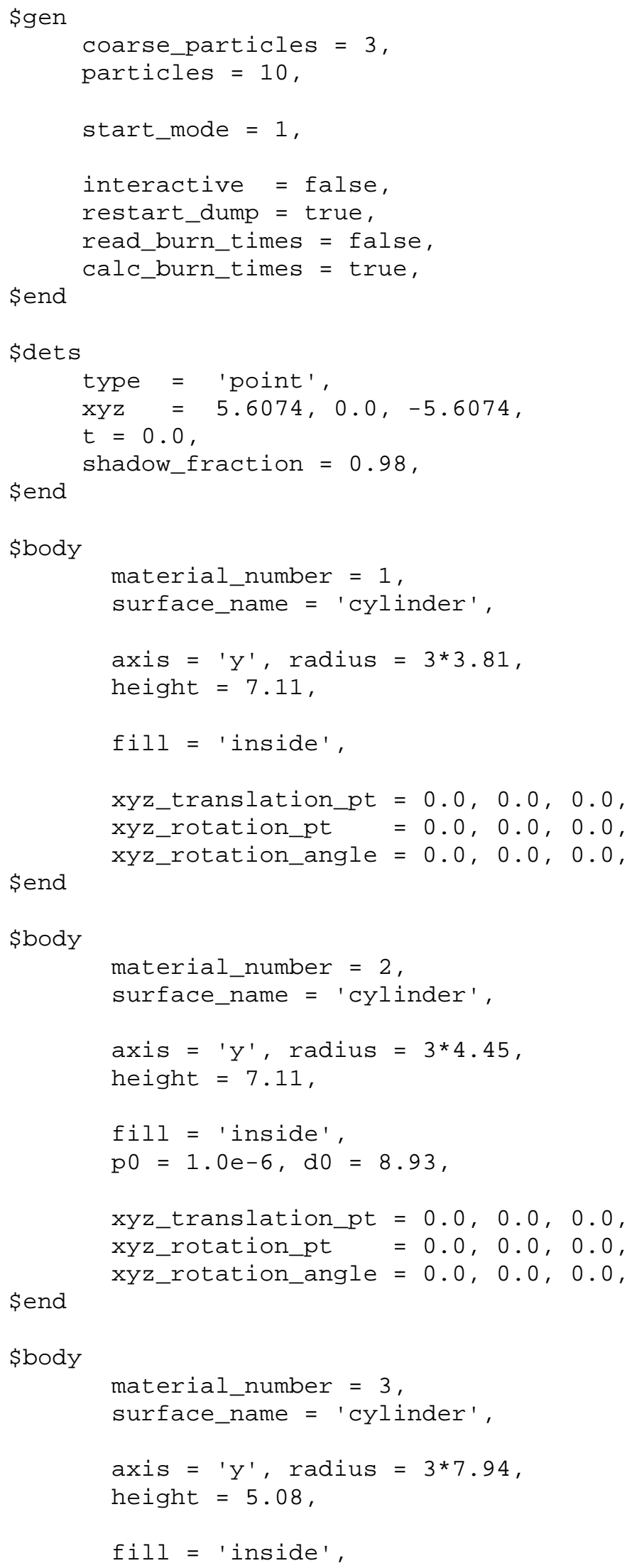




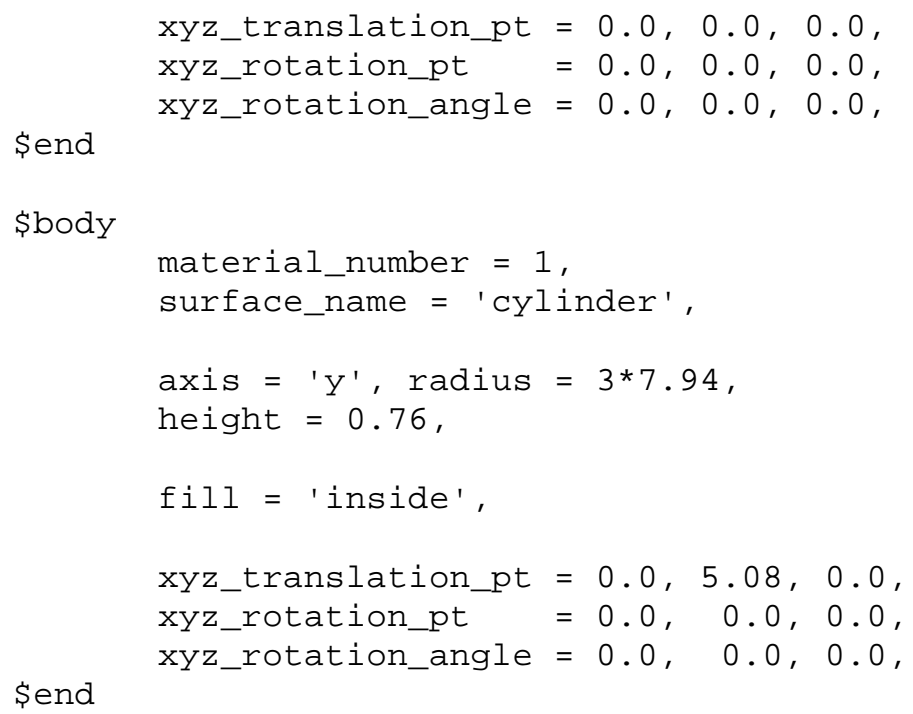




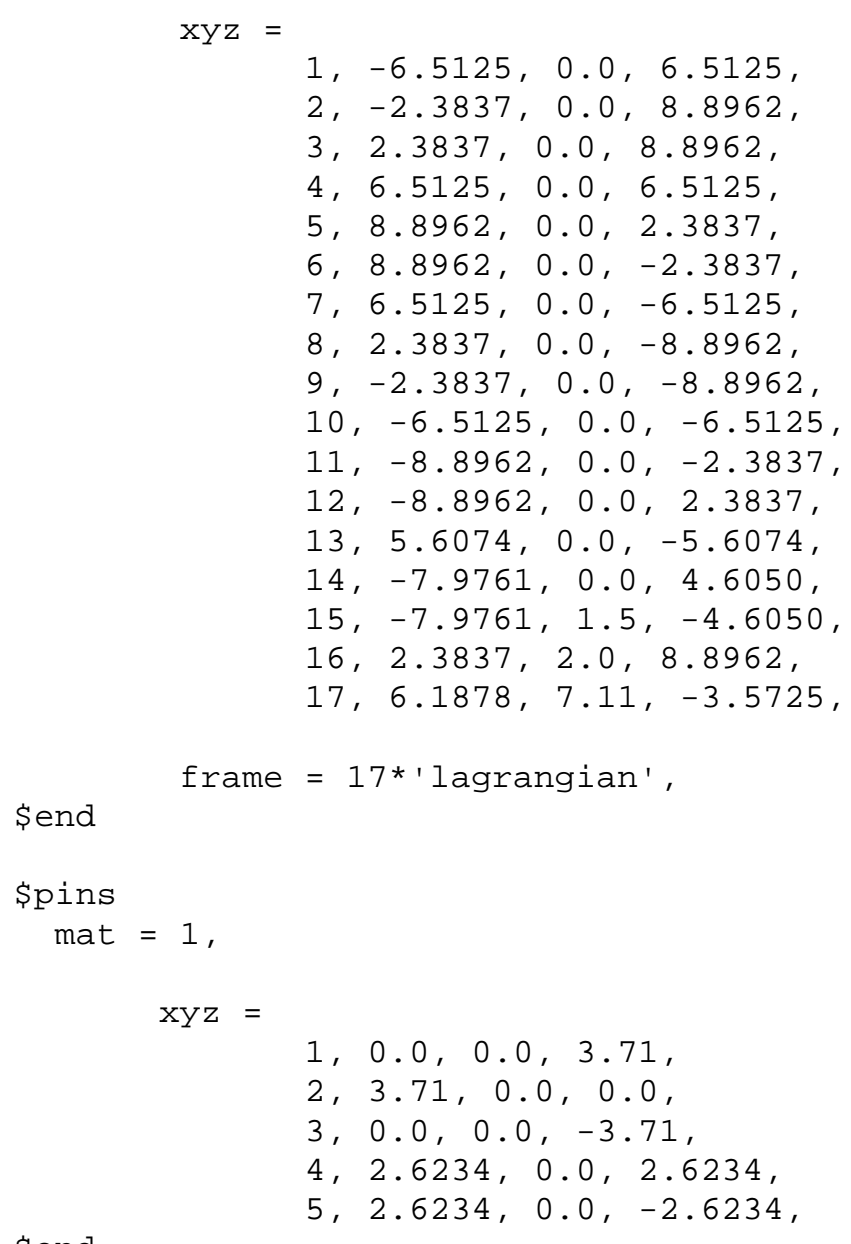


This report has been reproduced directly from the best available copy. It is available electronically on the Web (http://www.doe.gov/bridge).

Copies are available for sale to U.S. Department of Energy employees and contractors from:

Office of Scientific and Technical Information P.O. Box 62

Oak Ridge, TN 37831

(865) 576-8401

Copies are available for sale to the public from: National Technical Information Service

U.S. Department of Commerce

5285 Port Royal Road

Springfield, VA 22161

(800) 553-6847 


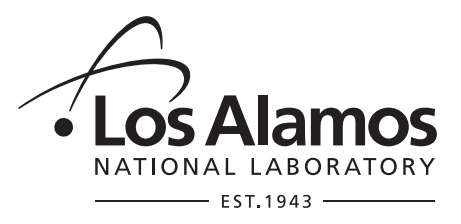

